\title{
The role of UNC-108/RAB-2 in neuronal dense core vesicle maturation in C. elegans
}

\author{
$\mathrm{PhD}$ thesis \\ In partial fulfillment of the requirements \\ for the degree "Doctor of Philosophy (PhD)" \\ in the Neuroscience graduate program \\ at the Georg August University Goettingen, \\ Faculty of Biology
}

Submitted by

Marija Sumakovic

Born in

Smederevo, Serbia

2009 


\section{Declaration}

I hereby declare that the PhD thesis entitled, "The role of UNC$108 / \mathrm{RAB}-2$ in neuronal dense core vesicle maturation in $C$. elegans", was written independently and with no other sources and aids then quoted. I would like to gratefully acknowledge Dr. Jan Hegermann and Christian Olendrowitz for the electron microscopy data, Dr. Janet Richmond for electrophysiology experiments and Dr. Steven Husson for the mass spec data.

Goettingen, $21^{\text {st }}$ September, 2009

Marija Sumakovic 


\section{Table of contents}

TABLE OF CONTENTS

ACKNOWLEDGEMENTS $\quad 5$

$\begin{array}{ll}\text { ABBREVIATION LIST } & 7\end{array}$

\begin{tabular}{l|l} 
I SUMMARY & 9
\end{tabular}

$\begin{array}{ll}\text { II INTRODUCTION } & 11\end{array}$

2.1 Intracellular trafficking is fundamental for existence of eukaryotic cells 11

2.2 Rab GTPases play a central role in organizing the membrane trafficking 12

$\begin{array}{ll}\text { 2.3 Rab dependent neuronal trafficking } & 16\end{array}$

2.4 Regulation of neurotransmission at the neuromuscular junction in C. elegans $\quad 18$

$\begin{array}{ll}2.5 \text { Regulation of neurotransmitter release } & 21\end{array}$

2.6 DCVs contribute to neurotransmission in C. elegans 25

$\begin{array}{lr}2.7 \text { DCV biogenesis } & 27\end{array}$

$\begin{array}{lr}2.8 \text { Aim of the study } & 30\end{array}$

III METHODS AND MATERIALS 32

$\begin{array}{ll}3.1 \text { Strains } & 32\end{array}$

$\begin{array}{ll}3.2 \text { Behavioral assays } & 32\end{array}$

$\begin{array}{ll}3.3 \text { Molecular biology } & 33\end{array}$

$\begin{array}{lr}3.4 \text { Crosses and transgenic strains } & 40\end{array}$

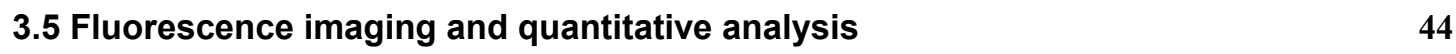

$\begin{array}{ll}\text { 3.6 Protein expression and purification } & 45\end{array}$

3.7 Antibody production and Western blotting 46

$\begin{array}{lr}3.8 \text { Cell culture } & 47\end{array}$

$\begin{array}{ll}3.9 \text { GTPase and GTP affinity assays } & 47\end{array}$

$\begin{array}{ll}3.10 \text { RNAi } & 48\end{array}$

$\begin{array}{ll}3.11 & \text { Electron microscopy }\end{array}$

$\begin{array}{lr}3.12 \text { Peptidomics } & 50\end{array}$ 
4.1 C. elegans UNC-108 is a homologue of human Rab2 and is predominantly expressed in the nervous system

4.2 unc-108/rab-2 mutants are aldicarb resistant

4.3 Mutations in UNC-108/RAB-2 affect different neurotransmitter signaling

4.4 Neuronal development is not affected in unc-108/rab-2 mutants

4.6 Increase in diacylglycerol levels rescues neurotransmission defects in unc-108 mutants to wild type level

$\begin{array}{ll}\text { 4.7 UNC-108/RAB-2 acts in DCV signaling pathway } & 77\end{array}$

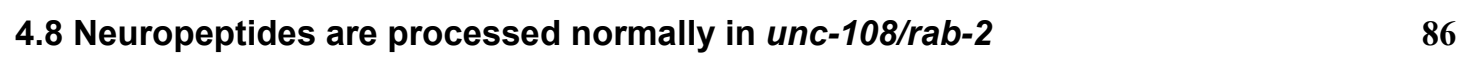

4.9 RAB-2 affects DCV signaling parallel to neuropeptides $\quad 90$

4.10 RAB-2 localizes to the Golgi, but not at the synapses 91

$\begin{array}{ll}\text { 4.11 RAB-2 mutations affect DCV maturation } & 96\end{array}$

4.12 RAB-2 mutations affect GGA localization at the Golgi/endosomal interface 105

4.13 RAB-2 cooperates with its effector RIC-19 during DCV biogenesis 106

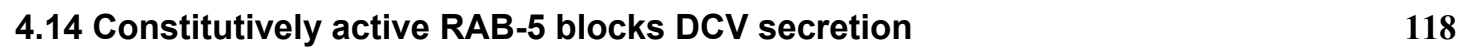

$\begin{array}{ll}\text { V DISCUSSION } & 125\end{array}$

$\begin{array}{lr}\text { 5.1 Multiple roles for RAB-2 in membrane trafficking } & 125\end{array}$

5.2 RAB-2 affects locomotion through the regulation of DCV signaling 126

$\begin{array}{ll}\text { 5.3 Molecular function of RAB-2 during DCV maturation } & 129\end{array}$

5.4 RAB-2 might affect DCV maturation through APT-9/GGA dependent sorting between Golgi and endosomes

5.5 RAB-2 cooperates with its effector RIC-19 during DCV maturation 


\section{Acknowledgements}

First, I would like to thank my supervisor Dr. Stefan Eimer for sharing his knowledge and enthusiasm for science and for support, encouragement and patience over these three years.

I am grateful to Prof. Dr. Reinhard Jahn for his great interest, comments and support for this project and for my personal development as a scientist.

I would like to thank Prof. Dr. Herbert Jäckle for his guidance and advices as a member of my PhD committee.

To the EU Neuroscience Early Stage Research Training network (NEUREST) for the financial support of this project and to the coordination team, Dr. Joachim Bormann, Eva Strehler and Alexander Zimek for their help during my studies.

I am grateful to Prof. Dr. Michael Hörner, Dr. Steffen Burkhardt and Sandra Drube for their help and support during my PhD studies.

I would like to thank Dr. Janet Richmond and Dr. Steven Husson for help with electrophysiology and mass spec experiments.

Special thanks go to my lab colleague Dr. Jan Hegermann for electron microscopy experiments.

I would like to thank all AG Eimer members, former and present, for their friendship and assistance in the lab: Nora, Ling, Christina, Katrin Schwarze, Katrin Hartwich, Jan, Caro and Sabine. To Nikhil, who kept me sane thanks to our daily lab chats. To Mandy and Christian for their enthusiasm and optimism and great support. 
Many thanks to my beloved support group Adema, Achim and Andrew and to my Geists, Katharina, Konstantina and leva for always being there for me and for all the fun we had in and out of Göttingen. Many thanks to Milena for her endless optimism and support.

I would like to thank David for keeping me sane, for his love, support and enormous patience.

Foremost, I am grateful to my family, my parents and my brother, who have stood by me, understood me and supported me every day of my life. 


\section{Abbreviation list}

BWM - body wall muscle

CAPS - Calcium dependent activator protein for secretion

CCV - clathrin coated vesicle

DAG - diacylglycerol

DCV - dense core vesicle

DGK-1 - diacylglycerol kinase

ER - endoplasmic reticulum

FLP - FMRFamide like peptides

GAP - GTPase activating proteins

GDI - GDP dissociation inhibitor

GEF - GDP/GTP exchange factor

GGA - Golgi associated, y ear containing, ADP ribosylation factor binding protein

GGT - geranylgeranyl transferase

GPCR - G protein coupled receptor

GST - Glutathion-S-transferase

HPF EM - high pressure freeze electron microscopy

HSN - hermaphrodite-specific neuron

iDCV - immature dense core vesicle

$\mathrm{IP}_{3}$ - inositol-1,4,5-triphosphate

IPTG - isopropyl $\beta$-D-thiogalactopyranoside

MALDI-TOF MS - matrix-assisted laser desorption ionization time-of-flight mass spectrometry

$\mathrm{mDCV}$ - mature dense core vesicle 
NGM - Nematode Growth Medium

NLP - neuropeptide like proteins

NMJ - neuromuscular junction

PA - phosphatidic acid

PC2 - proprotein convertase 2

$\mathrm{PI}(3,4) \mathrm{P}_{2}$ - phosphoinositol-3,4-biphosphate

PLC - phospholipase C

PMA - phorbol-12-myristate-13-acetate

REP - Rab escort proteins

RIM - Rab3-interacting protein

SV - synaptic vesicle

TFA - trifluoroacetic acid

TGN - trans Golgi network

VTC - vesicular tubular compartment 


\section{Summary}

Small GTPases of the Rab family are involved in vesicular transport between different intracellular compartments. C. elegans mutant alleles of unc-108/rab2 have been isolated based on their slow locomotion phenotype. This suggests defects in the synaptic transmission at the neuromuscular junction (NMJ) in C. elegans. In this work, I analyzed the functional defects causing reduced locomotion in unc-108/rab-2 mutant animals.

In C. elegans, fast synaptic transmission at the NMJ is mediated by release of acetylcholine from synaptic vesicles. However, for efficient neurotransmission motorneurons also rely on the dense core vesicles (DCVs) that are coreleased with SVs at the NMJ. Mutants impaired in DCV secretion have strong locomotory defects, suggesting an important role of DCV signaling in neurotransmission. We show that slow movement of unc-108/rab-2 mutants is caused by impaired DCV signaling. We further show that RAB-2 is specifically required for neuronal DCV maturation. In unc-108/rab-2 mutants, specific cargo is inappropriately lost from maturing DCVs to endosomal/lysosomal degradation system. We demonstrate that a yet unidentified DCV factor is required in addition to neuropeptides for efficient neurotransmission. Finally, we show that RIC-19, the C. elegans ortholog of the human diabetes autoantigen ICA69, is also involved in DCV maturation and recruited to Golgi membranes by activated RAB-2. Thus, we conclude that RAB-2 and its effector RIC-19 are necessary for DCV maturation and normal neurotransmission at the neuromuscular junction. 
However, RAB-2 is not the only GTPase required for efficient DCV signaling, as we could demonstrate that expression of dominant active RAB-5 is able to block DCV secretion. This suggests that particularly DCV function in neurons heavily relies on Rab dependent intracellular trafficking. 


\section{Introduction}

\subsection{Intracellular trafficking is fundamental for existence of eukaryotic cells}

An essential step in the evolution from prokaryotes to eukaryotes was the compartmentalization of the cell, which allows spatial segregation of specific cell functions. Inside the eukaryotic cells, the membranes of endoplasmic reticulum, Golgi, endosomes and other membrane enclosed compartments maintain specific differences between each organelle and the cytoplasm. Therefore, compartmentalization of a eukaryotic cell requires a highly specific transport of lipids and proteins between the organelles in order to maintain their integrity and identity (Fig. 1). Most of the transport is mediated via transport carriers, which bud off the donor compartment and fuse with an acceptor compartment, bringing cargo molecules to their destination. In the endocytotic pathway, the proteins that are internalized from the cell surface are brought to the early endosomal compartment where sorting events take place. The proteins destined to be degraded are carried to the late endosome/lysosomal compartments, while the other proteins are recycled back to the plasma membrane. In the biosynthetic pathway, proteins, once synthesized in the endoplasmic reticulum are transported through the Golgi complex and sorted from trans Golgi network to the secretory vesicles that fuse to the plasma membrane. 
Despite the heavy and dynamic membrane flow through the cell, the membrane compartments manage to maintain their structure and their biochemical identity. This demands a mechanism that insures the temporal and spatial specificity of vesicular trafficking. Rab GTPases, that belong to the family of Ras superfamily of small GTPases, have been shown to be the central regulators of vesicle budding, transport and fusion (Zerial and McBride, 2001).

\subsection{Rab GTPases play a central role in organizing the membrane trafficking}

Rab GTPases have been found in all eukaryotic cells investigated, with more than 60 different Rab family members in humans, 11 in Saccharomyces cerevisiae and 28 in Caenorhabditis elegans (Bock et al., 2001; Pereira-Leal and Seabra, 2001).Most of the Rab proteins are ubiquitously expressed, while some have a tissue specific distribution. For example, Rab3a is specifically expressed in neurons (Vonmollard et al., 1990), Rab17 in epithelial cells (Zacchi et al., 1998), Rab27a occurs predominantly in cells of hematopoietic lineage and neuroendocrine cells (Hume et al., 2001; Stinchcombe et al., 2001). Each of the Rabs is found to be localized to a specific compartment within a cell where they perform their action (Fig. 1). 


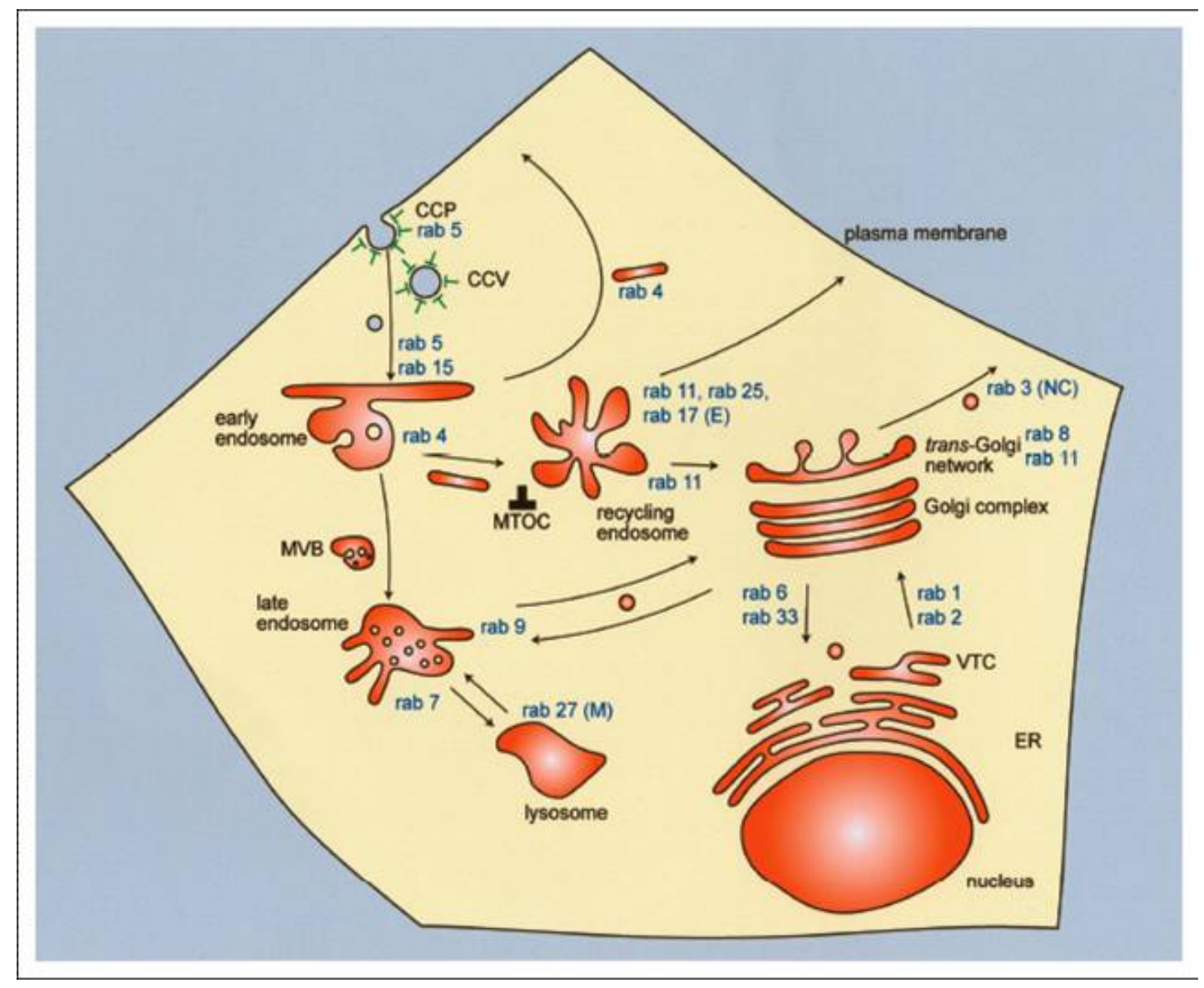

Figure 1. Intracellular localization of Rab proteins.

An overview of Rab GTPase localization in mammalian cells. CCP - clathrin coated pit, CCV - clathrin coated vesicle, M - melanosomes, E - epithelial cell type specific expression, NC neuronal cell specific, VTC - vesiculo-tubular cluster, MVB - multivesicular body, MTOC microtubule organizing center. (Adapted from (Deneka et al., 2003).).

Rab GTPases act as molecular switches cycling between an inactive GDP bound form and an active GTP bound form (Fig. 2). When in their active, GTP bound form, they are associated with the membrane and interact with downstream effector proteins (Stenmark et al., 1994b; Zerial and McBride, 2001). Rab GTPases reversibly associate with the membranes through hydrophobic geranylgeranyl groups that are attached to one or two carboxy terminal cystein residues (Anant et al., 1998; Seabra, 1996). Rab GTPases 
have similar general structure, but have different effector binding surfaces which allow recognition by wide spectra of effector proteins (Pfeffer and Aivazian, 2004). Through their effectors, Rab GTPases have been shown to regulate actin and microtubule dependent transport, vesicle budding, vesicle tethering and membrane fusion ( $\mathrm{Ng}$ and Tang, 2008). The activity of Rab GTPases is temporally and spatially controlled through the action of GDP/GTP exchange factors (GEFs) and GTPase activating proteins (GAPs) (Fukuda, 2008). The GEFs activate the Rabs by promoting the GDP exchange for GTP. The inactivation of Rabs occurs by GTP hydrolysis, which is facilitated by GAPs that enhance the intrinsic GTPase activity of the Rabs. The Rab cycle is also controlled by Rab GDP dissociation inhibitor (GDI). GDI binds to the GDP-bound form of Rab GTPases preventing the GDP dissociation and thus stabilizing the inactive form (Matsui et al., 1990). The GDIs also serve as cytosolic chaperones of geranylgeranylated Rab GTPases in the cytosol, and regulate the cycling of Rabs between membrane and the cytosol (Soldati et al., 1994; Ullrich et al., 1994; Ullrich et al., 1993). After binding of the Rab and GDI complex to the membrane, GDI displacement factor catalyses the dissociation of the complex, thereby facilitating the association of the Rab GTPases with the membrane (Sivars et al., 2003). A Similarly to GDIs, Rab escort proteins (REPs), bind to newly synthesized Rab GTPases and present them to geranylgeranyl transferases before targeting them to the membrane delivery cycle (Alexandrov et al., 1994; Seabra, 1996; Shen and Seabra, 1996). 


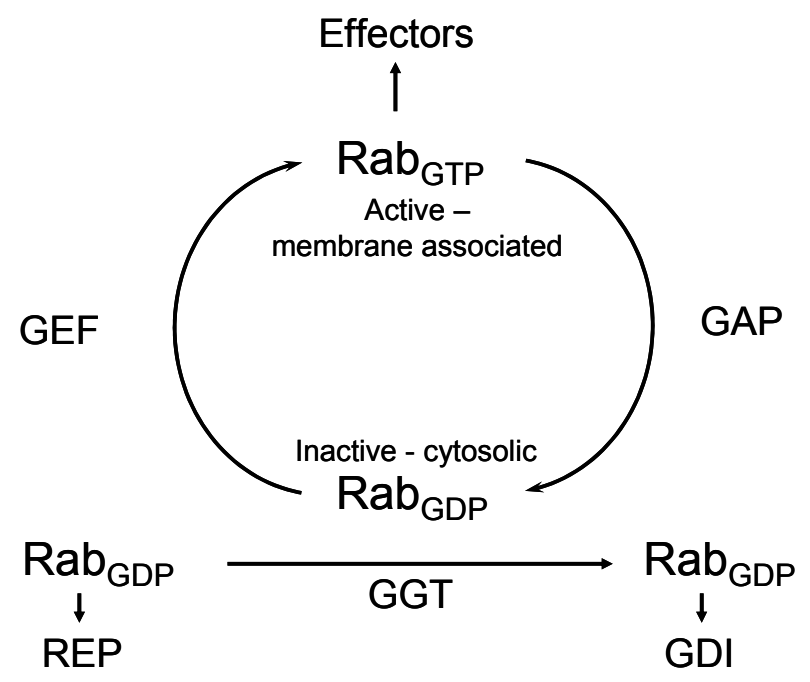

Figure 2. The Rab GTPase cycle.

The Rab GTPases act as molecular switches, cycling between GDP- and GTP- bound form. Activation of Rab protein is achieved by nucleotide exchange catalyzed by GDP/GTP exchange factor (GEF). Inactivation occurs by GTP hydrolysis, facilitated by a GTPase activating protein (GAP). The newly synthesized Rab in GDP bound form is bound to Rab escort protein (REP), which presents the Rab to geranylgeranyl transferase (GGT). GGT adds hydrophobic geranylgeranyl groups to one or two carboxyl terminal cystein residues. The geranylgeranylated Rab is then recognized by the Rab GDP dissociation inhibitor (GDI), which regulates the membrane cycle of the Rab.

In general, Rab GTPases share a common domain structure, that include the domains responsible for binding of guanine nucleotide (designated G1-G3) and phosphate/Mg2+ binding (PM1-PM3) (Fig. 12) (Valencia et al., 1991). These domains occur in all small GTPases of Ras superfamily. The Rab conserved sequences are designated as Rab family motifs (RabF) and confer five conserved short stretches (RabF1-5). The RabF motifs localize in and out of switch I and switch II regions (Pereira-Leal et al., 2001). The switch I and switch II regions change conformation significantly during the Rab cycle 
allowing the interaction with effectors and regulators (Fig. 3) (Dumas et al., 1999; Ostermeier and Brunger, 1999; Schlichting et al., 1990; Stroupe et al., 2009).
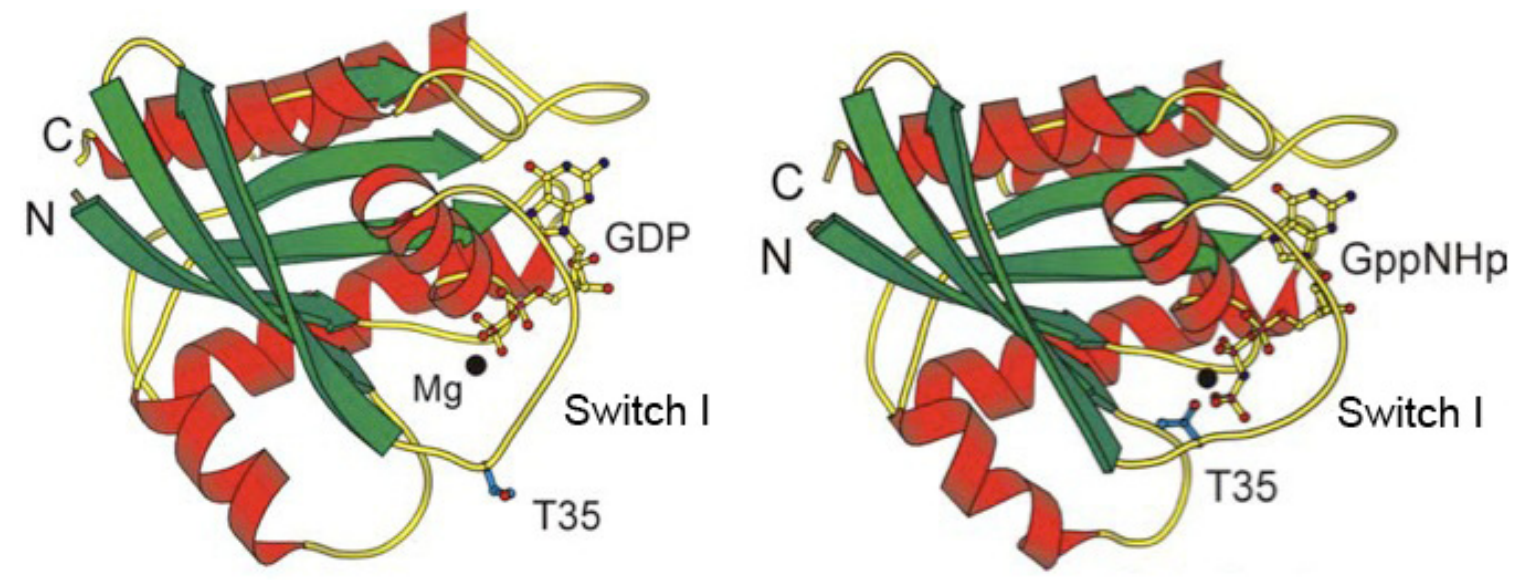

Figure 3. The conformational changes in switch I region of Rab GTPases stabilizes GTP bound active conformation.

$\mathrm{Mg}^{2+}$ stabilizes the GDP bound inactive conformation of the Rab through coordination with bound nucleotide and the GTPase. GEFs interfere with this coordination leading to dissociation of GDP from the Rab and replacement by GTP. During the activation, the switch I region of Rab GTPase, shown in yellow, changes its conformation. The stabilization of the complex is achieved by coordination between $\gamma$ phosphate of GTP and conserved residue Thr35 and prevents dissociation of GTP before hydrolysis event (Pan and Wessling-Resnick, 1998).

\subsection{Rab dependent neuronal trafficking}

Neurons are highly polarized cells with extremely specialized membrane domains, which implicates higher trafficking demands, given the transport of cargo over great distances and dynamic regulation of the cell activity. The 
membrane trafficking in neurons involves transport of proteins that are necessary for synaptic transmission and remodeling, as well as lipids necessary for neurite growth. Although neurons have the same cellular compartmentalization, the unique cellular morphology as well as the specific trafficking requirements have challenged the assumption that neurons use the same secretory machinery as the other eukaryotic cells. The role of Rab GTPases in several aspects of membrane trafficking specific for neurons has been analyzed in detail. Rab GTPases have been shown to be involved in neuronal development (Di Giovanni et al., 2006), endocytosis and retrograde transport (Deinhardt et al., 2006; Saxena et al., 2005). At the synapses, Rab GTPases perform specific functions in synaptic vesicle (SV) exocytosis and receptor recycling. An involvement of Rab3 for tethering of SVs to the presynaptic specialization and in the final stage of exocytosis has been well established (Geppert et al., 1994; Gracheva et al., 2008; Li et al., 1994; Sakane et al., 2006; Schluter et al., 2004). However, deletion of all four Rab3 isoforms in mice or mutations of the single Rab3 homologue in C. elegans only slightly affect synaptic transmission, indicating some functional redundancy among the different members of Rab family (Mahoney et al., 2006; Nonet et al., 1997; Schluter et al., 2004). Indeed it has been shown that eliminating RAB-27/AEX-6, a second synaptic Rab GTPase in C. elegans, enhances the neurotransmission defects of rab-3 mutant animals (Mahoney et al., 2006; $\mathrm{Ng}$ and Tang, 2008), suggesting that RAB-27 acts in parallel with RAB-3. Interestingly, a recent proteomic analysis of highly purified SVs revealed that, in addition to Rab3 and Rab27, a large set of other Rab GTPases can be copurified with SVs (Takamori et al., 2006). This suggests 
that multiple Rab GTPases might be needed for correct SV trafficking and regulated release. Although some of these Rabs have been functionally characterized in non-neuronal cells, their specific roles in neuronal membrane trafficking remain to be determined.

\subsection{Regulation of neurotransmission at the neuromuscular junction in C. elegans}

C. elegans has proven as a suitable organism for studying the function of novel proteins. Many of the synaptic proteins and their significance in synaptic transmission were first described in C. elegans (Ahmed et al., 1992; Avery et al., 1993; Maruyama and Brenner, 1991; Sassa et al., 1996).

C. elegans has 302 neurons for which are invariant in their location and synaptic connectivity. Their connectivity has been reconstructed by electron microscopy (White and Rainbow, 1986). The neuromuscular junction of $C$. elegans is a well studied and a well described model for studying the synaptic transmission and neuronal function. Given the simplicity of neuronal connectivity, the availability of genetic approaches and clear phenotype function relationships, $C$. elegans represents a perfect model system for studying new protein functions. 
A

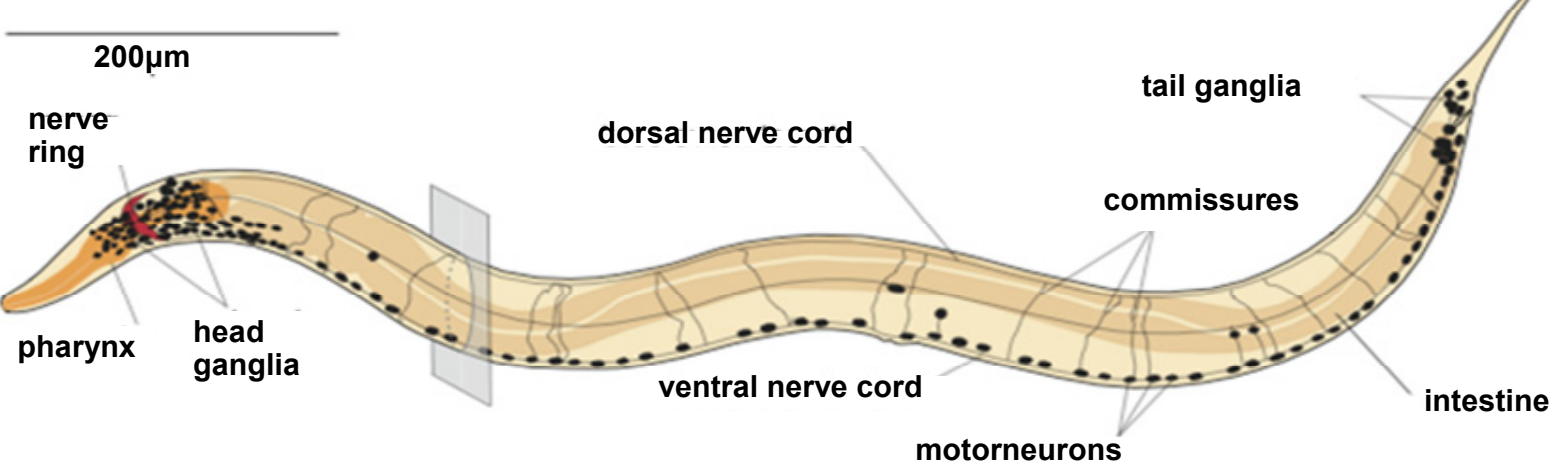

\section{B}

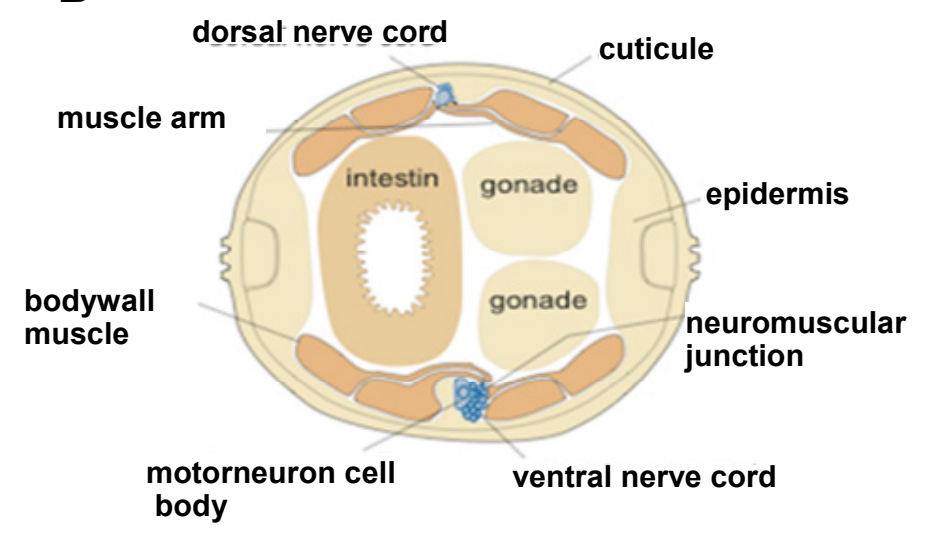

Figure 4. Organization of the motorneurons in C. elegans.

(A) Motorneurons cell bodies are located in the ventral nerve cord. The motorneurons send processes to the dorsal side of the body forming the ventral nerve cord, which stretches a long, the body and forms synapses with the body wall muscle.

(B) The body wall muscles run as quadrants along the dorsal and the ventral side of the worm. The muscles send processes, muscle arms towards the cords, forming neuromuscular junction.

The body wall muscles responsible for locomotion in C. elegans are organized in four muscle strands that run anterior-posteriorly along the entire length of the worm (Fig. 4B). Body wall muscles send projections to the nerve cords forming "en passant" synapses with motorneurons. The contraction and relaxation of body wall muscle is controlled by two neurotransmitter systems, acetylcholine and GABA (Fig. 5A). Cell bodies of cholinergic and GABAergic 


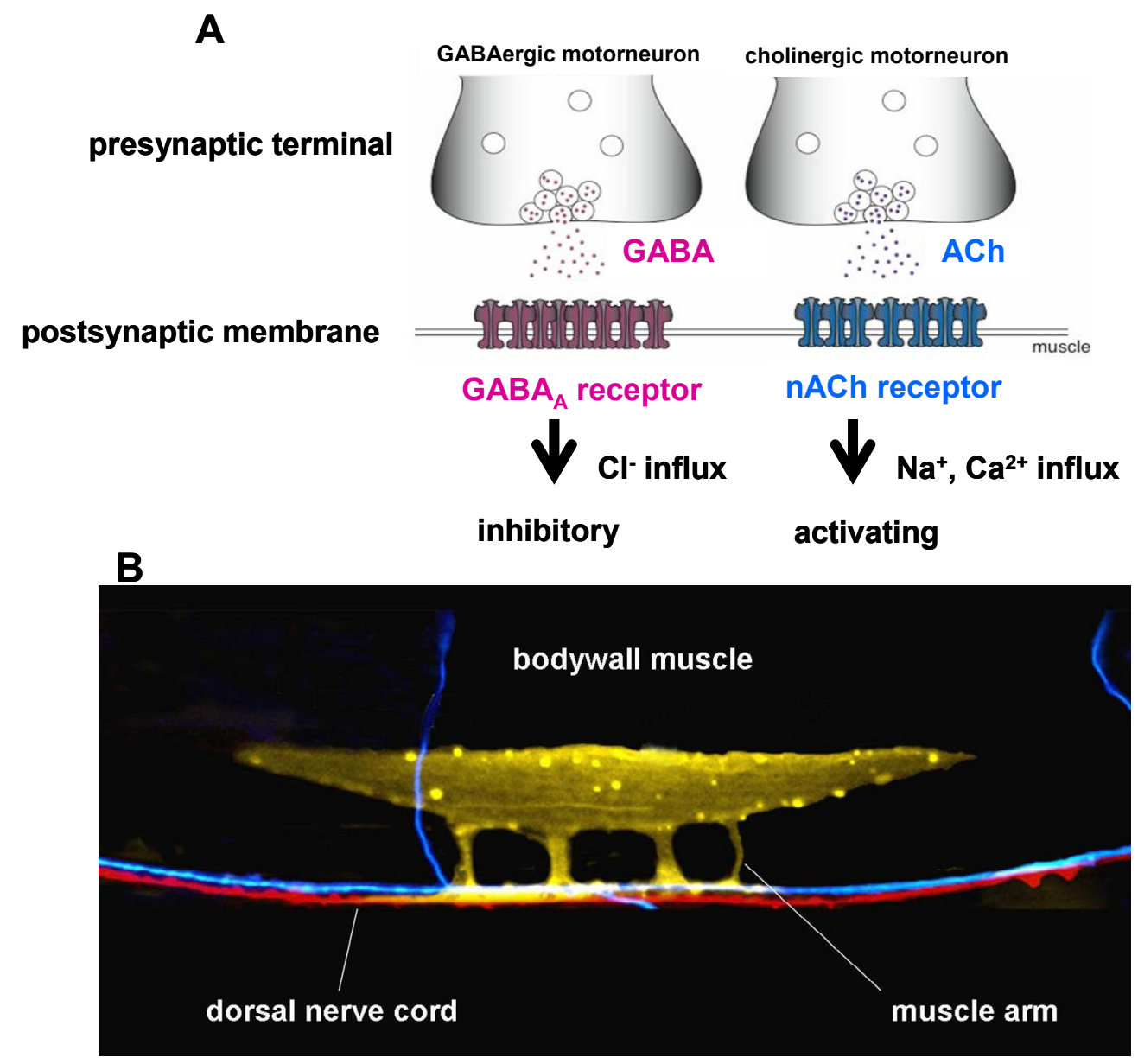

Figure 5. Locomotion in $C$. elegans is regulated by cholinergic and GABAergic systems.

(A) Body wall muscles that are responsible for locomotion in C. elegans have dual innervations, cholinergic and GABAergic. GABA synapses lead to chloride ion influx at the postsynaptic side and are inhibitory, leading to relaxation of the body wall muscle. The cholinergic receptors at the muscles are selective for sodium and calcium ions and are excitatory, leading to contraction of the body wall muscle.

(B) Cell bodies of motorneurons are located at the ventral nerve cord. The cholinergic (shown in red) and GABAergic (shown in blue) motorneurons send axons to the dorsal side of the worm body into the dorsal nerve cord, forming en passant synapses with muscle arms of body wall muscles. (Picture by Scott J. Dixon and Peter J. Roy).

motorneurons are located in the ventral nerve cord (Fig. 4) wherefrom they 
send axonal projections to the dorsal nerve cord (Fig. 4 and 5C).

C. elegans moves while lying on the side in a sine wave. The sine wave locomotion is achieved by alternate contraction and relaxation of body wall muscles. Cholinergic motorneurons activate the body wall muscles on one side of the body by releasing acetylcholine. In addition, acetylcholine simultaneously activates inhibitory GABAergic motorneurons that project their axons to the body wall muscles on the opposing side, leading to their relaxation (Walrond and Stretton, 1985a; Walrond and Stretton, 1985b). Thus, by alternating the contraction and relaxation of opposing body wall muscles, the sine wave locomotion is achieved. Thus, the locomotion in $C$. elegans largely depends on cholinergic transmission.

\subsection{Regulation of neurotransmitter release}

In motorneurons acetylcholine is packaged and released from SVs (Fig. 6). SVs dock at the active zones where they then subsequently undergo a priming step, an event which prepares the SV to respond to the calcium influx through voltage gated calcium channels that are activated by depolarization of the neuron by an arriving action potential. Upon calcium influx, SVs fuse to the plasma membrane and release their neurotransmitter into the synaptic cleft. 


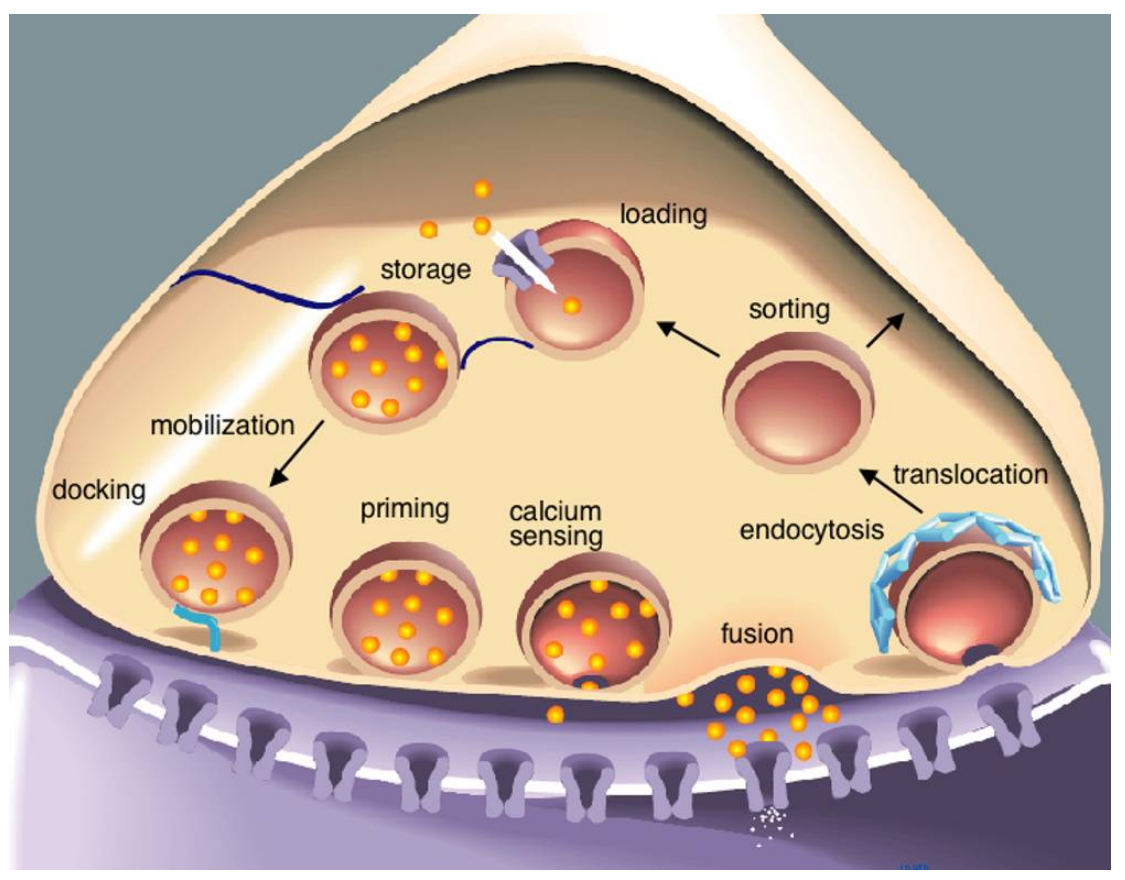

Figure 6. SV cycle.

SVs are docked to the active zone of the presynaptic terminal. Docked vesicles undergo a priming step that prepares them for the calcium influx and fusion with the plasma membrane. Upon the fusion, SVs are retrieved via endocytosis and recycled back into the presynaptic terminal. (Adapted from Richmond, 2007, wormbook, chapter: Neurotransmission)

During priming, a trimeric SNARE complex is formed between the integral SV protein synaptobrevin, the integral plasma membrane protein syntaxin and associated SNAP-25 (Fig. 7). In order for the SNARE complex to form, syntaxin transition from closed to open state has to occur. The stabilization of open conformation of syntaxin is achieved by its binding to a presynaptic protein, UNC-13 (Brose et al., 2000). Consistent with this, mutants lacking UNC-13 have loss in evoked synaptic transmission, where SVs are docked to the active zones, but fail to fuse and release the neurotransmitter efficiently (Aravamudan et al., 1999; Augustin et al., 1999; Richmond et al., 1999; Richmond et al., 2001). Furthermore, the Rab3 GTPase that is present at the 
SV interacts with Rab3-interacting protein (RIM) to activate the UNC-13 binding to syntaxin, thus promoting the SNARE complex assembly (Dulubova et al., 2005; Schoch et al., 2002; Weimer et al., 2006). Moreover, RIM has been shown to interact with calcium channels (Kiyonaka et al., 2007). Thus, this interaction positions SV in the active zone ideally for efficient response to calcium influx. $\mathrm{Ca}^{2+}$ then binds to $\mathrm{Ca}^{2+}$ sensor, synaptotagmin I, which interacts with SNARE complex driving the membrane fusion and neurotransmitter release (Koh and Bellen, 2003).

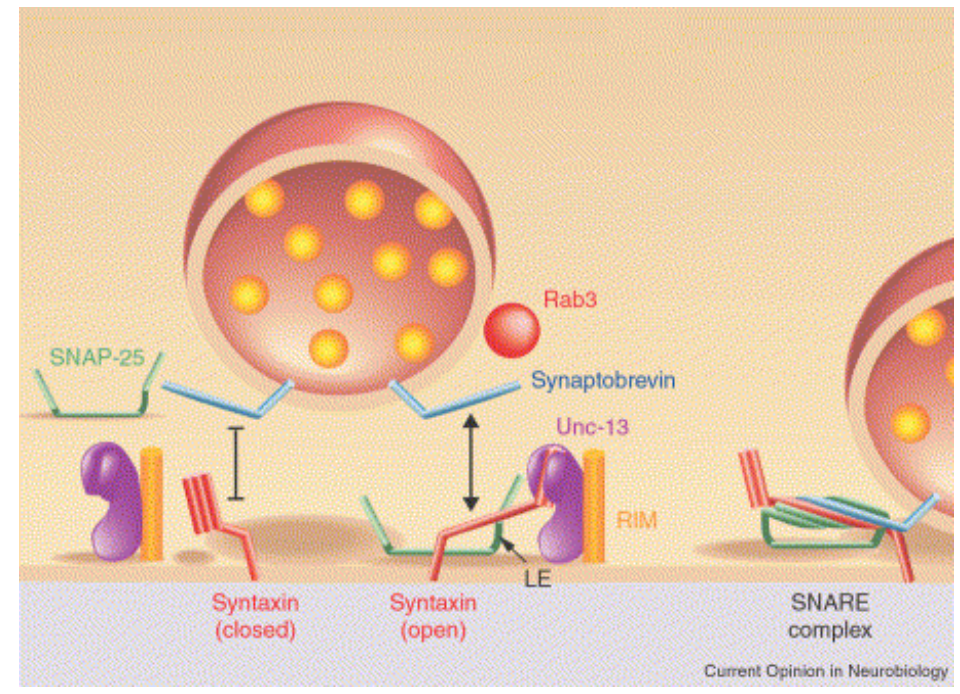

Figure 7. SV priming.

In this closed conformation, syntaxin is unavailable for SNARE complex formation, a prerequisite for vesicle priming and fusion competence. UNC-13 stabilizes the open conformation of syntaxin, driving the priming of the SV. RAB-3 GTPases that is present at the SV interacts with Rab3 interacting protein (RIM) to activate the UNC-13 binding to syntaxin. Once the SNARE complex is form, the SV is competent for fusion. (Richmond and Broadie, 2002).

The primed vesicles are termed as readily releasable pool (Lonart and Sudhof, 2000). The size of the readily releasable pool can be regulated by 
external stimuli, through a GPCR signal transduction cascade that ultimately leads to changes in diacylglycerol (DAG) levels. In C. elegans, the activation of GPCR receptors can elicit either excitatory a subunit of G-protein (EGL-30 Gaq) pathway or inhibitory a subunit (GOA-1 Gao) pathway (Fig. 8). EGL-30 activates phospholipase $C \beta$ which catalyses the cleavage of phosphoinositol3, 4-biphosphate $\left(\mathrm{PI}(3,4) \mathrm{P}_{2}\right)$ into $\mathrm{DAG}$ and inositol-1,4,5-triphosphate (IP3) (Lackner et al., 1999). The mechanism of GOA-1 action is not well understood: GOA-1 can either directly decrease the DAG levels or activate diacylglycerol kinase (DGK-1) that converts DAG to phosphatidic acid (PA) (Miller et al., 1999).

Phorbol esters, the orthologues of DAG increase the size of readily releasable pool (Searl and Silinsky, 2003; Silinsky and Searl, 2003; Waters and Smith, 2000). Phorbol esters and DAG bind to C1 domain containing proteins, UNC13 and protein kinase C (Fig. 8). Thus, phorbol esters and DAG act through SV and dense core vesicle (DCV) priming (Gillis et al., 1996; Lonart and Sudhof, 2000; Misonou et al., 1998; Sieburth et al., 2007; Stevens and Sullivan, 1998). However the exact mechanisms through which the phorbol esters lead to enhanced synaptic transmission is matter of debate. 


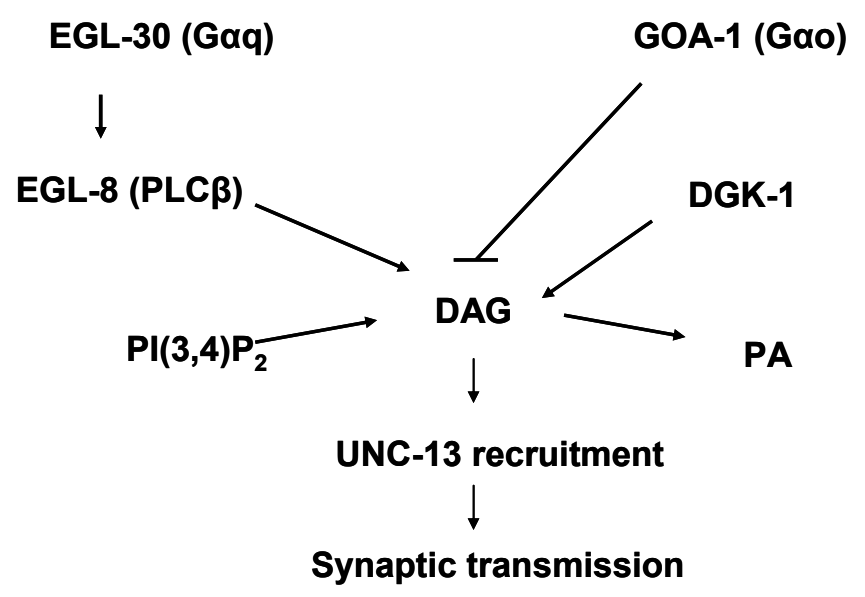

Figure 8. A model of regulation of neuromuscular junction.

The seven transmembrane receptors elicit a signal cascade at the nerve terminal either via excitatory a subunit of G-protein (EGL-30 Gaq) or inhibitory a subunit (GOA-1 Gao). The excitatory pathway leads to activation of phospholipase C (EGL-8) which converts the phosphoinositol-3,4-biphosphate $\left(\mathrm{PI}(3,4) \mathrm{P}_{2}\right)$ into diacylglycerol (DAG). DAG binds to the $\mathrm{C} 1$ domain of UNC-13, and by driving the priming of SVs increases the synaptic transmission. The inhibitory pathway acts through GOA-1 and diacylglycerol kinase (DGK-1) to convert the DAG to phosphatidic acid (PA) and therefore lower the DAG levels at the synaptic terminal.

\subsection{DCVs contribute to neurotransmission in C. elegans}

Besides the GPCR signal transduction cascade, DCV signaling also modulates the synaptic transmission. DCVs are coreleased with SVs from motorneuron axons. In C. elegans it has been clearly established that mutants with impaired release of DCVs from motorneurons exhibit severe locomotory defects (Gracheva et al., 2007a; Gracheva et al., 2007b; Sieburth et al., 2007; Zhou et al., 2007). This strongly suggests that DCV signaling largely contributes to neurotransmission. Despite its importance for neuronal activity, 
little is known about the precise function of DCVs and how their release and biogenesis are regulated.

Furthermore, the formation, trafficking and secretion of SVs and DCVs are different. While exocytosis of SVs is responsible for fast synaptic transmission, exocytosis of DCVs is slow and responsible for modulation of neurotransmission. DCVs have bigger diameter compared to SVs and appear dark on the electron micrographs due to their high protein content. They are scattered throughout the synapse and do not undergo endocytosis and recycling step, though it has been shown that each vesicle can release they cargo in stepwise manner, by repeated exocytosis (Perrais et al., 2004). Also, the higher frequency stimulation or more intense activity is required to evoke the DCV fusion compared to SVs (Bruns and Jahn, 1995).

Little is known on differential regulation of SV and DCV secretion. DCV secretion is relatively normal in the chromaffin cells isolated from Munc13 mutants (Stevens et al., 2005). However, the overexpression of Munc13 leads to increased secretion from chromaffin cells (Ashery et al., 2000). Calcium dependent activator protein for secretion (CAPS) is also involved in DCV priming. It has also been shown that CAPS is involved in DCV formation and secretion of SVs (Ann et al., 1997; Berwin et al., 1998; Renden et al., 2001). In C. elegans unc-13 and unc-31/CAPS mutants have blockage in DCV secretion and severe movement defects (Gracheva et al., 2007b; Sieburth et al., 2007), which is consistent with the proposed importance of DCV signaling at the neuromuscular junction. 


\subsection{DCV biogenesis}

DCVs are formed at the late Golgi, most likely within trans Golgi network where the proneuropeptides are packaged together with the enzymes responsible for their processing, furin type of proprotein convertases, carboxypeptidases, amidation enzymes and SNAREs (Husson et al., 2007b). The processing of proneuropeptides occurs early in the DCV biogenesis, and the formed neuropeptides are concentrated and aggregated in the DCV. This process requires that the processing enzymes are sorted together with the proneuropeptides into the DCV, as well as that the DCV is properly acidified, in order for processing enzymes to be active (Kim et al., 2006; Morvan and Tooze, 2008a). Only the fully processed neuropeptides are functional (Husson and Schoofs, 2007). Neuropeptide precursors, processing enzymes, and accessory proteins needed for DCV exocytosis have to be sorted into transport vesicles that subsequently bud off the Golgi apparatus. However, during the sorting and packaging process, constitutive secretory cargo, lysosomal enzymes, and other membrane proteins are co-packaged into DCV precursors, forming immature dense core vesicles (iDCV) (Fig. 9). To become fully functional this inappropriate cargo has to be removed from iDCVs by clathrin mediated remodeling in order to allow maturation into mature dense core vesicles (mDCV). This proofreading mechanism is important to ensure the generation of mDCVs that contain biologically active neuropeptides and undergo productive membrane fusion (Morvan and Tooze, 2008a; Tooze et al., 2001). In particular, after exit from the Golgi apparatus iDCVs have been reported to undergo syntaxin 6 and synaptotagmin IV dependent homotypic 
fusions in order to form a functionally distinct compartment from the Golgi (Morvan and Tooze, 2008b; Wendler et al., 2001). Later on, syntaxin 6 and synaptotagmin IV must be removed along with lysosomal enzymes from iDCVs since they are no longer detectable on mDCVs. The membrane remodeling of iDCVs is performed via budding of clathrin coated vesicle from maturing vesicle membrane. This provides a mechanism for proof reading the content and the membrane composition of the maturing DCV. Clathrin binding to the iDCVs is mediated by clathrin adaptor complex AP-1. The recruitment of AP-1 to maturing DCVs is ARF1 dependent. Furthermore, synaptotagmin IV is also able to bind to AP-1. In addition GGAs (Golgi associated, $y$ ear containing, ADP ribosylation factor binding protein), clathrin adaptor proteins have also been shown to be involved in clathrin mediated remodeling of iDCV membrane (Kakhlon et al., 2006). Moreover, it has been shown that GGA proteins can bind directly to ubiquitin and thus regulate the protein sorting to the endosomes (Scott et al., 2004). The cargo that is removed from iDCVs by clathrin-coated vesicles like syntaxin-6, synaptotagmin IV or mannose-6-phosphate receptor, is sorted to endosomes (Dennes et al., 2005; Morvan and Tooze, 2008b; Scott et al., 2006).

However, most of our knowledge on DCV biogenesis comes from biochemical analysis of secretory granules in $\beta$-cells or chromaffin cells. Despite their importance for regulating the neuronal activity, particularly little is known about neuronal DCVs. 


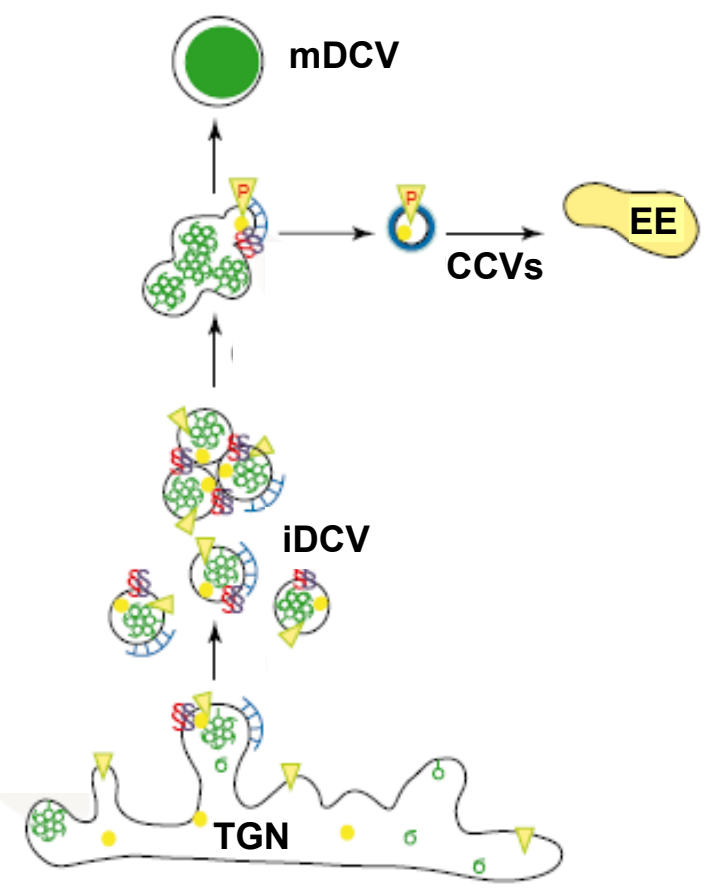

Figure 9. Biogenesis of dense core vesicles in neuroendocrine cells.

Immature dense core vesicles (iDCVs) form from the specialized region of trans Golgi network (TGN). After formation iDCVs undergo homotypic fusion forming an intermediate compartment, followed by membrane remodeling. Remodeling involves formation of clathrin coated vesicle (CCVs) which contain SNAREs, cargo and membrane proteins that are not destined for the mature dense core vesicle $(\mathrm{mDCV})$. The unnecessary proteins are than recycled in the early endosome (EE). (Adapted form Tooze et al., 2001). 


\subsection{Aim of the study}

In order to find new Rab GTPases involved in the unique membrane trafficking functions of neurons we analyzed the expression pattern of all Rab GTPases in C. elegans and focused on those which were highly enriched in the nervous system. In agreement with previous reports, we found that UNC108 , the highly conserved RAB-2 homolog in C. elegans, shows a very strong neuronal expression (Lu et al., 2008; Mangahas et al., 2008) suggesting that it might be required for neuronal function. Furthermore, unc-108/rab-2 mutants show a strong reduction in locomotion, suggesting an impairment in the neurotransmission at the neuromuscular junction. Recent studies have focused on the role of UNC-108/RAB-2 in the removal of apoptotic cells (Lu et al., 2008; Mangahas et al., 2008) and glutamate receptor trafficking (Chun et al., 2008). However, although these studies drew attention to the strong locomotory and uncoordinated phenotypes of unc-108 mutants, the precise neuronal defect that causes the impaired locomotion was not investigated.

In mammalian system, Rab2 has previously shown to be required for vesicular transport between the endoplasmic reticulum (ER) and the Golgi apparatus (Tisdale and Balch, 1996). Consistent with its localization to the pre-Golgi vesicular tubular compartment (VTC), Rab2 has been demonstrated to participate in sorting through enhancing COPI coat recruitment to the VTC (Chavrier et al., 1990; Tisdale and Balch, 1996; Tisdale and Jackson, 1998). Subsequently, active Rab2 was shown to also bind to Golgi matrix proteins and Golgi localized coil-coil proteins (Short et al., 2001; Sinka et al., 2008). 
Thus, a more general function of Rab2 for Golgi trafficking was proposed. Therefore, it is surprising that C. elegans unc-108/rab-2 mutants exhibit very specific locomotion defects more indicative of altered signaling at the neuromuscular junction.

The aim of the study was to identify the precise role of the Rab-2 in neurotransmission at the neuromuscular junction, by analyzing the neuronal trafficking in unc-108/rab-2 mutants. 


\section{Methods and materials}

\subsection{Strains}

If not otherwise stated, strains were grown at $20^{\circ} \mathrm{C}$ as described(Brenner, 1974). Strains used in this study were the wild type Bristol N2 strain, unc108(n501), unc-108(n777), ric-19(ok833), dgk-1(ok1462), rab-3(js49), unc73(e936), arls37[pmyo-3::gfp], wyls22[unc-86::gfp-rab-3], bls34[prme-8::rme8-gfp], cdls40[unc-122::gfp-cup-5], oxls12[punc-47::gfp], ccls4251;him8(e1489). unc-108(ce363) and unc-108(ce365) were kindly provided by Kenneth Miller (Oklahoma Medical Research Center, Oklahoma City, OK). KG1900 unc-108(nu415), nuls152[punc-129::gfp-snb-1], nuls168[punc129::rab-3-yfp], nuls195[punc-129::ins-22-yfp] and nuls183[punc-129::nlp-21mvenus] were provided by Joshua Kaplan (Massachusetts General Hospital, Boston, MA).

\subsection{Behavioral assays}

Movement assay. The number of body bends was assayed by visual inspection in the absence of food on Nematode Growth Medium (NGM) culture plates. A body bend corresponds to a whole $360^{\circ}$ sine wave. The number of body bands was counted over a one minute time interval. For each animal tested three consecutive intervals were averaged. For each strain 30 animals were assayed.

Drug assays. For levamisole assay, worms were grown on NGM plates containing levamisole (Sigma) in concentration gradient from $0 \mathrm{mM}$ to $1 \mathrm{mM}$ in the presence of food(Lewis et al., 1980). After one hour the number of 
paralyzed worms was counted. Worms were considered as paralyzed if they failed to move after prodding with silver wire. Aldicarb assay was performed as described (Mahoney et al., 2006). Worms were grown on plates containing $2 \mathrm{mM}$ aldicarb (Sigma Aldrich) and number of paralyzed worms was monitored over 90 min period on 10 minutes interval. For phorbol ester assay, worms were grown for 2 hours on NGM plates containing $2 \mu \mathrm{g} / \mathrm{ml}$ of phorbol-12myristate-13-acetate (PMA) (Sigma) in the presence of food (Lackner et al.,1999). After that, the animals were transferred to the plates containing $2 \mathrm{mM}$ aldicarb and $2 \mu \mathrm{g} / \mathrm{ml}$ PMA and analyzed as before. For all assays the animals were analyzed in triplets of ten worms for each strain.

Expulsion failure was monitored as described (Thomas, 1990). Total number of eggs laid and the number of unlaid eggs were quantified as described (Patel et al., 2006).

\subsection{Molecular biology}

Table 1 contains list of primers used to amplify the genes used in the study. The PCR reaction was done using Pfu polymerase (Stratagene). All genes were amplified using cDNA library as template, unless otherwise stated. All plasmids were confirmed by sequencing. pegl-30::egl-30(gf) and punc13::myr-unc-13-gfp were gift from Joshua Kaplan (Massachusetts General Hospital, Boston, MA). pHU004 and pHD84 were previously described in Marza et al., 2008. pmyo-3::GFP-Cb-5, pmyo-3::MANSII-YFP, prab3::2xFYVE-GFP and prab-3::GFP-ECOP were previously described by Eimer et al., 2007. 
Table 1. List of primers used in this study

\begin{tabular}{|c|c|c|}
\hline & Description & Primer sequence 5'to 3'end \\
\hline oGQ224 & $\begin{array}{l}\text { forward primer for amplifying unc- } \\
108 / \text { rab-2 promoter region with } \\
\text { Pstl site }\end{array}$ & $\begin{array}{l}\text { ccc ccc ctg cag tgt ggc cca agt tat } \\
\text { acc caa }\end{array}$ \\
\hline oGQ225 & $\begin{array}{l}\text { reverse primer for amplifying unc- } \\
108 / \text { rab-2 promoter region with } \\
\text { Kpnl and Agel sites }\end{array}$ & $\begin{array}{l}\text { ccc acc ggt acc tct tct gga agt tga } \\
\text { tta att gat }\end{array}$ \\
\hline oGQ023 & $\begin{array}{l}\text { forward primer for rab-2 with } \mathrm{Kpnl} \text {, } \\
\text { Agel and Ndel }\end{array}$ & $\begin{array}{l}\text { g ggg gta ccg gta cat atg tct tat gcc } \\
\text { tac ctt ttc aag tca }\end{array}$ \\
\hline oGQ024 & $\begin{array}{l}\text { reverse primer for rab-2 with } \mathrm{Xhol} \\
\text { and } B a m H I \text { sites }\end{array}$ & $\begin{array}{l}\text { gg ggg atc ctc gag tta aca gca tcc } \\
\text { aga tcc acc }\end{array}$ \\
\hline oGQ150 & $\begin{array}{l}\text { reverse primer from STOP codon } \\
\text { of golgin } 45 \text { with } X \text { hol restriction } \\
\text { site }\end{array}$ & $\begin{array}{l}\text { gaa ttc tcg agt taa aga agt tga ata } \\
\text { tct cga ccc }\end{array}$ \\
\hline oGQ152 & $\begin{array}{l}\text { forward primer from start codon of } \\
\text { golgin } 45 \text { with } N \text { col restriction site }\end{array}$ & gga tcc atg gaa ccg gtg act tcg t \\
\hline oGQ101 & $\begin{array}{l}\text { forward mutagenesis primer for } \mathrm{Ce} \\
\text { rab-2 that introduces the GDP } \\
\text { restricting mutation S2ON }\end{array}$ & $\begin{array}{l}\text { gta gga aaa aac tgc ttg ctc ctt cag } \\
\text { ttt acc }\end{array}$ \\
\hline oGQ102 & $\begin{array}{l}\text { reverse mutagenesis primer for } \mathrm{Ce} \\
\text { rab-2 that introduces the GDP } \\
\text { restricting mutation S2ON }\end{array}$ & $\begin{array}{l}\text { gag caa gca gtt ttt tcc tac tcc agt } \\
\text { atc ccc }\end{array}$ \\
\hline oGQ107 & $\begin{array}{l}\text { forward mutagenesis primer for } \mathrm{Ce} \\
\text { rab-2 that introduces the GTP } \\
\text { restricting mutation Q65L }\end{array}$ & $\begin{array}{l}\text { aca gcc gga ctg gaa tca ttc cgc tcc } \\
\text { atc act }\end{array}$ \\
\hline oGQ108 & $\begin{array}{l}\text { reverse mutagenesis primer for } \mathrm{Ce} \\
\text { rab-2 that introduces the GTP } \\
\text { restricting mutation Q65L }\end{array}$ & $\begin{array}{l}\text { gaa tga ttc cag tcc ggc tgt gtc cca } \\
\text { aat ttg }\end{array}$ \\
\hline oGQ109 & $\begin{array}{l}\text { forward mutagenesis primer for } \mathrm{Ce} \\
\text { rab-1 that introduces the GTP } \\
\text { restricting mutation Q70L }\end{array}$ & $\begin{array}{l}\text { act gct ggt ctg gaa cgt ttc cgc aca } \\
\text { att aca }\end{array}$ \\
\hline oGQ110 & $\begin{array}{l}\text { reverse mutagenesis primer for } \mathrm{Ce} \\
\text { rab-1 that introduces the GTP } \\
\text { restricting mutation } \mathrm{Q} 70 \mathrm{~L}\end{array}$ & $\begin{array}{l}\text { gaa acg ttc cag acc agc agt gtc } \\
\text { cca gat ttg }\end{array}$ \\
\hline oGQ21 & $\begin{array}{l}\text { forward rab-1 primer that } \\
\text { introduces a Kpnl, Notl, and a } \\
\text { Ndel site up-stream of the start } \\
\text { ATG }\end{array}$ & $\begin{array}{l}\text { ggg ggt acc ggc ggc cgc cat atg } \\
\text { gca gca atg aac cct gaa }\end{array}$ \\
\hline oGQ22 & $\begin{array}{l}\text { reverse rab-1 primer that } \\
\text { introduces a } E c o R I \text { and a BamHI } \\
\text { site after the stop codon }\end{array}$ & $\begin{array}{l}\text { gg gga tcc gaa ttc tta aca aca tcc } \\
\text { acc gct ctt }\end{array}$ \\
\hline oSE262 & $\begin{array}{l}\text { forward mutagenesis primer for } \mathrm{Ce} \\
\text { RAB-6.1 that introduces the GTP } \\
\text { restricting mutation Q70L }\end{array}$ & $\begin{array}{l}\text { acc get ggc ctg gaa cgt ttc cga tct } \\
\text { att }\end{array}$ \\
\hline oSE263 & $\begin{array}{l}\text { reverse mutagenesis primer for } \mathrm{Ce} \\
\text { rab-6.1 that introduces the GTP } \\
\text { restricting mutation } \mathrm{Q} 70 \mathrm{~L}\end{array}$ & $\begin{array}{l}\text { gaa acg ttc cag gcc agc ggt atc } \\
\text { cca caa } \\
\text { ttg }\end{array}$ \\
\hline
\end{tabular}




\begin{tabular}{|c|c|c|}
\hline oSE253 & $\begin{array}{c}\text { forward primer for rab-6.1 } \\
\text { introduces Kpnl, Agel and Nco I } \\
\text { sites }\end{array}$ & $\begin{array}{l}\text { g ggg gta ccg gta tcc atg get gat ttc } \\
\text { aca aat aac g }\end{array}$ \\
\hline oSE254 & $\begin{array}{l}\text { reverse primer for rab- } 6.1 \\
\text { that introduces a Xhol and a } \\
\text { EcoRl site after the stop } \\
\text { codon }\end{array}$ & $\begin{array}{l}\text { gg gga att ctc gag tta aca cgg aca } \\
\text { ttg } \\
\text { acg gcc }\end{array}$ \\
\hline oSE255 & $\begin{array}{l}\text { forward primer for rab-6.2 that } \\
\text { introduces a Kpnl, Agel, and a } \\
\text { Ndel site in front of the start ATG }\end{array}$ & $\begin{array}{l}\text { c ccg gta ccg gta cat atg tcg gac ttt } \\
\text { ggt aat ccg }\end{array}$ \\
\hline oSE256 & $\begin{array}{l}\text { reverse primer for rab-6.2 } \\
\text { that introduces a Xhol and a } \\
\text { EcoRl site after the stop codon }\end{array}$ & $\begin{array}{l}\text { cc cga att ctc gag tta gca cca gca } \\
\text { cga } \\
\text { tcc ctc }\end{array}$ \\
\hline oSE264 & $\begin{array}{l}\text { forward mutagenesis primer for Ce } \\
\text { rab-6.2 that introduces the GTP } \\
\text { restricting mutation Q69L }\end{array}$ & $\begin{array}{l}\text { acc gct gga ctg gaa cgt ttc cgc tca } \\
\text { ctc atc }\end{array}$ \\
\hline oSE265 & $\begin{array}{l}\text { reverse mutagenesis primer for Ce } \\
\text { rab-6.2 that introduces the GTP } \\
\text { restricting mutation Q69L }\end{array}$ & $\begin{array}{l}\text { gaa acg ttc cag tcc agc ggt gtc cca } \\
\text { aag } \\
\text { ttg }\end{array}$ \\
\hline oSE196 & $\begin{array}{l}\text { forward primer for GRASP55 that } \\
\text { introduces a Kpnl, a Agel, and a } \\
\text { Ncol site upsteam of the start ATG }\end{array}$ & $\begin{array}{l}\text { g ggg gta ccg gtcc atg gga agc agt } \\
\text { gaa agt gta }\end{array}$ \\
\hline oSE197 & $\begin{array}{l}\text { reverse primer for } \\
\text { GRASP55 that introduces Notl } \\
\text { site, a } \\
\text { stop codon and a Xhol site }\end{array}$ & $\begin{array}{l}\text { ggg ctc gag tta gcg gcc gcc gcc ctg } \\
\text { tgg tgg agc agg tgg ata }\end{array}$ \\
\hline oGQ165 & $\begin{array}{l}\text { forward mutagenesis primer for } \mathrm{Ce} \\
\text { rab- } 2 \text { that introduces the } \mathrm{D} 122 \mathrm{~N} \\
\text { mutation }\end{array}$ & $\begin{array}{l}\text { aat aag agt aat ctg gaa gcc cgt cgc } \\
\text { gaa gtg }\end{array}$ \\
\hline oGQ166 & $\begin{array}{l}\text { reverse mutagenesis primer for } \mathrm{Ce} \\
\text { rab-2 that introduces the } \mathrm{D} 122 \mathrm{~N} \\
\text { mutation }\end{array}$ & $\begin{array}{l}\text { ggc ttc cag att act ctt att tcc aat } \\
\text { caa cat }\end{array}$ \\
\hline oGQ167 & $\begin{array}{l}\text { forward mutagenesis primer for } \mathrm{Ce} \\
\text { rab-2 that introduces the S149F } \\
\text { mutation }\end{array}$ & $\begin{array}{l}\text { atg gag aca ttc gcc agg acg gct } \\
\text { gcc acc gtg }\end{array}$ \\
\hline oGQ168 & $\begin{array}{l}\text { reverse mutagenesis primer for } \mathrm{Ce} \\
\text { rab-2 that introduces the } \mathrm{S} 149 \mathrm{~F} \\
\text { mutation }\end{array}$ & $\begin{array}{l}\text { cgt ctt ggc gga tgt ctc cat gaa tac } \\
\text { gag tcc }\end{array}$ \\
\hline oGQ530 & $\begin{array}{l}\text { forward primer for } \mathrm{PH} \text { domain of } \\
\mathrm{PLC} \delta \text { mutagenesis }\end{array}$ & $\begin{array}{l}\text { ggg tct aga aaa aaa aga gag tga } \\
\text { ggt gcc }\end{array}$ \\
\hline oGQ531 & $\begin{array}{l}\text { reverse primer for } \mathrm{PH} \text { domain of } \\
\mathrm{PLC} \delta \text { mutagenesis }\end{array}$ & $\begin{array}{l}\text { ggg gaa gcg ctc tct cct ggt tga gct } \\
\text { gga cgc cac cgc cag gag ctg gct } \\
\text { gcc ctt cag }\end{array}$ \\
\hline oGQ230 & $\begin{array}{l}\text { forward primer for rab-3 with } \mathrm{Ndel} \\
\text { and } \mathrm{Ncol} \text { restriction sites }\end{array}$ & $\begin{array}{l}\text { ggg cat atg gcc atg gcg gct ggc } \\
\text { gga caa cct }\end{array}$ \\
\hline oGQ231 & $\begin{array}{l}\text { reverse for rab-3 with } \mathrm{BamHI} \text { and } \\
\text { Xhol restriction sites }\end{array}$ & $\begin{array}{l}\text { ggg gga tcc ctc gag tta gca att gca } \\
\text { ttg ctg ttg }\end{array}$ \\
\hline
\end{tabular}




\begin{tabular}{|c|c|c|}
\hline oGQ139 & $\begin{array}{l}\text { reverse } m R F P \\
\text { Cherry with introns } \\
\text { primer that removes the stop } \\
\text { codon and introduces a } \\
\text { Notl,Eco47III, and } \\
\text { a Agel site afterwards }\end{array}$ & $\begin{array}{l}\text { cgc tac cgg tcc agc get gcg gcc } \\
\text { gcc gct agc ctt ata caa ttc atc }\end{array}$ \\
\hline oGQ137 & $\begin{array}{l}\text { forward mRFP Cherry with introns } \\
\text { primer that introduces a Ncol, } \\
\text { BamHI, Kpnl, Nhel and Smal half } \\
\text { site upstream of the start codon }\end{array}$ & $\begin{array}{l}\text { ggg gct agc tcg gta ccg gga tcc atg } \\
\text { gtc tca aag ggt }\end{array}$ \\
\hline oGQ323 & $\begin{array}{l}\text { forward primer for syntaxin-6 that } \\
\text { start at the start ATG and } \\
\text { introduces a Ndel } \\
\text { and a BamHI site upstream }\end{array}$ & $\begin{array}{l}\text { gga tcc cat atg agc aac tac cga tac } \\
\text { tca }\end{array}$ \\
\hline oGQ324 & $\begin{array}{l}\text { reverse primer for syntaxin- } 6 \text { that } \\
\text { start at the stop codon and } \\
\text { introduces a EcoRI } \\
\text { site downstream }\end{array}$ & $\begin{array}{l}\text { ccc gaa ttc tca gac cag aag gac } \\
\text { aaa gac }\end{array}$ \\
\hline oGQ285 & $\begin{array}{l}\text { forward primer for } r a b-5 \text { that start } \\
\text { at the start ATG and introduces a } \\
\text { Ndel and a BamHI site upstream }\end{array}$ & $\begin{array}{l}\text { gga tcc cat atg gcc gcc cga aac } \\
\text { gca gga }\end{array}$ \\
\hline oGQ286 & $\begin{array}{l}\text { reverse primer for rab- } 5 \text { that start } \\
\text { at the stop codon and introduces a } \\
\text { EcoRI site downstream }\end{array}$ & $\begin{array}{l}\text { ccc gaa ttc tta ttt aca gca tga acc } \\
\text { ctt ttg }\end{array}$ \\
\hline oGQ283 & $\begin{array}{l}\text { forward primer for rab-7 that start } \\
\text { at the start ATG and introduces a } \\
\text { Ndel and a BamHI site upstream }\end{array}$ & $\begin{array}{l}\text { gga tcc cat atg tcg gga acc aga } \\
\text { aag aag }\end{array}$ \\
\hline oGQ284 & $\begin{array}{l}\text { reverse primer for rab- } 7 \text { that start } \\
\text { at the stop codon and introduces a } \\
\text { Xhol site downstream }\end{array}$ & $\begin{array}{l}\text { ccc ctc gag tta aca att gca tcc cga } \\
\text { att }\end{array}$ \\
\hline oGQ769 & $\begin{array}{l}\text { forward primer for } I m p-1 \text { that starts } \\
\text { at the start ATG and introduces a } \\
\text { BamHI, Kpnl and Agel site } \\
\text { upstream }\end{array}$ & $\begin{array}{l}\text { gga tcc agg gta ccg gta atg ttg aaa } \\
\text { tcg ttt gtc atc ttg }\end{array}$ \\
\hline oGQ770 & $\begin{array}{l}\text { reverse primer for Imp-1 that } \\
\text { removes the stop codon and } \\
\text { introduces a } \\
\text { Notl, Nhel and Eco47III half site } \\
\text { afterwards }\end{array}$ & $\begin{array}{l}\text { gct agc gcg gcc gcc acc gac gct } \\
\text { ggc ata tcc ttg tct c }\end{array}$ \\
\hline oGQ673 & $\begin{array}{l}\text { forward mutagenesis primer for Ce } \\
\text { rab-5 that introduces GDP } \\
\text { restriction mutation S33N }\end{array}$ & $\begin{array}{l}\text { gta ggc aaa aac tct ctc gta ttg cga } \\
\text { ttc gtc }\end{array}$ \\
\hline oGQ674 & $\begin{array}{l}\text { reverse mutagenesis primer for } \mathrm{Ce} \\
\text { rab- } 5 \text { that introduces GDP } \\
\text { restriction mutation S33N }\end{array}$ & $\begin{array}{l}\text { tac gag aga gtt ttt gcc tac agc act } \\
\text { ttc tcc }\end{array}$ \\
\hline oGQ675 & $\begin{array}{l}\text { forward mutagenesis primer for } \mathrm{Ce} \\
\text { rab-5 that introduces GTP }\end{array}$ & $\begin{array}{l}\text { act gca gga ctg gaa aga tat cat tca } \\
\text { ttg gca }\end{array}$ \\
\hline
\end{tabular}




\begin{tabular}{|l|l|l|}
\hline oGQ676 & $\begin{array}{l}\text { restriction mutation Q 78 L } \\
\text { reverse mutagenesis primer for Ce } \\
\text { rab-5 that introduces GTP } \\
\text { restriction mutation Q 78 L }\end{array}$ & $\begin{array}{l}\text { ata tct ttc cag tcc tgc agt atc cca } \\
\text { gat ttc }\end{array}$ \\
\hline oGQ429 & $\begin{array}{l}\text { forward primer for apt-9 GGA that } \\
\text { starts at the ATG and introduces a } \\
\text { Bglll, Agel and Ndel site upstream }\end{array}$ & $\begin{array}{l}\text { aga tct tca ccg gta cat atg gag gaa } \\
\text { gaa cca gtg cgt }\end{array}$ \\
\hline oGQ430 & $\begin{array}{l}\text { reverse primer for apt-9 GGA that } \\
\text { matches the stop codon and } \\
\text { introduces a Xhol } \\
\text { and EcoR/ site afterward }\end{array}$ & $\begin{array}{l}\text { gg gga att ctc gag tca aaa att aat } \\
\text { att aaa aat atg ttc tgg }\end{array}$ \\
\hline oGQ592 & $\begin{array}{l}\text { Forward primer for ric-19 with Kpnl } \\
\text { and Ncol restriction sites }\end{array}$ & $\begin{array}{l}\text { ggg ggt acc gcc atg gct gca cag ttt } \\
\text { tac gaa agg aac aca }\end{array}$ \\
\hline oGQ593 & $\begin{array}{l}\text { Reverse primer for ric-19 with Notl } \\
\text { site, stop codon and Xhol site }\end{array}$ & $\begin{array}{l}\text { ggg ctc gag tca gcg gcc gcc caa } \\
\text { caa gtt atc ctc att ctc tct }\end{array}$ \\
\hline
\end{tabular}

Table 2. List of constructs made and used in this study

\begin{tabular}{|l|l|}
\hline Construct & Description \\
\hline prab-2::gfp & $\begin{array}{l}\text { The fragment was amplifyed by PCR using } \\
\text { oGQ224 and oGQ225 primers and exchanged } \\
\text { for rab-3 promoter in pHU004 using Pstl and } \\
\text { Kpnl sites }\end{array}$ \\
\hline pGBKT7 RAB-2WT & $\begin{array}{l}\text { rab-2 was amplified by PCR using oGQ023 and } \\
\text { oGQ024 primers and cloned into pGBKT7 using } \\
\text { Ndel and BamHI sites }\end{array}$ \\
\hline pGADT7 Golgin45 & $\begin{array}{l}\text { golgin45 was amplified by PCR using oGQ150 } \\
\text { and oGQ152 primers and cloned into pGADT7 } \\
\text { vector using Ncol and Xhol restriction sites }\end{array}$ \\
\hline pGBKT7 RAB-2 (S20N) DN & $\begin{array}{l}\text { S20N mutation was induced via PCR directed } \\
\text { mutagenesis and the fragment was cloned into } \\
\text { pGBKT7 vector using Ndel and BamHI sites }\end{array}$ \\
\hline pGBKT7 RAB-2 (Q65L) DA & $\begin{array}{l}\text { Q65L mutation was induced via PCR directed } \\
\text { mutagenesis and the fragment was cloned into } \\
\text { pGBKT7 vector using Ndel and BamHI sites }\end{array}$ \\
\hline pGBKT7 RAB-1 (Q70L) DA & $\begin{array}{l}\text { Q70L mutation was introduced via PCR directed } \\
\text { mutagenesis using oGQ110 and oGQ109 } \\
\text { primers and oGQ021 and oGQ022 for amplifying } \\
\text { the fragment and then cloned into pGBKT7 } \\
\text { vector with Ndel and BamHI sites }\end{array}$ \\
\hline pGBKT7 RAB-6.1 (Q69L) DA & $\begin{array}{l}\text { Q70L mutation was introduced via PCR directed } \\
\text { mutagenesis using oSE262 and oSE263 primers } \\
\text { and oSE253 and oSE254 for amplifying the } \\
\text { fragment and then cloned into pGBKT7 vector } \\
\text { with Ncol and EcoR/ sites }\end{array}$ \\
\hline Q69L mutation was introduced via PCR directed \\
\hline
\end{tabular}




\begin{tabular}{|c|c|}
\hline & $\begin{array}{l}\text { mutagenesis using oSE264 and oSE265 primers } \\
\text { and oSE255 and oSE256 for amplifying the } \\
\text { fragment and then cloned into pGBKT7 vector } \\
\text { with Ndel and EcoR/ sites }\end{array}$ \\
\hline pGBKT7 GRASP55 & $\begin{array}{l}\text { GRASP55 was amplified by PCR using OSE196 } \\
\text { and oSE197 primers and cloned into pGBKT\& } \\
\text { vector using } \mathrm{Ncol} \text { and Notl sites }\end{array}$ \\
\hline pGST parallel I RAB-2 WT & $\begin{array}{l}\text { rab-2 was cloned into pGST parallel I vector } \\
\text { using Ncol and Xhol sites }\end{array}$ \\
\hline $\begin{array}{l}\text { pGST parallel I RAB-2 (Q65L) } \\
\text { DA }\end{array}$ & $\begin{array}{l}\text { rab-2 (Q65L) DA was cloned into pGST parallel } \\
\text { vector using } \mathrm{Ncol} \text { and } \mathrm{Xhol} \text { sites }\end{array}$ \\
\hline $\begin{array}{l}\text { pGST parallel I RAB-2 (S20N) } \\
\text { DN }\end{array}$ & $\begin{array}{l}\text { rab-2 (S20N) DN was cloned into pGST parallel } \\
\text { vector using } \mathrm{Ncol} \text { and } \mathrm{Xhol} \text { sites }\end{array}$ \\
\hline $\begin{array}{l}\text { pGST parallel I RAB-2 } \\
\text { (D122N) } n 501\end{array}$ & $\begin{array}{l}\text { rab-2 (D122N) } n 501 \text { was constructed using PCR } \\
\text { directed mutagenesis with oGQ165 and } \\
\text { oGQ166 primers and cloned into pGST parallel } \\
\text { vector using } \mathrm{Ncol} \text { and } \mathrm{Xhol} \text { sites }\end{array}$ \\
\hline $\begin{array}{l}\text { pGST parallel I RAB-2 (S149F) } \\
n 777\end{array}$ & $\begin{array}{l}\text { rab-2 (S149F) n777 was constructed using PCR } \\
\text { directed mutagenesis with oGQ167 and } \\
\text { oGQ168 primers and cloned into pGST parallel } \\
\text { vector using } \mathrm{Ncol} \text { and } \mathrm{Xhol} \text { sites }\end{array}$ \\
\hline $\begin{array}{l}\text { pGST parallel I RAB-2 (I11F) } \\
\text { ce365 }\end{array}$ & $\begin{array}{l}\text { rab-2 (I11F) ce } 365 \text { was constructed using PCR } \\
\text { directed mutagenesis with primers and cloned } \\
\text { into pGST parallel vector using Ncol and Xhol } \\
\text { sites }\end{array}$ \\
\hline prab-3::PH & $\begin{array}{l}\text { PH domain of PLCס was subcloned from pHD86 } \\
\text { using Kpnl site }\end{array}$ \\
\hline $\begin{array}{l}\text { prab-3::PH-PLCס-GFP (K30A, } \\
\text { K32A, W36N) }\end{array}$ & $\begin{array}{l}\text { K30A, K32A, W36N mutations were induced } \\
\text { using oGQ530 and oGQ531 primers and } \\
\text { fragment was exchanged in prab-3::PH- PLCס- } \\
\text { GFP construct }\end{array}$ \\
\hline prab-3::GFP-Cb5 & $\begin{array}{l}\text { rab-3 promoter was exchanged for myo-3 in } \\
\text { pmyo-3::GFP-Cb5 using Pstl and Kpnl sites }\end{array}$ \\
\hline prab-3::MANSII-GFP & $\begin{array}{l}\text { rab-3 promoter was exchanged for myo-3 in } \\
\text { pmyo -3::MANSII-GFP using Pstl and } K p n I \text { sites }\end{array}$ \\
\hline prab-3::RFP-عCOPI & $\begin{array}{l}\text { rab-3 promoter was exchanged for myo-3 in } \\
\text { pmyo -3::RFP-عCOPI using Pstl and Kpnl sites }\end{array}$ \\
\hline prab-3::2xFYVE domain-GFP & $\begin{array}{l}\text { Rab-3 promoter was exchanged for myo-3 in } \\
\text { pmyo -3::2xFYVE domain-GF using Pstl and } \\
\text { Kpnl sites }\end{array}$ \\
\hline prab-3::mCherry-rab-3 & $\begin{array}{l}\text { mCherry was amplified by PCR using oGQ137 } \\
\text { and oGQ139 and cut with Kpnl and Agel. rab-3 } \\
\text { Agel/Xhol fragment from L4440 RAB-3 vector } \\
\text { together with mCherry was cloned by three } \\
\text { fragment ligation into the pHU004 vector cut with } \\
\text { Kpnl and Xhol }\end{array}$ \\
\hline L4440 rab-3 & $\begin{array}{l}\text { rab-3 was amplified by PCR using oGQ230 and } \\
\text { oGQ231 primers and cloned into the vector } \\
\text { using Ncol and Xhol sites }\end{array}$ \\
\hline
\end{tabular}




\begin{tabular}{|l|l|}
\hline L4440 RAB-2 & rab-2 was cloned using Ncol and Xhol sites \\
\hline prab-3::mcherry-rab-2 & mCherry was amplified by PCR using oGQ137 \\
and oGQ139 and cut with Kpnl and Agel. rab-2 \\
Agel/Xhol fragment from L4440 RAB-2 vector \\
together with mCherry was cloned by three \\
fragment ligation into the pHU004 vector cut with \\
Kpnl and Xhol
\end{tabular}




\begin{tabular}{|l|l|}
\hline pIRES-eGFP RAB-2 (S20N) & S20N was subcloned using Ncol and Xhol sites \\
DN & into pIRES2-eGFP RAB-2 WT \\
\hline pIRES-eGFP RAB-2 (Q65L) & Q65L was subcloned using Ncol and Xhol sites \\
DA & into pIRES2-eGFP RAB-2 WT \\
\hline pcDNA4 RIC-19 & ric-19 was cloned into pcDNA4 vector using \\
& Kpnl and Xhol sites \\
\hline prab-3::mCherry-RAB-2 & S20N was subcloned into prab-3::mCherry-rab-2 \\
(S2ON) DN & using Agel and Xhol sites \\
\hline prab-3::mCherry-RAB-2 & Q78L was subcloned into prab-3::mCherry-rab-2 \\
(Q78L) DA & using Agel and Xhol sites \\
\hline prab-3::ric-19-yfp & ric-19 was subcloned into prab-3:::yfp vector \\
& using Kpnl and Notl sites \\
\hline
\end{tabular}

\subsection{Crosses and transgenic strains}

Crosses were performed using classical genetic approaches, and the progeny was genotyped by PCR and sequencing. Table 3 lists all of the strains used and generated in this study. Transgenic strains were generated by microinjection of DNA solutions into the distal gonad of young adult hermaphrodites as previously described (Mello et al., 1991). The injection mixes were adjusted to a total DNA concentration of $100 \mathrm{ng} / \mu \mathrm{l}$ by the addition of pBlueScript SKII (Agilent technologies). Table 4 lists all of the transgenic strains generated for this study, including the plasmid and coinjection marker concentration. As coinjection marker we used pRF4 (rol-6(su1006)), pttx3::gfp kindly provided by Craig C. Mello (Howard Hughes Medical Institute, Worcester, MA). and pttx-3::rfp a gift from Joshua Kaplan (Massachusetts General Hospital, Boston, MA). 
Table 3. List of strains used in this study

\begin{tabular}{|c|c|}
\hline Strain name & Genotype \\
\hline MT1093 & unc-108 (n501) \\
\hline MT1656 & unc-108 (n777) \\
\hline KG1279 & unc-108 (ce363) \\
\hline KG1281 & unc-108 (ce365) \\
\hline KG1900 & unc-108 (nu415) \\
\hline NM791 & $r a b-3(j s 49)$ \\
\hline VC461 & egl-3 (gk238) \\
\hline RB946 & ric-19 (ok833) \\
\hline \multirow[t]{2}{*}{ RB1131 } & lev-10 (ok1154) \\
\hline & nuls183 [punc-129::n/p-21-mvenus] \\
\hline GQ025 & $\begin{array}{l}\text { unc-108 (n777); } \\
\text { nuls183[punc-129::nlp-21-mvenus] }\end{array}$ \\
\hline GQ026 & $\begin{array}{l}\text { unc-108 (n501); } \\
\text { nuls183 [punc-129::n/p-21-mvenus] }\end{array}$ \\
\hline GQ142 & $\begin{array}{l}\text { unc-108 (nu415); } \\
\text { nuls183 [punc-129::nlp-21-mvenus] }\end{array}$ \\
\hline GQ143 & $\begin{array}{l}\text { egl-3 (gk238); } \\
\text { nuls183 [punc-129::nlp-21-mvenus] }\end{array}$ \\
\hline GQ144 & $\begin{array}{l}\text { egl-3 (gk238); unc-108 (nu415); } \\
\text { nuls183 [punc-129::nlp-21-mvenus] }\end{array}$ \\
\hline GQ145 & $\begin{array}{l}\text { egl-3 (gk238); unc-108 (n501); } \\
\text { nuls183 [punc-129::nlp-21-mvenus] }\end{array}$ \\
\hline NL4256 & $r r f-3(p k 1426)$ \\
\hline GQ146 & $\begin{array}{l}\text { unc-108 (n501); rrf-3(pk1426); } \\
\text { nuls183 [punc-129::nlp-21-mvenus] }\end{array}$ \\
\hline GQ147 & rrf-3(pk1426); nuls183 [punc-129::nlp-21-mvenus] \\
\hline GQ148 & $\begin{array}{l}\text { ric-19 (ok833); rrf-3(pk1426); } \\
\text { nuls183 [punc-129::nlp-21-mvenus] }\end{array}$ \\
\hline EG1285 & ox/s12[punc-47::gfp] \\
\hline GQ027 & unc-108 (n777); oxIs12[punc-47::gfp] \\
\hline \multirow[t]{2}{*}{ GQ028 } & unc-108 (n501); oxls12[punc-47::gfp] \\
\hline & nuls152[punc-129::gfp-snb-1] \\
\hline GQ029 & unc-108 (n777); nuls152[punc-129::gfp-snb-1] \\
\hline \multirow[t]{2}{*}{ GQ030 } & unc-108 (n501); nuls152[punc-129::gfp-snb-1] \\
\hline & arls37[pmyo-3::ssgfp] \\
\hline GQ112 & unc-108 (n501); arls37[pmyo-3::ssgfp] \\
\hline GQ113 & unc-108 (n777); arls37[pmyo-3::ssgfp] \\
\hline $\begin{array}{l}\text { GQ114 } \\
\text { TV199 }\end{array}$ & $\begin{array}{l}\text { unc-108 (ce365); arls37[pmyo-3::ssgfp] } \\
\text { wyls22/unc-86::afp-rab-3] }\end{array}$ \\
\hline GQ023 & $\begin{array}{l}\text { wyls22[unc-86::gfp-rab-3]; } \\
\text { gz100 [prab-3::mCherry-rab-2] }\end{array}$ \\
\hline DH1336 & b/s34 [prme-8::rme-8-gfp] \\
\hline GQ115 & unc-108 (n501); bls34 [prme-8::rme-8-gfp] \\
\hline GQ116 & unc-108 (n777); bls34 [prme-8::rme-8-gfp] \\
\hline GQ117 & unc-108 (ce365); b/s34 [prme-8::rme-8-gfp] \\
\hline NP745 & cdls40 [unc-122::gfp-cup-5] \\
\hline
\end{tabular}




\begin{tabular}{|c|c|}
\hline GQ118 & unc-108 (n501); cdls40 [unc-122::gfp-cup-5] \\
\hline GQ119 & unc-108 (n777); cdls40 [unc-122::gfp-cup-5] \\
\hline GQ120 & unc-108 (ce365); cdls40 [unc-122::gfp-cup-5] \\
\hline CB5600 & ccls4251; him-8(e1489) \\
\hline NL2003 & ric-19 (pk690) \\
\hline GQ121 & gz102[prab-3::gfp-cb-5; prab-3::mCherry-rab-2] \\
\hline GQ122 & $\begin{array}{l}\text { gz101[prab-3::mannosidasell-gfp; } \\
\text { prab-3::mCherry-rab-2] }\end{array}$ \\
\hline GQ123 & $\begin{array}{l}\text { gz103[prab-3::gfp-2xfyve domain; } \\
\text { prab-3::mCherry-rab-2] }\end{array}$ \\
\hline GQ124 & 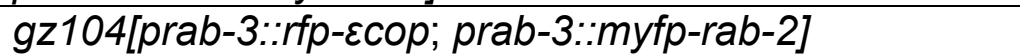 \\
\hline GQ125 & gz105[prab-3::mCherry-rab-3; prab-3::myfp-rab-2] \\
\hline GQ126 & $\begin{array}{l}\text { gz112[prab-3::mCherry-rab-2 (DN)S20N; } \\
\text { prab-3::ric-19-yfp] }\end{array}$ \\
\hline GQ127 & $\begin{array}{l}\text { gz113[prab-3::mCherry-rab-2 (WT); } \\
\text { prab-3::ric-19-yfp] }\end{array}$ \\
\hline GQ128 & $\begin{array}{l}\text { gz114[prab-3::mCherry-rab-2 (DA)Q65L; } \\
\text { prab-3::ric-19-yfp] }\end{array}$ \\
\hline GQ129 & $\begin{array}{l}\text { nuls183 [punc-129::nlp-21-mvenus]; } \\
\text { gz100[prab-3::mCherry-rab-2] }\end{array}$ \\
\hline GQ130 & $\begin{array}{l}\text { nuls183 [punc-129::nlp-21-mvenus]; } \\
\text { gz106[prab-3::mCherry-syn-6] }\end{array}$ \\
\hline GQ131 & $\begin{array}{l}\text { nuls183 [punc-129::nlp-21-mvenus]; } \\
\text { gz107[prab-3::mCherry-rab-5] }\end{array}$ \\
\hline GQ138 & $\begin{array}{l}\text { nuls183 [punc-129:::nlp-21-mvenus]; } \\
\text { gz108[prab-3::mCherry-rab-7] }\end{array}$ \\
\hline GQ139 & $\begin{array}{l}\text { nuls183 [punc-129::n/p-21-mvenus]; } \\
\text { gz109[prab-3::Imp-1-mCherry] }\end{array}$ \\
\hline GQ149 & $\begin{array}{l}\text { unc-108 (n501); nuls183 [punc-129::nlp-21-mvenus]; } \\
\text { gz106[prab-3::mCherry-syn-6] }\end{array}$ \\
\hline GQ150 & $\begin{array}{l}\text { unc-108 (n501); nuls183 [punc-129::n/p-21-mvenus]; } \\
\text { gz107[prab-3::mCherry-rab-5] }\end{array}$ \\
\hline GQ151 & $\begin{array}{l}\text { unc-108 (n501); nuls183 [punc-129::nlp-21-mvenus]; } \\
\text { gz108[prab-3::mCherry-rab-7] }\end{array}$ \\
\hline GQ152 & $\begin{array}{l}\text { unc-108 (n501); nuls183 [punc-129::nIp-21-mvenus]; } \\
\text { gz109[prab-3::Imp-1-mCherry] }\end{array}$ \\
\hline GQ153 & $\begin{array}{l}\text { nuls183 [punc-129:::n/p-21-mvenus]; } \\
\text { gz110[prab-3::mCherry-rab-5 (DA)Q78L] }\end{array}$ \\
\hline GQ154 & $\begin{array}{l}\text { unc-108 (n501); } \\
\text { nuls183 [punc-129::nlp-21-mvenus]; } \\
\text { gz110[prab-3::mCherry-rab-5 (DA)Q78L] }\end{array}$ \\
\hline GQ137 & $\begin{array}{l}\text { ric-19 (ok833); nuls183 [punc-129::nlp-21-mvenus]; } \\
\text { gz111[prab-3::ric-19] }\end{array}$ \\
\hline GQ136 & $\begin{array}{l}\text { nuls183 [punc-129::nlp-21-mvenus]; gz111[prab-3::ric- } \\
\text { 19] }\end{array}$ \\
\hline GQ135 & gz116[prab-3::ric-19-yfp] \\
\hline GQ133 & unc-108 (n501); gz116[prab-3::ric-19-yfp] \\
\hline GQ134 & unc-108 (n777); gz116[prab-3::ric-19-yfp] \\
\hline
\end{tabular}




\begin{tabular}{|l|l|}
\hline GQ132 & gz115[prab-2::gfp] \\
\hline DA509 & unc-31 (e928) \\
\hline
\end{tabular}

Table 4. Transgenic arrays used in this study

\begin{tabular}{|c|c|c|}
\hline Array name & Plasmid & Markers \\
\hline$g z 102$ & 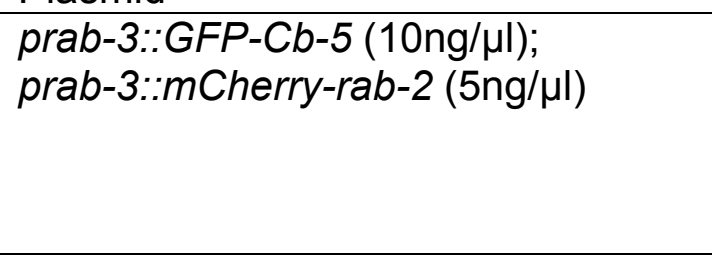 & $\begin{array}{l}\text { pRF4 } \\
(\text { rol-6(su1006)) } \\
(40 \mathrm{ng} / \mu \mathrm{l}) ; \\
\text { pttx-3::gfp } \\
(20 \mathrm{ng} / \mu \mathrm{l})\end{array}$ \\
\hline$g z 101$ & 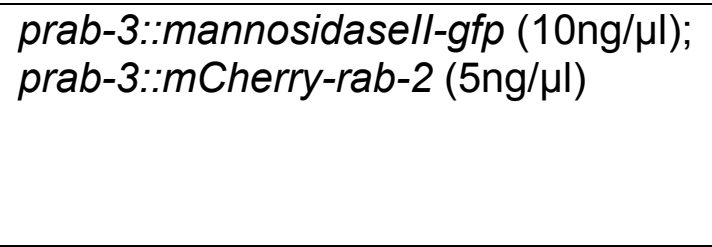 & 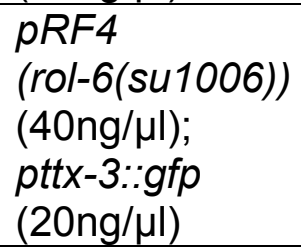 \\
\hline gz103 & $\begin{array}{l}\text { prab-3::gfp-2xfyve domain }(10 \mathrm{ng} / \mu \mathrm{l}) \\
\text { prab-3::mCherry-rab-2 }(5 \mathrm{ng} / \mu \mathrm{l})\end{array}$ & $\begin{array}{l}\text { pRF4 } \\
\text { (rol-6(su1006)) } \\
(40 \mathrm{ng} / \mu \mathrm{l}) ; \\
\text { pttx-3::gfp } \\
(20 \mathrm{ng} / \mu \mathrm{l})\end{array}$ \\
\hline gz104 & 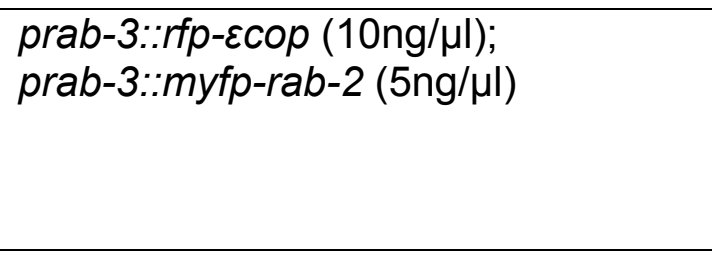 & $\begin{array}{l}\text { pRF4 } \\
(\text { (rol-6(su1006)) } \\
(40 \mathrm{ng} / \mu \mathrm{l}) ; \\
\text { pttx-3::gfp } \\
(20 \mathrm{ng} / \mu \mathrm{l})\end{array}$ \\
\hline$g z 105$ & 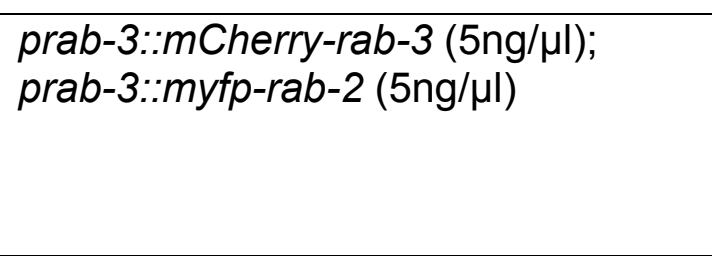 & $\begin{array}{l}\text { pRF4 } \\
(\text { rol-6(su1006)) } \\
(40 \mathrm{ng} / \mu \mathrm{ll}) ; \\
\text { pttx-3::gfp } \\
(20 \mathrm{ng} / \mu \mathrm{l})\end{array}$ \\
\hline$g z 100$ & 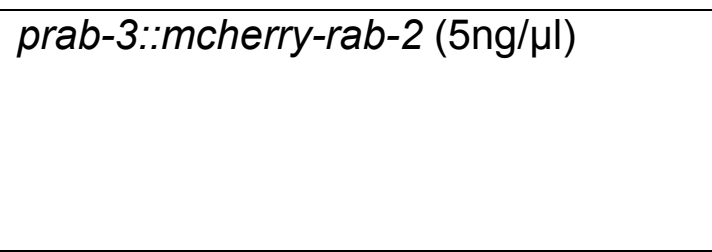 & $\begin{array}{l}\text { pRF4 } \\
(\text { rol-6(su1006)) } \\
(40 \mathrm{ng} / \mu \mathrm{ll}) ; \\
\text { pttx-3::gfp } \\
(20 \mathrm{ng} / \mu \mathrm{l})\end{array}$ \\
\hline$g z 106$ & prab-3::mCherry-syn-6 (10ng/Ml) & $\begin{array}{l}\text { pRF4 } \\
\text { (rol-6(su1006)) } \\
(40 \mathrm{ng} / \mu \mathrm{ll}) ; \\
\text { pttx-3::Ifp } \\
(20 \mathrm{ng} / \mu \mathrm{l})\end{array}$ \\
\hline gz107 & 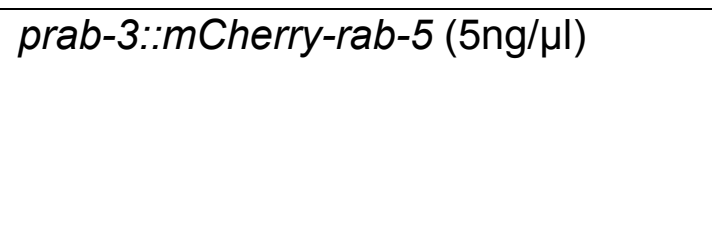 & 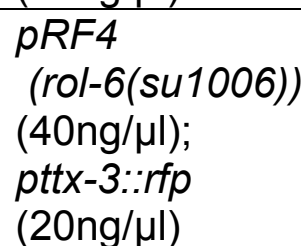 \\
\hline$g z 108$ & 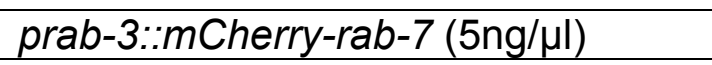 & pRF4 \\
\hline
\end{tabular}




\begin{tabular}{|c|c|c|}
\hline & & $\begin{array}{l}\text { (rol-6(su1006)) } \\
(40 \mathrm{ng} / \mu \mathrm{l}) ; \\
\text { pttx-3::rfp } \\
(20 \mathrm{ng} / \mu \mathrm{l})\end{array}$ \\
\hline$g z 109$ & prab-3::Imp-1-mCherry (20ng/ul) & $\begin{array}{l}\text { pRF4 } \\
\text { (rol-6(su1006)) } \\
(40 \mathrm{ng} / \mu \mathrm{l}) ; \\
\text { pttx-3::rfp } \\
(20 \mathrm{ng} / \mu \mathrm{l})\end{array}$ \\
\hline$g z 110$ & 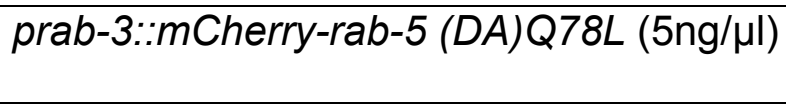 & $\begin{array}{l}\text { pttx-3::rfp } \\
(20 \mathrm{ng} / \mu \mathrm{l})\end{array}$ \\
\hline$g z 111$ & prab-3::ric-19 (20ng/ $\mu l)$ & $\begin{array}{l}\text { pttx-3::rfp } \\
(20 \mathrm{ng} / \mu \mathrm{l})\end{array}$ \\
\hline$g z 112$ & 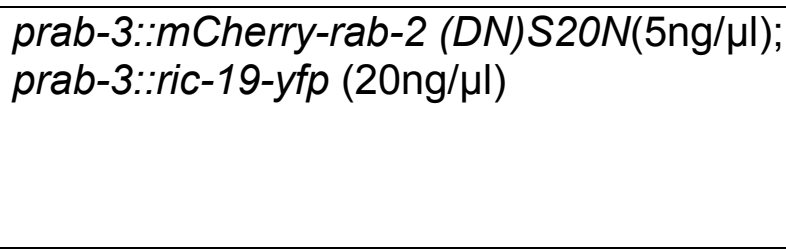 & $\begin{array}{l}\text { pRF4 } \\
\text { (rol-6(su1006)) } \\
(40 \mathrm{ng} / \mu \mathrm{l}) ; \\
\text { pttx-3::gfp } \\
(20 \mathrm{ng} / \mu \mathrm{l})\end{array}$ \\
\hline$g z 113$ & 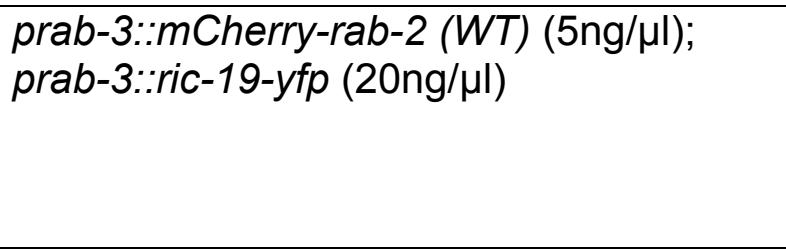 & $\begin{array}{l}\text { pRF4 } \\
\text { (rol-6(su1006)) } \\
(40 \mathrm{ng} / \mu \mathrm{l}) ; \\
\text { pttx-3::gfp } \\
(20 \mathrm{ng} / \mu \mathrm{l})\end{array}$ \\
\hline$g z 114$ & 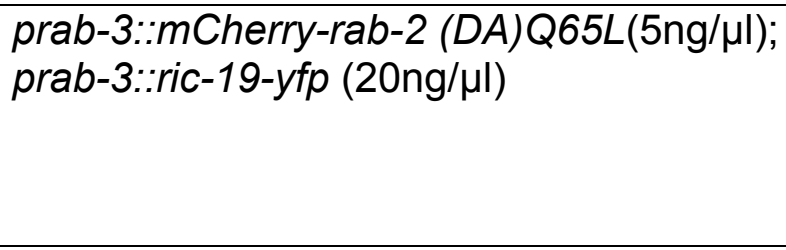 & $\begin{array}{l}\text { pRF4 } \\
\text { (rol-6(su1006)) } \\
(40 \mathrm{ng} / \mu \mathrm{l}) ; \\
\text { pttx-3::gfp } \\
(20 \mathrm{ng} / \mu \mathrm{l})\end{array}$ \\
\hline$g z 115$ & 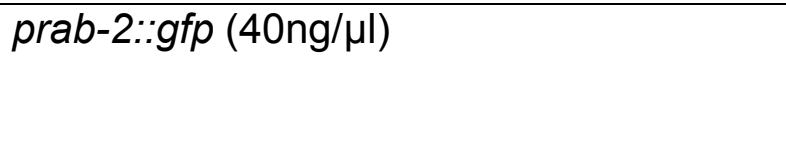 & $\begin{array}{l}\text { pRF4 } \\
(\text { rol-6(su1006)) } \\
(40 \mathrm{ng} / \mu \mathrm{l})\end{array}$ \\
\hline$g z 116$ & 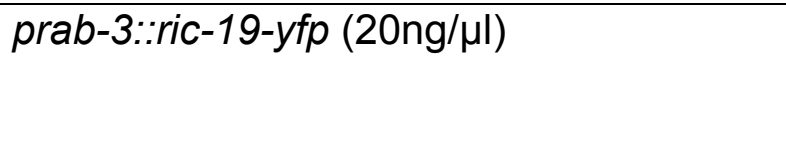 & $\begin{array}{l}\text { pRF4 } \\
(\text { rol-6(su1006)) } \\
(40 \mathrm{ng} / \mu \mathrm{l})\end{array}$ \\
\hline
\end{tabular}

\subsection{Fluorescence imaging and quantitative analysis}

Live animals were placed on $2 \%$ agarose pads and immobilized with $50 \mathrm{mM}$ sodium azide. All images were obtained on inverted microscope (SP2; Leica) using $63 \times N A=1.32$ oil immersion objective at $20^{\circ} \mathrm{C}$. Image stacks were captured and maximum intensity projections were obtained using Confocal software (Leica). 
For localization studies, images of neuronal cell bodies in ventral nerve cord were taken. For the dorsal nerve cord studies images were obtained as described (Sieburth et al., 2007). The posterior part of dorsal nerve cord was imaged in young adult animals when oriented towards the objective, using the same settings for all the images obtained. For the coelomocyte imaging, posterior coelomocyte was imaged in young adults when oriented laterally. Maximum stack projections were tresholded and analyzed in ImageJ software (http:IIrsb.info.nih.gov/ij/; National Institute for Health, Bethesda, MD) as described previously (Sieburth et al., 2007). For the vesicular structure diameter quantification, stack through entire cells were obtained using the same magnification for all cells and the diameter was determined for each vesicle observed using ImageJ software. The data were analyzed in ImageJ software and normalized to wild type.

\subsection{Protein expression and purification}

rab-2 full sequence was cloned into Ncol and Xhol sites of pGST parallel I vector (Sheffield et al., 1999). The protein was produced in E. coli BL21 star (DE3) strain (Invitrogen) by inducing expression with $1 \mathrm{mM}$ isopropyl $\beta-D-$ thiogalactopyranoside (IPTG). The protein was purified by selective binding to

Glutathione Sepharose column (GE Healthcare). The IPTG induced cells were resuspended in buffer containing $50 \mathrm{mM}$ Tris- $\mathrm{HCl}$ pH7, 150mM NaCl, $1 \mathrm{mM}$ EDTA, 2mM DTT. The solution was sonicated on ice and centrifuged to remove the debris at $10000 \mathrm{~g}$ for 30 minutes. The supernatant was incubated with GST-Sepharose for one hour on room temperature, subjected to 3-4 
rounds of washes with PBS and eluted with $50 \mathrm{mM}$ Tris- $\mathrm{HCl} \mathrm{pH} 8,150 \mathrm{mMNaCl}$ 20mM reduced glutathione and Tween 20.

\subsection{Antibody production and Western blotting}

Ten mice were immunized with $50 \mu \mathrm{g}$ of purified fusion protein in PBS using complete Freund's adjuvant (Sigma). Booster injections were given thereafter every forth week with the same amount of antigen in incomplete Freund's adjuvant (Sigma). 30 days after the last injection the whole blood was collected. To obtain polyclonal mouse antibodies against RAB-2, the sera were combined and affinity purified against RAB-2 fusion protein as previously described (Eimer et al., 2007). Monoclonal antibodies against RAB-2 were generated subsequently according to standard protocols.

For RAB-2 and RIC-19 protein quantification, worms of different unc-108 strains were grown to near starvation and harvested with M9 buffer. Worms were then pelleted and lysed in homogenization buffer (20mM HEPES pH 7.4;

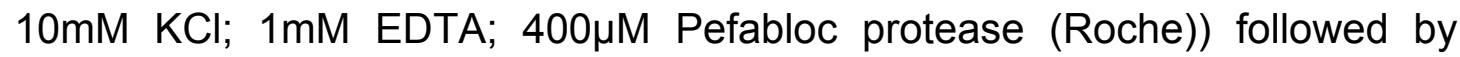
several rounds of sonication on ice. Nuclei and debris were removed by centrifuging the lysate at $500 \mathrm{~g}$ for $10 \mathrm{~min} .15 \mu \mathrm{g}$ of total protein was loaded and separated on $15 \%$ SDS polyacrylamide gel, blotted onto nitrocellulose membrane and probed against RAB-2 and RIC-19 (a gift from Marc Pilon) primary antibody using 1:2000 and 1:5000 dilutions respectively and a horse raddish coupled secondary antibody (Jacksons Laboratory, Bar Harbor, ME) The signal was revealed with Super Signal West Dura substrate (Pierce, 
Rockford, II). The images were quantified using ImageJ software and the data was normalized to tubulin.

\subsection{Cell culture}

HeLa cells were grown in DMEM medium supplemented with $10 \%$ FBS, 2 mM glutamine, $100 \mathrm{U} / \mathrm{ml}$ penicillin and $10 \mu \mathrm{g} / \mathrm{ml}$ streptomycin, in a $5 \% \quad \mathrm{CO}_{2}$ incubator at $37^{\circ} \mathrm{C}$. Cells were transfected in $35 \mathrm{~mm}$ tissue culture dishes as described (Sambrook et al., 1989). After 16 hours cells were washed in PBS, collected in PBS buffer containing 10mM EDTA and extracted in lysis buffer (50mM HEPES, pH7.4, 1\% Triton X-100, 100mM NaCl, $5 \mathrm{mM} \mathrm{MgCl}, 1 \mathrm{mM}$ PMSF and $10 \mathrm{mM} \mathrm{Na-phosphate)} \mathrm{for} 20 \mathrm{~min}$. at $4^{\circ} \mathrm{C}$. The protein extracts were loaded and analyzed on $15 \%$ SDS polyacrylamide gel. Immunoprecipitation was performed as described (Buffa et al., 2008)

\subsection{GTPase and GTP affinity assays}

The GTPase assay was performed as previously described by Stenmark et al. with modifications (Stenmark et al., 1994a). Purified proteins were dialyzed in buffer A (20mM Tris- $\mathrm{HCl}, \mathrm{pH} 7.8,100 \mathrm{mM} \mathrm{NaCl}, 5 \mathrm{mM} \mathrm{MgCl}_{2}, 1 \mathrm{mM} \mathrm{Na}-$ phosphate and 10mM 2-mercaptoethanol) and incubated with immobilized Glutathione agarose (Pierce) for $2 \mathrm{~h}$ at $4^{\circ} \mathrm{C}$. Bound nucleotide was eluted washing once with buffer $\mathrm{C}(20 \mathrm{mM}$ Tris- $\mathrm{HCl}, \mathrm{pH} 7.8,100 \mathrm{mM} \mathrm{NaCl}, 5 \mathrm{mM}$ $\mathrm{MgCl}_{2}, 1 \mathrm{mM}$ Na-phosphate, 2mM EDTA and 10mM 2-mercaptoethanol) and then twice in ice cold buffer A. 1pmol of immobilized GST tagged proteins was then mixed with $1 \mathrm{pmol}\left[\alpha^{-32} \mathrm{P}\right] \mathrm{GTP}$ in $20 \mu \mathrm{l}$ of buffer $\mathrm{A}$ and incubated at $4{ }^{\circ} \mathrm{C}$ for 10 minutes. $50 \mu$ l of buffer $A$ was then added to each sample and aliquots 
were taken at various time points. After incubation, proteins were washed three times in ice cold buffer A and $8 \mu$ l of buffer B was added $(0.2 \%$ SDS, 2mM EDTA, 10mM GDP, 10mM GTP, pH7.5). The samples were heated on $70^{\circ} \mathrm{C}$ for 2 minutes and $2 \mu \mathrm{l}$ aliquots were spotted on PEI cellulose membrane for thin layer chromatography in $0.6 \mathrm{Na}$-phosphate buffer, $\mathrm{pH} 3.5$ and the radioactivity spots were visualized and analysed in using ImageJ software. GTP affinity assay was performed as described (Shapiro et al., 1993). The bound nucleotide was eluted as before. Different alleles of RAB-2 (100 nM)

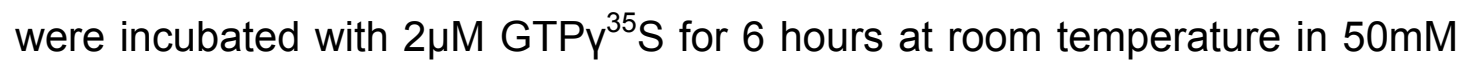
Hepes-KOH (pH7.4), $150 \mathrm{KCl}, 1 \mathrm{mM}$ EDTA, 1mM DTT). After binding, 100 fold excess of unlabelled GTP was added, and $50 \mu \mathrm{l}$ aliquots were collected at various time points, extensively washed in 20mM Tris- $\mathrm{HCl}(\mathrm{pH} 8.0), 100 \mathrm{mM}$ $\mathrm{NaCl}, 25 \mathrm{mM} \mathrm{MgCl}_{2}$ buffer. The radioactivity associated with the proteins was determined by scintillation counting.

\subsection{RNAi}

RNAi by feeding was performed as previously described (Kamath et al., 2001). L4440 vector caring the ric-19 gene or the empty L4440 vector was transformed into E.coli HT115 (DE3) strain. The overnight culture, grown in Luria broth media with $100 \mu \mathrm{g} / \mathrm{ml}$ ampicillin was used for seeding NGM plates containing $50 \mu \mathrm{g} / \mathrm{ml}$ ampicillin and 1mM IPTG. Ten L4 larvae of strains with rrf-3 mutant background were transferred onto the plates, left to lay eggs for $12 \mathrm{~h}$ and then transferred to a new plate. The progeny was analyzed. 


\subsection{Electron microscopy}

High pressure freezing / Freeze substitution. A $100 \mu \mathrm{m}$ deep aluminium platelet (Microscopy Services, Flintbek) was filled with E.coli OP50 suspension. About 20 young adult worms were transferred into the chamber and immediately frozen using a BalTec HPM 10. Freeze substitution was carried out in a Leica EM AFS or AFS2. For morphological investigations incubations were at $-90{ }^{\circ} \mathrm{C}$ for $100 \mathrm{~h}$ in $0,1 \%$ tannic acid, $7 \mathrm{~h}$ in $2 \% \mathrm{OsO}_{4}$, and at $-20{ }^{\circ} \mathrm{C}$ for $16 \mathrm{~h}$ in $2 \% \mathrm{OsO}_{4}$, followed by embedding in EPON at RT. For immunostaining incubations were at $-90^{\circ} \mathrm{C}$ for $100 \mathrm{~h}$ in $1,5 \%$ uranyl acetate, followed by embedding in Lowicryl HM20 at $-45^{\circ} \mathrm{C}$ (Rostaing et al., 2006) (all solutions $\mathrm{w} / \mathrm{v}$ in dry acetone).

Microscopy. For morphological investigations $50 \mathrm{~nm}$ sections were cut using a Leica UC6 ultramicrotome and transferred on Formvar-coated copper slotgrids. The grids were placed for $10 \mathrm{~min}$ on drops of $4 \%(\mathrm{w} / \mathrm{v})$ uranyl acetate in $75 \%$ methanol and then washed in destilled water. After air drying the grids were placed on lead citrate (Reynolds, 1963) for $2 \mathrm{~min}$ in a $\mathrm{CO}_{2}$-free chamber, and rinsed three times in destilled water. For immunostaining $85 \mathrm{~nm}$ sections were transferred on Formvar-coated copper slot-grids. The grids were placed on blocking buffer $(5 \%(\mathrm{w} / \mathrm{v})$ skim milk in BPST (PBS containing 0,025 \% Tween-20)) for $1 \mathrm{~h}$ and then incubated on the first antibody (anti-rab-2 mouseserum 1:50, or anti-GFP-antibody 1:100 in blocking buffer). The grids were washed three times on PBST and incubated on the secondary antibody for 45 $\min$ (10 nm goat-anti-mouse-gold, diluted 1:50 in blocking buffer). Final washing was two times on PBST and two times on destilled water. The grids were stained on $6 \%$ phosphotungstic acid $(\mathrm{pH} 7)$ for 20 min, followed by $4 \%$ 
(w/v) uranyl acetate in $75 \%$ methanol for $10 \mathrm{~min}$, and washed in destilled water. Micrographs were taken with a $1024 \times 1024$ CCD detector (Proscan CCD HSS 512/1024; Proscan Electronic Systems, Scheuring, Germany) in a Zeiss EM 902A, operated in the bright field mode.

Estimation of vesicle distribution at the presynaptic density. Osmium stained C. elegans worms were cut across near behind the pharynx and micrographs of synapses were taken at a magnification of 50000x. By a semiautomated process (manuscript in preparation) a rectangular grid containing 10 bins of each $50 \mathrm{~nm}$ was placed at the PD. Synaptic vesicles included in the grid were counted and the percentage of vesicles occurring in each bin was calculated. Of each individual dense core vesicle the distance to the membrane and to the PD was measured and displayed as cloud diagram.

Distinction of gold labeling at the Golgi. Electron micrographs of either intestinal or neuronal Golgi stacks were analysed by placing a rectangle, subdivided three equal parallel areas, accurately around the Golgi stack, ignoring any separated vesicular structures. Cis and trans side were defined either by the bending direction of the cisternae (for intestinal Golgi's, convex site as cis) or by the orientation of the Golgi stack relative to the nucleus (neurons, cis facing to nucleus). All gold particles were counted in each third of the rectangle, assigning the label to cis-, median or trans-Golgi.

\subsection{Peptidomics}

Endogenous peptides were extracted using a slightly modified protocol as previously described (Husson et al., 2005). Briefly, a mixed culture of nematodes was placed in $5 \mathrm{~mL}$ of an ice cold extraction solvent 
(methanol/water/acetic acid, 90/9/1). After homogenization, sonication and filtration, the pellet was discarded. The methanol was evaporated in order to re-extract the resulting aqueous solution with ethyl acetate and n-hexane to remove the lipids. Finally, the peptide extract was desalted by solid phase extraction using an Oasis HLB cartridge (10 mg, Waters, Milford, MA). Just prior to HPLC analysis, peptide sample was lyophilized and reconstituted in $500 \mu \mathrm{L}$ of water containing $4 \%$ of $\mathrm{CH}_{3} \mathrm{CN}$ and $0.1 \%$ trifluoroacetic acid (TFA) and filtered (22 $\mu \mathrm{m}$, Ultrafree-MC, Millipore Corporation, Bedford, MA). Peptides were separated on a Symmetry C18 column $(2.1$ x 150 mm, $3.5 \mu \mathrm{m}$, Waters) using a linear gradient from $4 \% \mathrm{CH}_{3} \mathrm{CN}$ in $0.1 \%$ aqueous TFA to $52 \%$ $\mathrm{CH}_{3} \mathrm{CN}$ in $0.1 \%$ aqueous TFA in 60 minutes at a flow-rate of $300 \mu \mathrm{L}$ per minute to generate 60 fractions. The peptide content of each fraction was monitored by matrix-assisted laser desorption ionization time-of-flight mass spectrometry (MALDI-TOF MS) on an Ultraflex II instrument (Bruker Daltonics, $\mathrm{GmbH}$, Bremen, Germany) in positive ion, reflectron mode using a-cyano-4hydroxy cinnamic acid as matrix. Spectra were recorded in MS mode within a mass range from m/z 500 to $\mathrm{m} / \mathrm{z} 4000$ and were automatically processed (background subtraction, smoothing and peak picking) using FlexAnalysis software (Bruker Daltonics) to generate peak list files. The obtained peak lists from all fractions were compared with the theoretical masses of previously identified and predicted NLP and FLP neuropeptides to construct a peptide profile. 


\section{Results}

\subsection{C. elegans UNC-108 is a homologue of human Rab2 and is predominantly expressed in the nervous system}

In order to find new Rab members involved in the unique membrane trafficking functions of neurons we analyzed the expression pattern of all Rab GTPases in C. elegans. For this purpose, transgenes expressing GFP under promoters of genes for different Rab GTPases were generated. The tissue specific activation of the promoter as well as the promoter strength, regulate the GFP expression levels. We found that UNC-108, which shows $92 \%$ identity to human Rab2 (Fig. 12) is ubiquitously expressed, predominantly in the nervous system (Fig. 10). This suggests an important role of UNC108/RAB-2 in neuronal trafficking. In agreement with this, unc-108/rab-2 mutants have been shown to exhibit a slow locomotion phenotype, indicative of defects in neurotransmission at the neuromuscular junction (Simmer et al., 2003).

To asses whether $C$. elegans RAB-2 is a true homolog of human Rab2, we analyzed the interaction of $C$. elegans RAB-2 with known interactors of mammalian Rab2. 

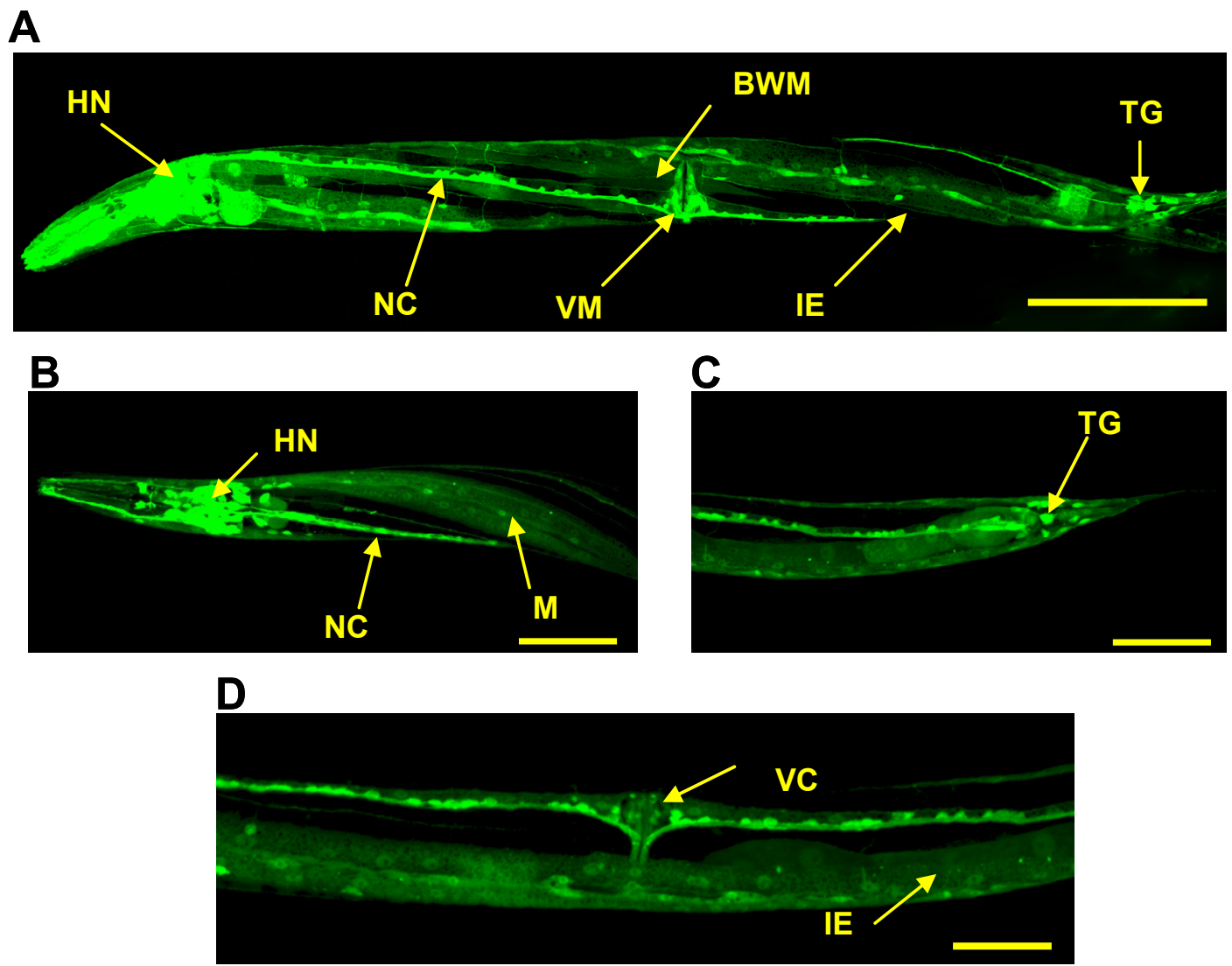

Figure 10. unc-108 shows ubiquitous expression with high expression levels in the nervous system.

(A) and (B) GFP was expressed under unc-108/rab-2 promoter. rab-2 is expressed in the body wall muscle (BWM), but shows strong expression levels in the nervous system (HN head neurons, NC - nerve cord, TG - tail ganglia) and vulva muscle (VM). Scale bar 20 $\mu \mathrm{m}$.

(C) Expression of rab-2 in vulval cells (VC) and intestinal epithelium (IE). Scale bar 30 $\mu$ m.

In mammalian system, Rab2 interacts with Golgi matrix proteins, proteins that are responsible for preserving the Golgi morphology and facilitating the transport of carrier vesicles by keeping them closer to the target membrane. Specifically, activated Rab2 was shown to interact with the Golgi matrix protein Golgin45. Rab2 and Golgin45 then form complex with GRASP55 (Short et al., 2001). We show here that only the activated, GTP bound form, of 
RAB-2 interacts with GOLGIN-45 in the yeast two hybrid system in C. elegans (Fig. 11). This suggests that not only the sequence of RAB-2 is conserved, but also the known interaction partner. Additionally, this also makes it likely that Rab2 function is conserved between $C$. elegans and the mammalian system.

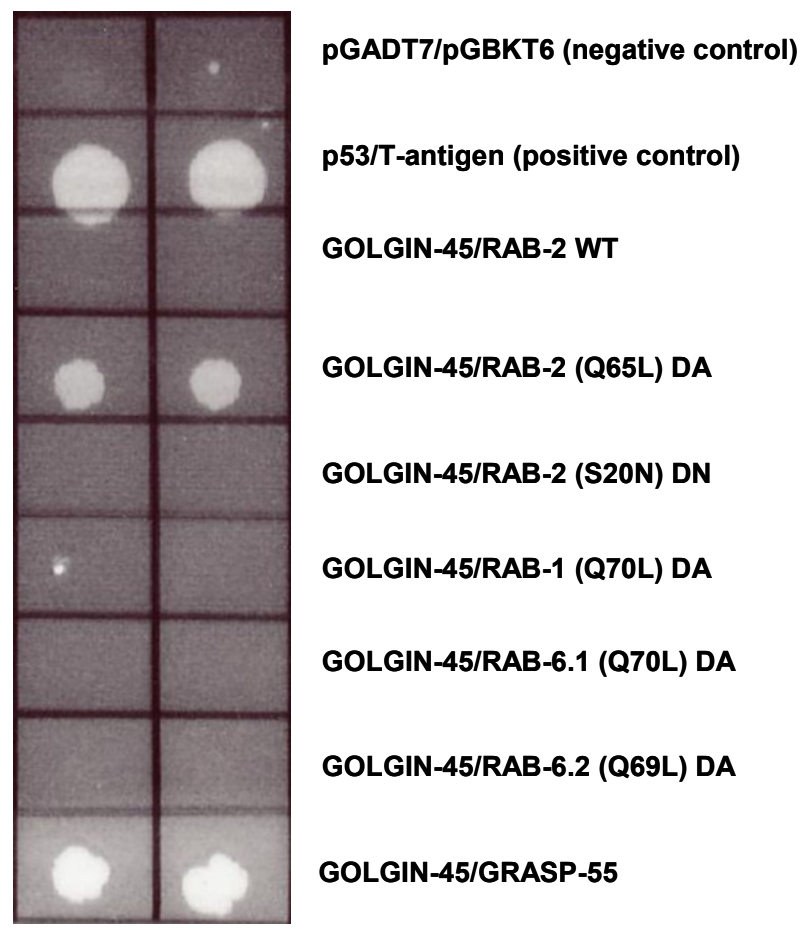

Figure 11. Golgi matrix protein GOLGIN-45 is an effector of RAB-2 in C. elegans.

Yeast two hybrid shows that GOLGIN-45 interacts specifically with activated GTP bound form of RAB-2. Furthermore, GOLGIN-45 interacts with GRASP-55. This interaction leads to the same complex formation as in mammalian system (Short et la., 2001). As a positive control interaction of p53 with $\mathrm{T}$ antigen was used.

In $C$. elegans, both dominant and recessive mutations in rab-2 gene have been isolated. In some cases they express the same and in some the unique phenotypes, suggesting that the different mutations in RAB-2 affect protein properties differently. Two alleles, unc-108 (n501) and (n777) are dominant and thus may resemble gain of function alleles. D122N (n501) and S149F 
(n777) are missense mutations that reside within the conserved domains G2 and G3, respectively, that are required for binding of the guanine moiety of the guanine nucleotide cofactors (GTP or GDP) (Fig. 12). Recessive loss-offunction alleles of unc-108/rab-2 have also been identified containing the missense mutations C213S (ce363) and I11F (ce365) (Chun et al., 2008; Lu et al., 2008; Mangahas et al., 2008) or a deletion nu415 that removes the Cterminal 66 amino acids of the protein and is not detectable on Western bots (Chun et al., 2008).

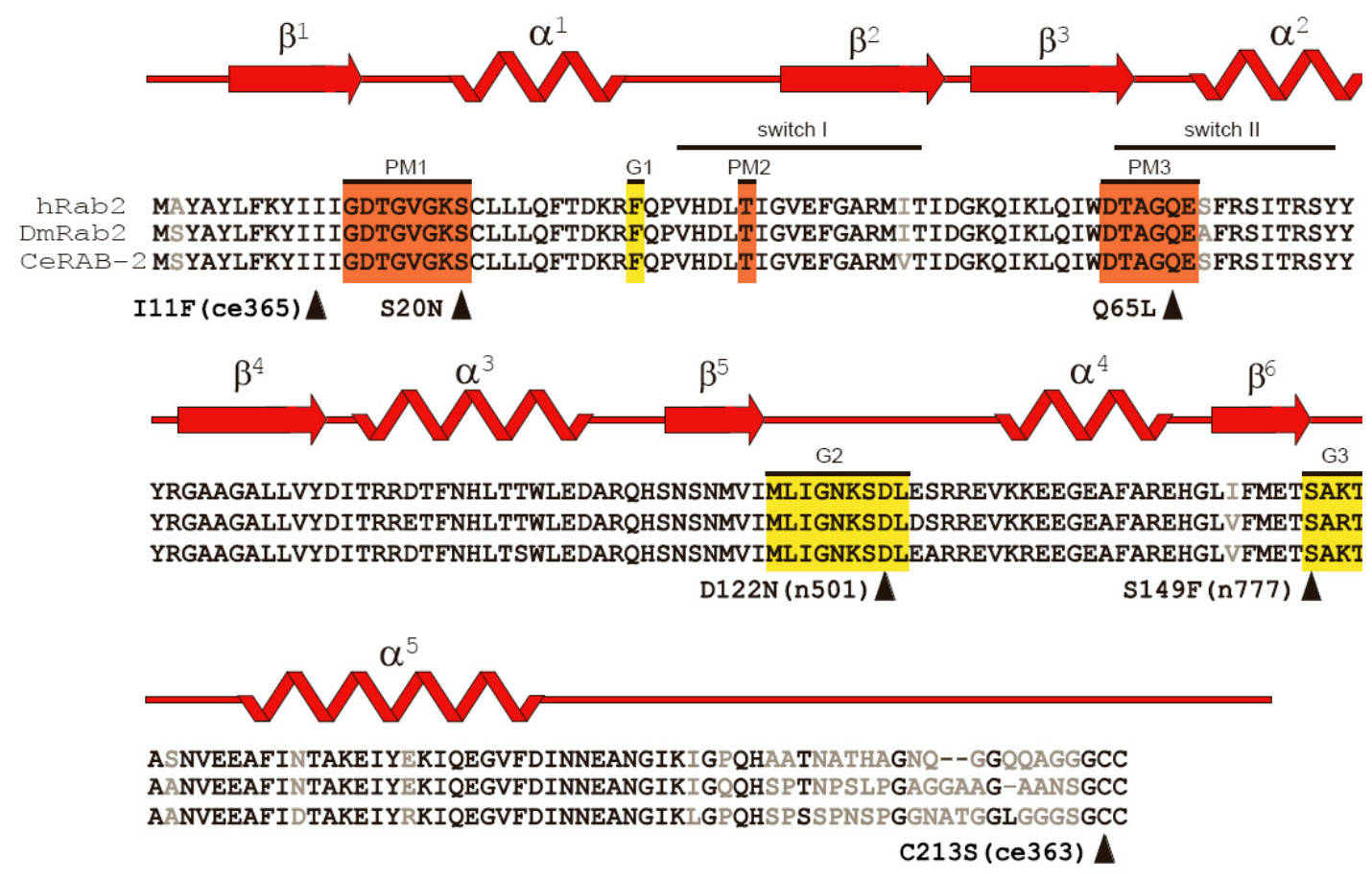

Figure 12. UNC-108 is a homologue of human Rab2.

Sequence alignment of $C$. elegans UNC-108/RAB-2 with $H$. sapiens (hRab2A, NP_002856) and D. melanogaster RAB-2 (DmRab2, NP_477090). Red boxes label domains responsible for phosphate and $\mathrm{Mg}^{2+}$ binding. Yellow boxes represent domains that bind guanine moiety. The locations of the unc-108/rab-2 point mutations within the protein are indicated by arrowheads. The conserved mutations rendering RAB-2 constitutively inactive GDP bound (S20N) and constitutively active GTP bound form (Q65L) are shown (Tisdale, 1999). 
We first wanted to see how the mutations affect the protein properties causing the phenotypes observed in unc-108 mutants. To test whether the mutations affect the protein stability, we probed the mixed staged protein extracts of different unc-108 strains against the monoclonal RAB-2 antibody. RAB-2 could be detected in all strains. However, for the $n 777$ allele, the RAB-2 protein levels were reduced to $20 \%$ of wild type (Fig. 13). This suggests that exchange of serine for phenylalanine at the position 149 may affect not only the biochemical properties of the protein, but also its stability.
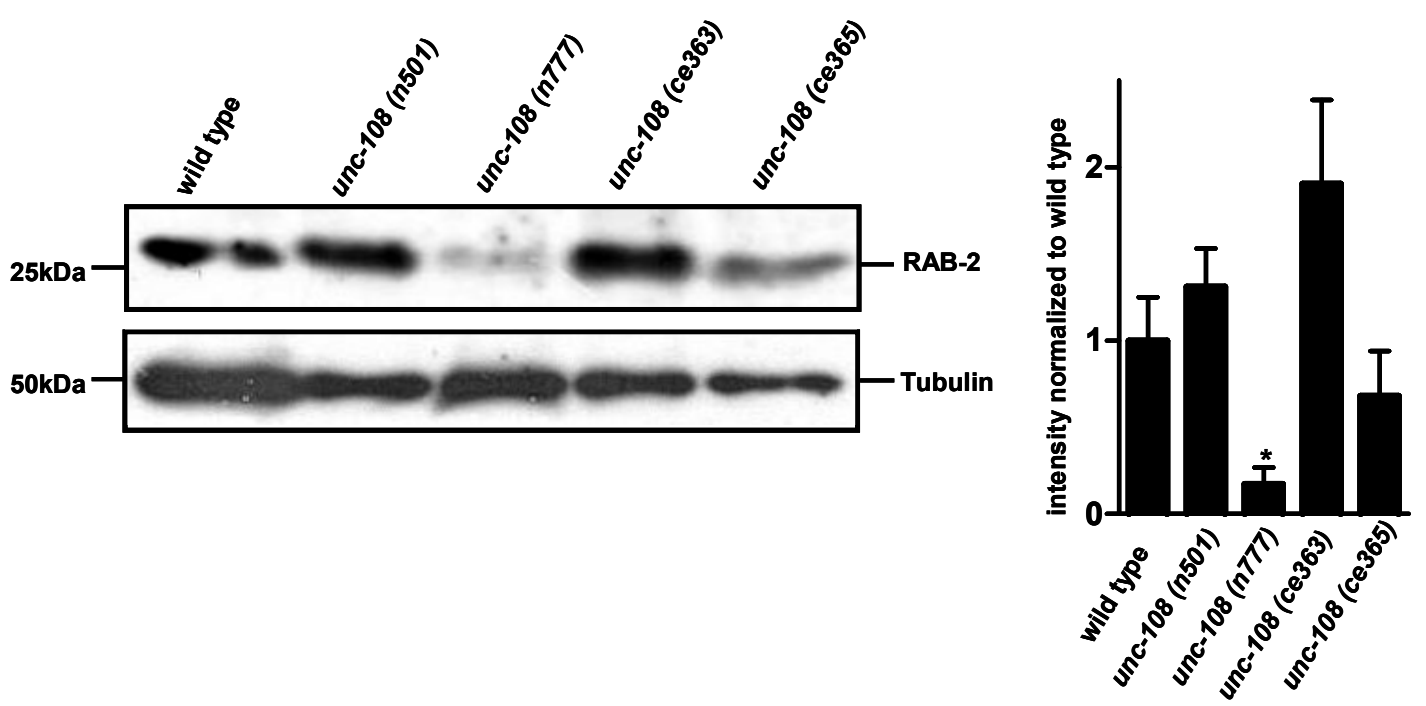

Figure 13. Exchange of Serine at the position 149 for Phenylalanine changes the stability of the protein.

Left - Western blot of protein extracts from dominant, $n 501$ and $n 777$, and recessive, ce363 and ce365 unc-108 alleles probed with polyclonal mouse anti-RAB-2 antibodies. Tubulin loading control is also shown. Right - Quantification of RAB-2 protein levels on Western blots of unc-108 mutants normalized against Tubulin. $\quad\left({ }^{*} p<0.05\right.$, Student's t-test, error bar=SEM; $\mathrm{N}=4)$.

Our protein stability analysis suggests it is unlikely that differences in stability could alone account for the functional defects common to all of the unc- 
108/rab-2 mutants. To analyze how the dominant active mutations $n 777$ and n501 affect RAB-2 protein function, we expressed wild type RAB-2 and its various mutant forms (see figure 12) in E. coli as Glutathion-S-transferase (GST) fusion proteins. After affinity purification, we determined the biochemical properties of each GST-RAB-2 fusion protein including efficiency of GTP hydrolysis and affinity for the cofactor GTP of each GST-RAB-2 fusion protein. As a control we used the RAB-2 mutants S20N (impaired in nucleotide exchange and reduced affinity for GTP) and Q65L (dominantly active due to a lack of GTPase activity but not GTP binding) (Spinosa et al., 2008; Tisdale, 1999).

Both dominant RAB-2 mutations (S149F) $n 777$ and (D122N) $n 501$ affect two loops that coordinate the guanine nucleotide, therefore, it is likely that GTP binding is affected (Fig. 14).

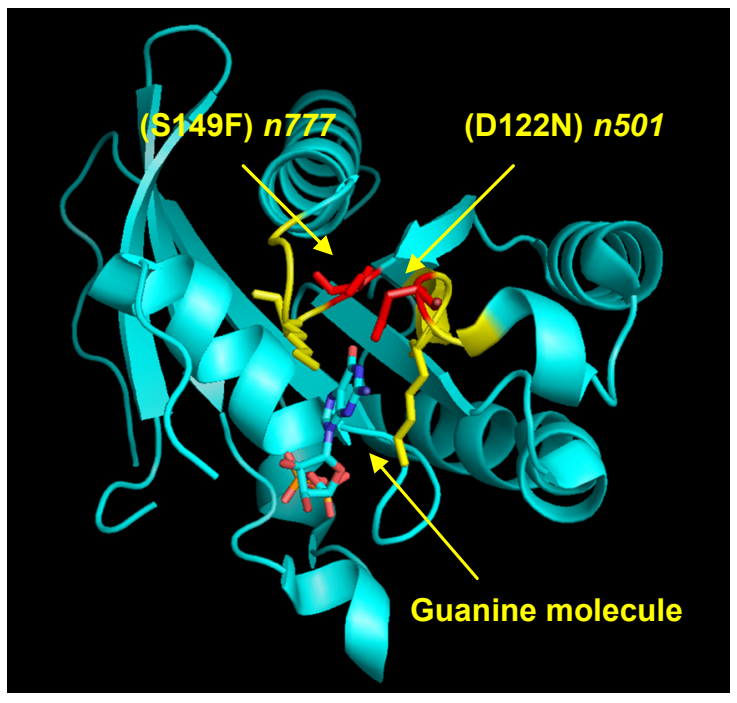

Figure 14. (S149F) $n 777$ and (D122N) n501 mutations affect the loop responsible for binding of guanine molecule

Structure of RAB-2 protein with guanine binding domain labeled in yellow. Red are the altered amino acids in dominant unc-108 mutants, which lay in the region responsible for binding of guanine molecule (arrow head). 
To test the GTPase activity of different rab-2 alleles, we preincubated the purified proteins with radioactively labeled GTP and measured the ratio of GTP versus the entire amount of guanine nucleotides at various time points.

A
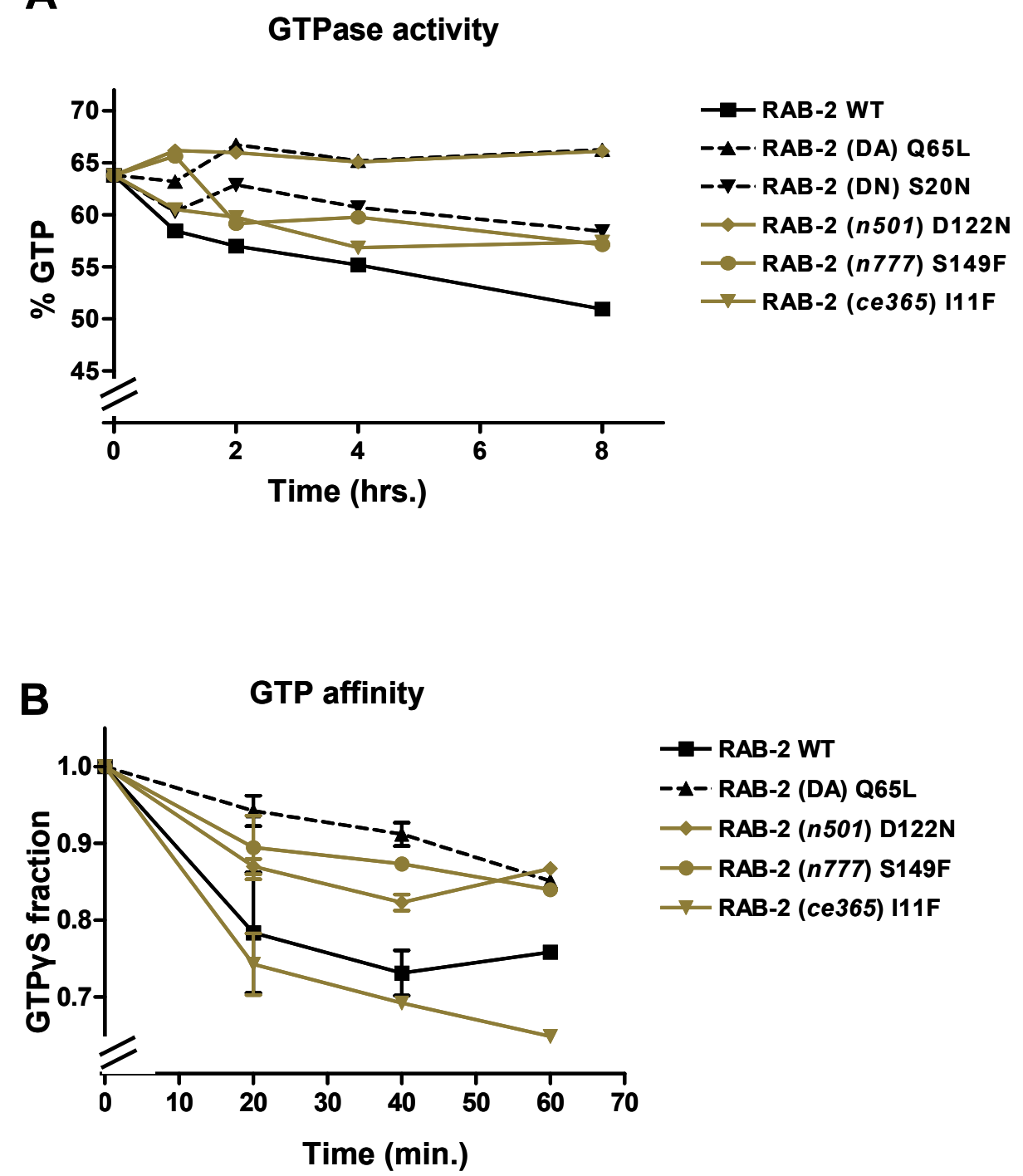

Figure 15. (S149F) $n 777$ and (D122N) $n 501$ mutations lead to increased affinity for GTP, but only (D122N) $n 501$ leads to reduced GTPase activity

(A) GTPase assay using recombinant RAB-2 proteins to determine the GTP hydrolysis rates of mutant RAB-2 ( $\mathrm{N}=3$ per time point).

(B) GTP affinity measurement of RAB-2 recombinant protein using nonhydrolyzable GTPYS (error bar=SEM, $\mathrm{N}=9$ per time point). 
GTP affinity was quantified by incubating the proteins with nonhydrolizable GTP form, GTPYS and measuring the amount of GTPYS still bound to the protein in different time points. While both mutations lead to an increase in the affinity for GTP (Fig. 15A) only the (D122N) n501 mutation prevents the hydrolysis of GTP (Fig. 15B). Thus, the (D122N) n501 mutant RAB-2 behaves similar to the constitutively active Q65L mutant. Therefore, RAB-2 $n 777$ and $n 501$ alleles can be considered constitutively active. Thus, biochemically $n 777$ and $n 501$ can be clearly distinguished from the recessive loss-of-function mutation (I11F) ce365, which displays wild type like GTP affinity and hydrolysis rates (Fig. 15A and B).

\section{2 unc-108/rab-2 mutants are aldicarb resistant}

unc-108 (n501) and (n777) mutations have been shown to lead to dominant movement defects (Chun et al., 2008; Park and Horvitz, 1986; Simmer et al., 2003) (Fig. 16). Both loss and gain of function mutations cause similar

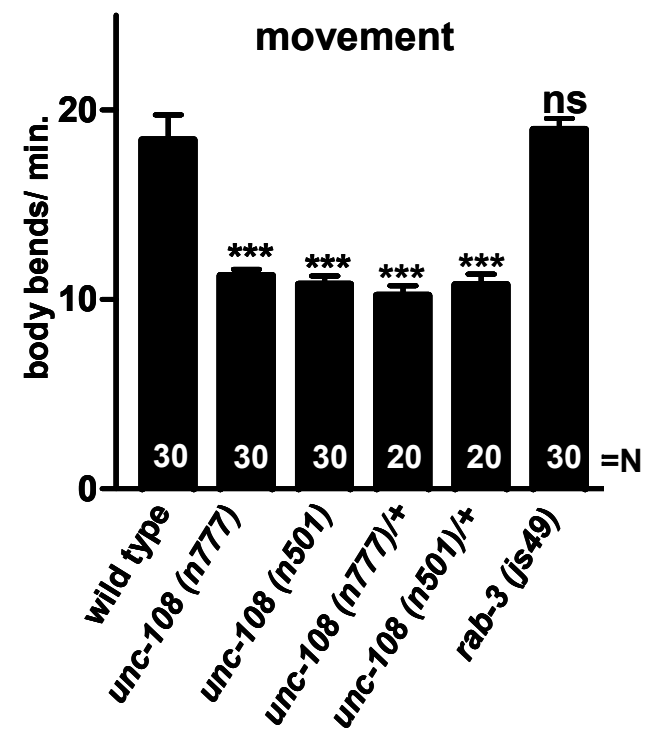

Figure 16. Gain-of-function unc-108 mutants show a dominant uncoordinated phenotype unc-108 mutants have a movement defect, and $n 501$ and $n 777$ mutations are dominant for the movement phenotype. (Number of animals analyzed is indicated in the graph bars, error bar is SEM, ${ }^{* * *} p<0.005$, Student's t-test). 
movement defects suggesting impairment in neurotransmission at the neuromuscular junction, but only gain-of-function mutants have a dominant phenotype.

Fast neurotransmission at the neuromuscular junction in $C$. elegans is mediated via acetylcholine. Acetylcholine signaling can be studied in $C$. elegans using an acetylcholine esterase inhibitor aldicarb (Mahoney et al., 2006). By inhibiting the esterase, aldicarb leads to accumulation of acetylcholine at the neuromuscular junction, hyperstimulation of the body wall muscle and paralysis of worms. Therefore, sensitivity to aldicarb is dependent on the amount of acetylcholine secreted and/or responsiveness of the postsynaptic membrane. unc-108 n777 and n501 mutants show delayed response to aldicarb when compared to wild type (Fig. 17A). Resistance of $n 501$ and $n 777$ alleles to aldicarb is comparable to resistance of rab-3 mutants that have been shown to have defects in SV tethering (Nonet et al., 1997, Gracheva et al., 2008). To exclude that aldicarb resistance comes from impairment in the postsynaptic side, we looked at the responsiveness of the mutants to an antihelminthic drug levamisole. Levamisole is an acetylcholine receptor agonist and at high concentration leads to paralysis of worms. The responsiveness of worms therefore depends on the number and clustering of the acetylcholine receptors of the muscles. unc-108 n501 and $n 777$ mutants displayed wild type response to increasing concentrations of levamisole (Fig. 17B) The sensitivity is clearly distinguishable from levamisole resistance of lev-10 mutants, lacking a protein needed for acetylcholine receptor clustering (Gally et al., 2004). Therefore, we can conclude that the postsynaptic side is 
largely unimpaired in unc-108 n501 and $n 777$ mutants and that the resistance to aldicarb is a clear consequence of reduced acetylcholine release at the neuromuscular junction.
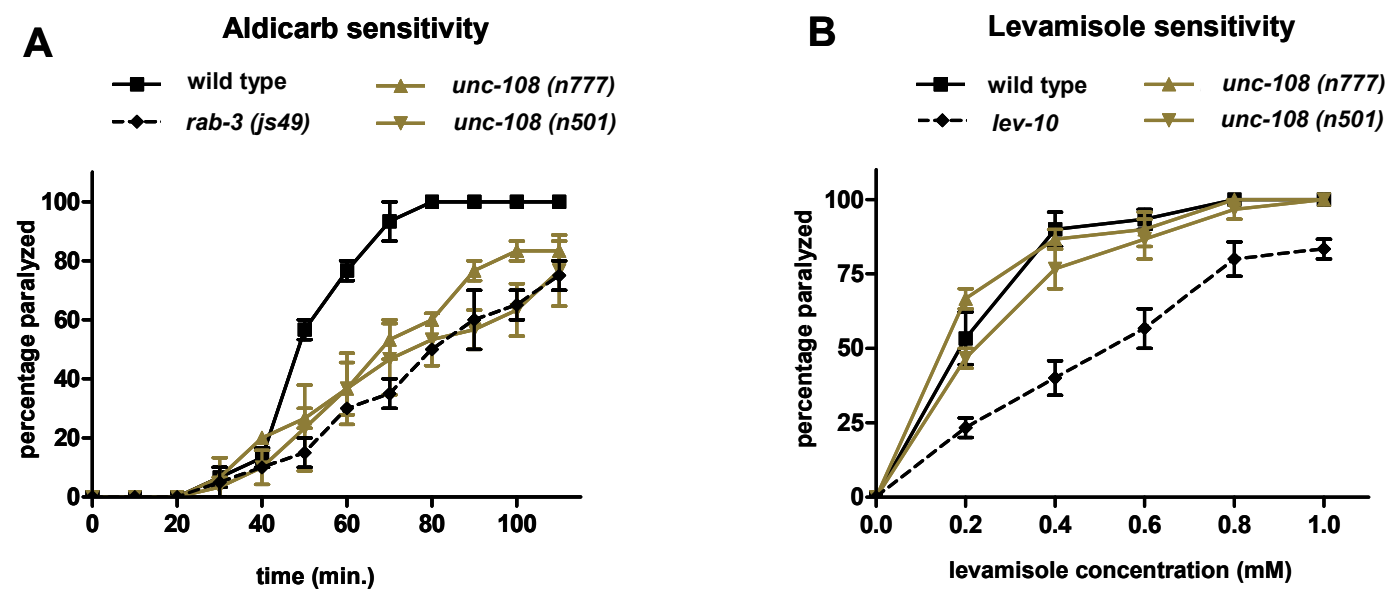

Figure 17. Gain-of-function unc-108 mutants have reduced acetylcholine release, but no defects in the postsynaptic receptor clustering (A) Dominant unc-108 mutants, $n 501$ and $n 777$, are resistant to acetylcholine esterase inhibitor, aldicarb, similarly to rab-3 mutants ( $\mathrm{N}=30$ animals per strain, error bar=SEM).

(B) The postsynaptic side of dominant unc-108 mutants, $n 501$ and $n 777$, shows a wild type like sensitivity to the nicotinic receptor agonist levamisole. lev-10 mutants weakly resistant to levamisole due to nicotinic receptor clustering defects are shown as control $(\mathrm{N}=30$ animals per strain, error bar=SEM).

\subsection{Mutations in UNC-108/RAB-2 affect different neurotransmitter signaling}

Since the aldicarb test is specific for cholinergic signaling, we wanted to see whether other neurotransmitter systems are also affected in unc-108 mutants. Two of these system, GABAergic and serotonergic systems are involved in 
controlling defecation and egg laying, the processes that are stereotyped and fully described in C. elegans (Branicky and Hekimi, 2006; Schafer, 2006; Wolinsky and Way, 1990).
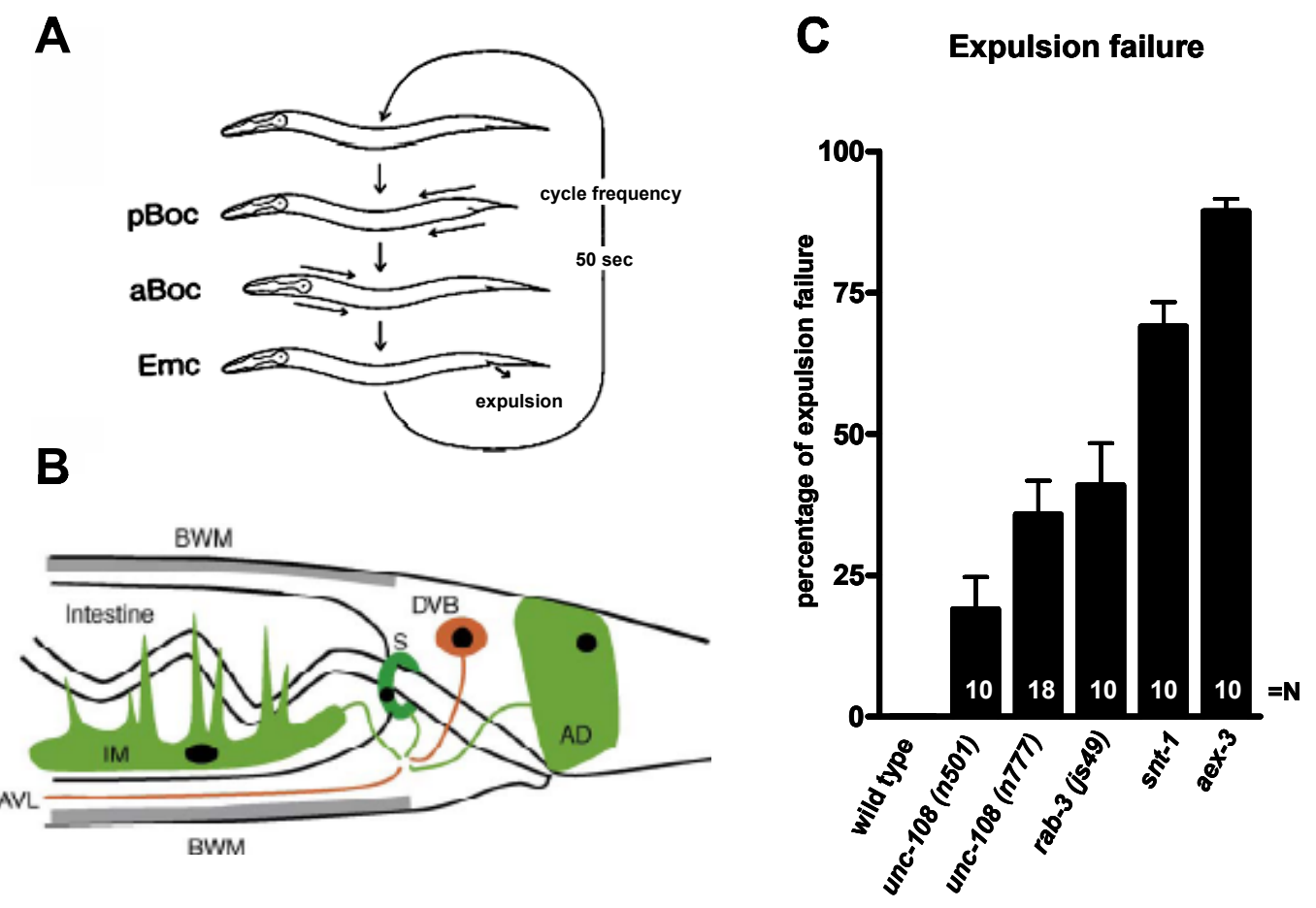

Figure 18. unc-108 mutants have defects in GABAergic signaling.

(A) Defecation cycle is a highly stereotyped process in C. elegans. The cycle starts with the contraction of the posterior side $(\mathrm{pBoc})$ when the intestinal content is pushed towards the head. Then the anterior part of the body contracts pushing food back to the tail. The last step is expulsion. The entire cycle occurs every 50 seconds in wild type. (Jorgensen, 2005)

(B) The defecation cycle is controlled by GABAergic neurons AVL and DVB neurons. The enteric muscle consists of anal depressor (AD), sphincter and two intestinal muscles. The muscles are connected via gap junctions and send muscle arms to the AVL and DVB neurons. The release of GABA leads to contraction of $A D$ and IM (pBoc step) and relaxation of sphincter muscle (expulsion). (Adapted from Jorgensen, 2005)

(C) Each animal was followed for 10 minutes period and the expulsion failures were counted. In unc-108 mutants the expulsion occurs in $60-70 \%$ of the cycles (number of animals analyzed is indicated in the graph bars, error bar=SEM). 
Defecation in $C$. elegans is achieved by cyclical stereotyped motorprogram (Fig. 18A). The first step is the contraction of the posterior side (pBoc), which pushes the intestinal contents towards the head. Then the contraction of the anterior part of the body occurs (aBoc), which pushes the content back to the tail and is followed by expulsion step. The entire program is highly stereotyped and occurs every 50 seconds in wild type. The expulsion step is controlled by GABAergic neurons, which excite and contract the enteric muscles.

While in wild type the expulsion step occurs in every defecation cycle, in unc108 mutants the expulsion step is missing in $30-40 \%$ of the cycles (Fig. 19C). However, the phenotype is not as severe as the one observed in other synaptic proteins, synaptotagmin 1 , snt-1 and aex-3, a RAB-3 and RAB-27 guanine exchange factor (GEF).

One of the best studied behaviors in $C$. elegans is egg-laying. Egg-laying involves a simple motor program involving a small network of motorneurons and specialized muscle cells. One of the key modulators of the egg-laying program is serotonin. Thus, this behavior assay is commonly used for screening for defects in the serotonergic signaling. Serotonin is the activator of hermaphrodite-specific neuron (HSN) which forms synapses with vulva muscles. After fertilization, the eggs remain in uterus for about 2 to 3 hours and are then expelled from the worm through the vulval opening. Most of the eggs laid by worms are still in the blastula stage, continuing with gastrulation and further development in external environment (Fig. 19A). If the signal does not reach the vulva muscle, the eggs are retained in the body cavity, thus 
once the laying event occurs, the embryos are in the later developmental stages (Egl-egg laying defective phenotype).

A
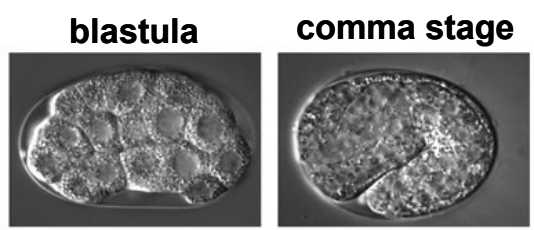

postcomma

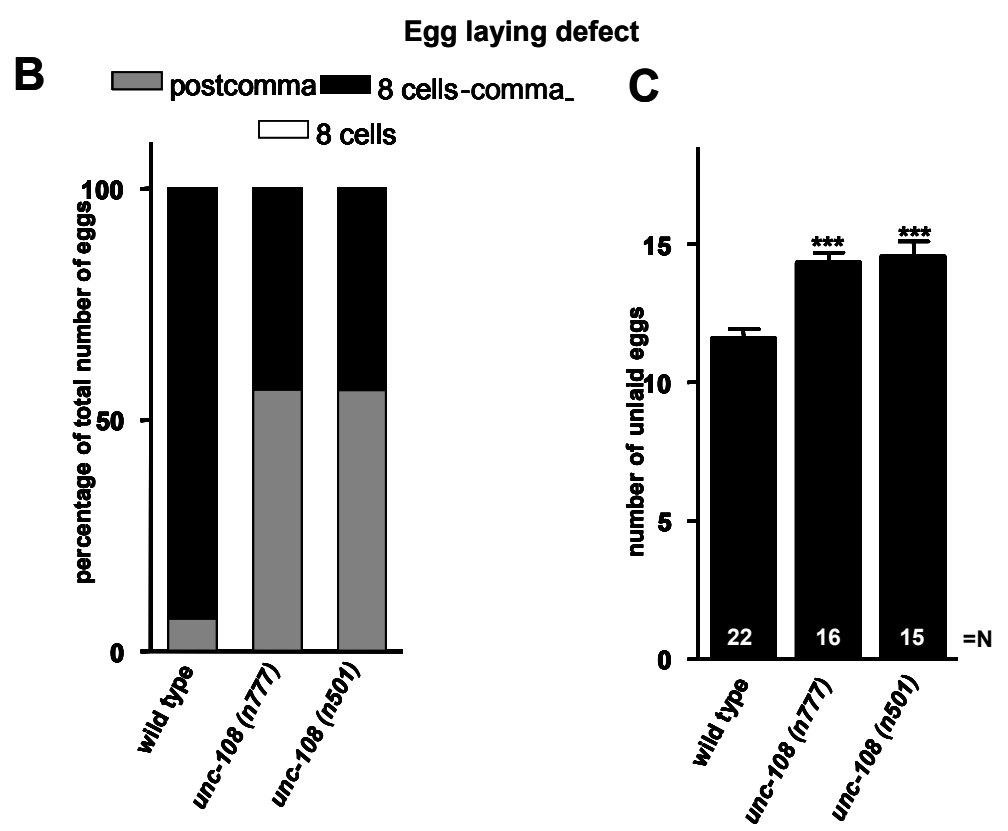

Figure 19. unc-108 mutants have defects in serotonergic signaling

(A) DIC pictures of different embryonic steps in C. elegans development. Blastula stage refers to 8 cell stage, where single embryonic cells are visible. The gastrulation starts with invagination of the syntitium, seen at the microscope as comma stage. Postcomma stage is an advanced gastrula state. (Adapted from Esnard, 2001).

(B) Egg-laying is controlled by serotonergic hermaphrodite specific neuron (HSN). Impairment in serotonergic signaling retains the eggs inside the body and allows the embryos to reach later stages of development before they are laid. In wild type most of the embryos laid are in early gastrula stage (8 cells - comma), while for unc-108 mutants, number of eggs that are in late embryonic development is significantly higher than in wild type (10 animals were analyzed per strain, error bar=SEM).

(C) In adult wild type worms, eggs are retained in the body cavity until the activation of the 
vulva muscles by HSN neuron. Impairment in serotonergic signaling leads to higher retention of eggs. Number of unlaid eggs in unc-108 mutants is higher than in wild type (number of animals analyzed is indicated in the graph bars, error bar is SEM, ${ }^{* *} p<0.005$, Student's Ttest).

In unc-108 mutants the number of laid eggs that are in late gastrulation stage is significantly higher than in the wild type (Fig. 19 B). Accordingly, the number of eggs that are retained in the uterus of unc-108 mutants is higher than in wild type (Fig. 19C). Therefore, we conclude that unc-108 mutants have Egl phenotype and an impairment in serotonergic signaling.

\subsection{Neuronal development is not affected in unc-108/rab-2 mutants}

We wanted to exclude the possibility that the global developmental changes are cause of reduced locomotion and aldicarb resistance in dominant unc-108 alleles. C. elegans has 302 neurons. The number, location and connectivity off all of them is stereotyped and well described. To look at the possible developmental changes of the motorneurons, we crossed unc-108 mutants into strains that have stably GFP expressed specifically in GABAergic motorneurons. The morphology and number as well as the number of comissures that enter dorsal nerve cord are not changed for GABAergic motorneurons in unc-108 mutants when compared to wild type (Fig. 20). 

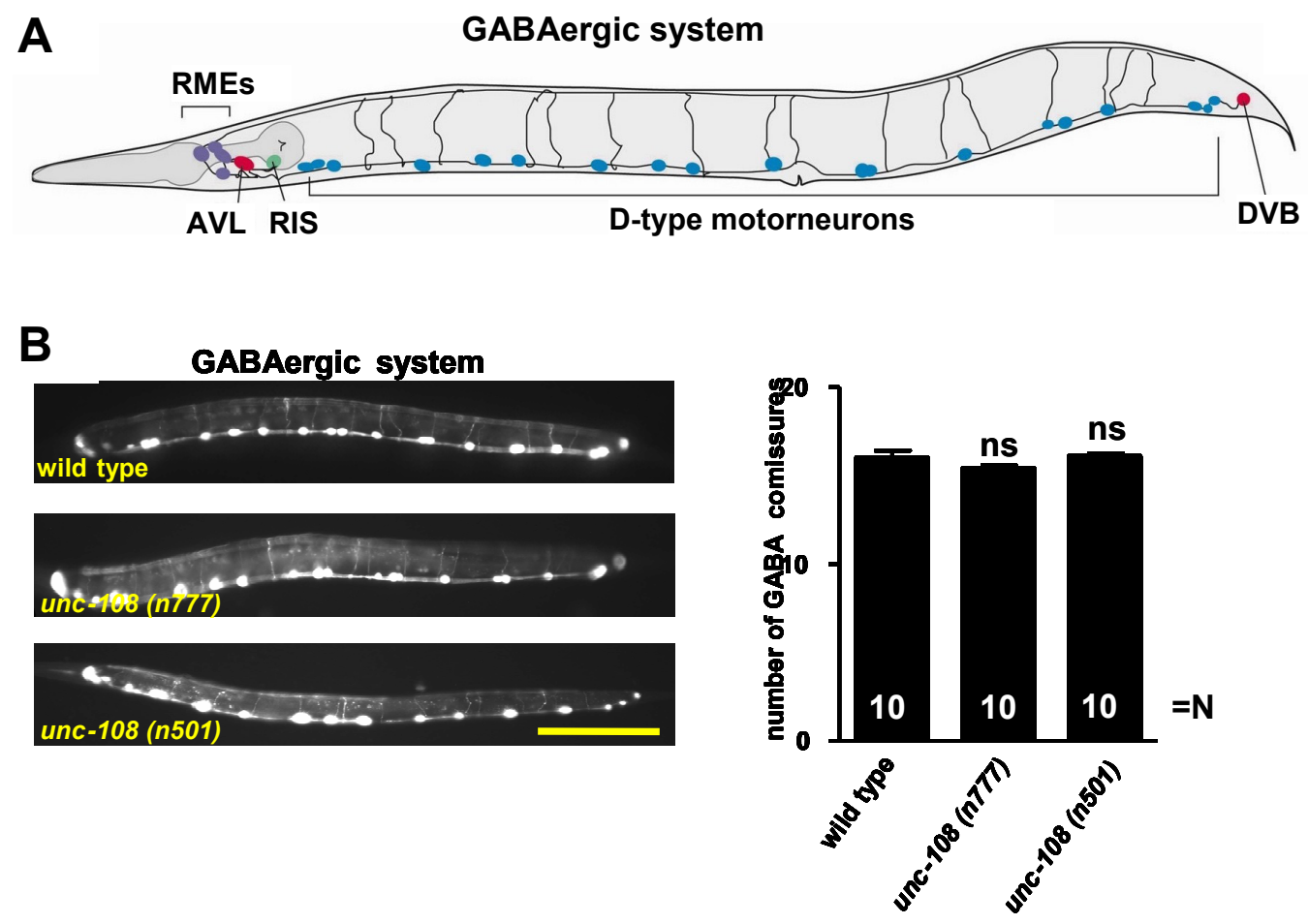

Figure 20. unc-108 have no defects in GABAergic system development

(A) Schematic drawing of 26 GABAergic neurons. D-type motorneurons innervate body wall muscle, RME neurons innervate the head muscles, AVL and DVB innervate the enteric muscles, and RIS is an interneuron. The cell bodies of the GABAergic motorneurons, involved in locomotion control, are located in the ventral nerve cord where from they send the axons into the dorsal nerve cord. (Adapted from Jorgensen, 2005).

(B) GABAergic motorneurons were visualized using a strain expressing GFP under unc-47, a GABA transporter promoter. The development of the GABAergic system appears to be normal in unc-108 mutants as judged by the number of commissures that GABAergic neurons send from ventral to the dorsal side (Scale bar $20 \mu \mathrm{m}$, error bar=SEM, $\mathrm{N}$ is indicated).

To investigate the morphology of the synapses, we looked at the strain stably expressing synaptobrevin tagged to GFP (SNB-1-GFP) in the cholinergic motorneurons. In wild type, SNB-1-GFP shows a puncta staining at the axons of motorneurons (Fig. 21). Each puncta represents a synaptic active zone where the SVs accumulate. The fluorescence intensity corresponds to the 
number of the SV, while the size and the puncta number corresponds to the number of SVs present at the active zones of the synapses and is an indicator of steady state exo-/endocytosis rate. Number, shape and the spacing between the synapses of the cholinergic motorneurons projecting their axons into dorsal nerve cord is not changed in unc-108 mutants, as judged by the quantitative measurements of fluorescently labeled SV markers, snb-1 (Fig. 21). We also found that the intensity of SNB-1-GFP staining is similar to wild type in unc-108 mutants (Fig. 21). This suggests that the morphology of synapses, as well as SV recycling is not changed in unc-108 mutants.
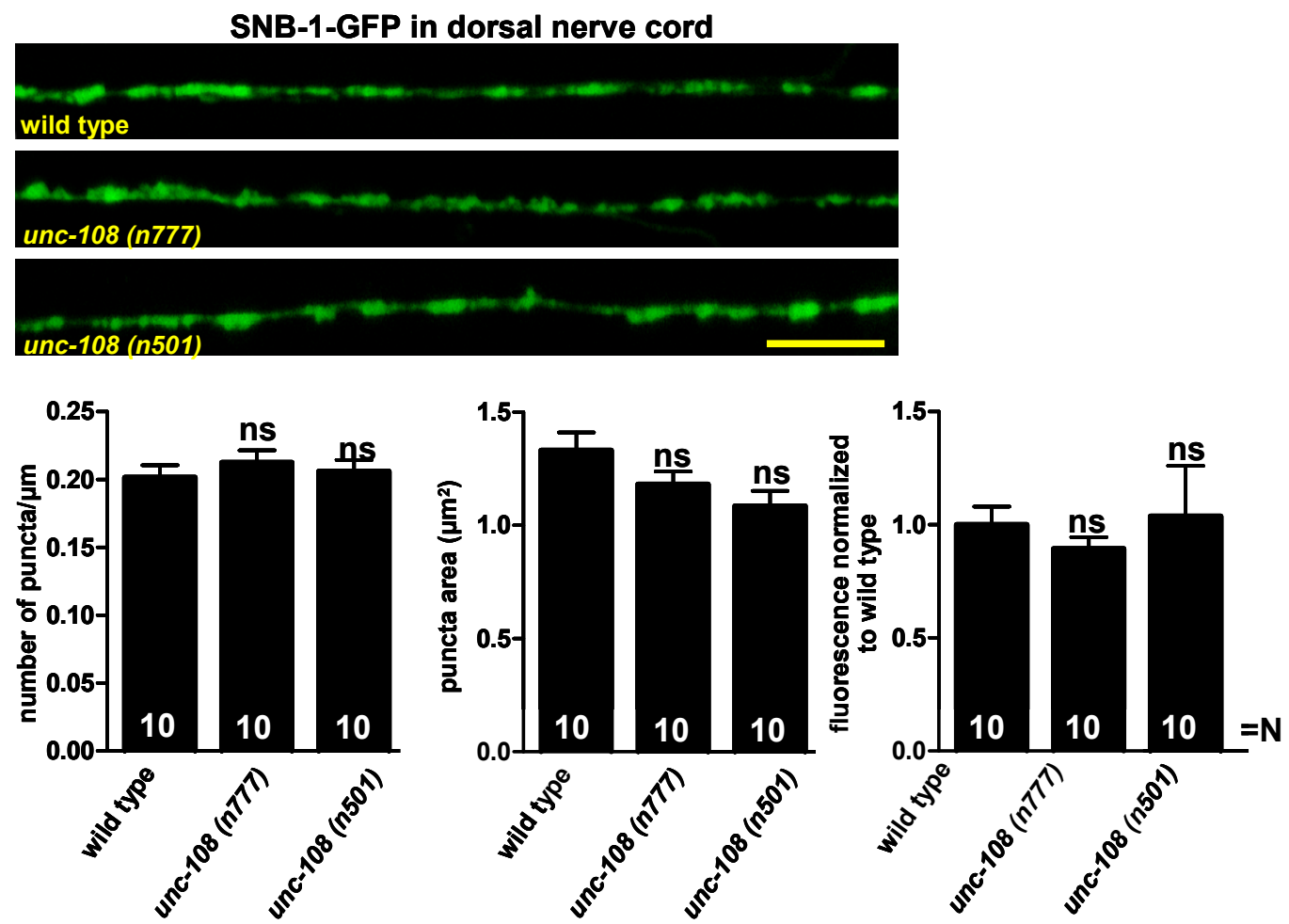

Figure 21. unc-108 mutants have no defects in synaptic morphology

Animals expressing SNB-1-GFP in cholinergic motorneurons were crossed into unc-108 background. The synapse morphology appears to be similar between wild type and unc-108 mutants, as judged by SNB-1-GFP puncta number, size and average fluorescence (Number of animals analyzed is indicated in the graph bars, scale bar is $10 \mu \mathrm{m}$, error bar=SEM). 
To completely rule out the possibility of synaptic morphological changes causing the defect in neurotransmission, we looked at the ultrastructural level of synapses of unc-108 and wild type animals by high pressure freeze (HPF) electron microscopy (EM).

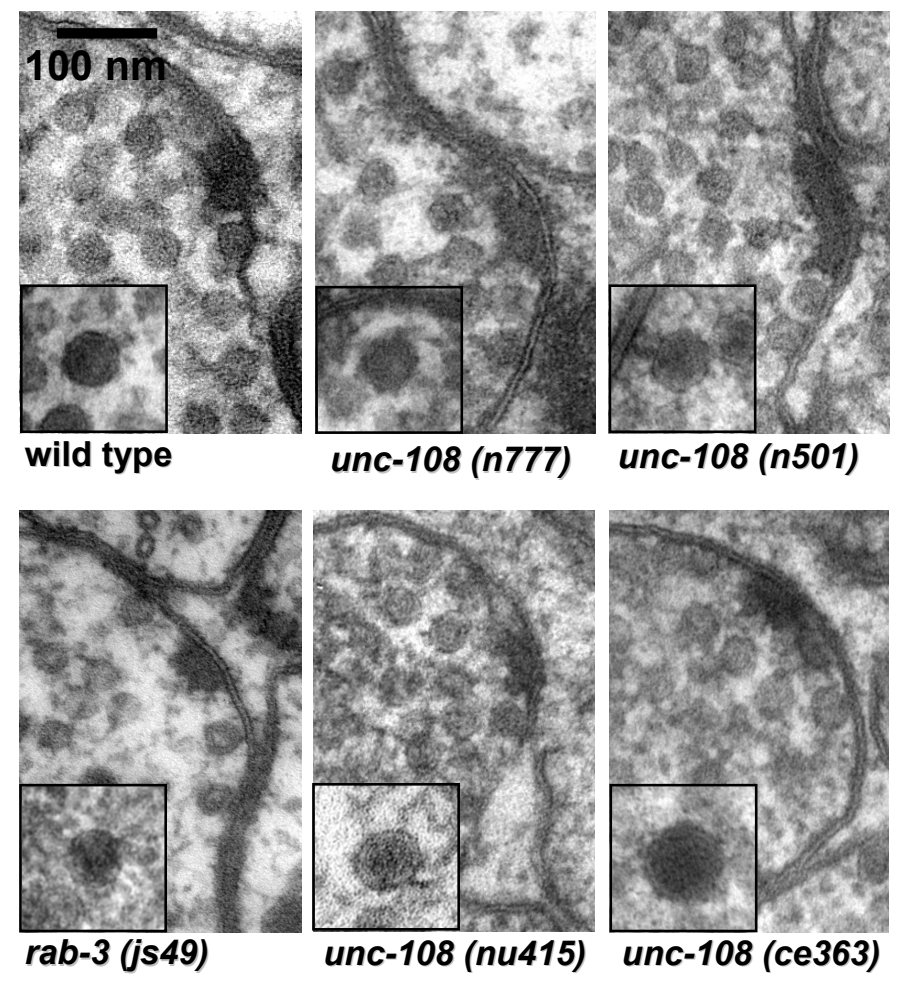

Figure 22. The morphology of the synapse is not changed in unc-108 mutants.

The morphology of motorneuron synapses in the dorsal nerve cord in different $C$. elegans strains are shown in $40 \mathrm{~nm}$ HPF EM cross sections, with electron micrographs of single DCV shown in inlet. (Courtesy of Jan Hegermann)

As shown in panel (Fig. 22) the morphology of the synapse, presynaptic density as well as the number and size of SVs appears normal in unc-108 animals. The impairment in neurotransmission as well as aldicarb resistance can be caused by changes in distribution of SVs, which is the case for mutants for rab-3, a protein involved in SV tethering. HPF EM shows that the 
distribution of SVs is similar to wild type and clearly distinguishable from rab-3 mutants which have reduced number of SVs close to the presynaptic density (Fig. 23). Furthermore, the number and size of SVs in unc-108 mutants is similar to wild type (Table 5). Therefore, we conclude that the synaptic development and morphology is not affected in unc-108 mutants.

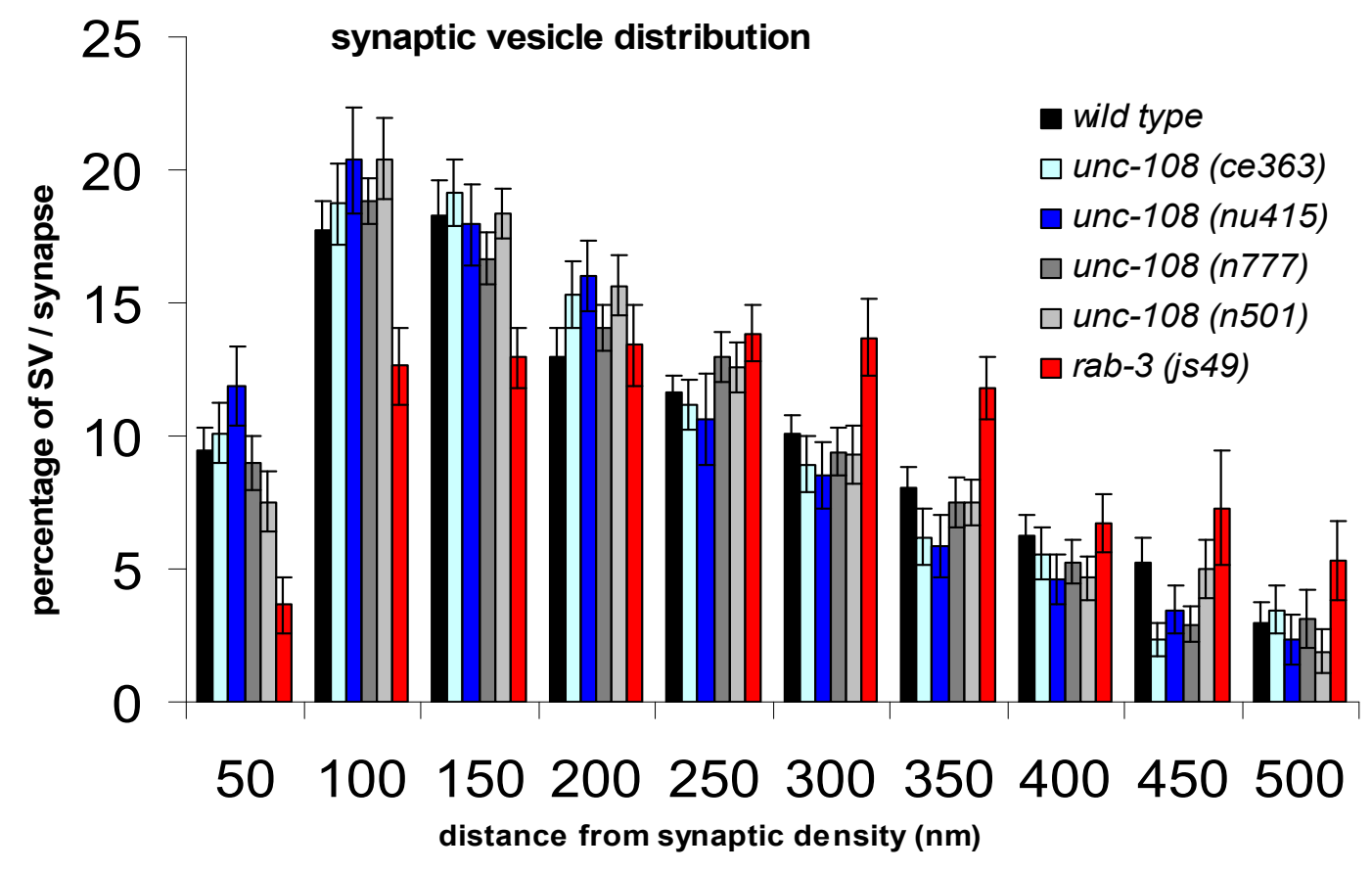

Figure 23. The number and the distribution of SVs is not changed in unc-108 mutants.

The SV distribution relative to the presynaptic density at release sites was analyzed for unc108. SV distribution in rab-3 mutants, exhibiting SV tethering defects, is shown as control. unc-108 data is not significantly different compared to wild type (one way ANOVA with Dunett posttest). (Courtesy of Jan Hegermann and Christian Olendrowitz) 


\begin{tabular}{lcccc}
\hline Genotype & $\begin{array}{c}\text { Number of } \\
\text { profiles/animals } \\
\text { analyzed }\end{array}$ & $\begin{array}{c}\text { Average } \\
\text { synaptic } \\
\text { area }\left(\mu \mathrm{m}^{2}\right)\end{array}$ & $\begin{array}{c}\text { SVI } \\
\text { profile }\end{array}$ & $\begin{array}{c}\text { Average diameter } \\
\text { of SVs }(\mathrm{nm})\end{array}$ \\
\hline wild type & $30 / 5$ & $0.25 \pm 0.02$ & $34.9 \pm 2.4$ & $30,3 \pm 0.7$ \\
unc-108 (n777) & $34 / 4$ & $0.20 \pm 0.01$ & $39.3 \pm 1.8$ & $28,4 \pm 0.7$ \\
unc-108 (n501) & $31 / 4$ & $0.23 \pm 0.03$ & $38.7 \pm 1.7$ & $27,7 \pm 0.5$ \\
unc-108 (ce363) & $28 / 3$ & $0.25 \pm 0.02$ & $35.1 \pm 2.8$ & $30,3 \pm 0.7$ \\
unc-108 (nu415) & $26 / 4$ & $0.21 \pm 0.01$ & $29.2 \pm 1.5$ & $26,6 \pm 0.6$ \\
rab-3 (js49) & $13 / 3$ & $0.22 \pm 0.02$ & $37.7 \pm 3.4$ & $27,8 \pm 0.6$ \\
\hline
\end{tabular}

Table 5. SV statistics for unc-108 mutants.

Statistics on SV numbers and diameters as determined by HPF EM, the average synaptic area, number of synapses and independent animals analyzed are indicated (Courtesy of Jan Hegermann and Christian Olendrowitz)

\section{5 unc-108/rab-2 mutants have normal SV release}

It has been shown that RNAi treatment that leads to reduced acetylcholine release causes aldicarb resistance (Sieburth et al., 2005). Also, the response to aldicarb can be controlled by neuropeptides (Jacob and Kaplan, 2003; Sieburth et al., 2005). To distinguish between these two possibilities we recorded the currents in the body wall muscle evoked by electrically stimulating the nerve cord. The average amplitude of evoked responses was not significantly different from wild type in either $n 501$ or $n 777$ alleles (Fig. 24) unlike rab-3, which have been shown to have reduced evoked response (Gracheva et al., 2008). 

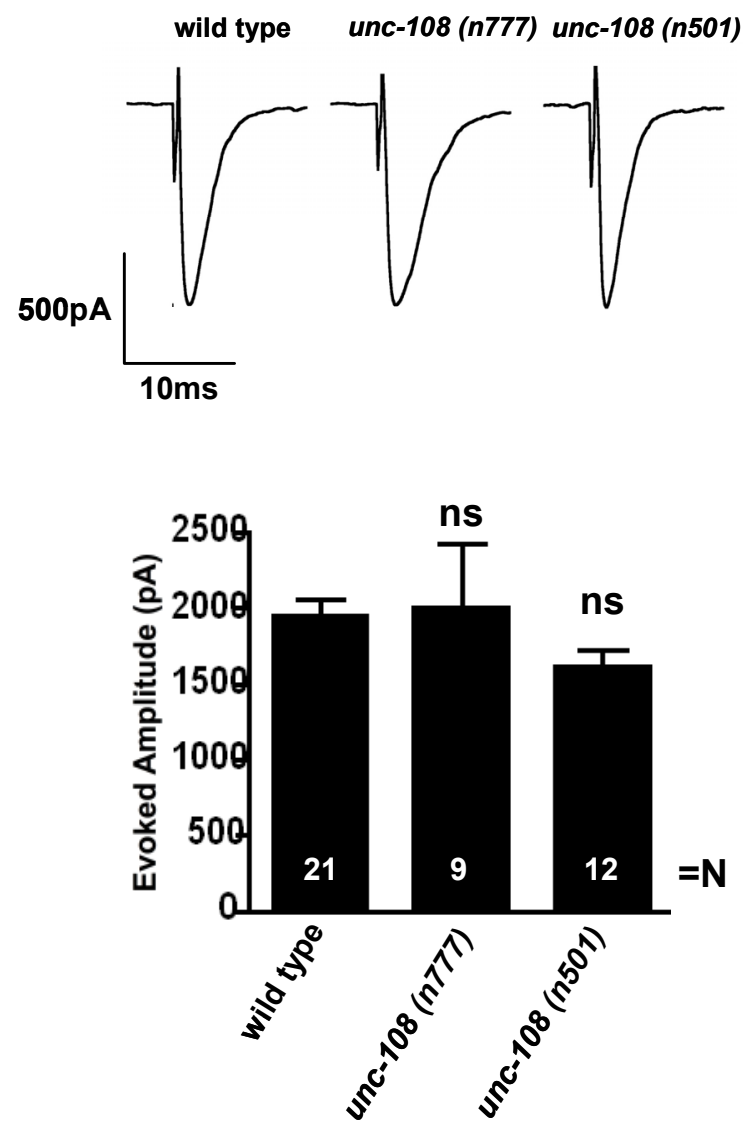

Figure 24. unc-108 mutants have no defects in SV release at the neuromuscular junction.

The evoked release of SVs at the neuromuscular junction is similar between the wild type and dominant unc-108 mutants. (Number of animals analyzed is indicated in the graph bars, error bar is SEM). (Courtesy of Janet Richmond).

\subsection{Increase in diacylglycerol levels rescues}

neurotransmission defects in unc-108 mutants to wild type level

Phorbol esters and diacylglycerol (DAG) promote the synaptic and dense core vesicle exocytosis in neurons through binding to $\mathrm{C} 1$ domain containing proteins and promoting their association with the plasma membrane (Silinsky and Searl, 2003). In C. elegans phorbol esters induce the hypersensitivity to aldicarb (Nurrish et al., 1999, Miller et al., 1999). It has bees been shown that 
proteins involved in synaptic and DCV priming and fusion regulate the responsiveness to phorbol esters. Mutations in rab-3, unc-13, pkc-1 and unc31/CAPS lead to phorbol ester resistance (Sieburth et al., 2005; Sieburth et al., 2007) (Fia. 25).

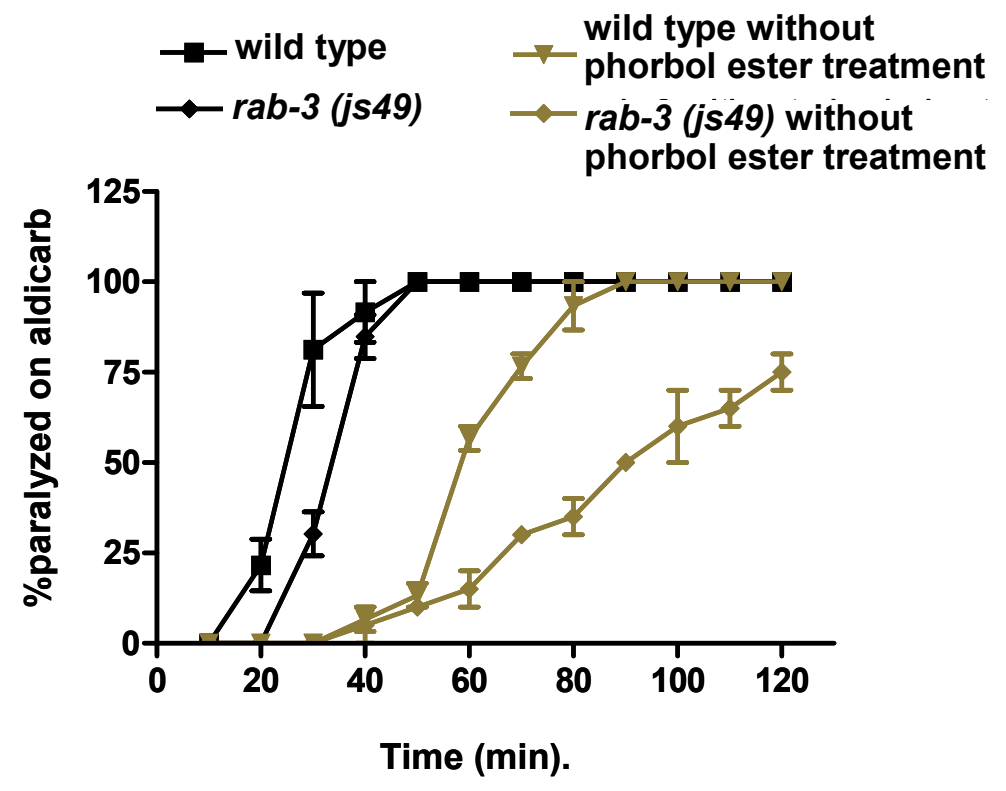

Figure 25. rab-3 mutants are resistant phorbol esters.

Phorbol esters are orthologues of diacylglycerol. Exposure to phorbol esters induces hypersensitivity to aldicarb in wild type animals. In rab-3 mutants, who are defective in SV tethering, the phorbol esters induce only partial hypersensitivity to aldicarb. $(\mathrm{N}=30$ animals per strain, error bar is SEM).

However, the mutants for proteins inducing the DAG signaling cascade, once exposed to phorbol esters manage to restore the wild type levels of acetylcholine secretion (Nurrish et al., 1999). To test whether the impairment in modulation of synaptic transmission is responsible for the uncoordinated phenotype of unc-108 mutants, we examined the effects of phorbol esters. We found that even though the $n 501$ allele was partially resistant to phorbol ester, the acetylcholine release in $n 777$ allele was brought up to the wild type levels (Fig. 26). 


\section{phorbol ester sensitivity}

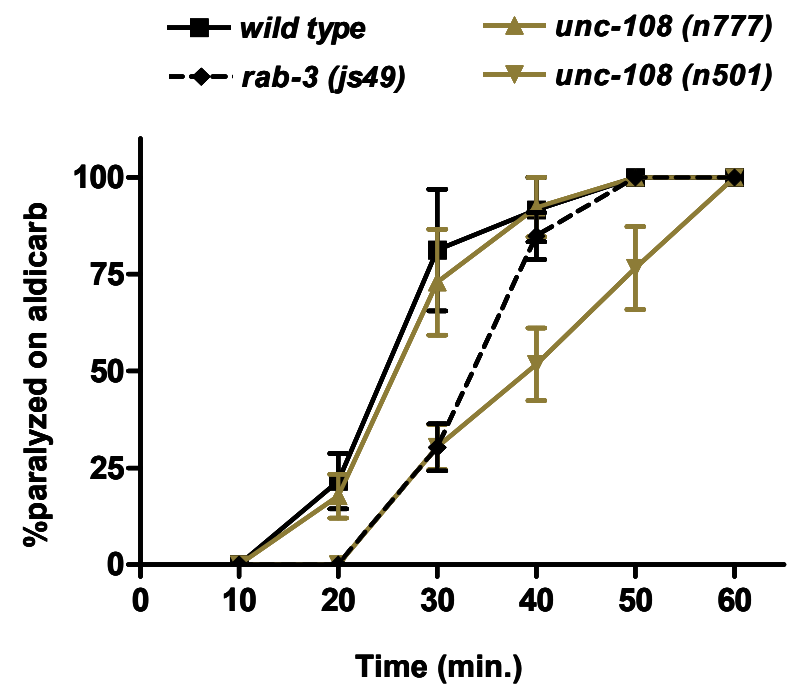

Figure 26. The exposure of unc-108 mutants to phorbol esters brings back the responsiveness to aldicarb to wild type levels in unc-108 (n777) allele.

Worms were exposed to phorbol esters that act as an ortholog of diacylglycerol. This leads to increased acetylcholine at the neuromuscular junction which is in the case of $n 777$ allele the same level as for wild type ( $N=30$ animals per strain, error bar is $\mathrm{SEM}$ ).

To further test this we overexpressed the gain-of-function mutant of excitatory Gaq subunit (EGL-30), which has been shown to stimulate the production of diacylglycerol at the presynaptic terminal (Nurrish et al., 1999). The overexpression increases the locomotion rate in unc-108 ( $n 777)$ allele. The similar amelioration of the locomotory phenotype was observed upon overexpression of myrostilated UNC-13 (myr-unc-13), which mimics the membrane recruited and activated UNC-13 protein (Fig. 27). 

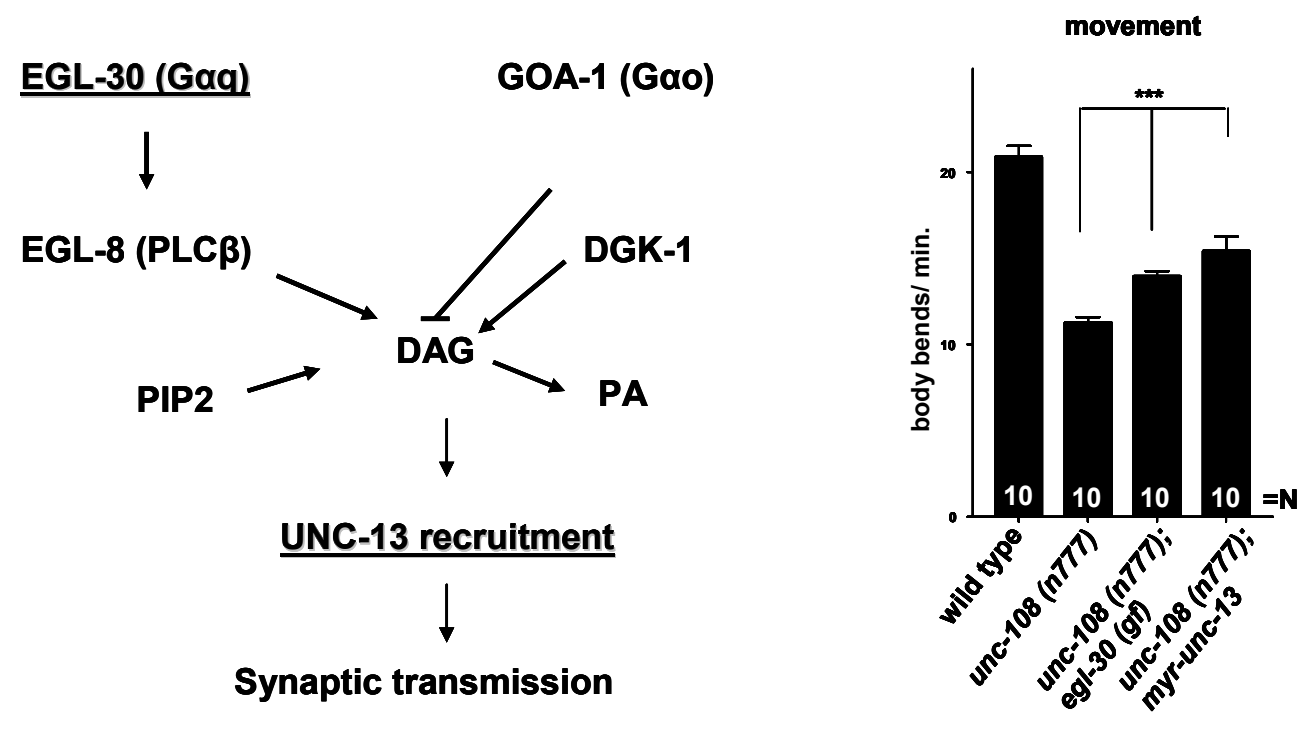

Figure 27. The overexpression of gain-of-function EGL-30 or active, myrostilated UNC13 increase the movement in unc-108 (n777).

The gain-of-function mutant of Gaq subunit EGL-30 was overexpressed in unc-108 (n777). The overexpression of EGL-30 (gf) stimulates the signaling cascade in the cell which stimulates the conversion of phosphoinositole-4,5-biphosphate into diacylglycerol. Diacylglycerol binds to $\mathrm{C} 1$ domain of UNC-13, thus stimulating the SV priming and fusion. The overexpression of the activated, myrostilated UNC-13 also leads to increase in movement in unc-108 (n777). (Number of animals analyzed is indicated in the graph bars, error bar is SEM, ${ }^{* * *} \mathrm{p}<0.005$ Student's T-test).

Furthermore, by generating a double mutant between unc-108 and diacylglycerol kinase (dgk-1) mutants, the enzyme responsible for converting DAG to phosphatidic acid (PA), we rescued the movement phenotype observed in unc-108 mutants (Fig. 28). dgk-1 mutants are hyperactive: they have higher movement activity when compared to wild type and are hypersensitive to aldicarb. However, in unc-108 background, the movement in dgk-1 mutants is comparable to wild type levels. In support to these observations, unc-108 was shown to also suppress the hyperactivity of goa-1 
mutants, a Gao subunit involved in attenuating the DAG signaling cascade (Edwards et al., in press). These results suggest that in unc-108 mutants the modulation of synaptic activity at the presynaptic site is impaired.
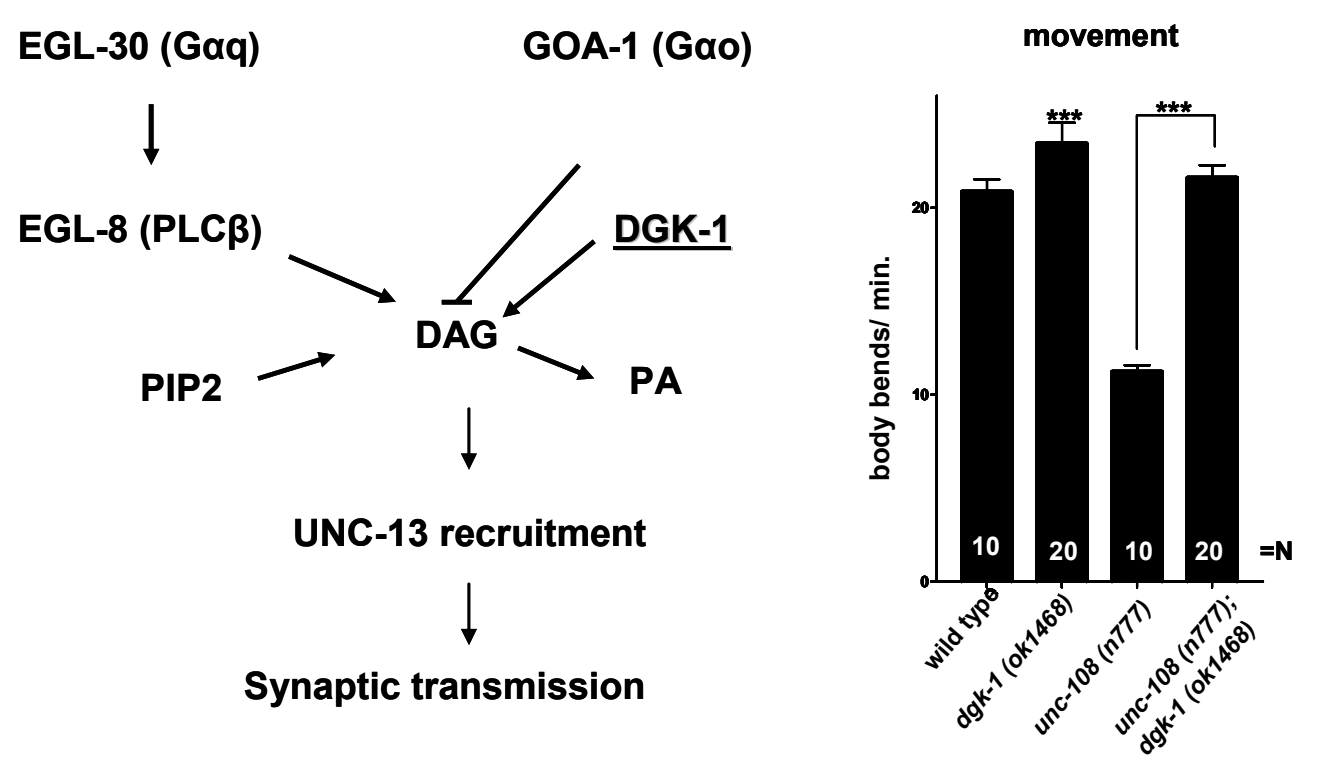

Figure 28. Deletion of diacylglycerol kinase (dgk-1) rescues the movement defect in unc-108 mutants.

unc-108 mutants were crossed into dgk-1 deletion mutants. DGK-1 is responsible for converting diacylglycerol into phosphatidic acid and thus reducing the amount of diacylglycerol at the synapse. By eliminating the DGK-1, the turnover of diacylglycerol is reduced and therefore the diacylglycerol levels at the presynaptic terminal are constantly higher. $d g k-1$ mutants are hyperactive and show increased movement when compared to wild type. The deletion of $d g k-1$ in unc-108 mutants rescues the movement phenotype. (Number of animals analyzed is indicated in the graph bars, error bar is SEM, ${ }^{* *} \mathrm{p}<0.005$, Student's Ttest).

Diacylglycerol results as a product of cleavage of phosphatidylinositol-4,5biphosphate $\left(\mathrm{PI}(4,5) \mathrm{P}_{2}\right)$ into DAG and inositol-1,4,5-triphosphate (IP3), a reaction which is catalyzed by phospholipase $C$ (EGL-8). One possibility was that altered UNC-108/RAB-2 activity results in a reduction of $\mathrm{PI}(4,5) \mathrm{P}_{2}$ levels 
at the plasma membrane and therefore reduced DAG levels. To further investigate this, we made a construct containing a $\mathrm{PH}$ domain of phospholipase $\mathrm{C} \delta$ (PH-PLCס) tagged to GFP and expressed under rab-3 promoter (Marza et al., 2008). It has been shown previously that $\mathrm{PH}$ domain
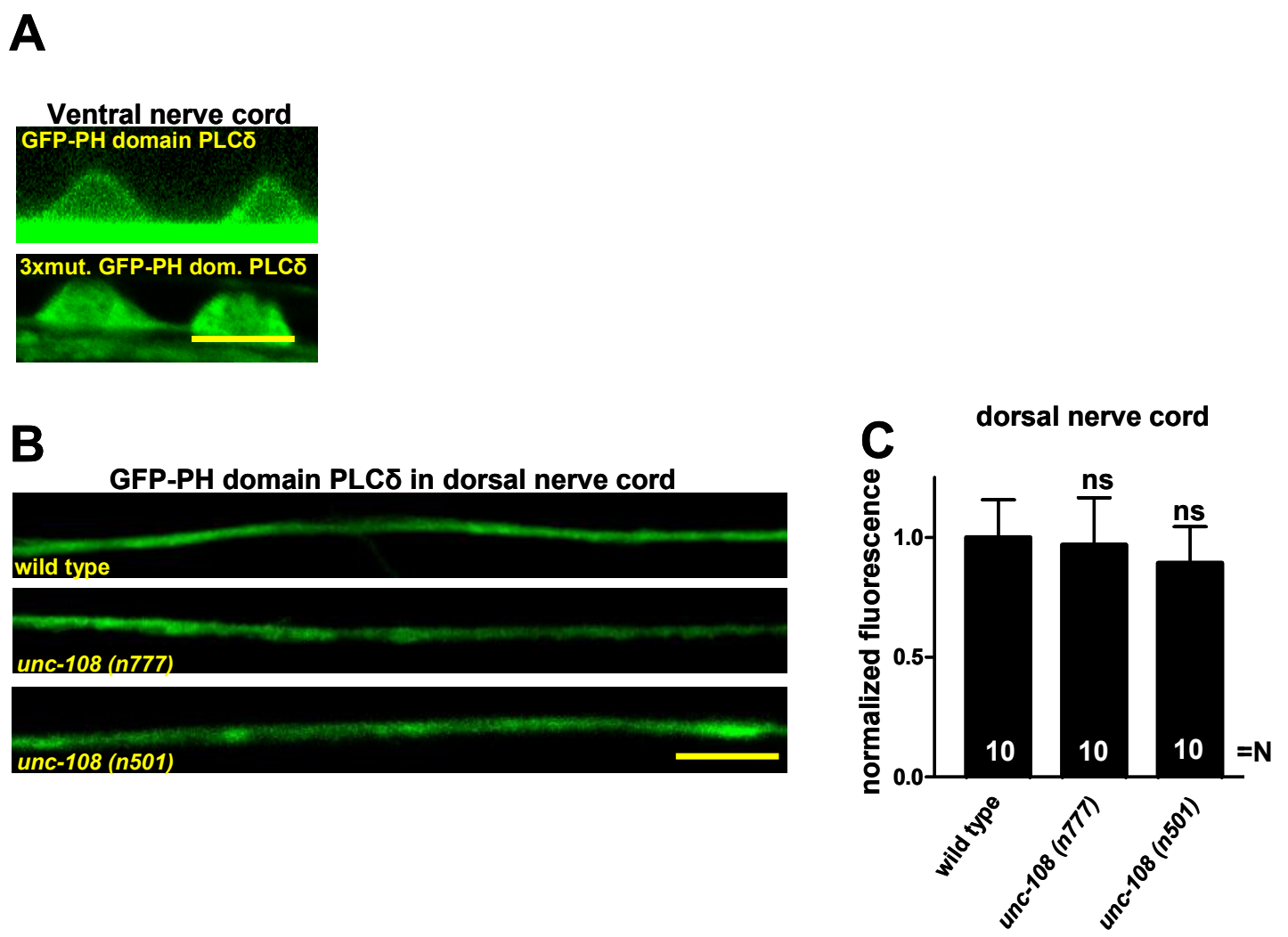

Figure 29. The $\mathrm{PI}(4,5) \mathrm{P}_{2}$ levels are not changed in unc-108 mutants

(A) Wild type form of GFP tagged PH domain of PLCס localizes to the plasma membrane of neuronal soma in the ventral nerve cord. Triple mutant of PLCס PH domain (K30A, K32A, $\mathrm{W} 36 \mathrm{~N})$, deficient in $\mathrm{PI}(4,5) \mathrm{P}_{2}$ binding is retrieved from the plasma membrane and is mainly cytoplasmatic. Scale bar is $5 \mu \mathrm{m}$.

(B) Left - Representative images of GFP-PH domain of PLC $\delta$ expression in the dorsal nerve cord of wild type and unc-108 mutants. The GFP fluorescence was quantified and normalized to wild type (shown on the right). $\mathrm{PI}(4,5) \mathrm{P}_{2}$ levels in unc-108 mutants appear to be the same as in wild type animals. Scale bar is $10 \mu \mathrm{m}$. (Error bars are SEM, N=10 animals per strain). 
of PLCס binds to $\mathrm{PIP}_{2}$ selectively (Milosevic et al., 2005; Stauffer et al., 1998; Varnai and Balla, 1998). We observed a strong GFP labeling of the plasma membrane. As shown in figure 29A, the mutations in amino acids responsible for $\mathrm{PIP}_{2}$ binding lead to accumulation of PH-PLCס-GFP in the cytoplasm, proving the binding specificity (Milosevic et al., 2005). unc-108 mutants have no changes in $\mathrm{PIP}_{2}$ amounts at the synapses. From these data, we conclude that the uncoordinated phenotype and aldicarb responsiveness observed in unc-108 mutants are induced by changes in the DAG signaling at the presynaptic terminal and the impairment is probably occurring early in the signaling cascade.

\subsection{UNC-108/RAB-2 acts in DCV signaling pathway}

Neuropeptides have crucial role in modulating the transmission at the neuromuscular junction. Neuropeptides are packaged into DCVs and are coreleased with SVs in axons. It has been previously shown that DCV signaling also regulate aldicarb responsiveness (Jacob and Kaplan, 2003; Sieburth et al., 2005). Inactivation of proneuropeptide proteases, egl-3 and egl-21, as well as the DCV priming factor unc-31/CAPS, all cause aldicarb resistance. Furthermore, unc-31/CAPS mutants have severe locomotion defects. 

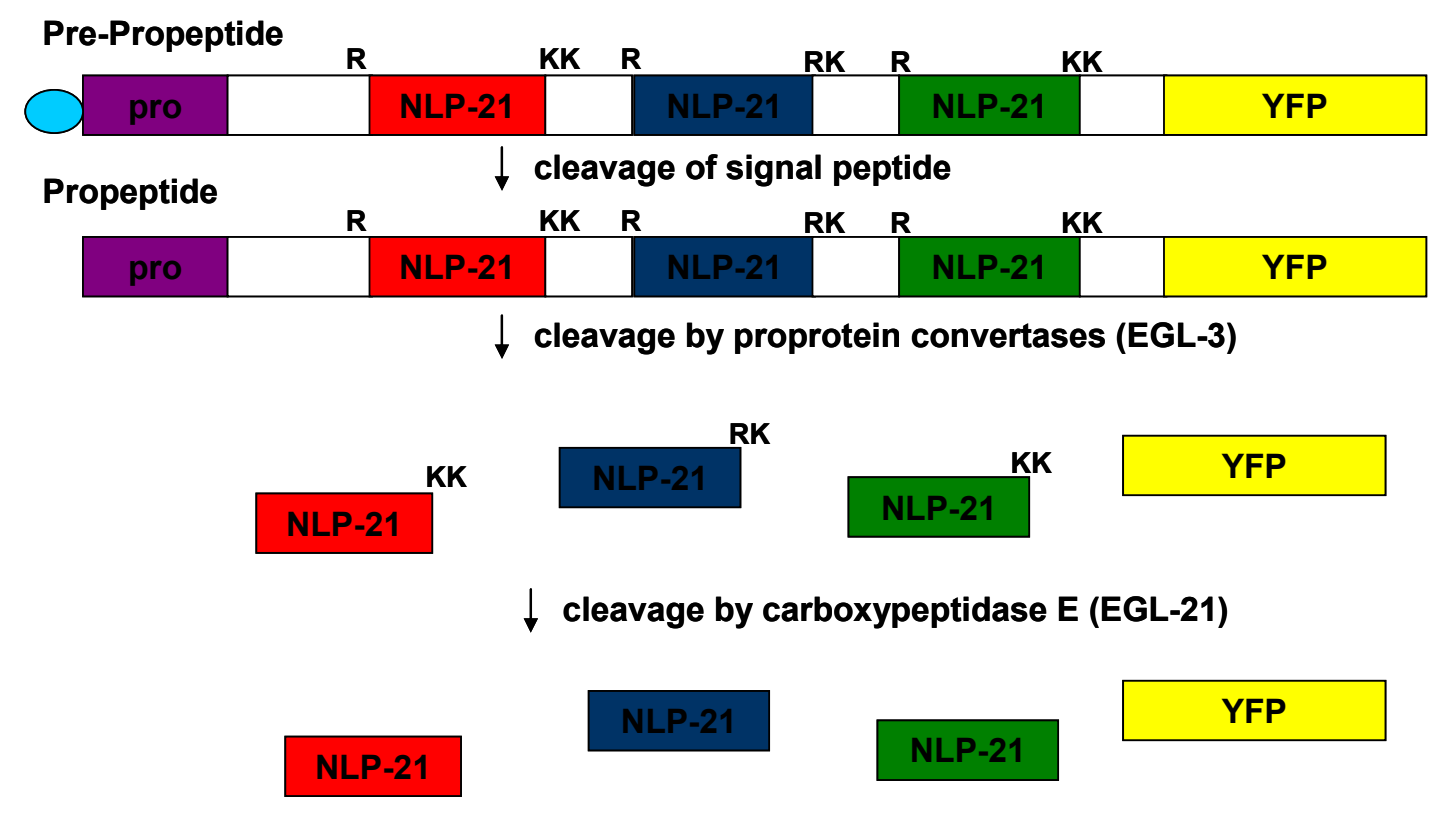

Figure 30. Processing of NLP-21-YFP.

Pre-pro-NLP-21-YFP is cleaved by signal peptidase in the endoplasmic reticulum to release the signal peptide. The propeptide pro-NLP-21-YFP is further cleaved C-terminally to mono-, di-, or tribasic residues (indicated by $\mathrm{K}$ and $\mathrm{R}$ ) by proprotein convertases, such as EGL3/PC2, releasing the YFP. The basic amino acids are removed by carboxypeptidases E, such as EGL-21, to yield the basic neuropeptides.

To test whether DCV signaling is defective in unc-108 mutants, we used a strain previously described by Sieburth et al. (Sieburth et al., 2007). The nuls183 strain has integrated and stably expressed neuropeptide precursor NLP-21 fused to YFP (Fig. 30) in the DA motorneurons. DA motorneurons are cholinergic motorneurons, that form neuromuscular junctions at the dorsal side (dorsal nerve cord) and receive synaptic input on the ventral nerve cord (Fig. 31A). Therefore, dorsal nerve cord is considered as presynaptic side, while the ventral nerve cord is postsynaptic. 
A

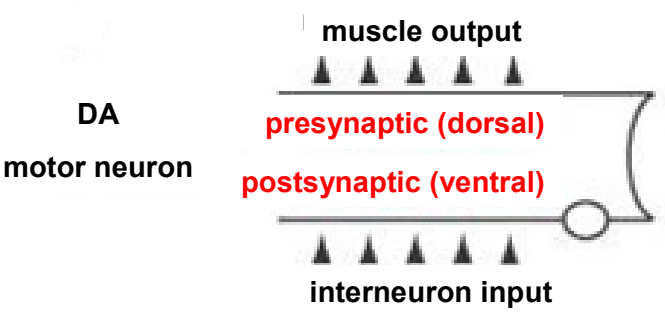

B

\section{Cholinergic motor neuron}

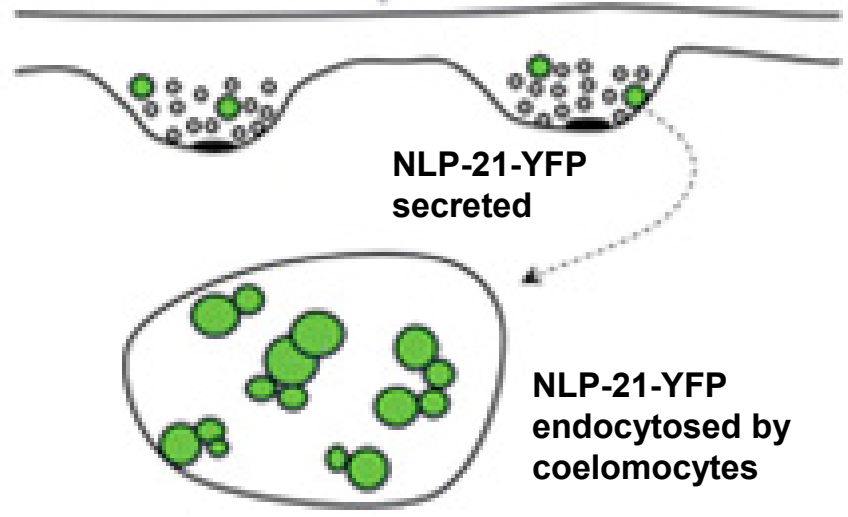

Figure 31. NLP-21-YFP is packaged into DCVs and released at the synapses

(A) NLP-21-YFP is expressed under unc-129 promoter, which drives expression specifically in the DA type cholinergic motorneurons. The cell bodies of DA motorneurons are located in ventral nerve cord and send axonal projections to the dorsal nerve cord forming synapses with body wall muscles (Adapted from Sieburth et al., 2006).

(B) In the nuls183 strain stably expressing NLP-21-YFP in the cholinergic motorneurons, the neuropeptide is packaged into the DCVs and secreted at the synapse. Once at the synapse, the neuropeptide defuses into the coelom, where it is taken up by scavenger cells coelomocytes via pinocytosis. Therefore, the YFP fluorescence at the dorsal nerve cord represents the relative amount of the neuropeptide present at the synapses, while the YFP fluorescence in the coelomocytes corresponds to the amount of neuropeptide secreted at the synapse. (Adapted from Gracheva et al., 2007).

It has been shown that the fusion protein is packaged into DCVs and released at the neuromuscular junction. Therefore, by quantifying the fluorescence of 
the fusion protein at the axons projecting to the dorsal nerve cord, one can relatively quantify the amount of the neuropeptides present at the synapses (Fig. 31B). We found a significant decrease in NLP-21 derived YFP fluorescence at the synapses in the dorsal nerve cord of unc-108 animals. The NLP-21 derived YFP levels were decreased by $70 \%$ as compared to wild type levels in both gain- and loss- of function mutants (Fig. 32). However, the number of NLP-21-YFP puncta in the dorsal nerve cord is not changed compared to wild type, suggesting that number of DCV release sites is similar in unc-108 compared to wild type (Fig. 32).

A

NLP-21-YFP in dorsal nerve cord

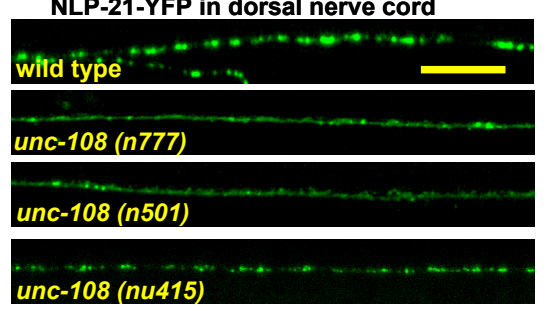

B

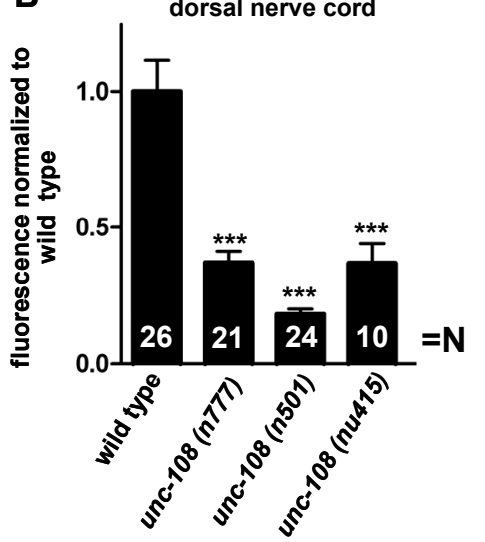

C dorsal nerve cord

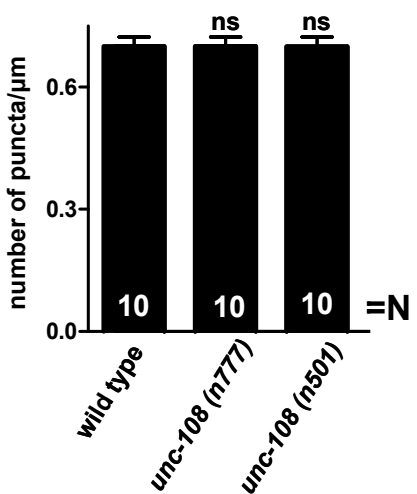

Figure 32. unc-108 mutants have strongly reduced soluble cargo in DCVs at the synapses.

Representative images of YFP tagged NLP-21 neuropeptide in dorsal nerve cord of wild type and unc-108 mutants. Puncta fluorescence was quantified and normalized to the wild type. unc-108 mutants show strong reduction in neuropeptide abundance in the axons of cholinergic motorneurons. Number of puncta per $\mu \mathrm{m}$ of dorsal nerve cord was also quantified. Number of NLP-21-YFP puncta appears to be similar in wild type and unc-108 mutants. Scale bar $10 \mu \mathrm{m}$. (Number of animals analyzed is indicated in the graph bars, error bar is SEM, ${ }^{* * *} p<0.005$, Student's T-test). 
Once released at the synapse, NLP-21 derived YFP diffuses from synaptic cleft into the coelom and is taken up by six scavenger cells, called coelomocytes (Fig. 31B). Thus the amount of the fluorescence endocytosed by coelomocytes directly corresponds to the amount of the peptide released at the synapse if the endocytosis in coelomocytes is not affected. To look at the coelomocyte endocytosis, we used a strain that expresses GFP in the body wall muscle, with a tagged sequence that leads to constitutive secretion of the fluorescent protein into the coelom. Once secreted into the pseudocoelomic space, GFP is taken up by the six coelomocytes by pinocytosis (Fig. 33A). In the unc-108 mutants, the amount of GFP in coelomocytes is similar relative to wild type levels (Fig. 33B and C). To exclude any possibility in defective endocytotic pathway in unc-108 mutants, we also looked at the sizes and numbers of compartments that stain positively to early and late endosomal marker (RME-8) and endosomal/lysosomal marker (CUP-5) (Fig. 34A). In gain-of-function alleles, $n 501$ and $n 777$, the size of the vesicular structures in the endocytotic pathway remains the same, unlike the loss- of function, ce365 allele, which has changes in endo-lysosomal trafficking (Fig. 34B, Chun et al., 2008). Therefore, we assume that the decrease of $70 \%$ of NLP-21-YFP fluorescence in unc-108 coelomocytes is a clear read out of the reduced neuropeptide secretion from the synapses and directly corresponds to the decreased amount of peptide present at the synapse (Fig. 35). 


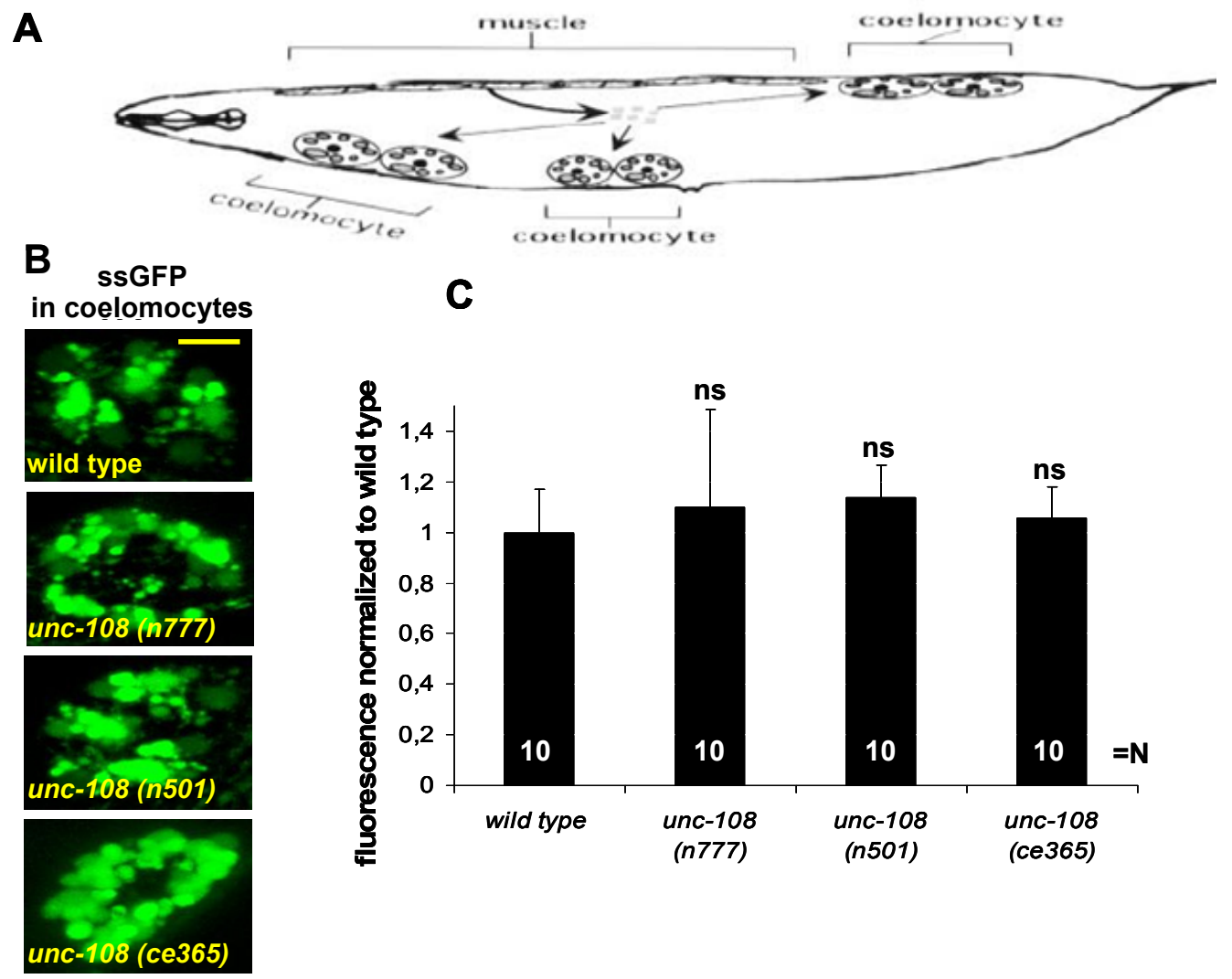

Figure 33. The fluid phase uptake is not impaired in unc-108 coelomocytes.

(A) The strain used to look at the fluid phase uptake in coelomocytes has GFP tagged to the sequence that makes the protein enter the constitutive secretory pathway in body wall muscles. Once secreted from the body wall muscle into the coelom, GFP is taken up by 6 scavenger cells, coelomocytes. The coelomocyte GFP fluorescent is a relative measure of the pinocytosis rates. (Adapted from Fares and Greenwald, 2001).

(B) The representative images of wild type and unc-108 coelomocytes. Scale bar is $2 \mu \mathrm{m}$.

(C) The GFP fluorescent was quantified and normalized to wild type. The GFP uptake in unc108 mutants shows no significant deference when compared to wild type (Number of animals analyzed is indicated in graph bars, error bar is SEM). 

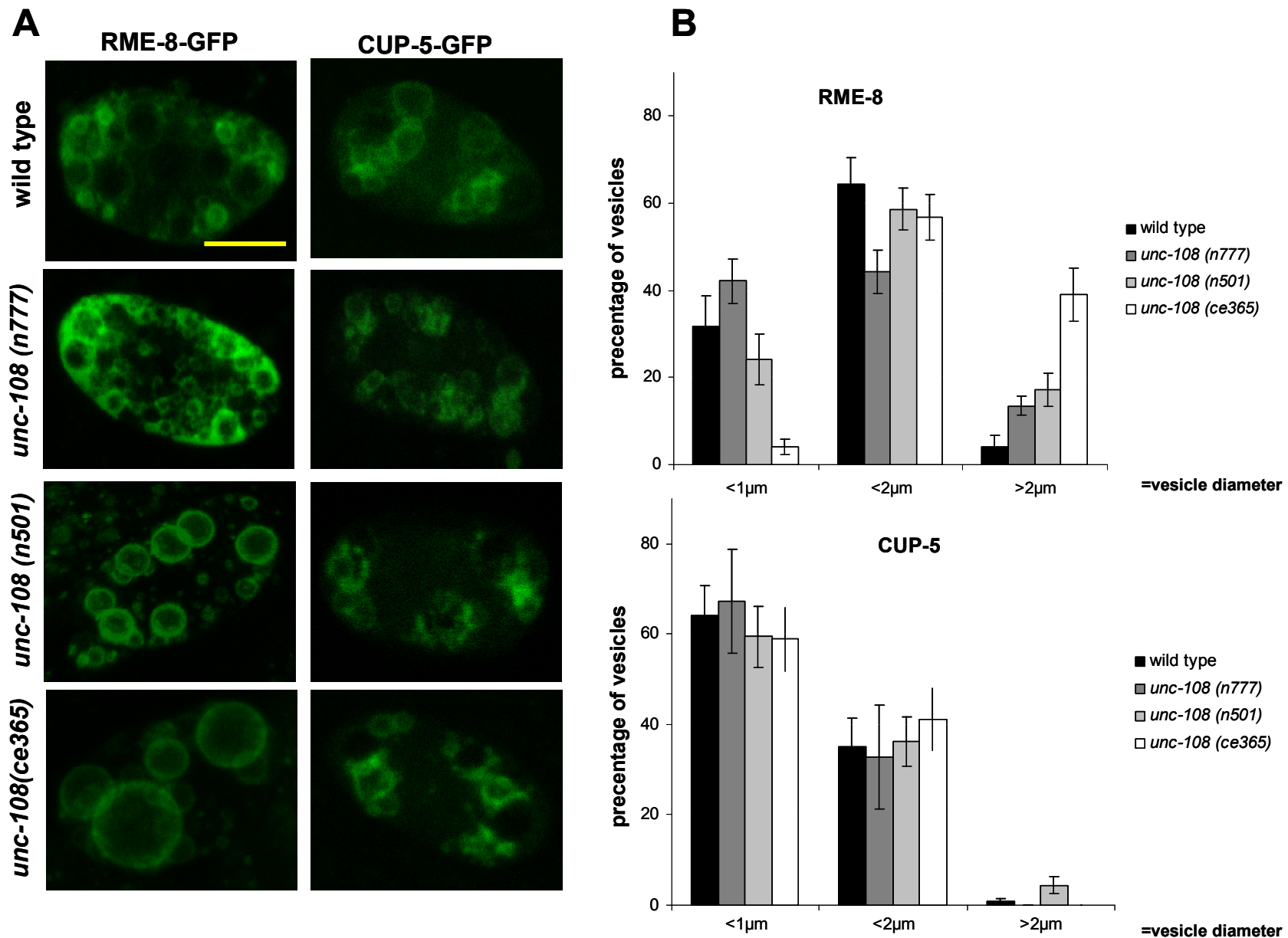

Figure 34. Gain of function unc-108 alleles, $n 501$ and $n 777$, have no defect in endocytotic pathway in coelomocytes.

(A) Localization of RME-8 (early and late endosomal marker) and CUP-5 (endosomal/lysosomal marker) in wild type and unc-108. Scale bar $2 \mu \mathrm{m}$.

(B) Size of RME-8 and CUP-5 positive vesicles in wild type and unc-108 mutants was sorted as a percentage of total number of vesicles into three categories based on their diameter, $<1 \mu \mathrm{m}, 1-2 \mu \mathrm{m}$ and $>2 \mu \mathrm{m}$. ce365 mutants have more of the large RME-8 positive vesicles (top), while in gain-of function alleles, $n 501$ and $n 777$ the endocytotic pathway seems to be normal (error bar=SEM, $\mathrm{N}=10$ animals per strain). 
coelomocytes
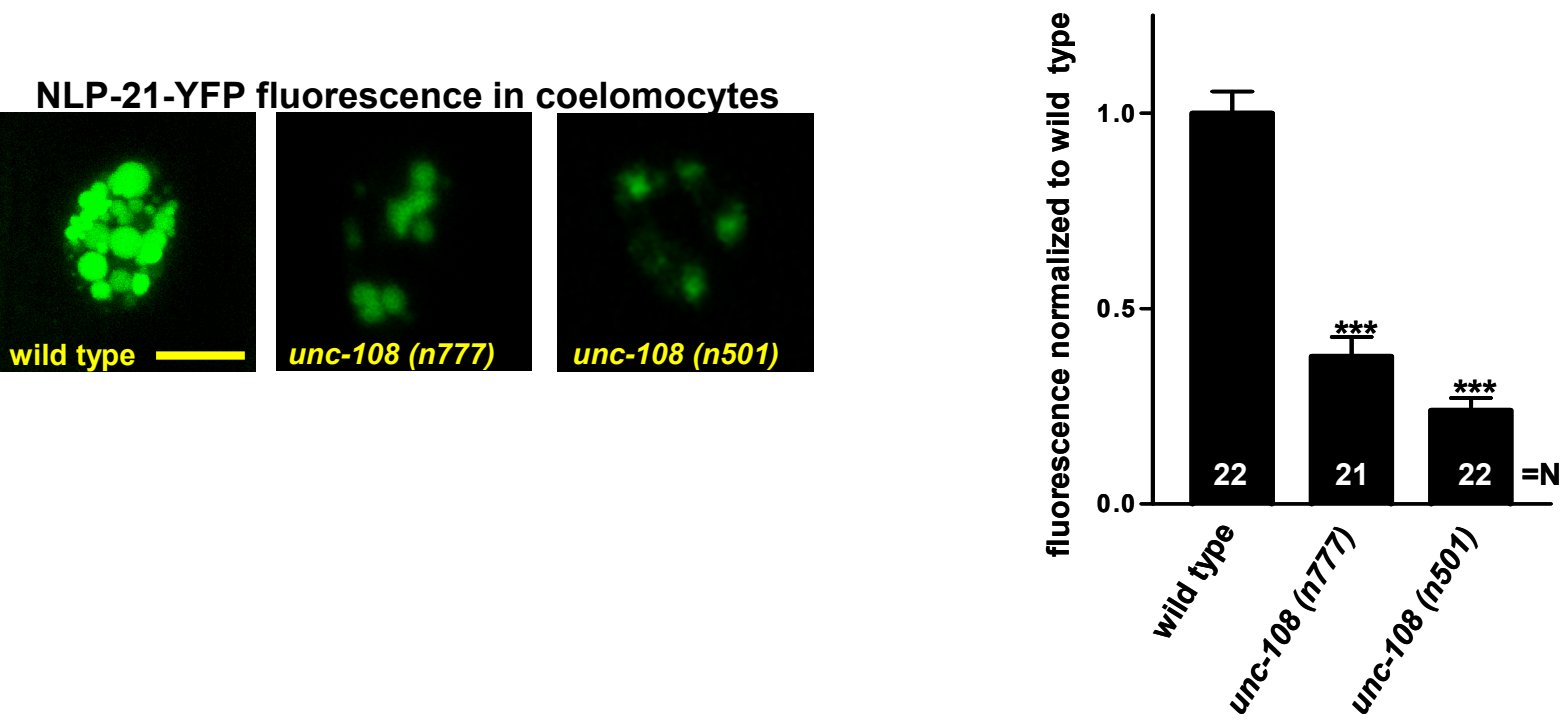

Figure 35. The neuropeptide secretion from cholinergic motorneuron synapses is strongly reduced in unc-108 mutants.

Neuropeptide NLP-21 was fused to YFP and stably expressed in cholinergic motorneurons in nuls183 strain. Once released from the synapse, NLP-21-YFP is taken up by scavenger cells coelomocytes. Left - Representative images of coelomocytes in wild type and unc-108 mutants. Right - Quantification of fluorescence intensity shows strong reduction of YFP content in unc-108 coelomocytes. Scale bar $5 \mu \mathrm{m}$. Number of animals analyzed is indicated in the graph bars. $\left({ }^{* * *} p<0.005\right.$, Student's T-test, error bar is SEM).

To eliminate the possibility that decrease in the NLP-21 derived YFP levels in axons is specific to the reporter construct used, we looked at the levels of another neuropeptide, INS-22, an insulin-like growth factor, also expressed in cholinergic motorneurons (Sieburth et al., 2007). In $n 501$ mutants the fluorescence of INS-22-YFP was significantly decreased by $60 \%$ (Fig. 36). Therefore, we conclude that decrease in neuropeptide levels at the synapses induced by mutations in RAB-2 are reporter construct independent and 
probably reflect that multiple soluble cargos are affected by unc-108 mutations.
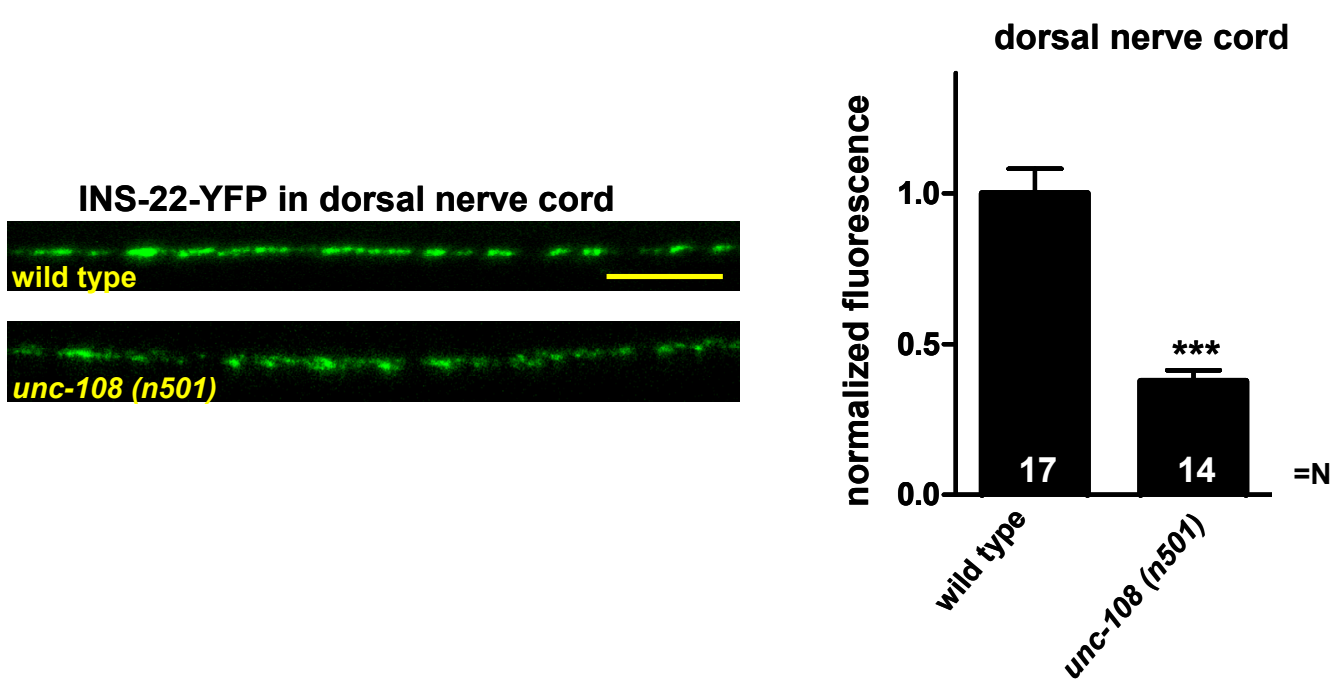

Figure 36. INS-22 insulin-like protein level is decreased at the synapses of unc-108 (n501) mutants.

INS-22 insulin-like growth factor was tagged to YFP and stably expressed in cholinergic motorneurons of nuls195 strain. The synapses located at the dorsal nerve cord were than imaged and the fluorescence was quantified. The YFP fluorescence shows $50 \%$ decrease in dominant unc-108 (n501) mutant, indicating decrease in neuropeptide levels at the synapses. (Number of animals analyzed is indicated in the graph bars, error bar is SEM, ${ }^{* * *} \mathrm{p}<0.005$, Student's T-test).

There are two possibilities that could explain the decrease in DCV derived YFP fluorescence in unc-108/rab-2 mutants: it can either come from changes in the DCV number or from lower amount of YFP packaged into DCVs. An HPF EM analysis of the DCV distribution at the synapse showed that the number of DCVs at the synapses is not changed in both gain- and loss-of function mutants. However, the size of the DCVs was more variable, especially for the loss of function mutant ce363 where the diameter of DCV is increased by $100 \%$ (Table 6). Therefore, it is most likely that the decrease in 
YFP fluorescence in DCVs reflects less YFP cargo packaged into DCVs in unc-108 mutants. This implements RAB-2 in DCV biogenesis.

\begin{tabular}{lcccc}
\hline Genotype & $\begin{array}{c}\text { Number of } \\
\text { profiles/animals } \\
\text { analyzed }\end{array}$ & $\begin{array}{c}\text { Average } \\
\text { synaptic } \\
\text { area }\left(\mu \mathrm{m}^{2}\right)\end{array}$ & DCV/profile & $\begin{array}{c}\text { Average } \\
\text { diameter of } \\
\text { DCVs }(\mathrm{nm})\end{array}$ \\
\hline wild type & $30 / 5$ & $0.25 \pm 0.02$ & $2.0 \pm 0.2$ & $44,8 \pm 1.0$ \\
unc-108 $(\mathrm{n777})$ & $34 / 4$ & $0.20 \pm 0.01$ & $2.0 \pm 0.2 \mathrm{~ns}$ & $54,1 \pm 3.4$ ** \\
unc-108 $(\mathrm{n} 501)$ & $31 / 4$ & $0.23 \pm 0.03$ & $2.3 \pm 0.3 \mathrm{~ns}$ & $49,4 \pm 2.3^{*}$ \\
unc-108 (ce363) & $28 / 3$ & $0.25 \pm 0.02$ & $1.2 \pm 0.3 *$ & $71,8 \pm 3.4$ *** \\
unc-108 (nu415) & $26 / 4$ & $0.21 \pm 0.01$ & $1.7 \pm 0.4 \mathrm{~ns}$ & $46,9 \pm 2.0 \mathrm{~ns}$ \\
rab-3 (js49) & $13 / 3$ & $0.22 \pm 0.02$ & $3.2 \pm 0.5 *$ & $41,7 \pm 1.1$ * \\
\hline
\end{tabular}

Table 6. DCV statistics for unc-108 mutants.

Statistics on DCV numbers and diameters as determined by HPF EM, the average synaptic area, number of synapses and independent animals analyzed are indicated. The DCV statistics of unc-108 mutants (highlighted in yellow) are compared to wild type $\left({ }^{*} p<0.05\right.$, ${ }^{* *} \mathrm{p}<0.01,{ }^{* * *} \mathrm{p}<0.005$, Student's T-test). (Courtesy of Jan Hegermann and Christian Olendrowitz)

\subsection{Neuropeptides are processed normally in unc-108/rab-2}

Besides the proneuropeptides, the enzymes responsible for their processing, furin type of proprotein convertases, carboxypeptidases and amidation enzymes are also packaged into the DCVs at the trans Golgi network (Husson et al., 2007). The processing of proneuropeptides occurs early in the DCV biogenesis, and the formed neuropeptides are concentrated and aggregated in the DCV. This process requires that the processing enzymes are sorted together with the proneuropeptides into the DCV, as well as that the DCV is 
properly acidified, in order for processing enzymes to be active (Kim et al., 2006; Morvan and Tooze, 2008). To check whether the processing is affected by mutations in RAB-2, we analyzed the neuropeptide content in protein extracts from unc-108 $n 501$ and $n 777$ alleles by HPLC and matrix-assisted laser desorption time-of-flight mass spectrometry (MALDI-TOF MS). It has been shown before that this method is sensitive enough to detect various products of proneuropeptide processing (Husson et al., 2006; Husson et al., 2007a). In unc-108 mutants, all the neuropeptides could be detected (Table 3). This applies to the neuropeptide like proteins, (NLP) type neuropeptides, as well as for the C-terminally modified FMRFamide like peptides (FLP) (Li et al., 1999). Thus, the processing of neuropeptides is not defective in unc-108 mutants. The MALDI-TOF MS method used to analyze and detect neuropeptide in protein extracts from $C$. elegans is not quantitative and allows therefore only qualitative conclusions.

\begin{tabular}{|c|c|c|c|c|}
\hline Gene & Peptide sequence & wild type & unc-108 (n777) & Unc-108 (n501) \\
\hline$n / p-1$ & VNLDPNSFRMSFa & $\mathrm{X} / \mathrm{X}$ & $\mathrm{X} / \mathrm{X}$ & $\mathrm{X} / \mathrm{X}$ \\
\hline$n / p-6$ & YKPRSFAMGFa & $\mathrm{X} /-$ & $\mathrm{X} /-$ & $-1-$ \\
\hline$n / p-6$ & AAMRSFNMGFa & $X /-$ & $\mathrm{X} /-$ & $-1-$ \\
\hline$n / p-7$ & MILPSLADLHRYTMYD & $X / X$ & $\mathrm{X} /-$ & $-/-$ \\
\hline$n / p-8$ & YPYLIFPASPSSGDSRRLV & $\mathrm{X} /-$ & $\mathrm{X} /-$ & $X / X$ \\
\hline$n / p-8$ & SFDRMGGTEFGLM & $X /-$ & $-/-$ & $-1-$ \\
\hline$n / p-9$ & TPIAEAQGAPEDVDDRRELE & $X / X$ & $\mathrm{X} /-$ & $-/-$ \\
\hline$n / p-11$ & SAPMASDYGNQFQMYNRLIDAa & $X / X$ & $\mathrm{X} /-$ & $-/-$ \\
\hline$n / p-11$ & HISPSYDVEIDAGNMRNLLDla & $X / X$ & $\mathrm{X} /-$ & $\mathrm{X} /-$ \\
\hline$n / p-11$ & SPAISPAYQFENAFGLSEALERAa & $X / X$ & $X / X$ & $X / X$ \\
\hline$n / p-12$ & DYRPLQFa & $X / X$ & $X / X$ & $X / X$ \\
\hline$n / p-12$ & DGYRPLQFa & $X / X$ & $X / X$ & $X / X$ \\
\hline$n / p-13$ & SPVDYDRPIMAFa & $X / X$ & $X / X$ & $-/-$ \\
\hline
\end{tabular}




\begin{tabular}{|c|c|c|c|c|}
\hline$n / p-13$ & SAPSDFSRDIMSFa & $X / X$ & $\mathrm{X} /-$ & $-/-$ \\
\hline$n / p-13$ & AEDYERQIMAFa & $\mathrm{X} /-$ & $\mathrm{X} /-$ & $-/-$ \\
\hline$n / p-14$ & ALNSLDGNGFGFD & $\mathrm{X} /-$ & $-/-$ & $-/-$ \\
\hline$n / p-14$ & ALNSLDGQGFGFE & $\mathrm{X} /-$ & $-1-$ & $-/-$ \\
\hline$n / p-16$ & NAEDHHEHQ & $X / X$ & $X / X$ & $X / X$ \\
\hline$n / p-18$ & SPYRTFAFA & $X / X$ & $X / X$ & $\mathrm{X} / \mathrm{X}$ \\
\hline$n / p-20$ & SGPQAHEGAGMRFAFA & $\mathrm{X} /-$ & $-/-$ & $-/-$ \\
\hline$n / p-21$ & GGARAFLTEM & $X /-$ & $\mathrm{X} /-$ & $-/-$ \\
\hline$n / p-21$ & GGARAFYDE & $X /-$ & $\mathrm{X} /-$ & $-/-$ \\
\hline$n / p-24$ & FTGPYGGYGa & $\mathrm{X} /-$ & $-/-$ & $-/-$ \\
\hline$n / p-35$ & AVVSGYDNIYQVLAPRF & $X / X$ & $X / X$ & $X / X$ \\
\hline$n / p-37$ & NNAEVVNHILKNFGALDRLGDVa & $X / X$ & $X / X$ & $\mathrm{X} /-$ \\
\hline$n / p-38$ & SPAQWQRANGLWa & $X / X$ & $X / X$ & $X / X$ \\
\hline$n / p-38$ & ASDDRVLGWNKAHGLWa & $X / X$ & $X / X$ & $-/-$ \\
\hline$n / p-39$ & EVPNFQADNVPEAGGRV & $X / X$ & $X / X$ & $-/-$ \\
\hline$n / p-40$ & APSAPAGLEEKLR & $X / X$ & $X / X$ & $\mathrm{X} /-$ \\
\hline$n / p-41$ & APGLFELPSRSVRLI & $X / X$ & $X / X$ & $X / X$ \\
\hline$f l p-1$ & SDPNFLRFa & $X / X$ & $X / X$ & $X / X$ \\
\hline$f \mid p-1$ & SADPNFLRFa & $X / X$ & $X / X$ & $X / X$ \\
\hline$f \mid p-1$ & AGSDPNFLRFa & $\mathrm{X} / \mathrm{X}$ & $X / X$ & $X / X$ \\
\hline$f l p-1$ & SQPNFLRFa & $X / X$ & $X / X$ & $x / X$ \\
\hline$f l p-2$ & SPREPIRFa & $X / X$ & $X / X$ & $\mathrm{X} /-$ \\
\hline$f l p-3$ & NPENDTPFGTMRFa & $X / X$ & $X / X$ & $-/-$ \\
\hline$f l p-3$ & NPLGTMRFa & $\mathrm{X} /-$ & $-/-$ & $-/-$ \\
\hline$f l p-3$ & SADDSAPFGTMRFa & $\mathrm{X} /-$ & $\mathrm{X} /-$ & $-/-$ \\
\hline$f l p-3$ & SAEPFGTMRFa & $\mathrm{X} /-$ & $\mathrm{X} /-$ & $-/-$ \\
\hline$f l p-3$ & ASEDALFGTMRFa & $\mathrm{X} /-$ & $\mathrm{X} /-$ & $-/-$ \\
\hline$f l p-4$ & ASPSFIRFa & $X / X$ & $X / X$ & $-/-$ \\
\hline$f l p-5$ & GAKFIRFa & $X / X$ & $X / X$ & $\mathrm{X} /-$ \\
\hline$f l p-5$ & AGAKFIRFa & $X / X$ & $X / X$ & $x / X$ \\
\hline$f l p-5$ & APKPKFIRFa & $X / X$ & $X / X$ & $X / X$ \\
\hline$f l p-6$ & KSAYMRFa & $X / X$ & $\mathrm{X} /-$ & $-/-$ \\
\hline$f l p-7$ & TPMQRSSMVRFa & $X / X$ & $X / X$ & $X / X$ \\
\hline flp-7 & SPMQRSSMVRFa & $X / X$ & $\mathrm{X} /-$ & $-/-$ \\
\hline$f l p-8$ & KNEFIRFa & $\mathrm{X} /-$ & $\mathrm{X} /-$ & $-/-$ \\
\hline$f l p-9$ & KPSFVRFa & $X / X$ & $X / X$ & $X / X$ \\
\hline$f \mid p-11$ & NGAPQPFVRFa & $X / X$ & $X / X$ & $X / X$ \\
\hline
\end{tabular}




\begin{tabular}{|c|c|c|c|c|}
\hline$f \mid p-11$ & AMRNALVRFa & $X / X$ & $\mathrm{X} /-$ & $\mathrm{X} / \mathrm{X}$ \\
\hline$f \mid p-11$ & ASGGMRNALVRFa & $X / X$ & $X / X$ & $X / X$ \\
\hline$f l p-12$ & RNKFEFIRFa & $X /-$ & $-/-$ & $-/-$ \\
\hline$f / p-13$ & AADGAPLIRFa & $X / X$ & $X / X$ & $\mathrm{X} / \mathrm{X}$ \\
\hline$f l p-13$ & ASSAPLIRFa & $x / X$ & $X / X$ & $X / X$ \\
\hline$f l p-13$ & APEASPFIRFa & $X / X$ & $X / X$ & $\mathrm{X} / \mathrm{X}$ \\
\hline$f / p-13$ & ASPSAPLIRFa & $X / X$ & $X / X$ & $X / X$ \\
\hline$f l p-13$ & SPSAVPLIRFa & $X / X$ & $X / X$ & $\mathrm{X} / \mathrm{X}$ \\
\hline$f / p-14$ & KHEYLRFa & $X / X$ & $X / X$ & $\mathrm{X} / \mathrm{X}$ \\
\hline$f l p-15$ & GGPQGPLRFa & $X / X$ & $X / X$ & $X / X$ \\
\hline$f l p-15$ & RGPSGPLRFa & $X / X$ & $X / X$ & $\mathrm{X} / \mathrm{X}$ \\
\hline$f / p-16$ & GQTFVRFa & $X / X$ & $X / X$ & $\mathrm{X} / \mathrm{X}$ \\
\hline$f / p-16$ & AQTFVRFa & $X / X$ & $X / X$ & $X / X$ \\
\hline$f \mid p-17$ & KSAFVRFa & $X / X$ & $\mathrm{X} /-$ & $\mathrm{X} / \mathrm{X}$ \\
\hline$f / p-18$ & DFDGAMPGVLRFa & $X / X$ & $X / X$ & $X / X$ \\
\hline$f / p-18$ & EIPGVLRFa & $X / X$ & $X / X$ & $X / X$ \\
\hline$f / p-18$ & EMPGVLRFa & $X / X$ & $X / X$ & $-/-$ \\
\hline$f / p-18$ & SEVPGVLRFa & $X / X$ & $X / X$ & $X / X$ \\
\hline$f / p-18$ & DVPGVLRFa & $X / X$ & $\mathrm{X} /-$ & $X / X$ \\
\hline$f / p-18$ & SVPGVLRFa & $X / X$ & $X / X$ & $\mathrm{X} / \mathrm{X}$ \\
\hline$f l p-19$ & WANQVRFa & $X / X$ & $X / X$ & $X / X$ \\
\hline flp-19 & ASWASSVRFa & $X / X$ & $X / X$ & $-/-$ \\
\hline$f l p-22$ & SPSAKWMRFa & $X / X$ & $\mathrm{X} /-$ & $\mathrm{X} /-$ \\
\hline$f / p-24$ & VPSAGDMMVRFa & $\mathrm{X} / \mathrm{X}$ & $\mathrm{X} / \mathrm{X}$ & $-/-$ \\
\hline$f / p-25$ & AAPIKKASYDYIRFa & $\mathrm{X} / \mathrm{X}$ & $X / X$ & $\mathrm{X} /-$ \\
\hline$f l p-26$ & EFNADDLTLRFa & $X / X$ & $X / X$ & $-/-$ \\
\hline$f \mid p-26$ & GGAGEPLAFSPDMLSLRFa & $X / X$ & $\mathrm{X} / \mathrm{X}$ & $X / X$ \\
\hline$f / p-28$ & APNRVLMRFa & $X / X$ & $X / X$ & $-/-$ \\
\hline$f \mid p-33$ & APLEGFEDMSGFLRTIDGIQKPRFa & $X / X$ & $X / X$ & $-/-$ \\
\hline
\end{tabular}

Table 7. HPLC-MALDI TOF MS analysis of wild type and unc-108 mutants

Protein extracts from unc-108 $n 501$ and $n 777$ alleles by HPLC and matrix-assisted laser desorption time-of-flight mass spectrometry (MALDI-TOF MS). Two trials were performed for each strain. $X$ stands for each time peptide was detected for each trial. (Courtesy of Steven Husson). 


\subsection{RAB-2 affects DCV signaling parallel to neuropeptides}

To test whether mutations in RAB-2 affect levels of functional neuropeptides, we analyzed double mutants of unc-108 with egl-3, a proprotein convertase 2 (PC2) homolog required for proteolytical cleavage of neuropeptides from their precursor (Kass et al., 2001). In the absence of EGL-3/PC2 activity, no active neuropeptides are detectable (Husson et al., 2006). egl-3 mutants also show impaired movement and aldicarb resistance (Fig. 37 and Sieburth et al., 2005). If EGL-3 and UNC-108/RAB-2 were involved in the same pathway, required for neuropeptide signaling then an egl-3;unc-108 double mutant should show the same phenotype as egl-3 single mutants.

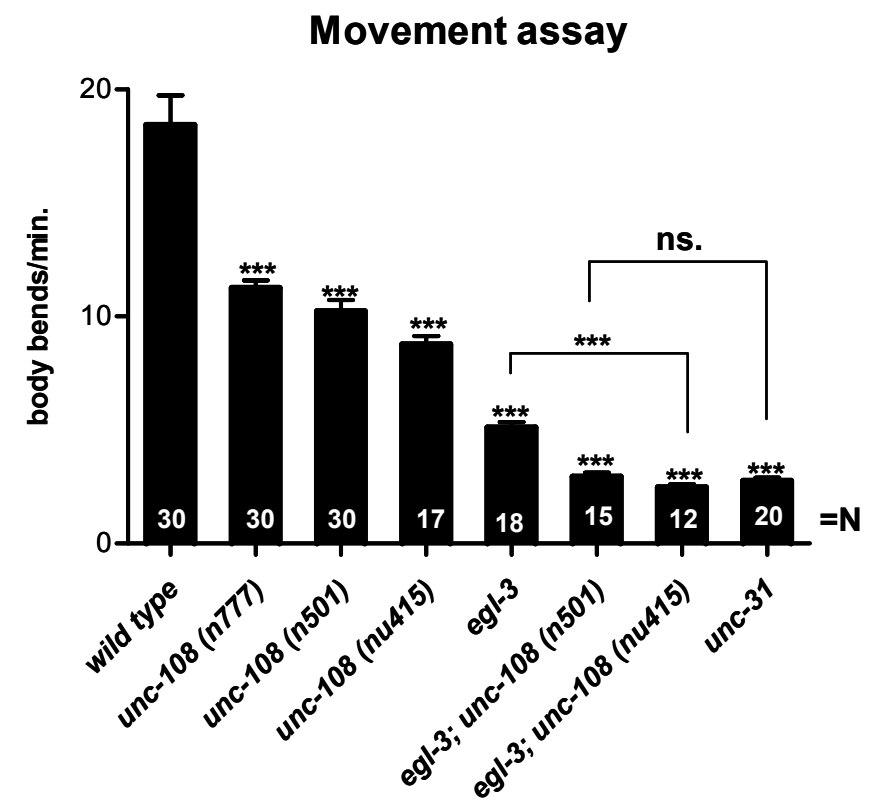

Figure 37. Deletion of proprotein convertase egl-3 in unc-108 mutants leads to phenotype similar to the one observed in unc-31 mutants.

Deletion of PC2 homologue EGL-3 causes a movement defect, which becomes more severe and similar to unc-31 in unc-108 mutant background, $\left({ }^{* * *} \mathrm{p}<0.005\right.$, Student's t-test, all strains were compared to wild type, double mutants are compared to egl-3 and unc-31 single mutants. Number of animals analyzed is indicated in the graph bars, error bar= SEM). 
However, egl-3;unc-108 double mutants show a strong enhancement of the locomotory defects reducing the locomotion of the double mutant to the level of unc-31/CAPS mutants which are defective in DCV release (Fig. 37). Both dominant and recessive unc-108 alleles show the same strong synthetic interaction with egl-3. This suggests that unc-108 and egl-3 are acting in parallel pathways for DCV signaling.

\subsection{RAB-2 localizes to the Golgi, but not at the synapses}

To understand how RAB-2 is involved in DCV biogenesis or maturation we studied its subcellular localization.
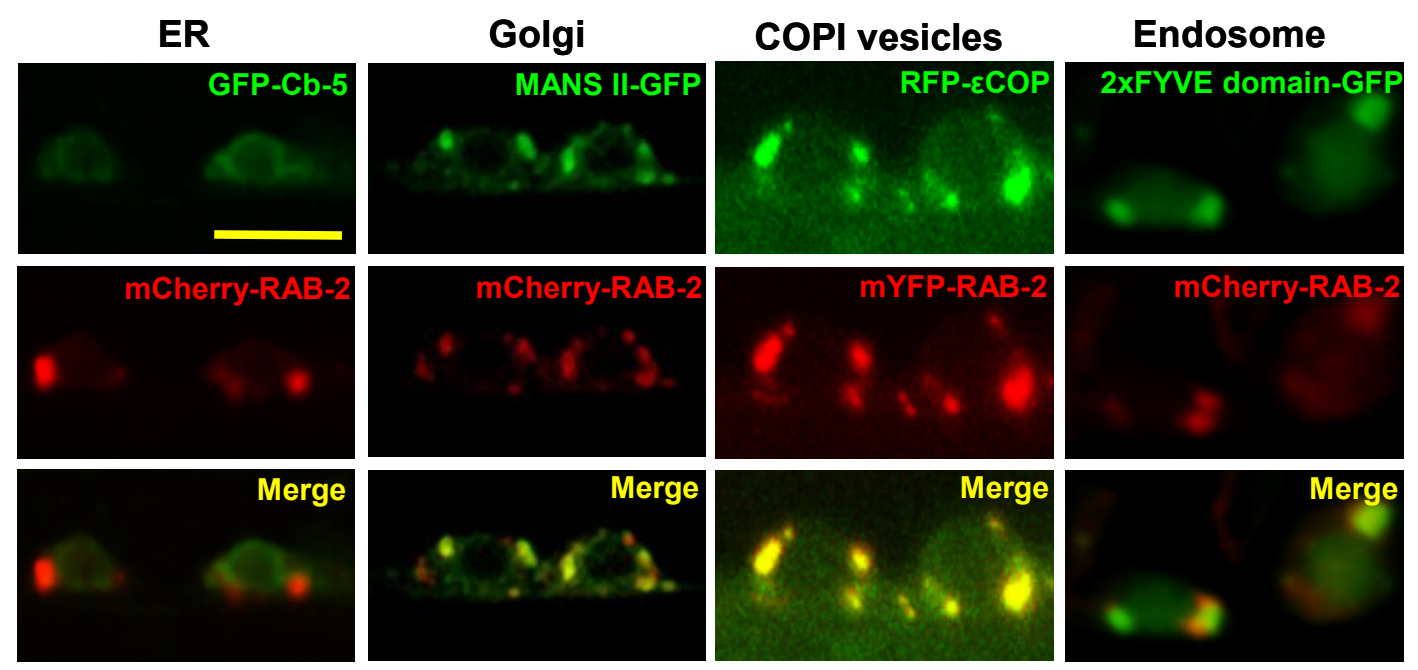

Figure 38. RAB-2 localizes to the Golgi apparatus

Fluorescently tagged RAB-2 protein was expressed in the nervous system along with different intracellular markers. Shown are confocal images of neuronal cell bodies in the ventral nerve cord. RAB-2 extensively colocalizes with Golgi markers Mannosidase II (MANS II) and COPI

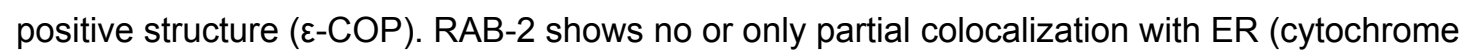
b5, Cb-5) or early endosomes (FYVE-domain of EEA-1). (Scale bar $5 \mu \mathrm{m}$ ). 
When expressed as a fusion protein tagged N-terminally with either mYFPcitrine or mCherry, RAB-2 localizes to discrete cytoplasmic puncta in cell bodies of motorneurons (Fig. 38). Localization studies of RAB-2 showed its strong colocalization with Golgi marker Mannosidase II, while there was no colocalization with ER marker Cytochrome-b5 nor endosomal marker, FYVE domain of RAB-5 effector EEA-1.

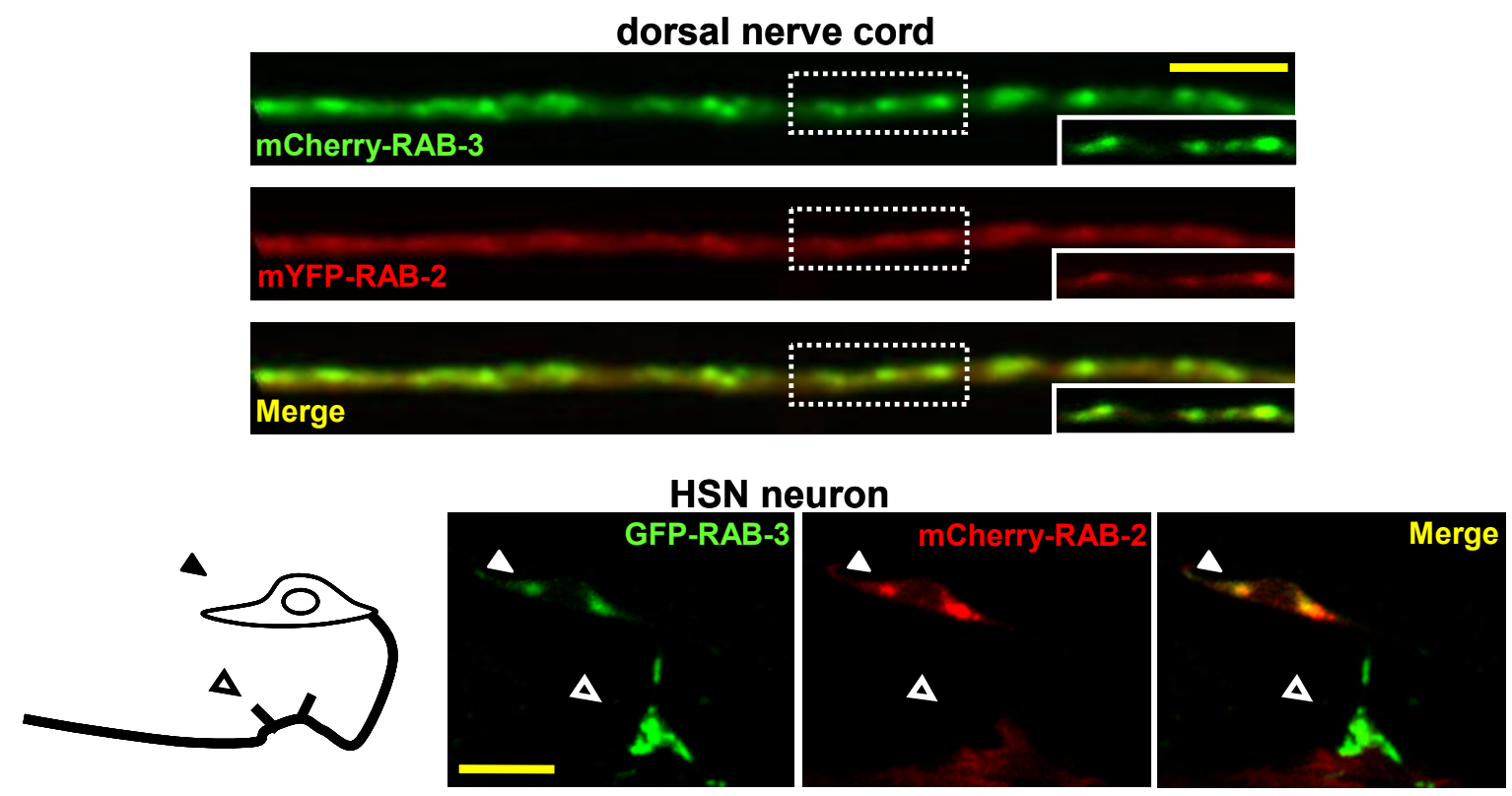

Figure 39. RAB-2 is not present at the synapses

mYFP-RAB-2 (shown in red) does not accumulate at the synapses in the dorsal nerve cord like mCherry-RAB-3 (shown in green). The boxes represent the magnified synaptic punctae demonstrating no accumulation of RAB-2 at the synapses (Scale bar $10 \mu \mathrm{m}$ ). Schematic drawing on the left represents the HSN neuron. The closed arrow heads represent the cell body of HSN neuron and the open arrowheads, the synapses formed by HSN neuron onto the vulval muscle and VC4 and VC5 neurons. HSN neuron axon enters the ventral nerve cord and stretches towards the head of the worm. In HSN neurons mCherry-RAB-2 is present only in cytoplasmic puncta in neuronal cell body, but not at the synapse with vulva muscle. HSN synapses are labeled by GFP-RAB-3. Scale bar is $5 \mu \mathrm{m}$. 
More importantly, RAB-2 does not localize to the synapses, as seen on light microscopy images. While for RAB-3 protein, which is a SV marker, strong accumulations were observed at the synapses of dorsal nerve cord, no accumulation was observed for RAB-2. Furthermore, looking at the single HSN neuron, strong accumulation of RAB-3 was observed at the synapse of the neuron with the vulva muscle, while RAB-2 is present only at the cell body of the neuron (Fig. 39).

To confirm the Golgi localization of the RAB-2, we performed immunogold labeling of endogenous RAB-2 on thin plastic sections immuno EM. For this purpose we generated monoclonal antibodies against $C$. elegans RAB-2. RAB-2 broadly localizes to Golgi and vesicular structures around the Golgi with preference for cis side (Fig. 40). The staining was specific, because for rab-2 deletion mutant no gold labels were observed (Fig. 41). This suggests RAB-2 is active at the Golgi and not at the synapse, which is in agreement with possible function of RAB-2 in DCV biogenesis or maturation.

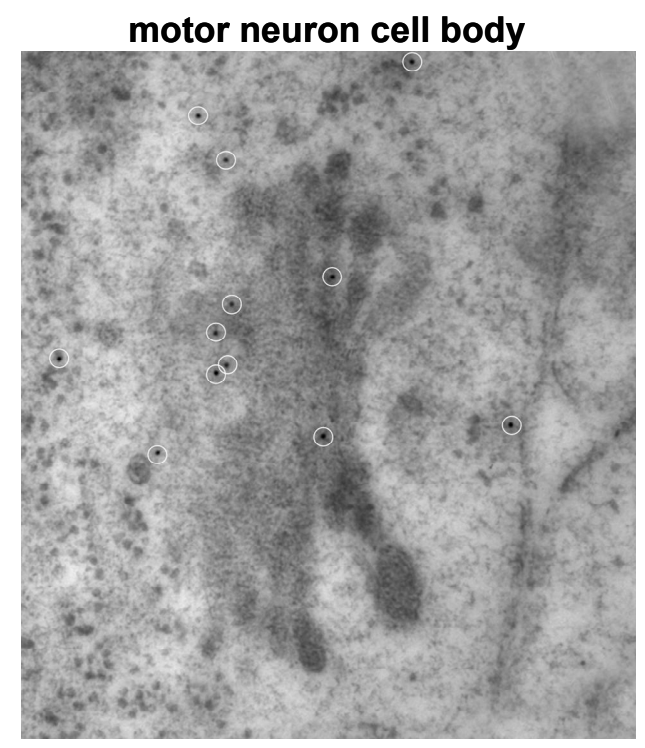

Figure 40. RAB-2 is enriched at the cis side of the Golgi.

Immuno-gold labeling of endogenous RAB-2 in motorneuron cell bodies. $85 \mathrm{~nm}$ HPF EM thin plastic sections were probed with monoclonal RAB-2 antibodies. Gold labeling (white circles) is seen at the Golgi complex and vesicular structures around the Golgi. (Courtesy of Jan Hegermann). 


\section{motorneuron cell bodies}

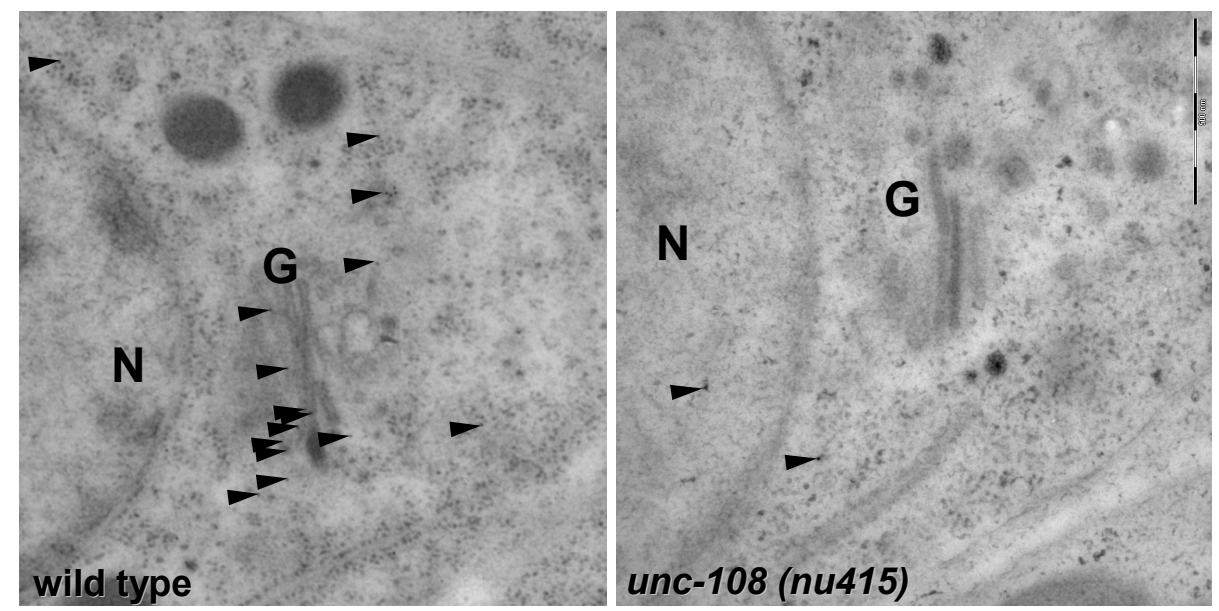

Figure 41. Immunogoldlabeling of endogenous RAB-2 in wild type and null unc-108 (nu415) strain.

Immunogoldlabelling of endogenous RAB-2 in wild type and null unc-108 (nu415) strain, showing the specificity of the RAB-2 antibody. While in wild type most of the labels are at the Golgi or at the vesicles around the Golgi, in nu415 allele which has a nonsense mutation in rab-2 gene, only the background stains are observed. N, nucleus; G, Golgi stack. (Courtesy of Jan Hegermann).

Because the interaction of RAB-2 with Golgi matrix protein and its localization at the Golgi, one possibility would be that mutations in RAB-2 influence the morphology and therefore the function of the Golgi. Ultimately, this would influence the DCV formation at the trans Golgi network. However, the Golgi complexes appeared to be normal in unc-108 mutants (Fig. 43). 


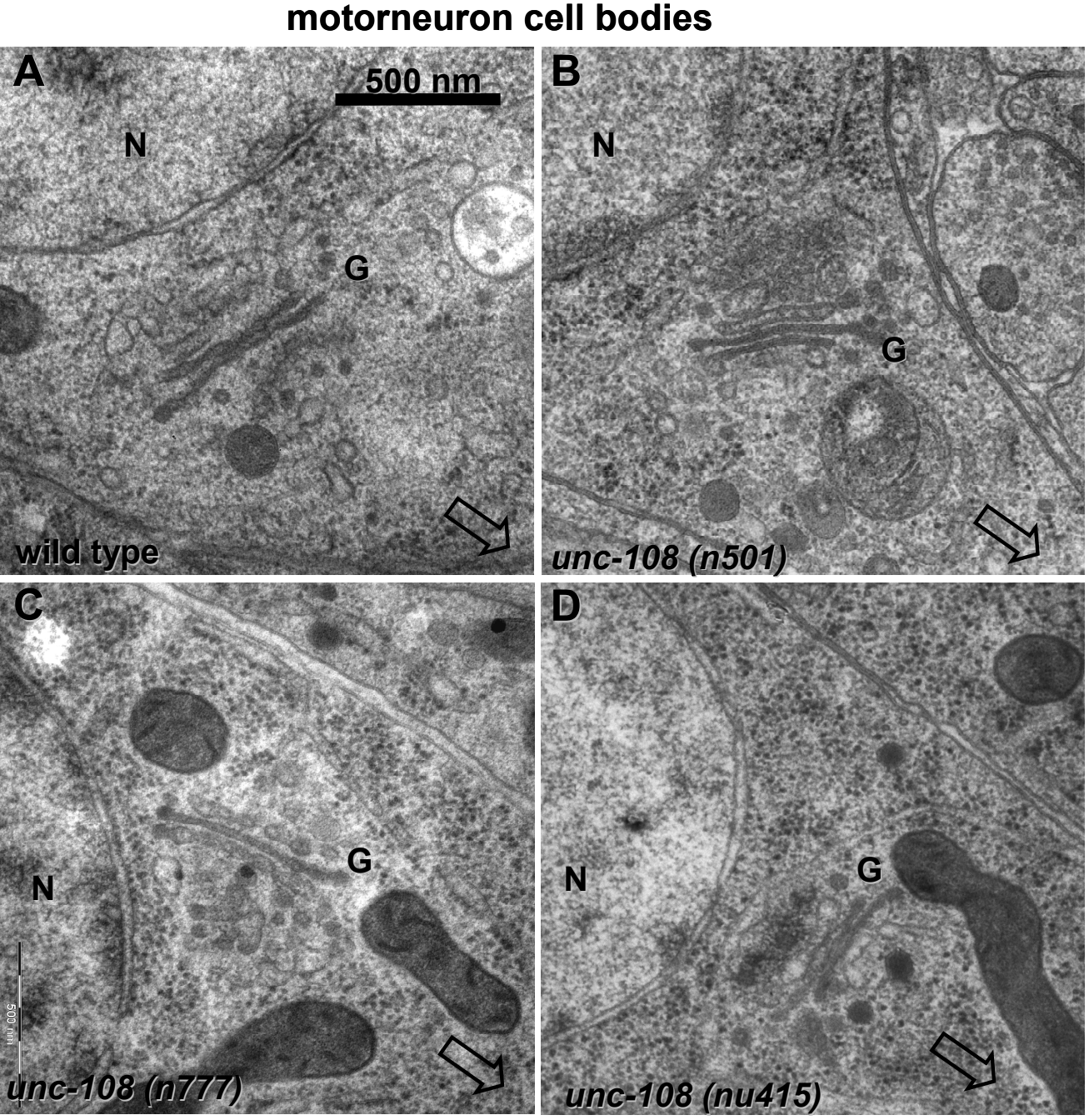

Figure 43. Golgi morphology in neuronal cell bodies is not altered in unc-108 mutants.

Motorneuron cell bodies are shown for wild type (A) and unc-108 n501 (B), $n 777$ (C) and nu415 (D) alleles. The morphology of the Golgi complexes in dominant or recessive unc-108 mutants appears normal when compared to wild type using HPF EM. (Courtesy of Jan Hegermann). 


\subsection{RAB-2 mutations affect DCV maturation}

Since the DCV number is not changed in unc-108 mutants based on EM, we wanted to ask how changes in UNC-108/RAB-2 affect the levels of NLP-21 derived YFP in DCVs.

A
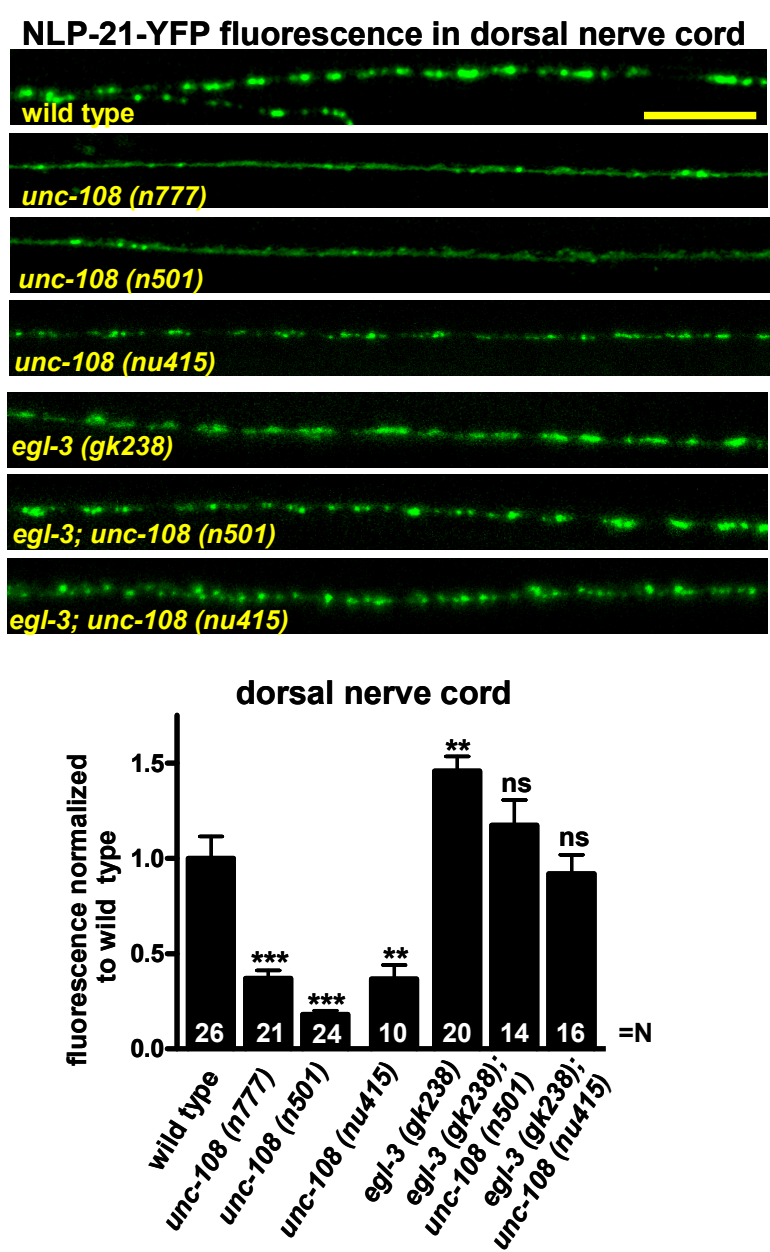

B
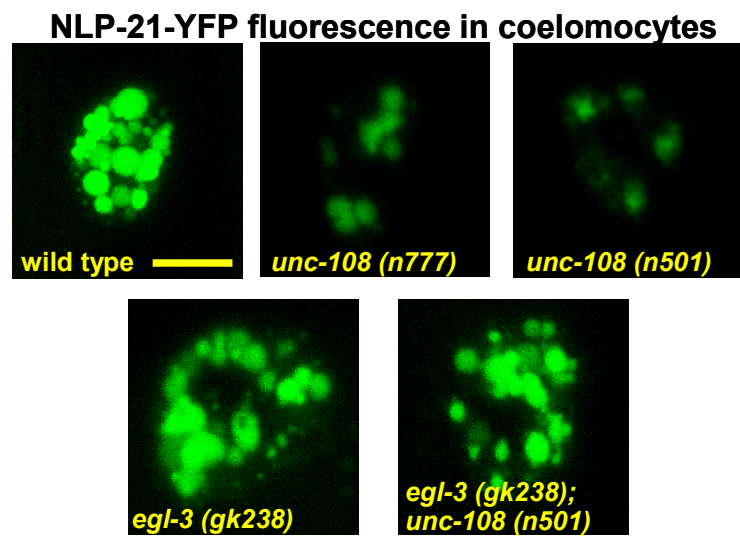

Figure 44. unc-108 mutants have strongly reduced soluble cargo in DCVs at the synapses.

(A) Animals carrying integrated array nuls183 are expressing NLP-21-YFP in motorneurons projecting axons into the dorsal nerve cord. Shown are representative images of the synaptic accumulation of DCVs with YFP tagged NLP-21 neuropeptide in dorsal nerve cord in the respective genetic backgrounds. Average fluorescence was normalized to wild type (graph bellow). The absence of EGL-3 leads to strong accumulation of neuropeptides at the synapse 
and restores wild type levels in both dominant and recessive unc-108 alleles. (Scale bar $10 \mu \mathrm{m},{ }^{* * *} p<0.005,{ }^{* *} \mathrm{p}<0.01$, Student's T-test, error bar= SEM, $\mathrm{N}$ is indicated in the graph bars)

(B) Once released at the synapse, NLP-21-YFP is taken up by coelomocytes. Shown are representative images of coelomocytes in different genetic backgrounds. Average fluorescence was normalized to wild type (graph bellow). (Scale bar $5 \mu \mathrm{m}, \mathrm{N}$ is indicated in the graph bars, error bar is SEM, ${ }^{* * *} p<0.005$, Student's T-test).

NLP-21 derived YFP levels at the synapses of egl-3 mutants are increased when compared to wild type. This is probably due to the fact that most of the YFP remains bound to the proneuropeptide in the dense core of the vesicle (Fig. 44A). The unc-108 neuropeptide levels at the synapses in egl-3 background are similar to the wild type levels. This is followed by increased fluorescence in coelomocytes (Fig. 44B). This suggests that the loss of the neuropeptides occurs after the neuropeptide processing. In a parallel study, Edwards et al. demonstrated that the neuropeptide levels are not changed in unc-108 mutants. However soluble cargo, like NLP-21 derived YFP is lost from DCVs. The fact that unprocessed NLP-21-YFP is retained in DCVs of unc-108 mutants supports this finding. Neuropeptides have been shown to aggregate in DCVs and are thus likely protected from loss in unc-108 mutants (Kim et al, 2006; Tooze et al., 2001).

We therefore reasoned that unc-108/rab-2 mutations preferentially affect soluble cargo or cargo that is free to diffuse out of the DCVs during the maturation process. During the sorting and packaging process at the trans Golgi network, constitutive secretory cargo, lysosomal enzymes, and other membrane proteins are co-packaged into DCV precursors, forming iDCV. To 
become fully functional, this inappropriate cargo has to be removed from iDCVs by clathrin-mediated membrane trafficking and delivered to the endosomal system. This remodeling process allows iDCV maturation into a mDCV.

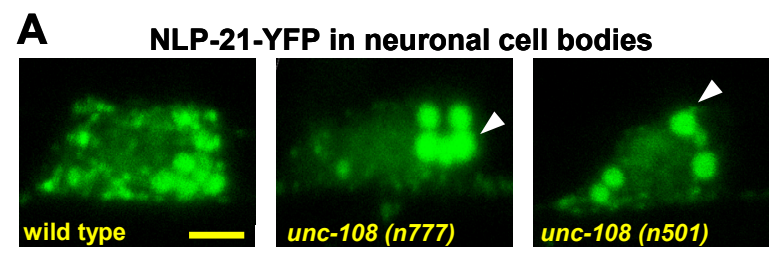

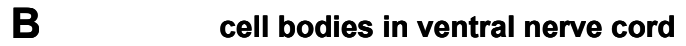

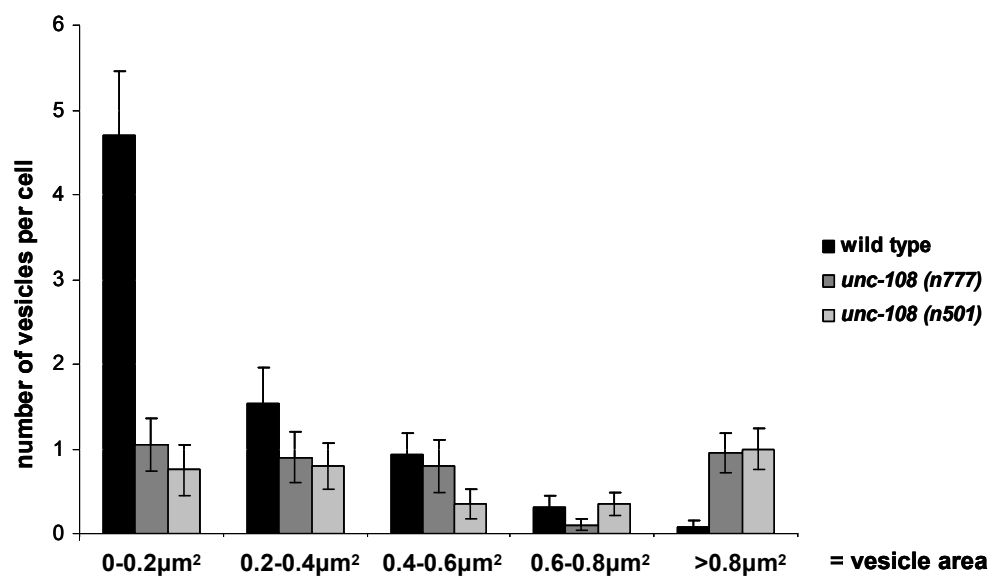

C cell bodies in ventral nerve cord

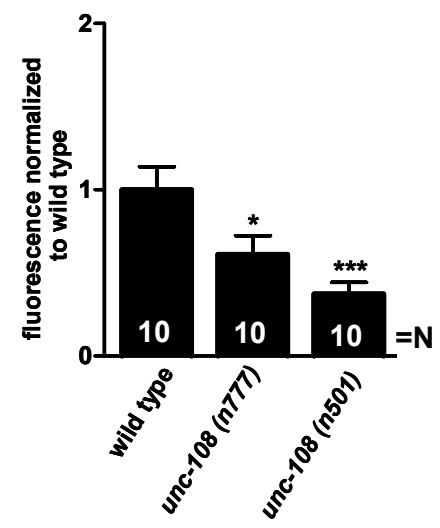

Figure 45. unc-108 mutants have increase in the size of NLP-21-YFP positive vesicles in neuronal cell bodies.

(A) and (B) NLP-21-YFP was stably expressed in cholinergic motorneurons in the strain nuls183 and crossed into unc-108 mutants. In wild type animals, the size of most of the NLP21-YFP positive vesicular structures present in the neuronal cell bodies is $0.2 \mu \mathrm{m}^{2}$, while in unc-108 mutants these small vesicles disappear. Most of the vesicles observed in unc-108 soma is larger in size compared to wild type. Scale bar is $1 \mu \mathrm{m}$. (error bar is SEM, 10 animals were analyzed for each strain). 
(C) The amount of neuropeptide present in the neuronal cell bodies of unc-108 mutants is decreased compared to wild type. $\left({ }^{*} p<0.05,{ }^{* * *} p<0.005\right.$, Student's T-test, error bar is SEM, N is indicated in the graph bars)

To test whether the mutations in UNC-108/RAB-2 affect the DCV maturation, we looked at the NLP-21-YFP staining in the neuronal cell bodies. If the hypothesis is correct, one would expect aberrant accumulations of NLP-21YFP in the cell bodies. NLP-21-YFP shows punctuate staining in the soma, with numerous small vesicular structures filling the cytoplasm (Fig. 45A and B). In unc-108 mutants however, the size of NLP-21-YFP positive vesicular structures is significantly enlarged and reaches the size of over $0.8 \mu \mathrm{m}^{2}$, never observed in the wild type. Moreover, the small vesicular structures that are quite abundant in wild type soma almost completely disappear from unc-108 cytoplasm. The YFP fluorescence in unc-108 somas is significantly reduced (Fig. 45C). This is in agreement with our hypothesis that following the expected cleavage of the NLP-21-YFP, the YFP fusion protein is less efficiently retained in iDCVs of unc-108 mutants. Thus, NLP-21 derived YFP might be largely removed during the maturation process by sorting to the endosomal system.

To test this possibility, we colocalized the NLP-21-YFP fusion with various subcellular markers in order to identify the nature of the enlarged vesicular structures: syntaxin-6, a protein involved in iDCV homotypic fusion (Morvan and Tooze, 2008; Wendler et al., 2001), RAB-5, an early endosomal marker, RAB-7, a late endosomal marker and LMP-1, a lysosomal marker (Kostich et al., 2000). In wild type neuronal cell bodies of the ventral cord, most of the 
YFP fluorescence overlapped with larger structures positive for RAB-2 and smaller vesicular structures that are positive for the early endosomal marker RAB-5 (Fig. 46A). Almost no overlap is seen with the late endosomal marker RAB-7 as well as the lysosomal marker LMP-1. This suggests that in wild type cells most of the NLP-21-YFP fusion proteins accumulate at the Golgi and early endosomes.

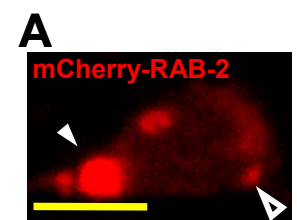

neuronal cell bodies in wild type ventral nerve cord
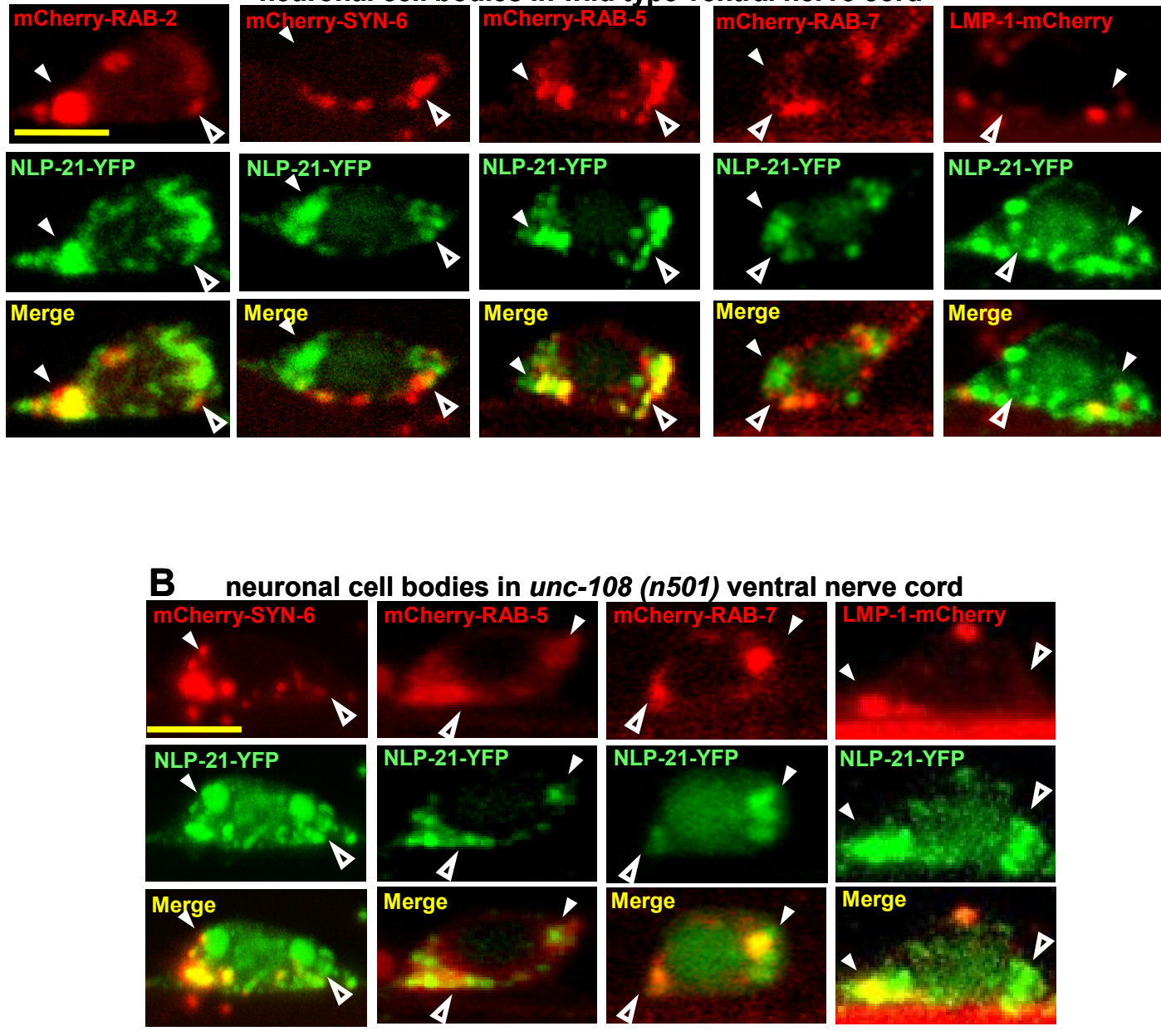

Figure 46. In unc-108 NLP-21-YFP enters late endosomal compartment.

To identify the NLP-21-YFP positive vesicular structures in wild type (A) and unc-108 (n501) strains (B) carrying the NLP-21-YFP integrated array, different intracellular markers were expressed pan-neuronally: RAB-2, syntaxin 6 (iDCVs), RAB-5 (early endosome), RAB-7 (late endosome) and LMP-1 (lysosome). The solid and open arrow heads represent larger and 
smaller vesicular structures respectively. NLP-21-YFP positive vesicles in unc-108 (n501) strain mostly colocalize with early endosomal marker RAB-5. Scale bar $2 \mu \mathrm{m}$.

This picture changes dramatically in the dominant unc-108 mutants where NLP-21-YFP largely overlaps with markers for late endosomes and lysosomes, RAB-7 and LMP-1 (Fig. 46B). This supports the idea that in dominant unc-108 mutants the NLP-21 derived YFP is partially lost after proneuropeptide processing from maturing DCVs entering, via trafficking into the late endosomal-Iysosomal degradation route.

To test whether indeed soluble cargo is lost in the endosomal/lysosomal system in unc-108 mutants, we aimed to block endosomal delivery by interfering with early endosomal function. It has been shown that the overexpression of dominant active form of RAB-5 blocks the endosomal function by preventing the maturation of early into the late endosome(Simonsen et al., 1998). If our hypothesis was correct, the blockage of endosomal/lysosomal route would restore the wild type levels of neuropeptide at the unc-108 synapses. As seen in Fig. 47 the YFP fluorescence levels at the synapse were specifically rescued in unc-108 mutants. In contrast expression of RAB-5 (Q78L) DA in wild type motorneurons had no effect on axonal levels of the NLP-21-YFP fluorescence.

In agreement with this, the NLP-21 derived YFP fluorescence was also rescued to wild type levels in neuronal cell bodies of unc-108 (n501) by expression of RAB-5 (Q78L) DA (Fig. 48). These results confirm that RAB-2 
activity is required during DCV maturation to retain cargo in maturing DCVs by preventing its entry to the late endosomal system.
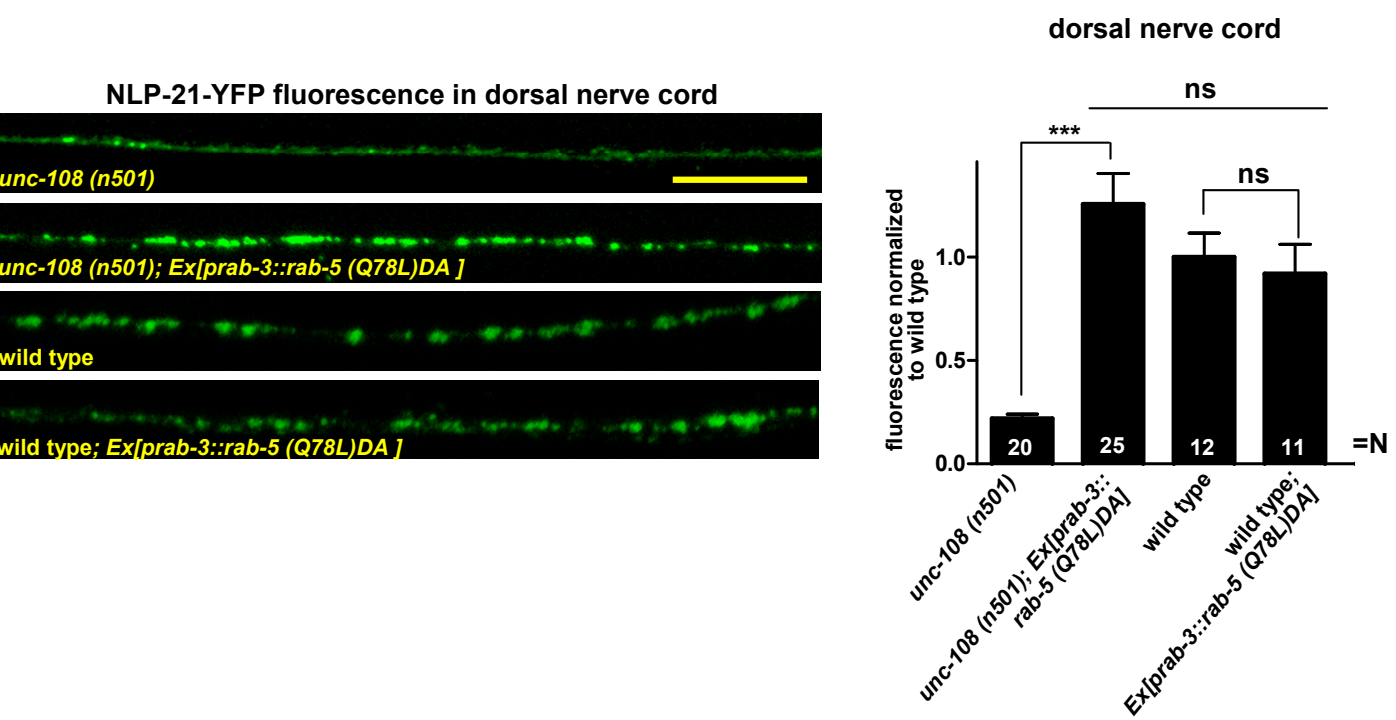

Figure 47. Overexpression of RAB-5 GTP bound form increases the amount of neuropeptides at the unc-108 synapses.

RAB-5 GTP bound form, RAB-5(Q78L)DA, was expressed pan-neuronally in NLP-21-YFP expressing strain nuls183 in wild type and unc-108 (n501) background. Presence of RAB5(Q78L)DA correlates to much higher level of NLP-21-YFP at the synapses in unc-108 (n501) strain, while in the wild type background the amount of the neuropeptide at the synapse does not change. Average fluorescence was normalized to wild type. (Scale bar $10 \mu \mathrm{m},{ }^{* * *} \mathrm{p}<0.005$, Student's T-test, error bar = SEM, N is indicated in the graph bars).

The overexpression of dominant active GTP bound form of Rab-5 leads to formation of big vesicular structures in the neuronal cell bodies (Fig. 49A), which overlaps with NLP-21-YFP positive vesicles. At the EM level, these structures look like large multivesicular bodies (Fig. 49B), which supports the hypothesis that overexpression of dominant active GTP bound RAB-5 drives the fusion of early endosomes and blocks the maturation of the endosomal system. 
NLP-21-YFP fluorescence in the cell bodies in the ventral nerve cord
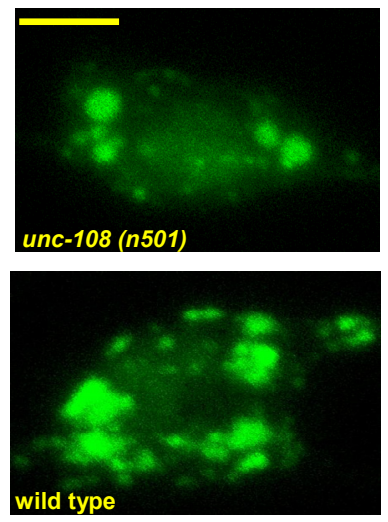
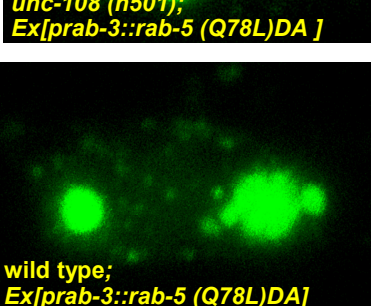

cell bodies

in the ventral nerve cord

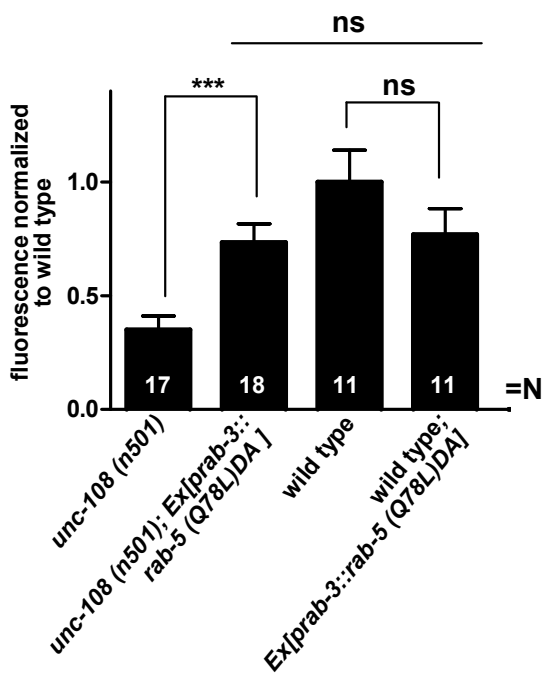

Figure 48. Overexpression of dominant active GTP bound RAB-5 (Q78L) DA increases the amount of neuropeptides in the unc-108 neuronal cell bodies.

Overexpression of dominant active GTP bound RAB-5 (Q78L) DA in nuls183 strain which stably expresses neuropeptide NLP-21-YFP in cholinergic motorneurons, leads to accumulation of neuropeptide in the neuronal cell bodies in the ventral nerve cord. (Scale bar $2 \mu \mathrm{m},{ }^{* * *} \mathrm{p}<0.005$, Student's T-test, error bar=SEM, $N$ is indicated in the graph bars).

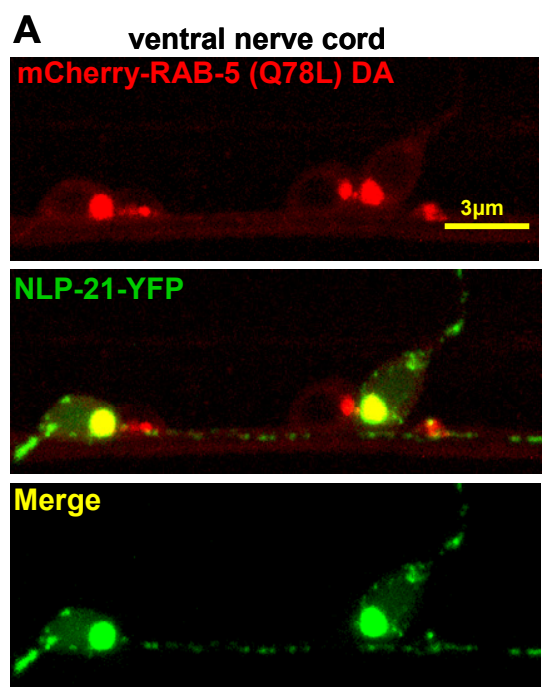

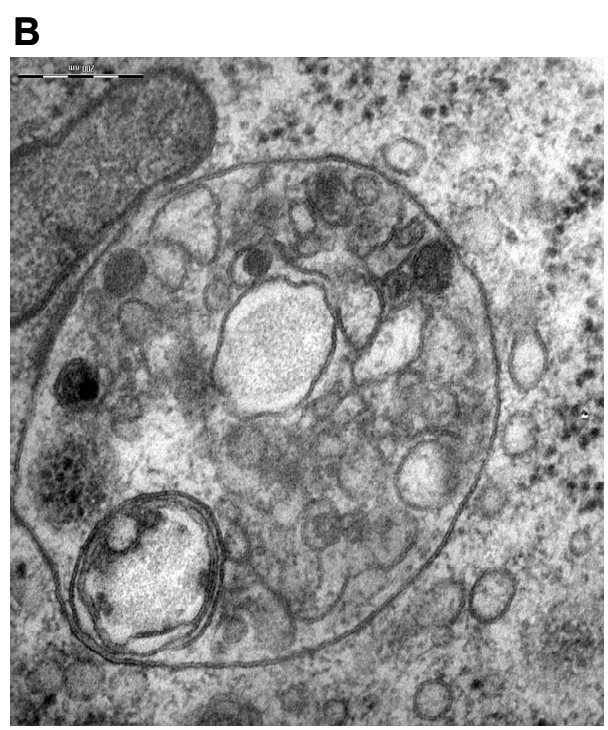

$200 \mathrm{~nm}$

Figure 49. Overexpression of dominant active GTP bound form of RAB-5 leads to formation of multivesicular bodies in neuronal soma.

(A) mCherry tagged dominant active GTP bound RAB-5 was expressed under rab-3 promoter in nuls183 strain, which stably expresses neuropeptide NLP-21 tagged to YFP in cholinergic 
motorneurons. The overexpression of RAB- 5 leads to formation of big vesicular structures in neuronal soma. mCherry fluorescence perfectly overlaps with YFP fluorescence of NLP-21. Most of the small vesicles present in the wild type soma disappear upon the expression of RAB-5 dominant active form. Scale bar is $3 \mu \mathrm{m}$.

(B) Electron micrograph of the vesicular structures formed upon the overexpression of dominant active GTP bound RAB-5 appear to be multivesicular bodies, which are formed probably due to the blockage of maturation of early endosome to late endosome.

Overexpression of dominant inactive GDP bound form of RAB-5 (S33N) DN does not affect NLP-21-YFP levels at the synapse (Fig. 50), suggesting that changes induced in unc-108 mutants are specific for the activated form of RAB-5.
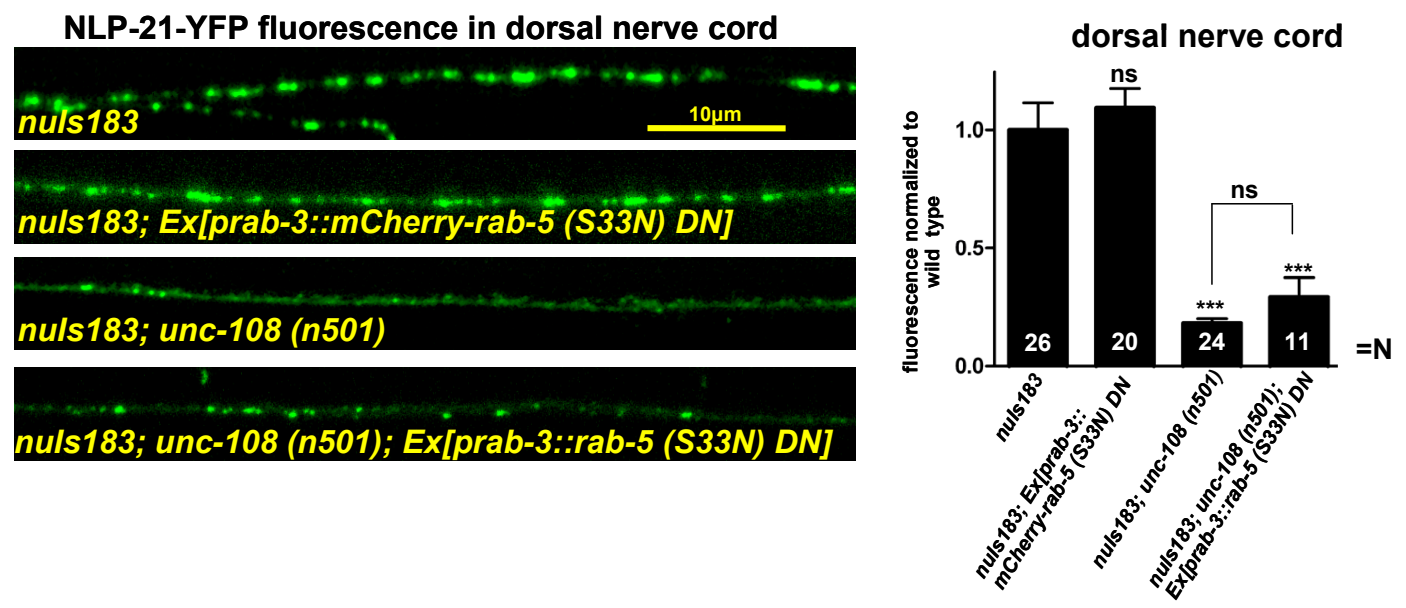

Figure 50. The overexpression of dominant inactive GDP bound RAB-5 (S33N) DN has no effect on neuropeptide levels at the synapses of unc-108 mutants.

mCherry tagged dominant inactive GDP bound form of RAB-5 was expressed panneuronally in nuls183 strain which stably expresses neuropeptide NLP-21 tagged to YFP. The

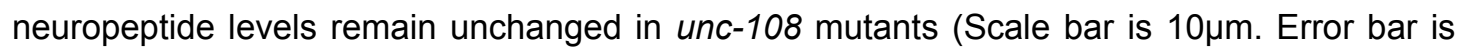
SEM. Number of animals is indicated in the graph bars, ${ }^{* * *} p<0.005$, Student's T-test). 


\subsection{RAB-2 mutations affect GGA localization at the Golgi/endosomal interface}

The iDCV membrane remodeling occurs via formation of the clathrin coated vesicles, and retrieval of the unnecessary proteins to the endosomal system. Overexpression or RNAi of GGA proteins inhibits clathrin coated vesicle budding from iDCVs (Kakhlon et al., 2006). GGAs are clathrin adaptor proteins involved in protein sorting from trans Golgi network.
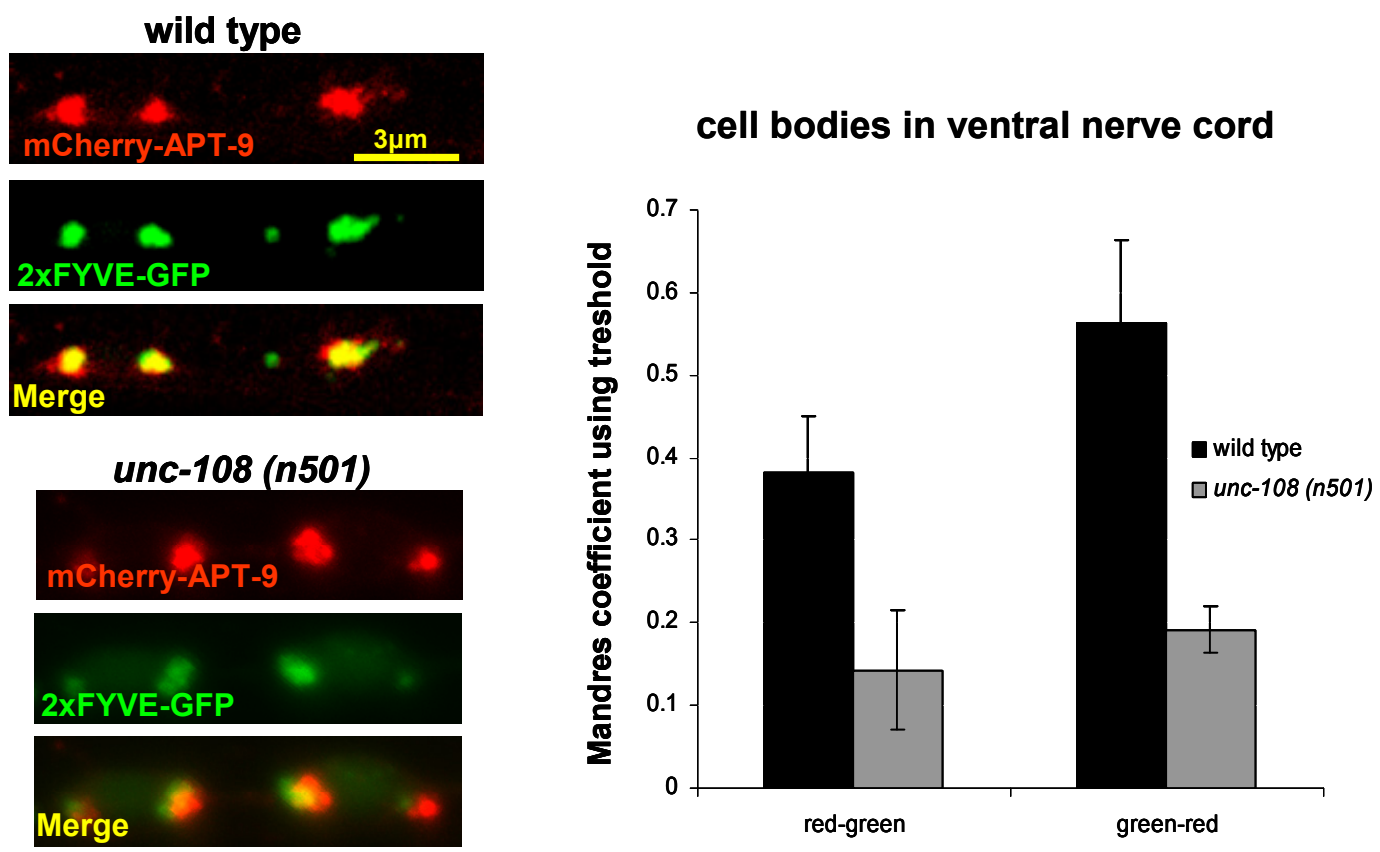

Figure 51. GGA protein APT-9, responsible for clathrin coated vesicle formation, mislocalizes in unc-108 mutants.

Left- representative images of mCherry-APT-9 and FYVE domain of EEA-1 tagged to GFP. Right - quantification of colocalization. In wild type most of the APT-9 colocalizes with early endosomal marker. In unc-108 mutants however, this colocalization is not as profound. Scale bar is $3 \mu \mathrm{m}$. (Error bar is SEM. $\mathrm{N}=10$ animals per strain). 
While in mammals three isoforms of GGA exist, C. elegans has only one, APT-9. If the function of APT-9 is conserved in C. elegans, APT-9 would be a good marker for the iDCV intermediate compartment where the membrane remodeling takes place. As seen on figure 50, in wild type most of the mCherry tagged APT-9 protein overlaps with early endosomal marker, FYVE domain of EEA-1. In unc-108 mutants, they colocalize less suggesting impairment in this step of the maturation pathway (Fig. 51).

\subsection{RAB-2 cooperates with its effector RIC-19 during DCV biogenesis}

Rab GTPases act via their effectors which they recruit in activated, GTP bound form. Recently it has been shown that in insulin secreting insulinoma cells the diabetes autoantigen ICA69 localizes to the Golgi complex and binds to activated Rab2 (Buffa et al., 2008; Spitzenberger et al., 2003). ICA69 is enriched in pancreatic $\beta$-cells and neurons. Its C. elegans ortholog, RIC-19, has been shown to be exclusively expressed in neurons (Pilon et al., 2000). Due to their similarities, we reasoned that RIC-19 acts as an effector of RAB-2 in DCV maturation. Both ICA69 and RIC-19 contain a BAR domain, a banana shaped structure shown to bind to and stabilize the membrane curvatures (Fig. 52). Similar to unc-108 mutants, ric-19 (ok833) deletion mutants display reduced locomotion (Fig. 53A). This movement defect can be rescued by expressing ric-19 specifically in neurons, suggesting that RIC-19 might be required for efficient signaling at the neuromuscular junction. In agreement 
with this, ric-19 (ok833) mutants are resistant to aldicarb. The aldicarbresistance of ric-19 is slightly weaker than that of unc-108 (n501) mutants and can also be completely rescued by neuronal expression of ric-19 (Fig. 53B).

A

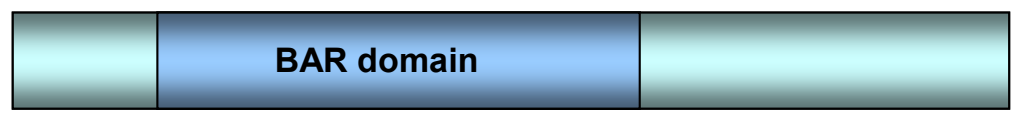

aa $52-250$

B

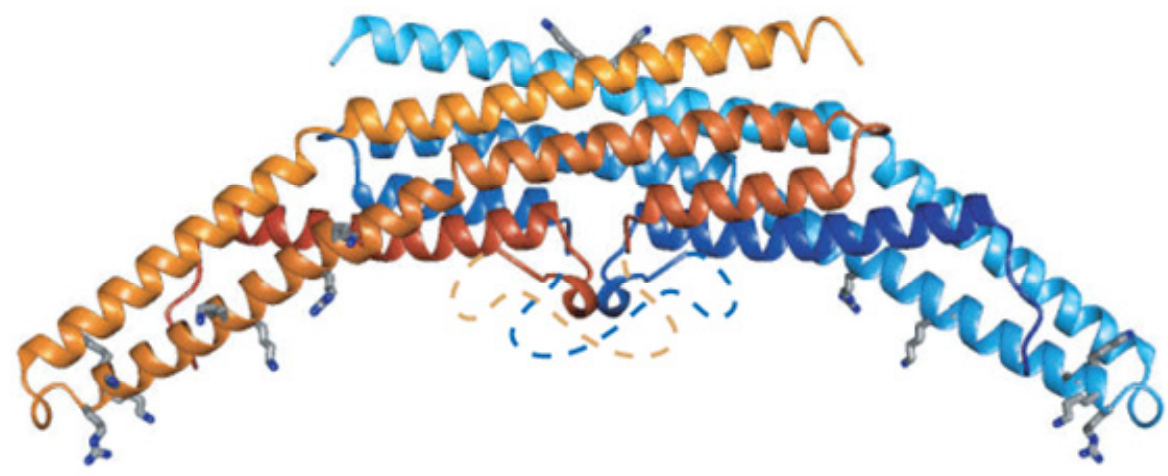

Figure 52. RIC-19 is a BAR domain containing protein.

(A) RIC-19 is an ortholog of mammalian ICA69 and it contains a BAR domain, which dimerizes and is known to recognize and bind to curved membranes found on vesicles and tubular carriers

(B) Structure of BAR domain. BAR domains are banana shaped domains that bind to and stabilize membrane curvatures. (Adapted from Gallop et al., 2006). 
A

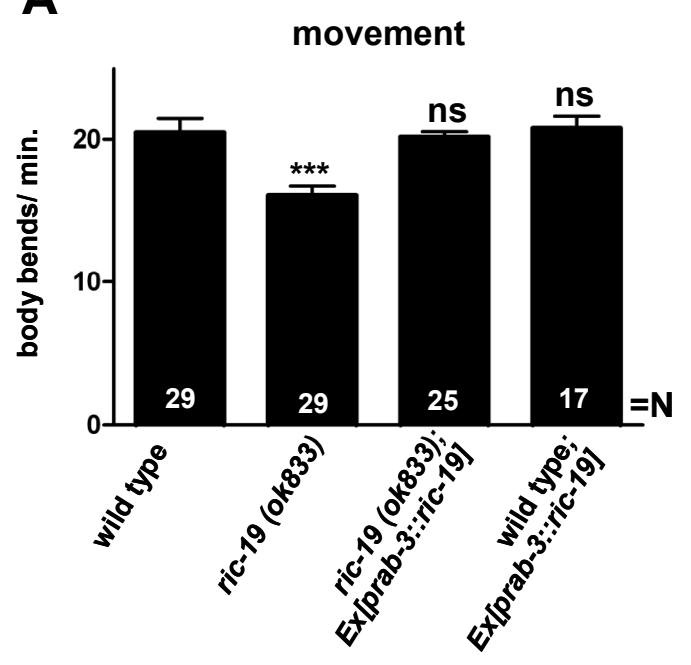

B aldicarb sensitivity

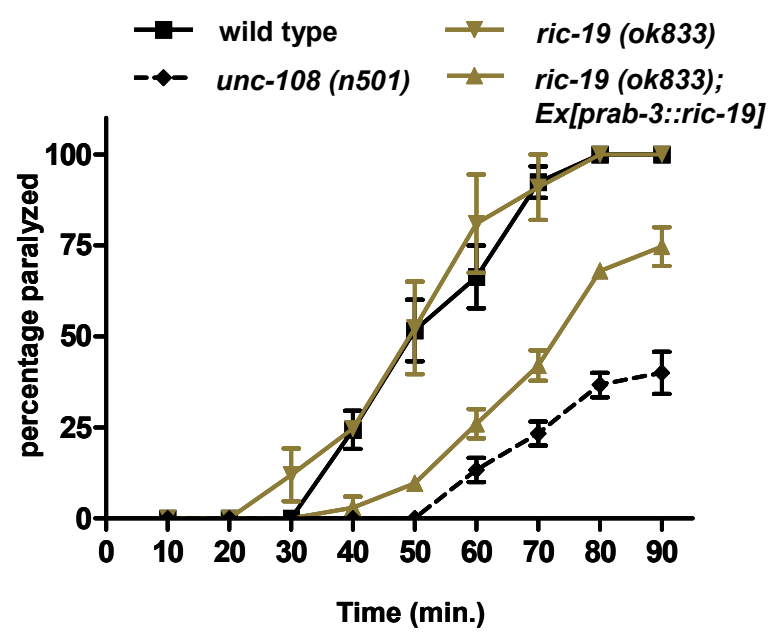

Figure 53. ric-19 mutants show similar phenotypes to unc-108 mutants.

(A) ric-19 mutants show movement defects, which are rescued by expression of ric-19 specifically in the nervous system. However, the pan-neuronal overexpression of RIC-19 in wild type background does not cause any phenotype. Knock down of RIC-19 in unc-108 has no effect on movement. ( ${ }^{* * *} p<0.005$, Student's t-test, error bar=SEM, $N$ is indicated).

(B) ric-19 (ok833) mutant animals are resistant to acetylcholine esterase inhibitor aldicarb similarly to unc-108 (n501). Aldicarb resistance can be rescued by neuronal expression of ric$19($ error bar $=$ SEM, $\mathrm{N}=30)$.

To exclude changes in synaptic morphology or SV pools in ric-19 mutants, we analyzed the synaptic ultrastructure by EM. Both the morphology and the number and distribution of SVs were similar to wild type, as seen for unc-108 mutants (Fig. 54 and 55 and Table 7). Consistent with this observation, the evoked release in ric-19 mutants was not significantly reduced (Fig. 56), suggesting that SV function is normal. 


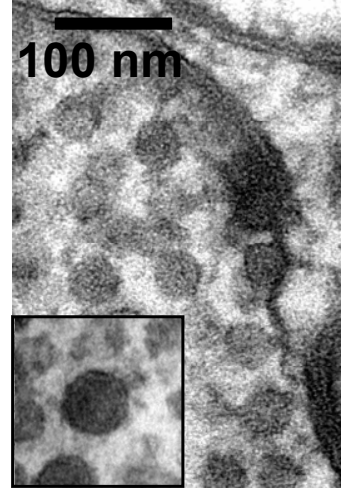

wild type

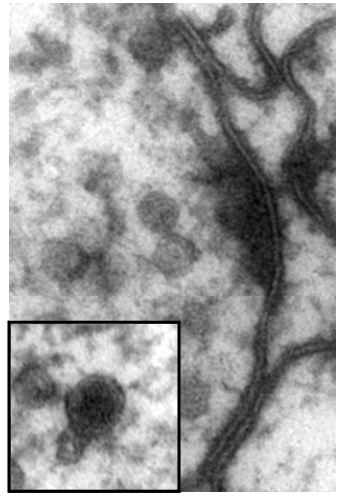

ric-19 (ok833)
Figure 54. The synaptic morphology in ric-19 mutants is similar to wild type.

The morphology of motorneuron synapses in the dorsal nerve cord in different $C$. elegans strains are shown in $40 \mathrm{~nm}$ HPF EM cross sections, with electron micrographs of single DCV shown in inlet. (Courtesy of Jan Hegermann).

\begin{tabular}{lcccccc}
\hline & $\begin{array}{c}\text { Number of } \\
\text { profiles/animals } \\
\text { analyzed }\end{array}$ & $\begin{array}{c}\text { Average } \\
\text { synaptic }\end{array}$ & $\begin{array}{c}\text { Average } \\
\text { area }\left(\mu \mathrm{m}^{2}\right)\end{array}$ & $\begin{array}{c}\text { diameter } \\
\text { profile }\end{array}$ & $\begin{array}{c}\text { Average } \\
\text { of SVs } \\
(\mathrm{nm})\end{array}$ & $\begin{array}{c}\text { DCV/profile } \\
\text { diameter of } \\
\text { DCVs }(\mathrm{nm})\end{array}$ \\
\hline wild type & $30 / 5$ & $0.25 \pm 0.02$ & $34.9 \pm 2.4$ & $30,3 \pm 0.7$ & $2.0 \pm 0.2$ & $44,8 \pm 1.0$ \\
unc-108 (n501) & $31 / 4$ & $0.23 \pm 0.03$ & $38.7 \pm 1.7$ & $27,7 \pm 0.5$ & $2.3 \pm 0.3 \mathrm{~ns}$ & $49,4 \pm 2.3 *$ \\
ric-19 (ok833) & $32 / 4$ & $0.22 \pm 0.01$ & $33.1 \pm 1.3$ & $27,2 \pm 0.6$ & $2,7 \pm 0.4 \mathrm{~ns}$ & $42,0 \pm 2.0 \mathrm{~ns}$ \\
rab-3 (js49) & $13 / 3$ & $0.22 \pm 0.02$ & $37.7 \pm 3.4$ & $27,8 \pm 0.6$ & $3.2 \pm 0.5 *$ & $41,7 \pm 1.1$ * \\
\hline
\end{tabular}

Table 8. Statistics on SV and DCV numbers and diameters as determined by HPF EM, the average synaptic area, number of synapses and independent animals analyzed are indicated. The DCV statistics of ric-19 mutants (highlighted in orange) are compared to wild type (Student's T-test). (Courtesy of Jan Hegermann and Christian Olendrowitz) 


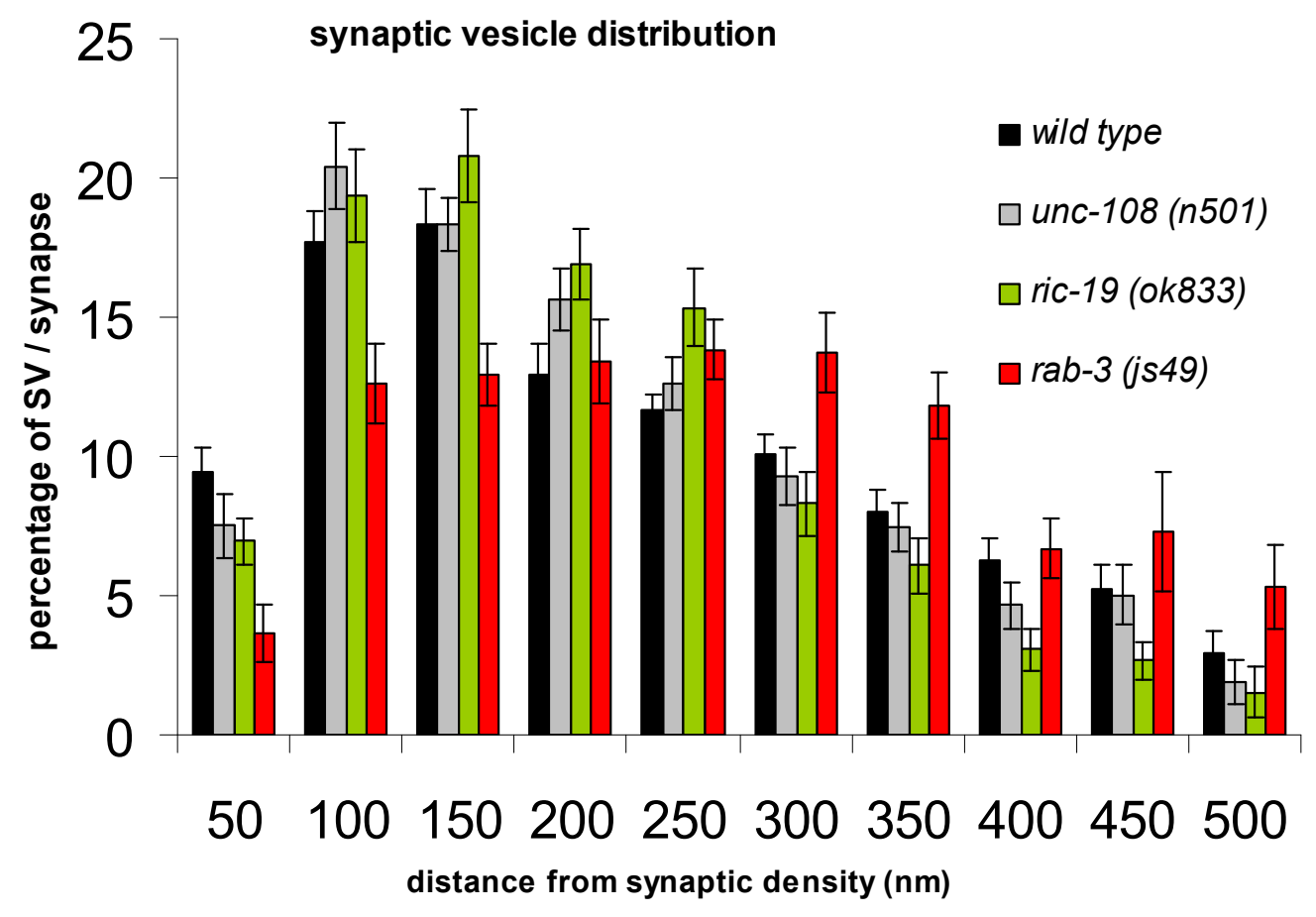

Figure 55. The SV distribution is not changed in ric-19 mutants.

The SV distribution relative to the presynaptic density at release sites was analyzed for unc108 and ric-19 mutants. SV distribution in rab-3 mutants, exhibiting SV tethering defects, is shown as control. ric-19 data is not significantly different compared to wild type (one way ANOVA with Dunett posttest). (Courtesy of Jan Hegermann and Christian Olendrowitz).

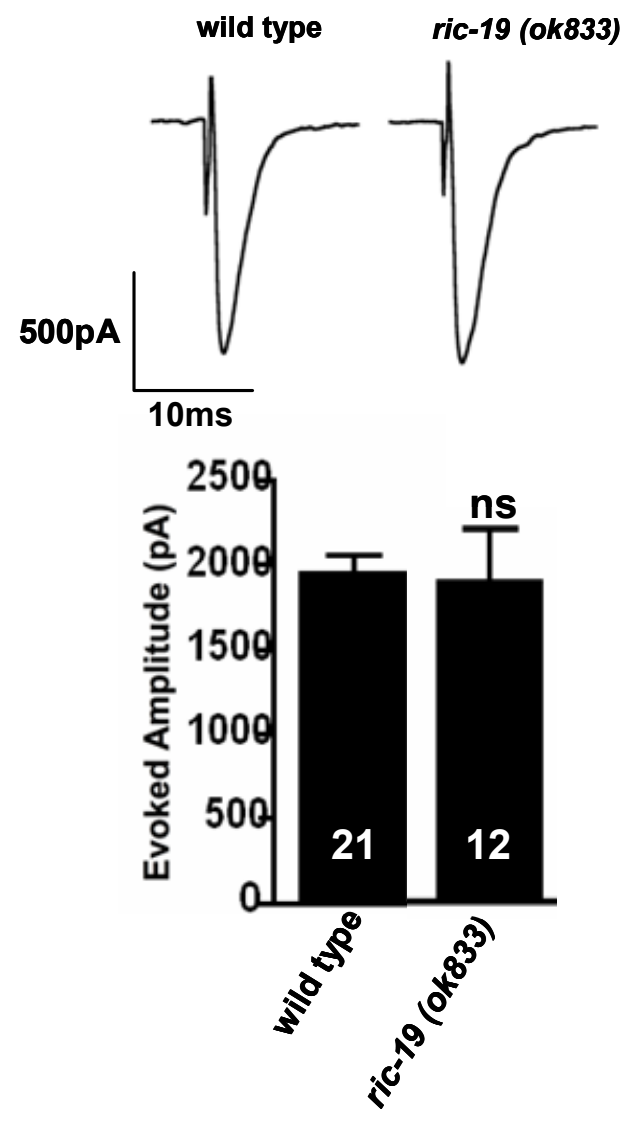

Figure 56. ric-19 mutants have do defects in SV release at the neuromuscular junction.

The evoked release of SVs at the neuromuscular junction is similar between the wild type and ric-19 mutants (Number of animals analyzed is indicated in the graph bars, error bar is SEM). 
We therefore tested whether ric-19 mutants have reduced NLP-21 derived YFP cargo in their axons similar to unc-108 mutants. We found that ric-19 (ok833) mutants have a $50 \%$ reduction in the NLP-21-YFP fluorescence in motorneuron axons. This is accompanied by a corresponding $50 \%$ reduction of NLP-21-YFP secretion as judged by coelomocyte fluorescence. Both defects were completely rescued by neuronal expression of ric-19 (Fig. 57 and 58). This strongly suggests that RIC-19 and RAB-2 function in the same pathway during DCV maturation. Consequently, the appearance of large NLP21-YFP filled vesicular structures can also be seen in the cell bodies of ric-19 mutant motorneurons in addition to the disappearance of smaller vesicular structures (Fig. 59). Thus ric-19 null mutants behave similar to the dominant unc-108/rab-2 alleles in terms of aldicarb sensitivity and DCV defects.

dorsal nerve cord
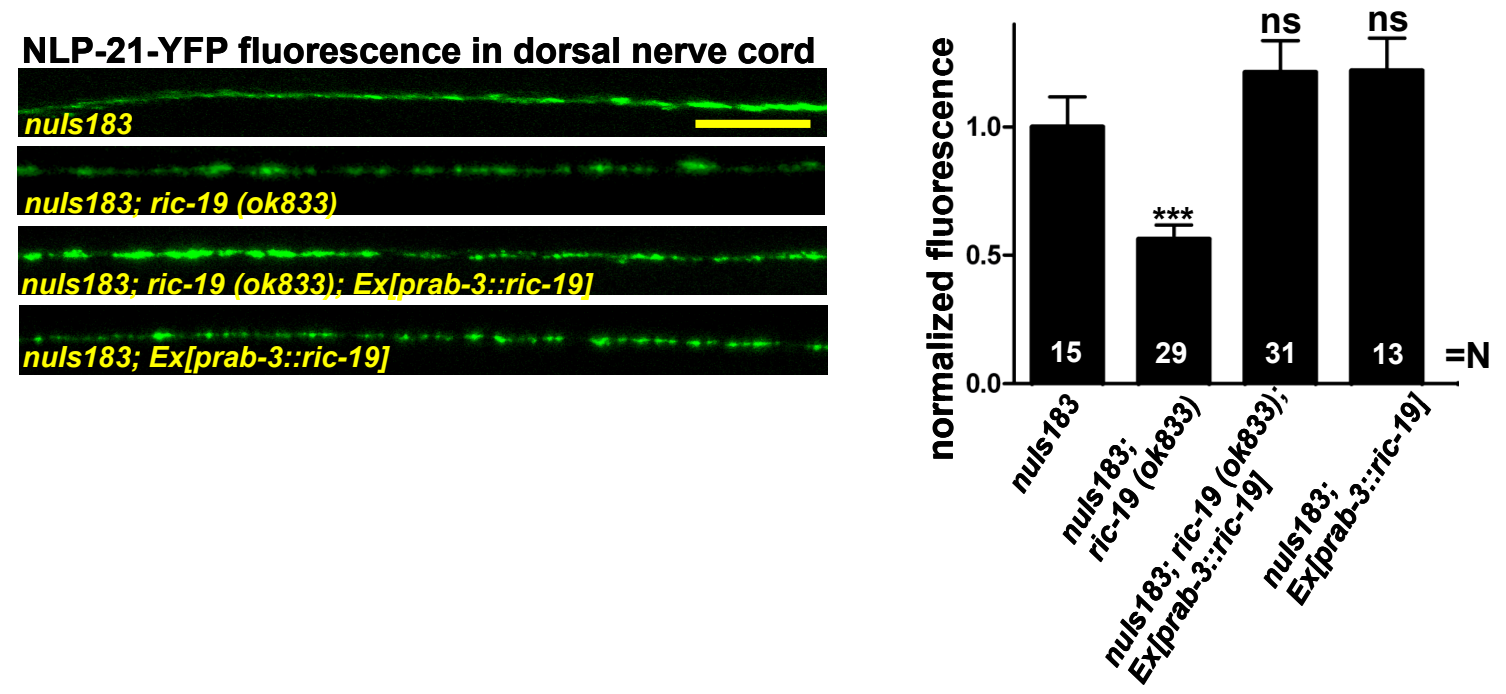

Figure 57. ric-19 mutants have strongly reduced soluble cargo in DCVs at the synapses.

ric-19 deletion mutants show decreased amount of NLP-21-YFP fluorescence at synapses to the levels similar to unc-108 mutants. Defect observed in ric-19 mutants can be rescued by 
neuronal expression of ric-19. Pan-neuronal overexpression of RIC-19 in NLP-21-YFP expressing integrated array nuls183, does not affect NLP-21 levels at the synapse. (Scale bar $10 \mu \mathrm{m},{ }^{* * *} \mathrm{p}<0.005,{ }^{* *} \mathrm{p}<0.01$, Student's t-test, error bar=SEM; $N$ is indicated in the graph bars).
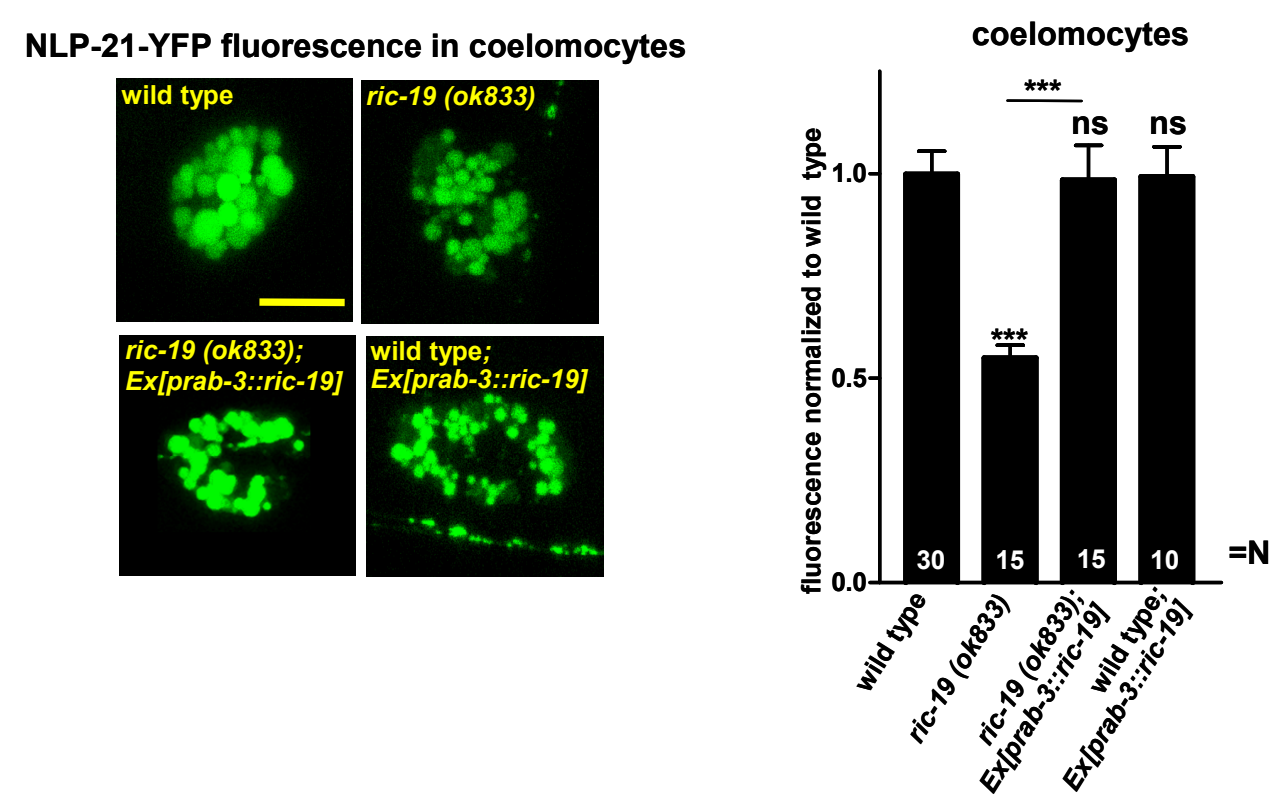

Figure 58. NLP-21-YFP secretion is reduced in ric-19 mutants.

The secretion of NLP-21-YFP is decreased in ric-19 mutants and is rescued by pan-neuronal expression of ric-19. (Scale bar $5 \mu \mathrm{m},{ }^{* * *} \mathrm{p}<0.005$, Student's t-test, error bar $=\mathrm{SEM}$; $\mathrm{N}$ is indicated in the graph bars). 
A

NLP-21-YFP in ventral nerve cord

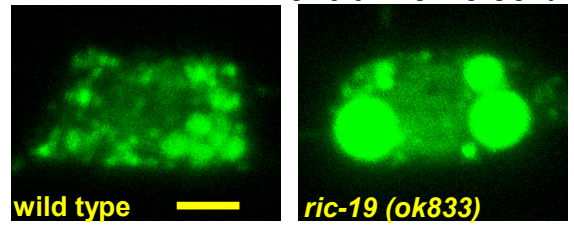

C

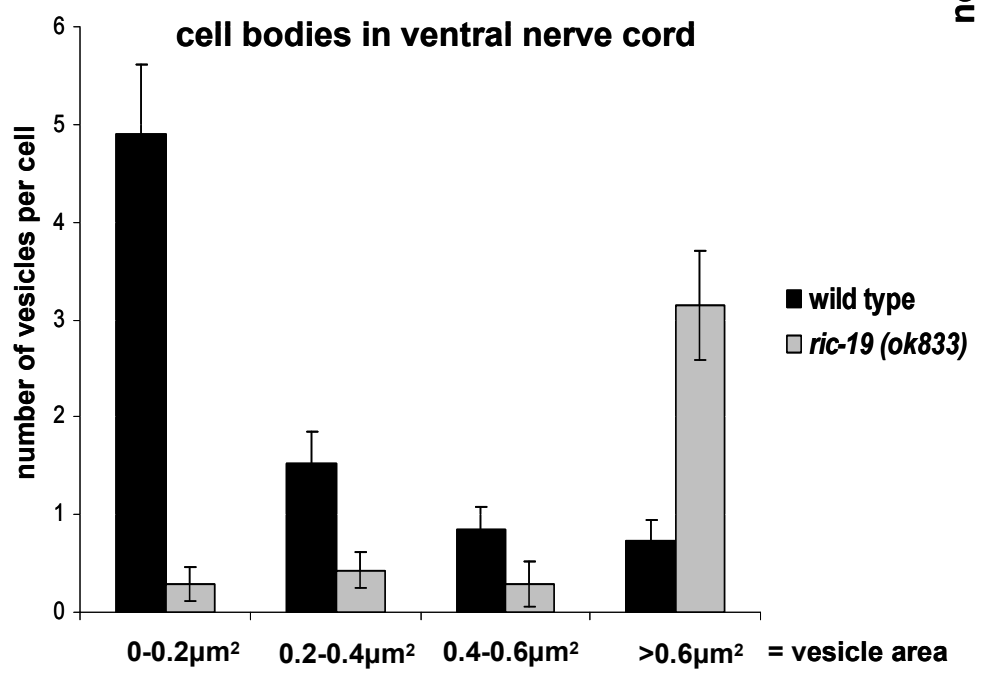

B

cell bodies

in the ventral nerve cord

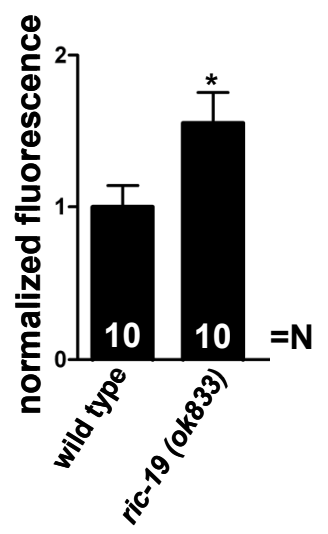

Figure 59. In ric-19 neuronal cell bodies, NLP-21-YFP positive vesicular structures are greater in size.

(A) NLP-21-YFP is expressed in cholinergic motorneurons from integrated array nuls183 and shown are representative images of neuronal cell bodies in the ventral nerve cord of wild type animals and ric-19 mutants.

(B) The amount of neuropeptide present in the neuronal cell bodies increased in ric-19 deletion strain (Scale bar $2 \mu \mathrm{m},{ }^{*} \mathrm{p}<0.05$, Student's T-test, error bar $=\mathrm{SEM}$, number of animals analyzed is indicated in the graph bars)

(C) NLP-21-YFP neuropeptide positive vesicles have different size distribution in ric-19 mutants compared to wild type (error bar = SEM, N=10). 

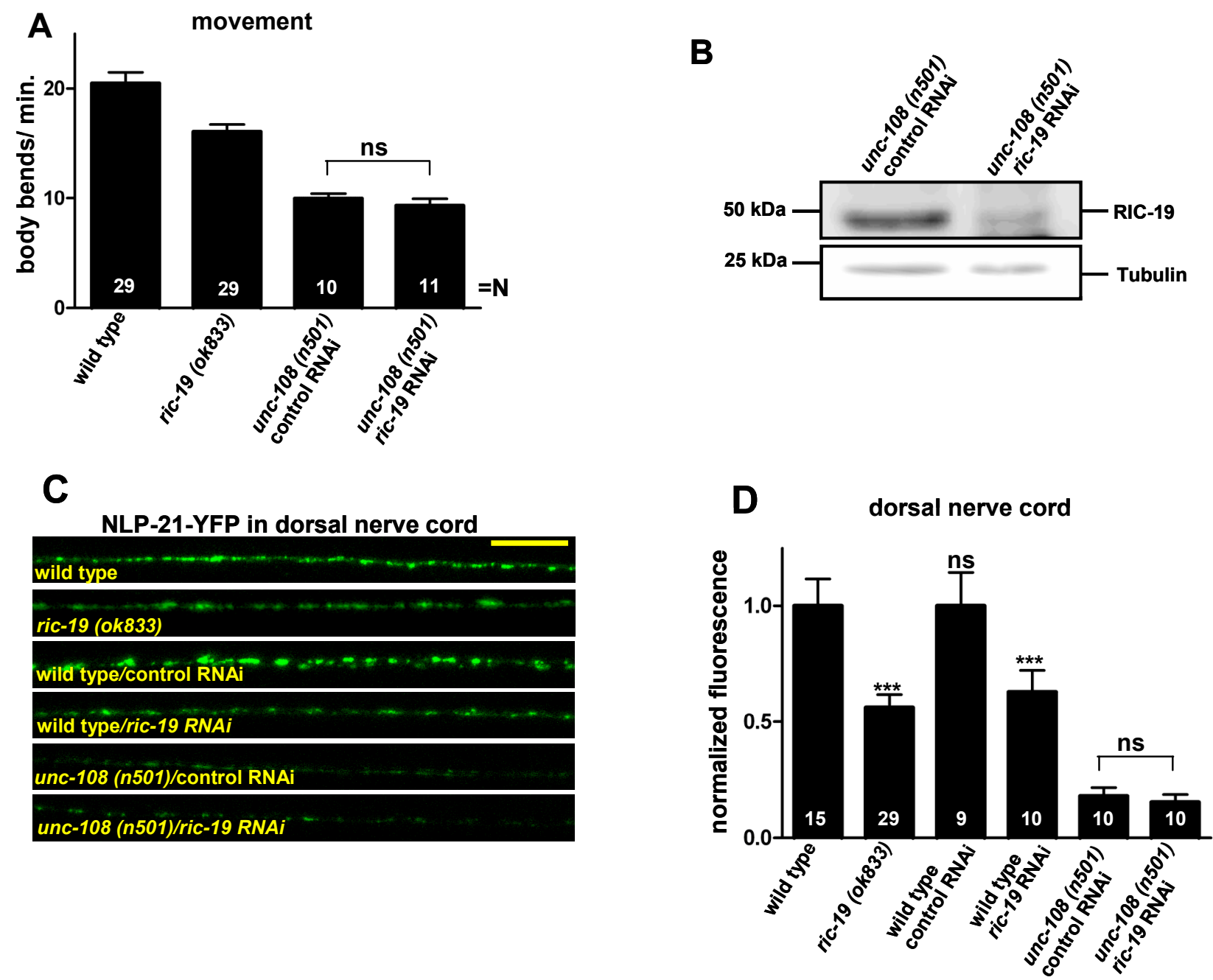

Figure 60. Knockdown of RIC-19 in unc-108 mutants does not change the phenotype.

(A) Knockdown of RIC-19 in unc-108 has no effect on movement. ${ }^{* * *} p<0.005$, Student's ttest, error bar=SEM, $\mathrm{N}$ is indicated).

(B) Western blot showing the knockdown of RIC-19 in unc-108 (n501) strain.

(C) and (D) Knock down of RIC-19 in wild type abolishes the neuropeptide level at the synapse to the ric-19 levels, but has no effect in unc-108 background. (Scale bar $10 \mu \mathrm{m}$, ${ }^{* * *} p<0.005,{ }^{* *} p<0.01$, Student's t-test, error bar=SEM; $N$ is indicated in the graph bars).

Unfortunately, ric-19 and unc-108 are both located on chromosome I and only separated by $45 \mathrm{~kb}$, so it was impossible to generate the ric-19;unc-108 double mutants. Therefore, we used RNA interference to show that unc108/rab-2 and ric-19 are in the same pathway required for DCV maturation. In 
agreement with this, RNAi mediated knock down of RIC-19 by bacterial feeding in unc-108 mutants did not enhance the movement defects (Fig. 60A) nor the loss of NLP-21-YFP fluorescence in the dorsal cord axons (Fig. 60C and D). The ric-19 RNAi was clearly functional since we could reproduce the loss of NLP-21-YFP fluorescence in wild type animals by ric-19 RNAi and also the down-regulation of endogenous RIC-19 as shown by Western blotting (Fig. 60B). This highly suggests that RAB-2 affects DCV maturation via RIC19. Pan-neuronal expression of RIC-19 in wild type animals did not result in any detectable locomotory defects, loss of NLP-21 derived YFP from DCVs or defects in DCV release as measured by the uptake of secreted YFP by coelomocytes (Fig. 58 and 59).

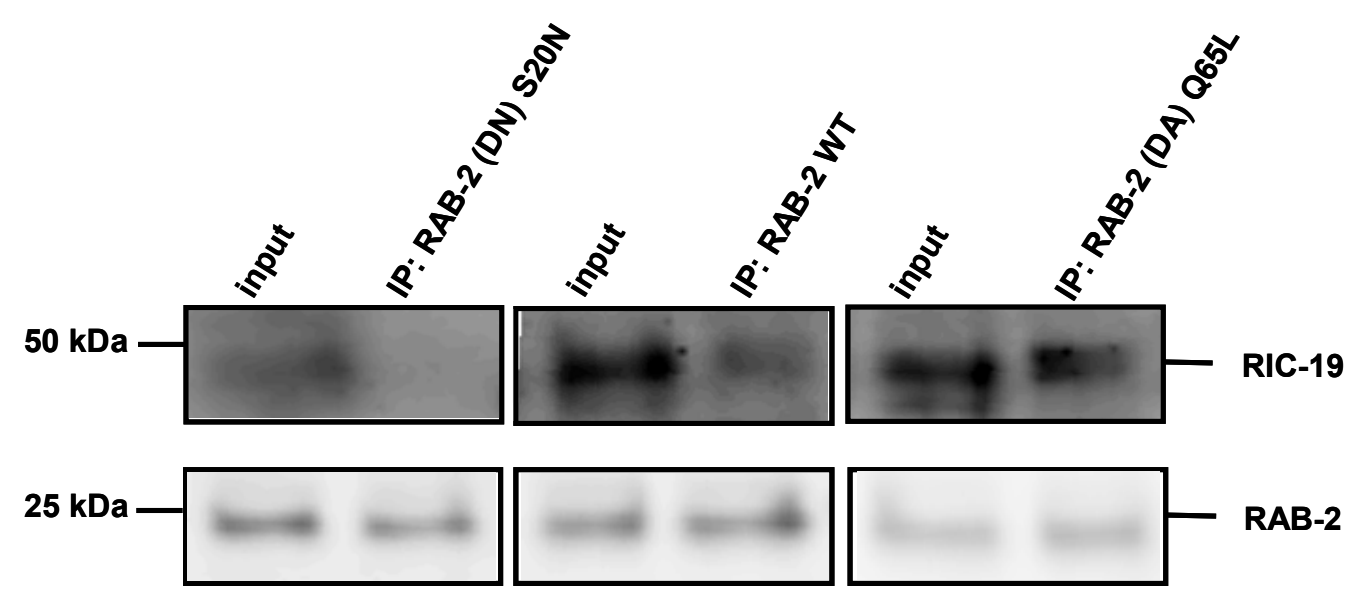

Figure 61. RIC-19 coimmunoprecipitates with dominant active GTP bound RAB-2.

When expressed in HeLa cells, RIC-19 co-immuno-precipitates with wild type RAB-2 and its GTP bound form RAB-2(Q65L)DA, but not its inactive, GDP bound form, RAB-2(S20N)DN. The amount of affinity purified RAB- 2 is shown below as a control.

Based on the fact that both molecules are in same pathway and the similarity of phenotypes, we wanted to determine whether RIC-19 is a direct effector of RAB-2 in C. elegans. To test this, we coexpressed RIC-19 with either the wild 
type, the dominant active GTP bound and dominant inactive GDP bound HA tagged RAB-2 in HeLa cells. Subsequently, HA-RAB-2 was immunoprecipitated by using anti HA antibodies and the copurification of RIC-19 was analyzed by Western blotting. As shown in Fig. 61, RIC-19 can be specifically affinity-purified with RAB-2 DA and to a lesser extent wt RAB-2, but not with the inactive form RAB-2 DN. These results suggest that RIC-19 is an effector of RAB-2 and that both proteins are required for proper DCV maturation.

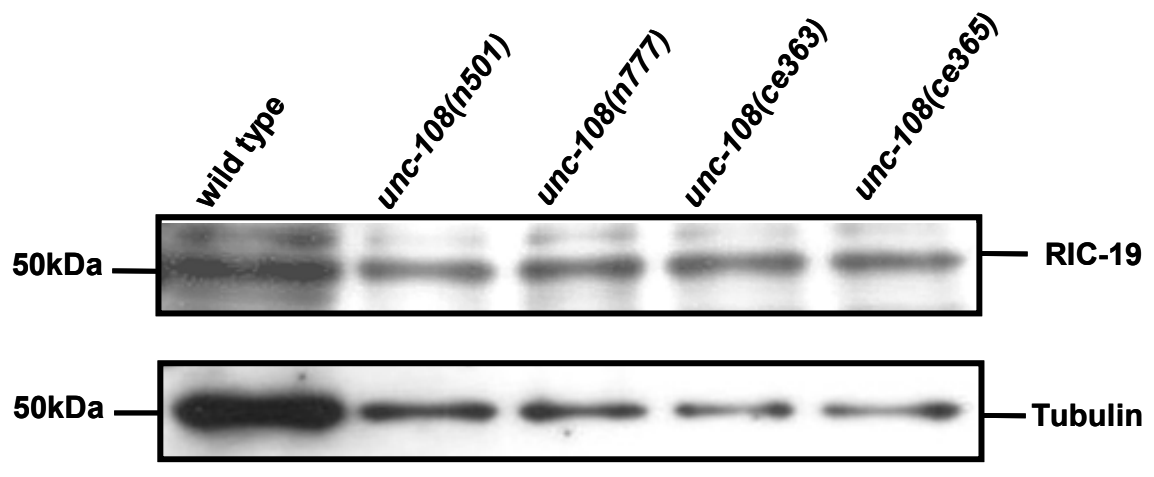

Figure 62. The RIC-19 protein levels remain the same in unc-108 mutants.

The expression of RIC-19 is not altered in unc-108 mutants as judged by Western blots with RIC-19 antibody detecting the endogenous protein in lysates from different unc-108 strains.

Since RAB-2 and RIC-19 are binding to each other, we wondered whether the stability of RIC-19 would be affected by mutations in unc-108. However, the endogenous RIC-19 protein levels are not affected in unc-108/rab-2 mutant animals as judged by Western blots with RIC-19 antibodies (Fig. 62). RIC-19 showed mostly cytoplasmatic, diffuse localization, with no discrete puncta observed in the cytoplasm. Interestingly, expression of dominant active GTP bound form of RAB-2 was associated with a stronger localization of RIC-19 at the Golgi and a decrease in diffuse cytoplasmatic stain. However, the 
expression of dominant inactive GDP bound form of RAB-2, did not change the localization pattern of RIC-19. This suggests that RAB-2 recruits RIC-19 at Golgi (Fig. 63) While wild type RAB-2 expressing neurons show a large pool of diffusely localized RIC-19-mYFP, most of RIC-19-mYFP is recruited to discrete puncta by the dominant active GTP bound RAB-2 (Q65L) DA.

\section{A}
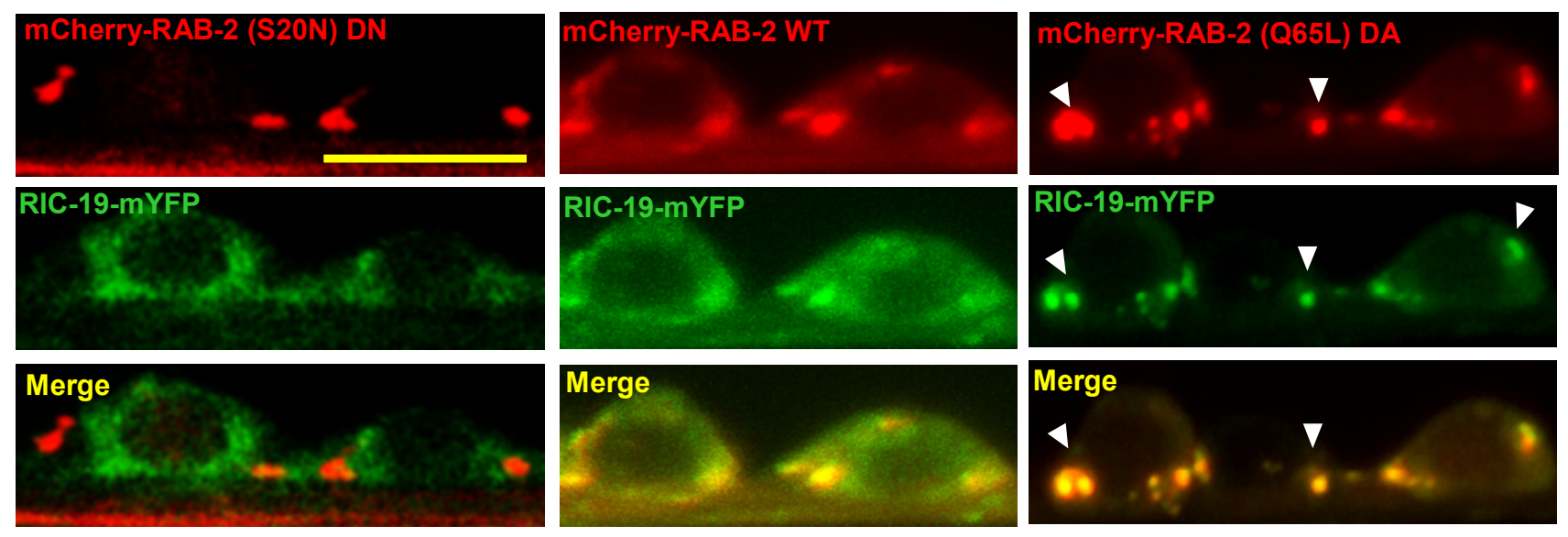

B

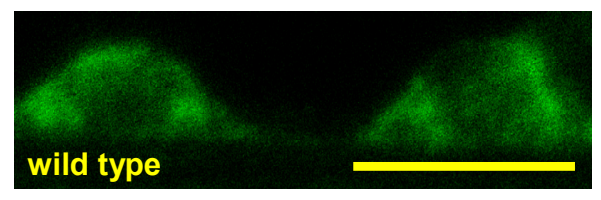

RIC-19-mYFP in ventral nerve cord neurons
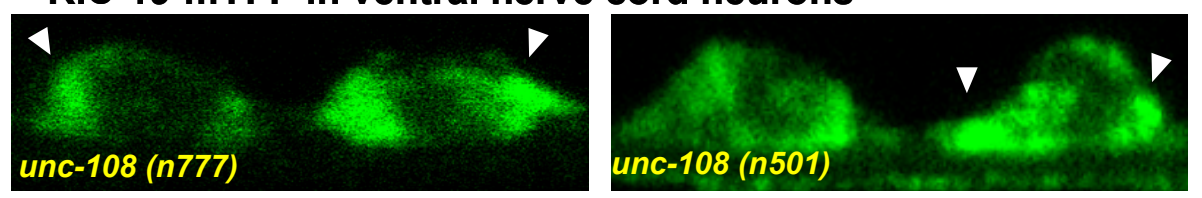

Figure 63. RAB-2 GTP bound form recruits RIC-19 to the Golgi.

(A) RIC-19 localization is mostly cytoplasmatic in cells expressing the dominant inactive, GDP bound RAB-2, RAB-2(S20N) DN. RIC-19 is recruited to Golgi membranes by expression of either wild type RAB-2 or to greater extend by the dominant active GTP bound RAB-2 (Q65L)

DA form. Note that by expression of RAB-2(Q65L) DA most of the diffuse cytoplasmatic RIC$19-m Y F P$ is localized to discrete Golgi puncta. Scale bar $4 \mu m$.

(B) As compared to wild type motorneurons, RIC-19-mYFP shows increased Golgi recruitment in the dominant unc-108 mutants (marked by arrowheads). Scale bar $4 \mu \mathrm{m}$. 
Consistent with this possibility, expressing RIC-19 in the dominant unc-108 mutants (n777) and (n501) produced a RIC-19 localization pattern similar to that seen by co-expressing RIC-19 with dominant active GTP bound RAB-2 (Q65L) DA. In both dominant mutants, the localization of RIC-19-mYFP was more confined in neuronal cell bodies as compared to wild type (Fig. 63), more strongly in $n 501$ than in the $n 777$ mutant. Together, our results suggest that RAB-2 and its effector RIC-19 cooperate during DCV maturation to retain specific cargo in maturing DCVs and prevent its loss to the endosomallysosomal system.

\subsection{Constitutively active RAB-5 blocks DCV secretion}

We showed that overexpression of dominant active RAB-5 rescues the NLP21 derived YFP levels at the unc-108 synapses. During the course of our experiment, we realized that the expression of dominant active RAB-5 although rescuing the axonal NLP-21 derived YFP levels, led to a block of DCV secretion (Fig. 64). In agreement with this, neuronal expression of dominant active RAB-5 decreased the DCV secretion by $80 \%$ (Fig. 65). Since dominant active RAB-5 is only expressed in neurons, it could not affect the endocytosis of coelomocytes. 

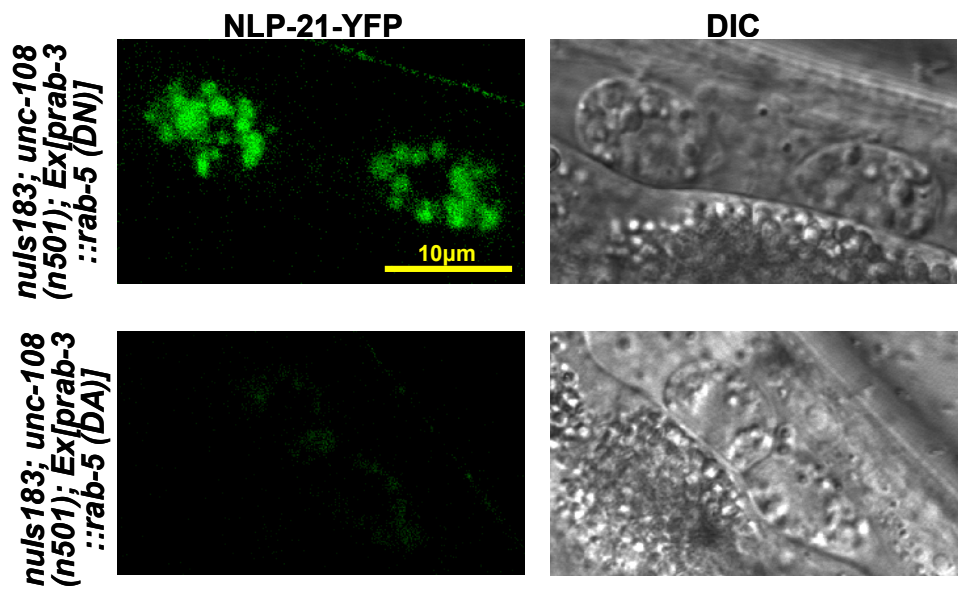

Figure 64. Neuronal expression of dominant active RAB-5 blocks the DCV secretion from unc-108 neurons.

NLP-21-YFP was expressed in cholinergic motorneurons in the strain nuls183. The expression of dominant active but not dominant negative RAB- 5 blocks the NLP-21 derived YFP secretion in unc-108 mutant background (Scale bar is $10 \mu \mathrm{m}$ ).

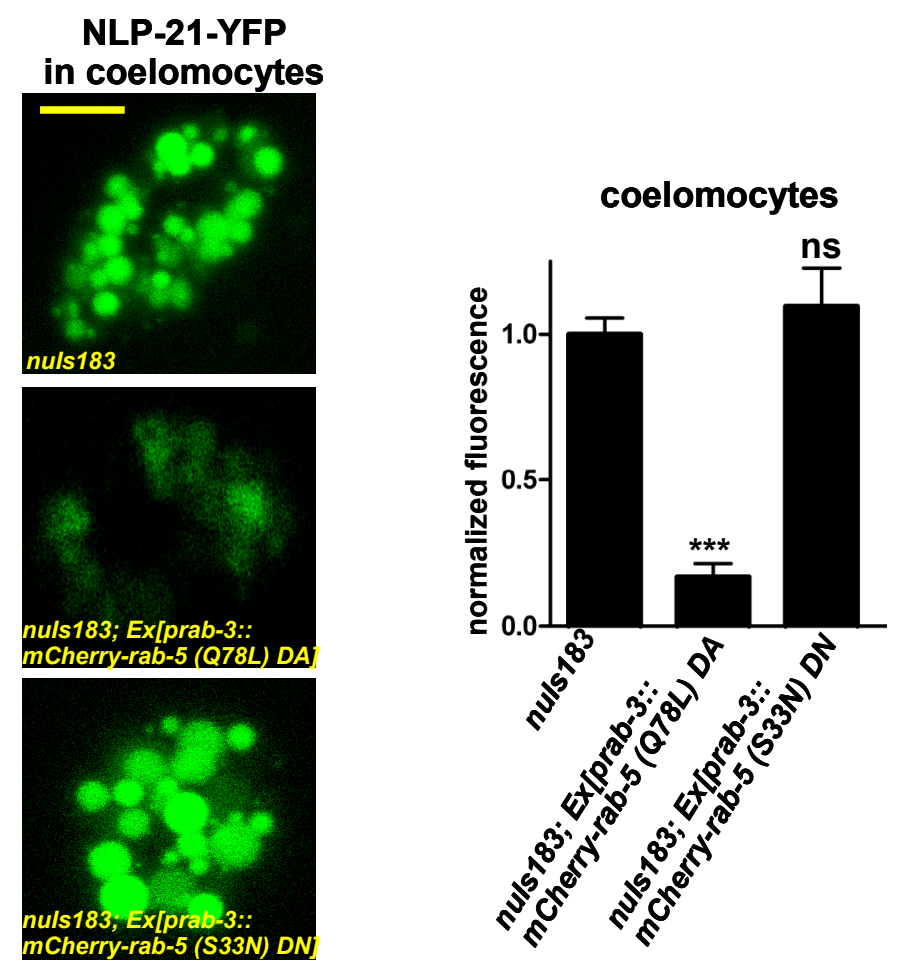

Figure 65. The overexpression of dominant active GTP bound RAB-5 blocks the secretion of DCVs.

mCherry tagged dominant active GTP bound form of RAB- 5 was expressed panneuronally in nuls183 strain which stably expresses neuropeptide NLP-21 tagged to YFP. Once secreted at 
the synapse, NLP-21-YFP is taken up by coelomocytes. The overexpression of RAB-5 reduces the fluorescence in coelomocytes, thus reduces the secretion of the peptide at the synapses. (Scale bar is $5 \mu \mathrm{m}$. Error bar is SEM, number of animals analyzed is indicated in the graph bars, ${ }^{* * *} \mathrm{p}<0.005$, Student's T-test).

Accordingly, the overexpression of dominant active RAB-5 in neurons, leads to movement defects and aldicarb resistance (Fig. 66). This is in agreement with blockage of DCV signaling.

A

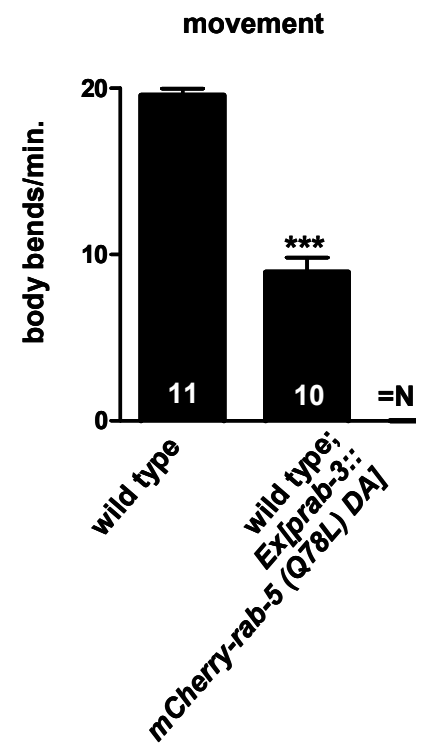

B

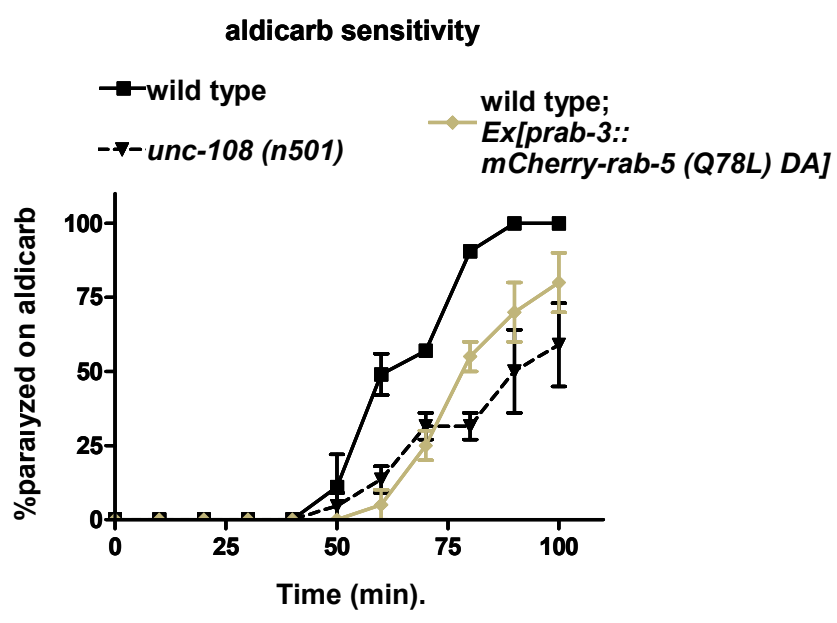

Figure 66. Overexpression of dominant active RAB-5 in neurons leads to movement defects and aldicarb resistance.

(A) Overexpression of dominant active RAB-5 in neurons decreases movement by $50 \%$ compared to wild type (Number of animals analyzed is indicated in the graph bars, error bar is SEM, ${ }^{* * *} p<0.005$, Student's T-test)

(B) Overexpression of dominant active RAB-5 in neurons leads to aldicarb resistance similar to the one of unc-108 ( $\mathrm{n} 501)$ mutants ( $\mathrm{N}=30$ animals per strain, error bar is $\mathrm{SEM}$ ). 
Surprisingly, expression of dominant active GTP bound RAB-5 (Q78L) DA induces formation big vesicular structures in unc-108 synapses, but not in the wild type (Fig. 67). These could either present the early endosomes at the synapses or abnormal either synaptic or DCV fusions. We were not able to identify the identity of these vesicular structures, since the markers are not available: EEA-1 is not present at the synaptic early endosomes and RAB-3 and SNB-1 are present on both synaptic and DCVs.
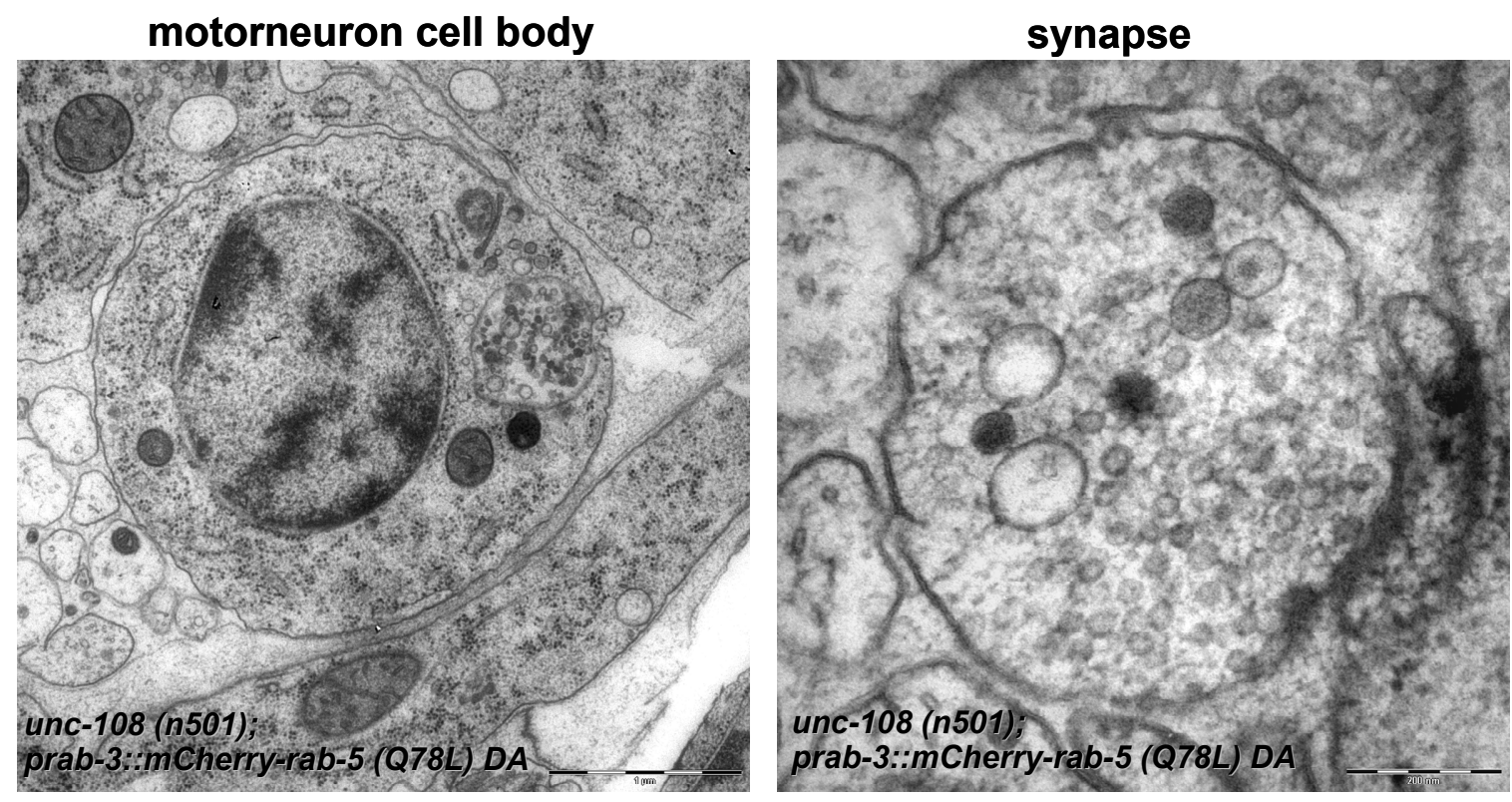

Figure 67. Expression of dominant active GTP bound form of RAB-5 induces formation of big vesicular structures at unc-108 (n501) synapses.

HPF EM cross sections of motorneuron cell bodies and synapse of unc-108 (n501) expressing dominant active GTP bound RAB-5 (Q78L) DA.

To further investigate this, we looked at the SV marker RAB-3 fused to YFP in the wild type worms expressing dominant active GTP bound RAB-5 (Q78L) DA. In the wild type background, overexpression of dominant active form of 
RAB-5 did not induce any changes in total number of fluorescence, nor synapse spacing or synaptic size (Fig. 68 and 69).
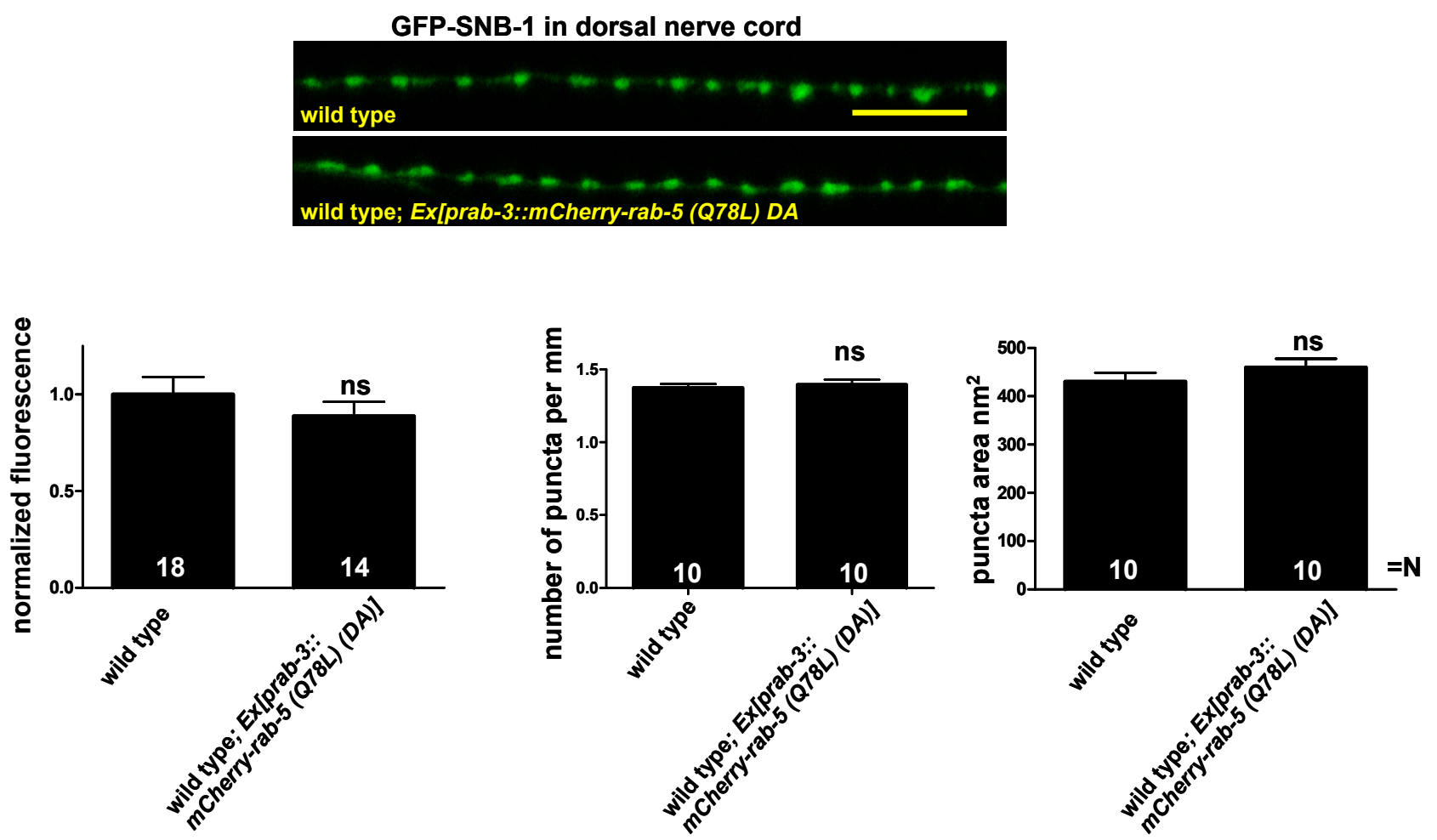

Figure 68. Overexpression of dominant active GTP bound form of RAB-5 does not induce synaptic morphology changes in wild type.

Dominant active GTP bound RAB-5 (Q78L) DA was expressed in strain expressing SNB-1GFP in cholinergic motorneurons. The synapse morphology appears to be similar between wild type and wild type expressing dominant active RAB-5, as judged by SNB-1-GFP puncta number, size and average fluorescence. (Scale bar is $10 \mu \mathrm{m}$, error bar is SEM, number of animals analyzed is indicated in the graph bars). 


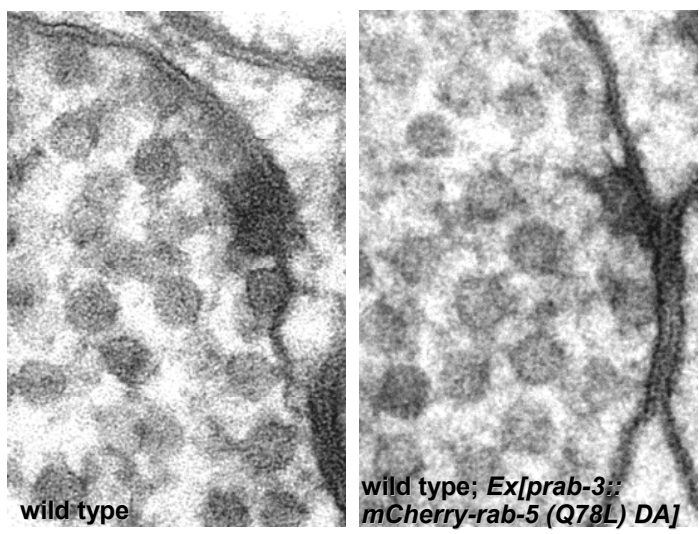

Figure 69. Expression of dominant active GTP bound form of RAB-5 does not change the morphology of wild type synapses.

HPF EM cross sections of synapses of wild type and wild type worms expressing dominant active GTP bound RAB-5 (Q78L) DA. (Courtesy of Jan Hegermann).

Furthermore, the distribution and the number of SVs does not change upon expression of dominant active RAB-5, as judged by the HPF EM data (Fig. 70 and Table 8). However, both SVs and DCVs appear to be smaller compared to wild type and DCV are more abundant. These data suggest that dominant active most likely affects DCV secretion specifically.

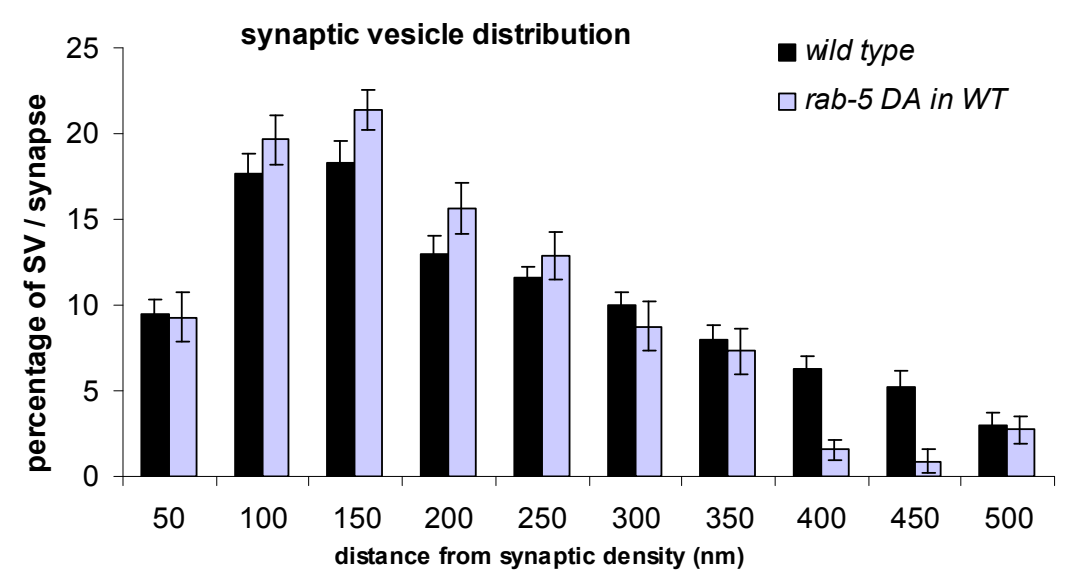

Figure 70. Expression of dominant active RAB-5 does not change the SV distribution in wild type animals.

The SV distribution relative to the presynaptic density at release sites was analyzed for wild type and wild type worms expressing dominant active GTP bound RAB-5 (Q78L) DA. (Courtesy of Jan Hegermann and Christian Olendrowitz) 


\begin{tabular}{|c|c|c|c|c|c|}
\hline Genotype & $\begin{array}{l}\text { Average } \\
\text { synaptic } \\
\text { area }(\mu \mathrm{m} 2)\end{array}$ & SV / profile & $\begin{array}{l}\text { Average } \\
\text { diameter of } \\
\mathrm{SV}(\mathrm{nm})\end{array}$ & $\begin{array}{l}\text { DCV I } \\
\text { profile }\end{array}$ & $\begin{array}{l}\text { Average } \\
\text { diameter of } \\
\mathrm{DCV}(\mathrm{nm})\end{array}$ \\
\hline wild type & $\begin{array}{l}0,25 \\
+/-0,02\end{array}$ & $\begin{array}{l}34,9 \\
+/-2,4\end{array}$ & $\begin{array}{l}30,3 \\
+/-0,7\end{array}$ & $\begin{array}{l}2,0 \\
+/-0,2\end{array}$ & $\begin{array}{l}44,8 \\
+/-1,0\end{array}$ \\
\hline rab-5 (Q78L) DA & $\begin{array}{l}0,20 \\
+/-0,01\end{array}$ & $\begin{array}{l}36,1 \\
+/-1,7\end{array}$ & $\begin{array}{l}26,4 \\
+/-1,0\end{array}$ & $\begin{array}{l}3,4 \\
+/-0,5\end{array}$ & $\begin{array}{l}36,4 \\
+/-0,9\end{array}$ \\
\hline
\end{tabular}

Table 9. SV and DCV statistics of dominant active RAB-5 expressing synapse.

Statistics on SV and DCV numbers and diameters as determined by HPF EM, the average synaptic area, number of synapses and independent animals analyzed are indicated. (Courtesy of Jan Hegermann and Christian Olendrowitz) 


\section{Discussion}

\subsection{Multiple roles for RAB-2 in membrane trafficking}

Rab GTPases have been shown to control almost all aspects of intracellular membrane trafficking (Fukuda, 2008; Zerial and McBride, 2001). In C. elegans and Drosophila, Rab2 is highly enriched in the nervous system (Chun et al., 2008; Mangahas et al., 2008; Zhang et al., 2007). In addition, C. elegans unc108/rab-2 mutants have been isolated based on their movement defects, indicative of changes in signaling at the neuromuscular junction (Park and Horvitz, 1986; Simmer et al., 2003). Interestingly, although differences exist between dominant and recessive unc-108/rab-2 alleles, they display similar paralyzed phenotypes (Chun et al., 2008; Lu et al., 2008; Mangahas et al., 2008). In addition to the movement defects, the recessive loss-of-function alleles are also defective in the removal of apoptotic cells and fluid phase endocytosis in the intestine as well as in the macrophage like coelomocytes (Lu et al., 2008; Mangahas et al., 2008). The recessive null allele nu415, which removes the last 66 amino acids of RAB-2, was also reported to affect glutamate receptor trafficking in a postendocytotic step (Chun et al., 2008). In contrast, animals carrying the dominant unc-108/rab-2 alleles $n 777$ or $n 501$ do not show clear defects in cell corps engulfment or in endocytosis. This suggests that RAB-2 might execute multiple cellular functions with specific requirements for different levels of RAB-2 activity. 
The dominant mutations RAB-2 (D122N) n777 and RAB-2 (S149F) n501 are positioned in the conserved G2 and G3 domains that stabilize GTP binding (Paduch et al., 2001). Using recombinant RAB-2 proteins, we show that biochemically, the $n 501$ and $n 777$ mutations also behave like the engineered dominant active GTP bound RAB-2 (Q65L) DA protein (Tisdale, 1999), lacking GTPase activity and/or exhibiting enhanced GTP affinity. Thus, they are expected to exist mostly in the GTP bound active form. In agreement with this, rab-2 (n501) and rab-2 (Q65L) DA are able to induce locomotion defects when expressed in the neurons of wild type animals (Chun et al., 2008). Similar mutations in Rab7 have been shown to cause Charcot-Marie-Tooth Type 2B disease, an autosomal-dominant axonal neuropathy (Corbeel and Freson, 2008; Spang, 2004; Spinosa et al., 2008). This strongly suggests that indeed RAB-2 $n 777$ and $n 501$ mutant proteins are dominant active molecules, whose action ultimately produces a loss-of-function phenotype for several of the trafficking events that Rab2 is involved in. However, the dominant active rab-2 alleles might still supply enough RAB-2 function to initiate trafficking events required for endocytosis and engulfment.

\subsection{RAB-2 affects locomotion through the regulation of DCV signaling}

To study the role of RAB-2 in neuromuscular junction function, we took advantage of the dominant active unc-108/rab-2 alleles, which only affect locomotion. Dominant unc-108 mutants, $n 501$ and $n 777$, are aldicarb 
resistant, which suggests a decrease in acetylcholine secretion or muscle responsiveness. We excluded the possibility that postsynaptic side is affected by mutations in RAB-2 using an acetylcholine receptor agonist levamisole. Using HPF EM, we showed that the unc-108 mutants produce morphological defects at synapses or defects in the number and distribution of SVs or DCVs. Consistent with these observations, evoked synaptic and DCV exocytosis appeared normal in these unc-108 mutants. Furthermore, the SV recycling per se is not affected as judged by relatively normal distribution of GFP synaptobrevin. We showed that the dominant active RAB-2 mutations affect the maturation but not the release of DCVs. Since unc-108 mutants have impaired retention of soluble and transmembrane cargo during DCV maturation, a reasonable hypothesis is that neuropeptide function is impaired in unc-108 mutants and this causes the locomotory defect. However, this seems unlikely for several reasons. First, the DCV maturation defect in unc108 mutants does not affect the aggregated neuropeptide core of the vesicle. Consistent with this, a YFP-tagged neuropeptide in the double mutant that lacks EGL-3/PC2 processing enzyme does not lose YFP from the vesicle during maturation. Second, the levels of native, processed FMRFamide neuropeptides in the axons of unc-108 mutants are normal based on antibody staining. Furthermore, neuropeptide processing is not affected in dominant unc-108 mutants suggesting that functional and processing competent DCVs are generated. Again, FMRFamide neuropeptides, like all neuropeptides, are thought to be present in an aggregated (i.e. insoluble) state in DCVs. Third, knocking out peptide processing in unc-108 null mutants causes a strong synthetic phenotype, such that the locomotion rate and overall phenotype of 
the double mutant is not significantly different from an unc-31 null mutant (Edwards et al., in press). This again suggests that most, if not all, neuropeptide function remains intact in unc-108 null mutants.

The DCV maturation defect in unc-108 mutants may thus affect a function of DCVs unrelated to neuropeptides. One possibility is that RAB-2 mutations affect the sorting of GPCRs and/or their accessory proteins needed for GPCR function and specificity into DCVs (Bermak and Zhou, 2001). In support to this idea, stimulating the signaling cascade by either expressing the gain-offunction EGL-30 (Gaq subunit) or deleting DGK-1 (enzyme needed for attenuating the DAG signaling pathway) rescues the movement phenotype in unc-108 mutants. Also, cell culture expression of dominant active Rab2Q65L protein and siRNA mediated down regulation of Rab2 has been shown to both inhibit cell surface expression and signaling of $\alpha, \beta$-adrenergic and angiotensin II type 1 GPCRs, lending support to this idea (Dong and Wu, 2007). Indeed, the DCV maturation defect in unc-108 mutants is not restricted to soluble proteins; a DCV specific transmembrane protein, IDA-1/IA2 is affected to the same extent (Edwards et al., in press). Other possibilities for the missing function/ factor include soluble neurotrophins or growth factors secreted via the regulated secretory pathway (i.e. other regulated secretory proteins that do not coaggregate with neuropeptides in the core) (Brunner et al., 2007).

Interestingly, an aldicarb-resistance phenotype similar to that of unc-108 mutants is observed in mutants of protein kinase $\mathrm{C}, p k c-1$, in $C$. elegans (Sieburth et al., 2007). pkc-1 mutants have been shown to induce aldicarb 
resistance and movement defects by reducing the synaptic release of DCVs while the SV release is also unaffected. Therefore, in addition to neuropeptides yet unidentified GPCRs or growth factors could be cotransported in DCVs or released from DCVs, respectively. Due to the DCV maturation defects in unc-108 mutants one or more of these factors may be lost or excluded from unc-108 mutant DCVs. Thus we propose that the lack of DCV cargo due to mistrafficking during DCV maturation results in signaling defects at the neuromuscular junction leading to reduced locomotion. However, further experiments are needed to determine how UNC-108/RAB-2 affects movement defects and aldicarb sensitivity.

\subsection{Molecular function of RAB-2 during DCV maturation}

Rab2 has been shown to regulate vesicular transport and sorting at the Golgi apparatus (Chavrier et al., 1990; Tisdale, 1999; Tisdale and Balch, 1996; Tisdale and Jackson, 1998). Since we were not able to detect RAB-2 at synapses but found it abundantly and broadly localized to the Golgi and on vesicular structures surrounding the neuronal Golgi complexes, RAB-2 may affect trafficking events that arise from the Golgi or the closely associated endosomal system. Secretory granules and DCVs are generated at the late Golgi, most likely within the trans Golgi network (TGN) (Kim et al., 2006; Morvan and Tooze, 2008b; Tooze et al., 2001). Here, neuropeptide precursors, processing enzymes, and accessory proteins needed for DCV exocytosis have to be sorted into transport containers that subsequently bud off the Golgi apparatus. Our findings that neuropeptides in DCVs of dominant 
unc-108 mutant animals are fully processed, as shown by MALDI TOF MS analysis, and that the number of synaptic DCVs is not affected, rule out a requirement of RAB-2 in the initial generation of DCV precursors. The fact that DCV diameter is more variable and in some recessive alleles slightly enlarged suggest that mutations in RAB-2 might affect the fidelity of DCV biogenesis. In all unc-108/rab-2 alleles, however, we observed that the DCV cargo, NLP-21YFP colocalized more strongly with late endosomal and lysosomal markers as compared to wild type neurons, suggesting that the cargo is inappropriately lost from maturing DCVs. Moreover, the NLP-21 derived YFP levels are reduced in neuronal cell bodies of unc-108 mutants. Accordingly, the synaptic NLP-21 derived YFP fluorescence intensity could be rescued by blocking early endosomal function through expression of a dominant active GTP bound RAB-5 Q78L mutant. This strongly suggests that Rab2 is required to retain specific cargo during DCV maturation.

In addition to pro-neuropeptide and associated processing enzymes, constitutive secretory cargo, lysosomal enzymes, and other membrane proteins are also co-packaged into DCV precursors, forming iDCV budding off the Golgi. To become fully functional this inappropriate cargo has to be removed from iDCVs by clathrin mediated remodeling in order to allow maturation into mDCVs. This proofreading mechanism is important to ensure the generation of mDCVs that contain biologically active neuropeptides and undergo productive membrane fusion (Morvan and Tooze, 2008b; Tooze et al., 2001). In particular, after exit from the Golgi apparatus iDCVs have been reported to undergo syntaxin 6 dependent homotypic fusions in order to form 
a functionally distinct compartment from the Golgi (Morvan and Tooze, 2008b; Wendler et al., 2001). Later on, syntaxin 6 must be removed from iDCVs since it is no longer detectable on mDCVs. The cargo that is removed by clathrincoated vesicles is sorted to endosomes (Morvan and Tooze, 2008b). We propose that in unc-108 mutants this sorting step is affected such that cargo that would normally stay in maturing DCVs, such as soluble cargo like growth factors or yet unidentified GPCRs are lost to the endosomal system. This would also explain why in unc-108 the NLP-21 derived YFP cargo exhibits a stronger colocalization with a mCherry-syntaxin-6 (SYN-6) fusion, with which it would be removed from iDCVs.

Normally, mDCV cargo including neuropeptides that have been processed by their furin-like protein convertases are thought to be retained by aggregation and/or binding to a cargo receptor (Kim et al., 2006; Tooze et al., 2001). Acidification has also been shown to be important for retention of cargo and processed neuropeptides in maturing DCVs. Neutralization of acidic compartments indeed causes missorting of cargo and subsequent secretion from the cell (Moore et al., 1983; Wu et al., 2001). Interestingly, a recessive unc-108 mutation has been demonstrated to be defective in the acidification of the phagosomal lumen resulting in engulfment defects (Mangahas et al., 2008). Thus, RAB-2 function might be required to retain proton pumps required for acidification in maturing DCV. However, since proprotein convertases are only active under acidic $\mathrm{pH}$ conditions (Morvan and Tooze, 2008b), severe defects in acidification of iDCVs would also lead to neuropeptide processing defects, which are not observed in the dominant 
unc-108/rab-2 mutations or in the unc-108 null mutants (Edwards et al., in press). Therefore, we favor a model by which RAB-2 is required to stabilize a membrane domain required for retention of cargo in maturing DCVs.

Interestingly, Rab2, has recently been shown to bind to multiple Golgi localized coil-coil proteins (Golgins) and Golgi matrix proteins in a GTP dependent manner (Burguete et al., 2008; Short et al., 2001; Sinka et al., 2008). Golgins mark the identity of specific Golgi regions while Golgi matrix proteins are required to maintain the structural integrity of the Golgi (Panic et al., 2003). We showed that this interaction is preserved in C. elegans. The interactions of activated Rab2 with multiple Golgins might confer retention of Rab2 labeled vesicles and membrane compartments in close proximity to the Golgi stack. This local enrichment of Rab2 positive membrane compartments might be necessary for productive sorting and membrane fusion events during Rab2 dependent trafficking. This retention might also prevent Rab2 compartments to deviate to the cell periphery where the endosomal/lysosomal degradation route might be more dominant. Particularly, in light of the fact that newly formed iDCVs undergo homotypic membrane fusion and rearrangement events it might be beneficial and more efficient to keep these membrane structures in close proximity via dynamic interactions with Golgins at the Golgi (Tooze et al., 2001). Thus Rab2 could also help to orchestrate a dynamic network of functional interactions required for efficient maturation of DCVs. Since Rab2 is one of the most highly conserved Rab GTPases, such a role in DCV maturation is likely to be conserved throughout evolution. 


\subsection{RAB-2 might affect DCV maturation through APT-9/GGA dependent sorting between Golgi and endosomes}

It was shown previously that overexpression of dominant active Rab5 Q79L drives the fusion of the early endosomes and blocks the maturation of endosomal system (Simonsen et al., 1998). We showed that overexpression of dominant active RAB-5 Q78L rescues the NLP-21 derived YFP levels at unc-108 synapses. One possibility is that the blockage of early endosomal function prevents the delivery of soluble YFP cargo to the endosomal system. In agreement with this, the NLP-21 derived YFP levels in neuronal cell bodies are increased in unc-108 mutants upon expression of dominant active RAB-5. Also, blocking the early endosomal function by sequestering $\mathrm{PI}(3) \mathrm{P}$ through expression of 2xFYVE domain of EEA-1 has the same effect on NLP-21 derived YFP levels (Edwards et al., in press). Therefore, this suggests that interfering with early endosomal function either by driving homotypic fusion of endosomes or preventing Golgi derived transport carriers to tether to endosomes, leads to a suppression of the DCV sorting/maturation defects in unc-108/rab-2 mutants.

GGA proteins are monomeric clathrin adaptors and are responsible for appropriate sorting of the cargo into clathrin coated vesicles during DCV maturation (Kakhlon et al., 2006). In dominant unc-108 mutants, the only GGA protein homolog of C. elegans, APT-9 is less recruited to the early endosomes. One possibility is that mislocalization of APT-9 in unc-108 mutants promotes the access of inappropriate cargo into the clathrin coated 
vesicles during maturation, due to reduced fidelity of the mechanism. Due to this, the proteins that are destined to mDCVs would be lost in the endosomal/lysosomal system.

GGAs have been shown to interact with a Rabaptin-5-Rabex-5 complex, which is a Rab5 effectors (Zhai et al., 2003; Zhu et al., 2005). This interaction recruits GGAs to the early endosomes and blocks the clathrin coated vesicle formation (Mattera et al., 2003). Since GGAs are responsible for specific cargo recognition, it is possible that dominant active RAB-5 interferes with proper APT-9/GGA mediated clathrin coated formation at the maturing vesicles by promoting the interaction between Rabaptin-5-Rabex-5 complex and APT-9/GGA. Thus, dominant active RAB-5 blocks the loss of soluble cargo in unc-108 mutants. This would implement RAB-2 in APT-9/GGA dependent remodeling of iDCVs. However, how the mutations in RAB-2 affect APT/GGA function remains to be elucidated.

\subsection{RAB-2 cooperates with its effector RIC-19 during DCV maturation}

Once activated, Rab GTPases recruit effector molecules to target membranes. Recent work has shown that the diabetes autoantigen ICA69 is a direct Rab2 effector in human insulin producing $\beta$-cells. Rab2 recruits ICA69 to Golgi membranes in a GTP-dependent manner (Buffa et al., 2008). Interestingly, ICA69 is not only highly expressed in $\beta$-cells of the pancreas but 
also highly enriched in the brain (Pilon et al., 2000). The C. elegans ICA69 ortholog RIC-19 is specifically expressed in neurons, and ric-19 mutants have been reported to exhibit aldicarb-resistance (Pilon et al., 2000). Here we show that the aldicarb-resistance of ric-19 mutants is due to defects in the biogenesis of DCVs that are similar to the defects we observed in the dominant unc-108 mutants. We further demonstrate that RIC-19 is also a direct effector of RAB-2 in $C$. elegans. Our transgenic co-expression experiments are consistent with RIC-19 being recruited to Golgi membranes by active RAB-2.

Several lines of evidence suggest that RIC-19 and ICA69 function in a similar process during DCV biogenesis. Like ICA69, RIC-19 is found to colocalize with Golgi markers, but it is not found on vesicles at the synapse (Spitzenberger et al., 2003). Since RIC-19 is enriched at the Golgi in neuronal cell bodies in a RAB-2 dependent manner, it is likely that RIC-19 cooperates with RAB-2 during DCV maturation. Similarly, ICA69 shows a partial colocalization with immature secretory granules and dissociates from secretory granule membranes during further maturation (Spitzenberger et al., 2003). Furthermore, ICA69/RIC-19 contains a BAR domain which dimerizes and is known to recognize and bind to curved membranes found on vesicles and tubular carriers (Gallop and McMahon, 2005; Habermann, 2004; Zimmerberg and McLaughlin, 2004). Several BAR domain containing homoand heterodimeric proteins have also been shown to also interact with Rab GTPases (Habermann, 2004). Thus, the function of ICA69/RIC-19 together with RAB-2 might be to create and stabilize membrane domains on iDCVs 
during maturation. These membrane domains might be necessary to retain specific cargo in iDCVs and prevent their removal or inappropriate loss to the endosomal pathway through a clathrin-dependent mechanism. Although our data strongly suggest that RIC-19 and RAB-2 are acting together, we find different requirements for RAB-2 and RIC-19 during DCV maturation. Whereas in unc-108 mutants the size of DCVs is increased and more variable, in ric-19 mutants the size of synaptic DCVs is similar to wild type, but their number at release sites is slightly increased. This points towards a mechanism where, in the absence of RIC-19, components might be removed from maturing DCVs that are required at the synapse to regulate or facilitate the secretion. For example, RIC-19 might associate with iDCV membranes in the absence of RAB-2 via it's BAR domain in a curvature-dependent mechanism or through electrostatic interactions with the lipid bilayer. The interaction with RAB-2 might then be specifically required to stabilize these membrane domains to make them productive for sorting and retention of cargo.

\subsection{A RAB-5 dependent step required for DCV exocytosis?}

While the molecular mechanisms regulating SV release are somehow emerging, it is still unclear how DCV exocytosis is mediated and regulated (Holt et al., 2008; Sakaba et al., 2005; Suudhof, 2008; Xu and Xu, 2008). This is partly due to two facts: i) there is probably a heterogeneity in the regulation of dense core vesicle and secretory granule secretion in different cells and tissues; ii) furthermore, it is not clear so far what are the physiological stimuli 
and sites of release of DCVs in different neurons ( $X u$ and $X u, 2008)$. CAPS is the one of the best regulators known to be required for DCV exocytosis. (Ann et al., 1997; Berwin et al., 1998; Renden et al., 2001; Sieburth et al., 2007). In C. elegans, it has been shown that protein kinase $\mathrm{C} 1$ is also specifically required for DCV exocytosis as well (Sieburth et al., 2007).

We showed that dominant active RAB-5 Q78L rescues the loss of soluble cargo from maturing DCVs in unc-108 mutants probably by blocking the delivery of cargo from iDCV to endosomes. The mechanism of DCV maturation most likely involves clathrin dependent sorting step involving the monomeric clathrin adaptors, APT-9/GGA. In the mammalian system RNAi against GGA3 and overexpression of a dominant negative GGA3, missing the clathrin binding domain, blocks membrane remodelling of iDCVs. As a result, proteins that are responsible for homotypic iDCV fusion, synaptotagmin IV and syntaxin 6 remain on the mDCVs (Kakhlon et al., 2006). Thus, overexpressing dominant active RAB-5 might function similarly by retaining DCV factors on maturing DCVs which normally have to be removed for efficient DCV exocytosis. Indeed, the DCV secretion is blocked in dominant active RAB-5 expressing neurons. In agreement with this, the neuronal overexpression of RAB-5 causes movement defects and aldicarb resistance. A possible factor that could account for the blockage of DCV secretion might thus be synaptotagmin IV. It has been shown before that RNAi of synaptotagmin IV stimulates DCV release from posterior pituitary nerve terminal (Hu et al., 2008), while its overexpression blocks the secretion of secretory granules in PC12 cells (Ahras et al., 2009). Thus, synaptotagmin IV 
might be required in a first step for proper DCV maturation, but subsequently has to be removed to allow DCV exocytosis. Such a proofreading mechanism might guarantee that only mDCV that have successfully underwent maturation steps are competent for release. A RAB-5 dependent sorting step at the Golgi endosomal interface might therefore be required to remove synaptotagmin IV or similar factors the endosomal system.

The EM analysis of the synapse showed that distribution as well as the number of synaptic vesicles is not changed in dominant active RAB-5 expressing neurons. This is in agreement with previously published data (Shimizu et al., 2003; Wucherpfennig et al., 2003), suggesting that dominant active RAB-5 affects specifically DCVs, but not SVs. Interestingly, the stimulated secretion of SVs is not changed by expression of RAB-5 N142I. However, the steady state release is lowered (Shimizu et al., 2003).

Surprisingly, the NLP-21 derived YFP levels at the wild type synapses are not affected by dominant active RAB- 5 expression, even though the secretion is blocked. This means that the number of the DCVs and sorting of neuropeptides into the DCVs is not affected. One mechanism that controls the number of DCVs at the synapse is cleavage of the DCV transmembrane protein IA2/ICA512 (Trajkovski et al., 2008). Once a DCV has fused to the membrane, IA/ICA512 is cleaved and its cytoplasmic domain is liberated from the membrane. This C-terminal peptide is subsequently transported to the nucleus where it activates the production of new DCVs and their transport to the axon. Therefore, the blockage of secretion would not affect the number of 
the vesicles per se. This was confirmed by the EM analysis of dominant active RAB-5 expressing wild type synapses. Therefore, by blocking the DCV maturation, particularly removal of cargo from iDCVs to endosomes through expression of dominant active RAB-5, a yet unidentified factor may remain at the mDCVs and thus block the secretion.

In summary, we showed that DCV signaling is necessary for efficient neurotransmission at the neuromuscular junction. The maturation process is an important step for DCV to become fully functional. We showed that the Rab GTPases, RAB-2 and RAB-5, are both involved in DCV maturation. While RAB-2 is required to prevent loss of specific cargo from maturing DCVs, RAB5 is most likely involved in removal of specific cargo that is able to prevent DCV exocytosis. This study contributes to our understanding of involvement of Rab GTPases in neuronal trafficking. 


\section{References}

Ahmed, S., I.N. Maruyama, R. Kozma, J. Lee, S. Brenner, and L. Lim. 1992. The Caenorhabditis elegans unc-13 gene product is a phospholipiddependent high-affinity phorbol ester receptor. Biochem J. 287 ( Pt 3):995-9.

Alexandrov, K., H. Horiuchi, O. Steele-Mortimer, M.C. Seabra, and M. Zerial. 1994. Rab escort protein-1 is a multifunctional protein that accompanies newly prenylated rab proteins to their target membranes. EMBO J. 13:5262-73.

Anant, J.S., L. Desnoyers, M. Machius, B. Demeler, J.C. Hansen, K.D. Westover, J. Deisenhofer, and M.C. Seabra. 1998. Mechanism of Rab geranylgeranylation: formation of the catalytic ternary complex. Biochemistry. 37:12559-68.

Ann, K., J.A. Kowalchyk, K.M. Loyet, and T.F. Martin. 1997. Novel Ca2+binding protein (CAPS) related to UNC-31 required for Ca2+-activated exocytosis. J Biol Chem. 272:19637-40.

Aravamudan, B., T. Fergestad, W.S. Davis, C.K. Rodesch, and K. Broadie. 1999. Drosophila UNC-13 is essential for synaptic transmission. Nat Neurosci. 2:965-71.

Arhas, M., O. P. Grant, and S. Tooze. 2006. Synaptotagmin IV is necessary for the maturation of secretory granules in PC12 cells. J Cell Biol. 2:241-251.

Ashery, U., F. Varoqueaux, T. Voets, A. Betz, P. Thakur, H. Koch, E. Neher, N. Brose, and J. Rettig. 2000. Munc13-1 acts as a priming factor for large dense-core vesicles in bovine chromaffin cells. EMBO J. 19:358696.

Augustin, I., A. Betz, C. Herrmann, T. Jo, and N. Brose. 1999. Differential expression of two novel Munc13 proteins in rat brain. Biochem J. 337 ( Pt 3):363-71.

Avery, L., C.I. Bargmann, and H.R. Horvitz. 1993. The Caenorhabditis elegans unc-31 gene affects multiple nervous system-controlled functions. Genetics. 134:455-64.

Bermak, J.C., and Q.Y. Zhou. 2001. Accessory proteins in the biogenesis of G protein-coupled receptors. Mol Interv. 1:282-7.

Berwin, B., E. Floor, and T.F. Martin. 1998. CAPS (mammalian UNC-31) protein localizes to membranes involved in dense-core vesicle exocytosis. Neuron. 21:137-45.

Bock, J.B., H.T. Matern, A.A. Peden, and R.H. Scheller. 2001. A genomic perspective on membrane compartment organization. Nature. 409:83941.

Branicky, R., and S. Hekimi. 2006. What keeps C. elegans regular: the genetics of defecation. Trends Genet. 22:571-9.

Brenner, S. 1974. The genetics of Caenorhabditis elegans. Genetics. 77:7194. 
Brose, N., C. Rosenmund, and J. Rettig. 2000. Regulation of transmitter release by Unc-13 and its homologues. Curr Opin Neurobiol. 10:30311.

Brunner, Y., Y. Coute, M. lezzi, M. Foti, M. Fukuda, D.F. Hochstrasser, C.B. Wollheim, and J.C. Sanchez. 2007. Proteomics analysis of insulin secretory granules. Mol Cell Proteomics. 6:1007-17.

Bruns, D., and R. Jahn. 1995. Real-time measurement of transmitter release from single synaptic vesicles. Nature. 377:62-5.

Buffa, L., E. Fuchs, M. Pietropaolo, F. Barr, and M. Solimena. 2008. ICA69 is a novel Rab2 effector regulating ER-Golgi trafficking in insulinoma cells. Eur J Cell Biol. 87:197-209.

Burguete, A.S., T.D. Fenn, A.T. Brunger, and S.R. Pfeffer. 2008. Rab and Arl GTPase family members cooperate in the localization of the golgin GCC185. Cell. 132:286-98.

Chavrier, P., R.G. Parton, H.P. Hauri, K. Simons, and M. Zerial. 1990. Localization of low molecular weight GTP binding proteins to exocytic and endocytic compartments. Cell. 62:317-29.

Chun, D.K., J.M. McEwen, M. Burbea, and J.M. Kaplan. 2008. UNC-108/Rab2 regulates postendocytic trafficking in Caenorhabditis elegans. Mol Biol Cell. 19:2682-95.

Corbeel, L., and K. Freson. 2008. Rab proteins and Rab-associated proteins: major actors in the mechanism of protein-trafficking disorders. Eur $J$ Pediatr. 167:723-9.

Deinhardt, K., S. Salinas, C. Verastegui, R. Watson, D. Worth, S. Hanrahan, C. Bucci, and G. Schiavo. 2006. Rab5 and Rab7 control endocytic sorting along the axonal retrograde transport pathway. Neuron. 52:293305.

Deneka, M., M. Neeft, and P. van der Sluijs. 2003. Regulation of membrane transport by rab GTPases. Crit Rev Biochem Mol Biol. 38:121-42.

Dennes, A., C. Cromme, K. Suresh, N.S. Kumar, J.A. Eble, A. Hahnenkamp, and R. Pohlmann. 2005. The novel Drosophila lysosomal enzyme receptor protein mediates lysosomal sorting in mammalian cells and binds mammalian and Drosophila GGA adaptors. J Biol Chem. 280:12849-57.

Di Giovanni, S., C.D. Knights, M. Rao, A. Yakovlev, J. Beers, J. Catania, M.L. Avantaggiati, and A.I. Faden. 2006. The tumor suppressor protein p53 is required for neurite outgrowth and axon regeneration. EMBO J. 25:4084-96.

Dong, C., and G. Wu. 2007. Regulation of anterograde transport of adrenergic and angiotensin II receptors by Rab2 and Rab6 GTPases. Cell Signal. 19:2388-99.

Dulubova, I., X. Lou, J. Lu, I. Huryeva, A. Alam, R. Schneggenburger, T.C. Sudhof, and J. Rizo. 2005. A Munc13/RIM/Rab3 tripartite complex: from priming to plasticity? EMBO J. 24:2839-50.

Dumas, J.J., Z. Zhu, J.L. Connolly, and D.G. Lambright. 1999. Structural basis of activation and GTP hydrolysis in Rab proteins. Structure. 7:413-23.

Edwards, S. L., N.K. Charlie, J.E. Richmond, J. Hegermann, S. Eimer and K.G. Miller. 2009. Impaired dense core vesicle maturation in C. elegans mutants lacking RAB-2. J Cell Biol. 186: in press 
Fukuda, M. 2008. Regulation of secretory vesicle traffic by Rab small GTPases. Cell Mol Life Sci. 65:2801-13.

Gallop, J.L., and H.T. McMahon. 2005. BAR domains and membrane curvature: bringing your curves to the BAR. Biochem Soc Symp:22331.

Gally, C., S. Eimer, J.E. Richmond, and J.L. Bessereau. 2004. A transmembrane protein required for acetylcholine receptor clustering in Caenorhabditis elegans. Nature. 431:578-82.

Geppert, M., V.Y. Bolshakov, S.A. Siegelbaum, K. Takei, P. De Camilli, R.E. Hammer, and T.C. Sudhof. 1994. The role of Rab3A in neurotransmitter release. Nature. 369:493-7.

Gillis, K.D., R. Mossner, and E. Neher. 1996. Protein kinase C enhances exocytosis from chromaffin cells by increasing the size of the readily releasable pool of secretory granules. Neuron. 16:1209-20.

Gracheva, E.O., A.O. Burdina, D. Touroutine, M. Berthelot-Grosjean, H. Parekh, and J.E. Richmond. 2007a. Tomosyn negatively regulates both synaptic transmitter and neuropeptide release at the $C$. elegans neuromuscular junction. J Physiol. 585:705-9.

Gracheva, E.O., A.O. Burdina, D. Touroutine, M. Berthelot-Grosjean, H. Parekh, and J.E. Richmond. 2007b. Tomosyn negatively regulates CAPS-dependent peptide release at Caenorhabditis elegans synapses. J Neurosci. 27:10176-84.

Gracheva, E.O., G. Hadwiger, M.L. Nonet, and J.E. Richmond. 2008. Direct interactions between C. elegans RAB-3 and Rim provide a mechanism to target vesicles to the presynaptic density. Neurosci Lett. 444:137-42.

Habermann, B. 2004. The BAR-domain family of proteins: a case of bending and binding? EMBO Rep. 5:250-5.

Holt, M., D. Riedel, A. Stein, C. Schuette, and R. Jahn. 2008. Synaptic vesicles are constitutively active fusion machines that function independently of Ca2+. Curr Biol. 18:715-22.

Hu, Z.T., M.R. Chen, Z. Ping, Y.M. Dong, R.Y. Zhang, T. Xu, and Z.X. Wu. 2008. Synaptotagmin IV regulates dense core vesicle (DCV) release in LbetaT2 cells. Biochem Biophys Res Commun. 371:781-6.

Hume, A.N., L.M. Collinson, A. Rapak, A.Q. Gomes, C.R. Hopkins, and M.C. Seabra. 2001. Rab27a regulates the peripheral distribution of melanosomes in melanocytes. Journal of Cell Biology. 152:795-808.

Husson, S.J., E. Clynen, G. Baggerman, A. De Loof, and L. Schoofs. 2005. Discovering neuropeptides in Caenorhabditis elegans by two dimensional liquid chromatography and mass spectrometry. Biochem Biophys Res Commun. 335:76-86.

Husson, S.J., E. Clynen, G. Baggerman, T. Janssen, and L. Schoofs. 2006. Defective processing of neuropeptide precursors in Caenorhabditis elegans lacking proprotein convertase 2 (KPC-2/EGL-3): mutant analysis by mass spectrometry. J Neurochem. 98:1999-2012.

Husson, S.J., T. Janssen, G. Baggerman, B. Bogert, A.H. Kahn-Kirby, K. Ashrafi, and L. Schoofs. 2007a. Impaired processing of FLP and NLP peptides in carboxypeptidase E (EGL-21)-deficient Caenorhabditis elegans as analyzed by mass spectrometry. J Neurochem. 102:246-60. 
Husson, S.J., I. Mertens, T. Janssen, M. Lindemans, and L. Schoofs. 2007b. Neuropeptidergic signaling in the nematode Caenorhabditis elegans. Prog Neurobiol. 82:33-55.

Husson, S.J., and L. Schoofs. 2007. Processing of neuropeptide precursors in Caenorhabditis elegans. Commun Agric Appl Biol Sci. 72:199-203.

Jacob, T.C., and J.M. Kaplan. 2003. The EGL-21 carboxypeptidase E facilitates acetylcholine release at Caenorhabditis elegans neuromuscular junctions. J Neurosci. 23:2122-30.

Jorgensen, E.M. 2005. Gaba. WormBook:1-13.

Kakhlon, O., P. Sakya, B. Larijani, R. Watson, and S.A. Tooze. 2006. GGA function is required for maturation of neuroendocrine secretory granules. EMBO J. 25:1590-602.

Kamath, R.S., M. Martinez-Campos, P. Zipperlen, A.G. Fraser, and J. Ahringer. 2001. Effectiveness of specific RNA-mediated interference through ingested double-stranded RNA in Caenorhabditis elegans. Genome Biol. 2:RESEARCH0002.

Kass, J., T.C. Jacob, P. Kim, and J.M. Kaplan. 2001. The EGL-3 proprotein convertase regulates mechanosensory responses of Caenorhabditis elegans. J Neurosci. 21:9265-72.

Kim, T., M.C. Gondre-Lewis, I. Arnaoutova, and Y.P. Loh. 2006. Dense-core secretory granule biogenesis. Physiology (Bethesda). 21:124-33.

Kiyonaka, S., M. Wakamori, T. Miki, Y. Uriu, M. Nonaka, H. Bito, A.M. Beedle, E. Mori, Y. Hara, M. De Waard, M. Kanagawa, M. Itakura, M. Takahashi, K.P. Campbell, and Y. Mori. 2007. RIM1 confers sustained activity and neurotransmitter vesicle anchoring to presynaptic Ca2+ channels. Nat Neurosci. 10:691-701.

Koh, T.W., and H.J. Bellen. 2003. Synaptotagmin I, a Ca2+ sensor for neurotransmitter release. Trends Neurosci. 26:413-22.

Kostich, M., A. Fire, and D.M. Fambrough. 2000. Identification and moleculargenetic characterization of a LAMP/CD68-like protein from Caenorhabditis elegans. J Cell Sci. 113 ( Pt 14):2595-606.

Lackner, M.R., S.J. Nurrish, and J.M. Kaplan. 1999. Facilitation of synaptic transmission by EGL-30 Gqalpha and EGL-8 PLCbeta: DAG binding to UNC-13 is required to stimulate acetylcholine release. Neuron. 24:33546.

Lewis, J.A., C.H. Wu, J.H. Levine, and H. Berg. 1980. Levamisole-resistant mutants of the nematode Caenorhabditis elegans appear to lack pharmacological acetylcholine receptors. Neuroscience. 5:967-89.

Li, C., K. Kim, and L.S. Nelson. 1999. FMRFamide-related neuropeptide gene family in Caenorhabditis elegans. Brain Res. 848:26-34.

Li, C., K. Takei, M. Geppert, L. Daniell, K. Stenius, E.R. Chapman, R. Jahn, P. De Camilli, and T.C. Sudhof. 1994. Synaptic targeting of rabphilin-3A, a synaptic vesicle $\mathrm{Ca} 2+/$ phospholipid-binding protein, depends on rab3A/3C. Neuron. 13:885-98.

Lonart, G., and T.C. Sudhof. 2000. Assembly of SNARE core complexes prior to neurotransmitter release sets the readily releasable pool of synaptic vesicles. J Biol Chem. 275:27703-7.

Lu, Q., Y. Zhang, T. Hu, P. Guo, W. Li, and X. Wang. 2008. C. elegans Rab GTPase 2 is required for the degradation of apoptotic cells. Development. 135:1069-80. 
Mahoney, T.R., Q. Liu, T. Itoh, S. Luo, G. Hadwiger, R. Vincent, Z.W. Wang, M. Fukuda, and M.L. Nonet. 2006. Regulation of synaptic transmission by RAB-3 and RAB-27 in Caenorhabditis elegans. Mol Biol Cell. 17:2617-25.

Mangahas, P.M., X. Yu, K.G. Miller, and Z. Zhou. 2008. The small GTPase Rab2 functions in the removal of apoptotic cells in Caenorhabditis elegans. J Cell Biol. 180:357-73.

Maruyama, I.N., and S. Brenner. 1991. A phorbol ester/diacylglycerol-binding protein encoded by the unc-13 gene of Caenorhabditis elegans. Proc Natl Acad Sci U S A. 88:5729-33.

Marza, E., T. Long, A. Saiardi, M. Sumakovic, S. Eimer, D.H. Hall, and G.M. Lesa. 2008. Polyunsaturated fatty acids influence synaptojanin localization to regulate synaptic vesicle recycling. Mol Biol Cell. 19:83342.

Matsui, Y., A. Kikuchi, S. Araki, Y. Hata, J. Kondo, Y. Teranishi, and Y. Takai. 1990. Molecular cloning and characterization of a novel type of regulatory protein (GDI) for smg p25A, a ras p21-like GTP-binding protein. Mol Cell Biol. 10:4116-22.

Mattera, R., C.N. Arighi, R. Lodge, M. Zerial, and J.S. Bonifacino. 2003. Divalent interaction of the GGAs with the Rabaptin-5-Rabex-5 complex. EMBO J. 22:78-88.

Mello, C.C., J.M. Kramer, D. Stinchcomb, and V. Ambros. 1991. Efficient gene transfer in C.elegans: extrachromosomal maintenance and integration of transforming sequences. EMBO J. 10:3959-70.

Miller, K.G., M.D. Emerson, and J.B. Rand. 1999. Goalpha and diacylglycerol kinase negatively regulate the Gqalpha pathway in C. elegans. Neuron. 24:323-33.

Milosevic, I., J.B. Sorensen, T. Lang, M. Krauss, G. Nagy, V. Haucke, R. Jahn, and E. Neher. 2005. Plasmalemmal phosphatidylinositol-4,5bisphosphate level regulates the releasable vesicle pool size in chromaffin cells. J Neurosci. 25:2557-65.

Misonou, H., M. Ohara-Imaizumi, T. Murakami, M. Kawasaki, K. Ikeda, T. Wakai, and K. Kumakura. 1998. Protein kinase C controls the priming step of regulated exocytosis in adrenal chromaffin cells. Cell $\mathrm{Mol}$ Neurobiol. 18:379-90.

Moore, H.P., B. Gumbiner, and R.B. Kelly. 1983. Chloroquine diverts ACTH from a regulated to a constitutive secretory pathway in AtT-20 cells. Nature. 302:434-6.

Morvan, J., and S.A. Tooze. 2008a. Discovery and progress in our understanding of the regulated secretory pathway in neuroendocrine cells. Histochem Cell Biol. 129:243-52.

Morvan, J., and S.A. Tooze. 2008b. Discovery and progress in our understanding of the regulated secretory pathway in neuroendocrine cells. Histochem Cell Biol. 129:243-252.

Ng, E.L., and B.L. Tang. 2008. Rab GTPases and their roles in brain neurons and glia. Brain Res Rev. 58:236-46.

Nonet, M.L., J.E. Staunton, M.P. Kilgard, T. Fergestad, E. Hartwieg, H.R. Horvitz, E.M. Jorgensen, and B.J. Meyer. 1997. Caenorhabditis elegans rab-3 mutant synapses exhibit impaired function and are partially depleted of vesicles. J Neurosci. 17:8061-73. 
Nurrish, S., L. Segalat, and J.M. Kaplan. 1999. Serotonin inhibition of synaptic transmission: Galpha(0) decreases the abundance of UNC-13 at release sites. Neuron. 24:231-42.

Ostermeier, C., and A.T. Brunger. 1999. Structural basis of Rab effector specificity: crystal structure of the small $G$ protein Rab3A complexed with the effector domain of rabphilin-3A. Cell. 96:363-74.

Paduch, M., F. Jelen, and J. Otlewski. 2001. Structure of small G proteins and their regulators. Acta Biochim Pol. 48:829-50.

Pan, J.Y., and M. Wessling-Resnick. 1998. GEF-mediated GDP/GTP exchange by monomeric GTPases: a regulatory role for $\mathrm{Mg} 2+$ ? Bioessays. 20:516-21.

Panic, B., O. Perisic, D.B. Veprintsev, R.L. Williams, and S. Munro. 2003. Structural basis for Arl1-dependent targeting of homodimeric GRIP domains to the Golgi apparatus. Mol Cell. 12:863-74.

Park, E.C., and H.R. Horvitz. 1986. Mutations with dominant effects on the behavior and morphology of the nematode Caenorhabditis elegans. Genetics. 113:821-52.

Patel, M.R., E.K. Lehrman, V.Y. Poon, J.G. Crump, M. Zhen, C.I. Bargmann, and K. Shen. 2006. Hierarchical assembly of presynaptic components in defined C. elegans synapses. Nat Neurosci. 9:1488-98.

Pereira-Leal, J.B., A.N. Hume, and M.C. Seabra. 2001. Prenylation of Rab GTPases: molecular mechanisms and involvement in genetic disease. Febs Letters. 498:197-200.

Pereira-Leal, J.B., and M.C. Seabra. 2001. Evolution of the Rab family of small GTP-binding proteins. J Mol Biol. 313:889-901.

Perrais, D., I.C. Kleppe, J.W. Taraska, and W. Almers. 2004. Recapture after exocytosis causes differential retention of protein in granules of bovine chromaffin cells. J Physiol. 560:413-28.

Pfeffer, S., and D. Aivazian. 2004. Targeting Rab GTPases to distinct membrane compartments. Nat Rev Mol Cell Biol. 5:886-96.

Pilon, M., X.R. Peng, A.M. Spence, R.H. Plasterk, and H.M. Dosch. 2000. The diabetes autoantigen ICA69 and its Caenorhabditis elegans homologue, ric-19, are conserved regulators of neuroendocrine secretion. Mol Biol Cell. 11:3277-88.

Renden, R., B. Berwin, W. Davis, K. Ann, C.T. Chin, R. Kreber, B. Ganetzky, T.F. Martin, and K. Broadie. 2001. Drosophila CAPS is an essential gene that regulates dense-core vesicle release and synaptic vesicle fusion. Neuron. 31:421-37.

Reynolds, E.S. 1963. The use of lead citrate at high $\mathrm{pH}$ as an electron-opaque stain in electron microscopy. J Cell Biol. 17:208-12.

Richmond, J.E., and K.S. Broadie. 2002. The synaptic vesicle cycle: exocytosis and endocytosis in Drosophila and C. elegans. Curr Opin Neurobiol. 12:499-507.

Richmond, J.E., W.S. Davis, and E.M. Jorgensen. 1999. UNC-13 is required for synaptic vesicle fusion in C. elegans. Nat Neurosci. 2:959-64.

Richmond, J.E., R.M. Weimer, and E.M. Jorgensen. 2001. An open form of syntaxin bypasses the requirement for UNC-13 in vesicle priming. Nature. 412:338-41.

Rostaing, P., E. Real, L. Siksou, J.P. Lechaire, T. Boudier, T.M. Boeckers, F. Gertler, E.D. Gundelfinger, A. Triller, and S. Marty. 2006. Analysis of 
synaptic ultrastructure without fixative using high-pressure freezing and tomography. Eur J Neurosci. 24:3463-74.

Sakaba, T., A. Stein, R. Jahn, and E. Neher. 2005. Distinct kinetic changes in neurotransmitter release after SNARE protein cleavage. Science. 309:491-4.

Sakane, A., S. Manabe, H. Ishizaki, M. Tanaka-Okamoto, E. Kiyokage, K. Toida, T. Yoshida, J. Miyoshi, H. Kamiya, Y. Takai, and T. Sasaki. 2006. Rab3 GTPase-activating protein regulates synaptic transmission and plasticity through the inactivation of Rab3. Proc Natl Acad Sci U S A. 103:10029-34.

Sassa, T., H. Ogawa, M. Kimoto, and R. Hosono. 1996. The synaptic protein UNC-18 is phosphorylated by protein kinase C. Neurochem Int. 29:54352.

Saxena, S., M. Singh, K. Engisch, M. Fukuda, and S. Kaur. 2005. Rab proteins regulate epithelial sodium channel activity in colonic epithelial HT-29 cells. Biochem Biophys Res Commun. 337:1219-23.

Schafer, W.R. 2006. Neurophysiological methods in C. elegans: an introduction. WormBook:1-4.

Schlichting, I., S.C. Almo, G. Rapp, K. Wilson, K. Petratos, A. Lentfer, A. Wittinghofer, W. Kabsch, E.F. Pai, G.A. Petsko, and et al. 1990. Timeresolved X-ray crystallographic study of the conformational change in Ha-Ras p21 protein on GTP hydrolysis. Nature. 345:309-15.

Schluter, O.M., F. Schmitz, R. Jahn, C. Rosenmund, and T.C. Sudhof. 2004. A complete genetic analysis of neuronal Rab3 function. $J$ Neurosci. 24:6629-37.

Schoch, S., P.E. Castillo, T. Jo, K. Mukherjee, M. Geppert, Y. Wang, F. Schmitz, R.C. Malenka, and T.C. Sudhof. 2002. RIM1alpha forms a protein scaffold for regulating neurotransmitter release at the active zone. Nature. 415:321-6.

Scott, G.K., H. Fei, L. Thomas, G.R. Medigeshi, and G. Thomas. 2006. A PACS-1, GGA3 and CK2 complex regulates Cl-MPR trafficking. EMBO J. 25:4423-35.

Scott, P.M., P.S. Bilodeau, O. Zhdankina, S.C. Winistorfer, M.J. Hauglund, M.M. Allaman, W.R. Kearney, A.D. Robertson, A.L. Boman, and R.C. Piper. 2004. GGA proteins bind ubiquitin to facilitate sorting at the trans-Golgi network. Nat Cell Biol. 6:252-9.

Seabra, M.C. 1996. Nucleotide dependence of Rab geranylgeranylation. Rab escort protein interacts preferentially with GDP-bound Rab. J Biol Chem. 271:14398-404.

Searl, T.J., and E.M. Silinsky. 2003. Phorbol esters and adenosine affect the readily releasable neurotransmitter pool by different mechanisms at amphibian motor nerve endings. J Physiol. 553:445-56.

Shapiro, A.D., M.A. Riederer, and S.R. Pfeffer. 1993. Biochemical analysis of rab9, a ras-like GTPase involved in protein transport from late endosomes to the trans Golgi network. J Biol Chem. 268:6925-31.

Sheffield, P., S. Garrard, and Z. Derewenda. 1999. Overcoming expression and purification problems of RhoGDI using a family of "parallel" expression vectors. Protein Expr Purif. 15:34-9. 
Shen, F., and M.C. Seabra. 1996. Mechanism of digeranylgeranylation of Rab proteins. Formation of a complex between monogeranylgeranyl-Rab and Rab escort protein. J Biol Chem. 271:3692-8.

Shimizu, H., S. Kawamura, and K. Ozaki. 2003. An essential role of Rab5 in uniformity of synaptic vesicle size. J Cell Sci. 116:3583-90.

Short, B., C. Preisinger, R. Korner, R. Kopajtich, O. Byron, and F.A. Barr. 2001. A GRASP55-rab2 effector complex linking Golgi structure to membrane traffic. J Cell Biol. 155:877-83.

Sieburth, D., Q. Ch'ng, M. Dybbs, M. Tavazoie, S. Kennedy, D. Wang, D. Dupuy, J.F. Rual, D.E. Hill, M. Vidal, G. Ruvkun, and J.M. Kaplan. 2005. Systematic analysis of genes required for synapse structure and function. Nature. 436:510-7.

Sieburth, D., J.M. Madison, and J.M. Kaplan. 2007. PKC-1 regulates secretion of neuropeptides. Nat Neurosci. 10:49-57.

Silinsky, E.M., and T.J. Searl. 2003. Phorbol esters and neurotransmitter release: more than just protein kinase C? Br J Pharmacol. 138:1191201.

Simmer, F., C. Moorman, A.M. Van Der Linden, E. Kuijk, P.V. Van Den Berghe, R. Kamath, A.G. Fraser, J. Ahringer, and R.H. Plasterk. 2003. Genome-Wide RNAi of C. elegans Using the Hypersensitive rrf-3 Strain Reveals Novel Gene Functions. PLoS Biol. 1:E12.

Simonsen, A., R. Lippe, S. Christoforidis, J.M. Gaullier, A. Brech, J. Callaghan, B.H. Toh, C. Murphy, M. Zerial, and H. Stenmark. 1998. EEA1 links $\mathrm{PI}(3) \mathrm{K}$ function to Rab5 regulation of endosome fusion. Nature. 394:494-8.

Sinka, R., A.K. Gillingham, V. Kondylis, and S. Munro. 2008. Golgi coiled-coil proteins contain multiple binding sites for Rab family $G$ proteins. J Cell Biol. 183:607-15.

Sivars, U., D. Aivazian, and S.R. Pfeffer. 2003. Yip3 catalyses the dissociation of endosomal Rab-GDI complexes. Nature. 425:856-9.

Soldati, T., A.D. Shapiro, A.B. Svejstrup, and S.R. Pfeffer. 1994. Membrane targeting of the small GTPase Rab9 is accompanied by nucleotide exchange. Nature. 369:76-8.

Spang, A. 2004. Vesicle transport: a close collaboration of Rabs and effectors. Curr Biol. 14:R33-4.

Spinosa, M.R., C. Progida, A. De Luca, A.M. Colucci, P. Alifano, and C. Bucci. 2008. Functional characterization of Rab7 mutant proteins associated with Charcot-Marie-Tooth type 2B disease. J Neurosci. 28:1640-8.

Spitzenberger, F., S. Pietropaolo, P. Verkade, B. Habermann, S. LacasGervais, H. Mziaut, M. Pietropaolo, and M. Solimena. 2003. Islet cell autoantigen of $69 \mathrm{kDa}$ is an arfaptin-related protein associated with the Golgi complex of insulinoma INS-1 cells. J Biol Chem. 278:26166-73.

Stauffer, T.P., S. Ahn, and T. Meyer. 1998. Receptor-induced transient reduction in plasma membrane Ptdlns $(4,5) \mathrm{P} 2$ concentration monitored in living cells. Curr Biol. 8:343-6.

Stenmark, H., R.G. Parton, O. Steele-Mortimer, A. Lutcke, J. Gruenberg, and M. Zerial. 1994a. Inhibition of rab5 GTPase activity stimulates membrane fusion in endocytosis. EMBO J. 13:1287-96. 
Stenmark, H., A. Valencia, O. Martinez, O. Ullrich, B. Goud, and M. Zerial. 1994b. Distinct Structural Elements of Rab5 Define Its Functional Specificity. Embo Journal. 13:575-583.

Stevens, C.F., and J.M. Sullivan. 1998. Regulation of the readily releasable vesicle pool by protein kinase C. Neuron. 21:885-93.

Stevens, D.R., Z.X. Wu, U. Matti, H.J. Junge, C. Schirra, U. Becherer, S.M. Wojcik, N. Brose, and J. Rettig. 2005. Identification of the minimal protein domain required for priming activity of Munc13-1. Curr Biol. 15:2243-8.

Stinchcombe, J.C., D.C. Barral, E.H. Mules, S. Booth, A.N. Hume, L.M. Machesky, M.C. Seabra, and G.M. Griffiths. 2001. Rab27a is required for regulated secretion in cytotoxic T lymphocytes. Journal of Cell Biology. 152:825-833.

Stroupe, M.E., C. Xu, B.L. Goode, and N. Grigorieff. 2009. Actin filament labels for localizing protein components in large complexes viewed by electron microscopy. RNA. 15:244-8.

Suudhof, T.C. 2008. Neurotransmitter release. Handb Exp Pharmacol:1-21.

Takamori, S., M. Holt, K. Stenius, E.A. Lemke, M. Gronborg, D. Riedel, H. Urlaub, S. Schenck, B. Brugger, P. Ringler, S.A. Muller, B. Rammner, F. Grater, J.S. Hub, B.L. De Groot, G. Mieskes, Y. Moriyama, J. Klingauf, H. Grubmuller, J. Heuser, F. Wieland, and R. Jahn. 2006. Molecular anatomy of a trafficking organelle. Cell. 127:831-46.

Thomas, J.H. 1990. Genetic analysis of defecation in Caenorhabditis elegans. Genetics. 124:855-72.

Tisdale, E.J. 1999. A Rab2 mutant with impaired GTPase activity stimulates vesicle formation from pre-Golgi intermediates. Mol Biol Cell. 10:183749.

Tisdale, E.J., and W.E. Balch. 1996. Rab2 is essential for the maturation of pre-Golgi intermediates. J Biol Chem. 271:29372-9.

Tisdale, E.J., and M.R. Jackson. 1998. Rab2 protein enhances coatomer recruitment to pre-Golgi intermediates. J Biol Chem. 273:17269-77.

Tooze, S.A., G.J. Martens, and W.B. Huttner. 2001. Secretory granule biogenesis: rafting to the SNARE. Trends Cell Biol. 11:116-22.

Trajkovski, M., H. Mziaut, S. Schubert, Y. Kalaidzidis, A. Altkruger, and M. Solimena. 2008. Regulation of insulin granule turnover in pancreatic beta-cells by cleaved ICA512. J Biol Chem. 283:33719-29.

Ullrich, O., H. Horiuchi, C. Bucci, and M. Zerial. 1994. Membrane association of Rab5 mediated by GDP-dissociation inhibitor and accompanied by GDP/GTP exchange. Nature. 368:157-60.

Ullrich, O., H. Stenmark, K. Alexandrov, L.A. Huber, K. Kaibuchi, T. Sasaki, Y. Takai, and M. Zerial. 1993. Rab GDP dissociation inhibitor as a general regulator for the membrane association of rab proteins. $J$ Biol Chem. 268:18143-50.

Valencia, A., M. Kjeldgaard, E.F. Pai, and C. Sander. 1991. GTPase domains of ras p21 oncogene protein and elongation factor Tu: analysis of three-dimensional structures, sequence families, and functional sites. Proc Natl Acad Sci U S A. 88:5443-7.

Varnai, P., and T. Balla. 1998. Visualization of phosphoinositides that bind pleckstrin homology domains: calcium- and agonist-induced dynamic 
changes and relationship to myo-[3H]inositol-labeled phosphoinositide pools. J Cell Biol. 143:501-10.

Vonmollard, G.F., G.A. Mignery, M. Baumert, M.S. Perin, T.J. Hanson, P.M. Burger, R. Jahn, and T.C. Sudhof. 1990. Rab3 Is a Small Gtp-Binding Protein Exclusively Localized to Synaptic Vesicles. Proceedings of the National Academy of Sciences of the United States of America. 87:1988-1992.

Walrond, J.P., and A.O. Stretton. 1985a. Excitatory and inhibitory activity in the dorsal musculature of the nematode Ascaris evoked by single dorsal excitatory motonerons. J Neurosci. 5:16-22.

Walrond, J.P., and A.O. Stretton. 1985b. Reciprocal inhibition in the motor nervous system of the nematode Ascaris: direct control of ventral inhibitory motoneurons by dorsal excitatory motoneurons. J Neurosci. 5:9-15.

Waters, J., and S.J. Smith. 2000. Phorbol esters potentiate evoked and spontaneous release by different presynaptic mechanisms. J Neurosci. 20:7863-70.

Weimer, R.M., E.O. Gracheva, O. Meyrignac, K.G. Miller, J.E. Richmond, and J.L. Bessereau. 2006. UNC-13 and UNC-10/rim localize synaptic vesicles to specific membrane domains. J Neurosci. 26:8040-7.

Wendler, F., L. Page, S. Urbe, and S.A. Tooze. 2001. Homotypic fusion of immature secretory granules during maturation requires syntaxin 6 . Mol Biol Cell. 12:1699-709.

White, S.L., and P.S. Rainbow. 1986. A preliminary study of Cu-, Cd- and Znbinding components in the hepatopancreas of Palaemon elegans (Crustacea: Decapoda). Comp Biochem Physiol C. 83:111-6.

Wolinsky, E., and J. Way. 1990. The behavioral genetics of Caenorhabditis elegans. Behav Genet. 20:169-89.

Wu, M.M., M. Grabe, S. Adams, R.Y. Tsien, H.P. Moore, and T.E. Machen. 2001. Mechanisms of $\mathrm{pH}$ regulation in the regulated secretory pathway. J Biol Chem. 276:33027-35.

Wucherpfennig, T., M. Wilsch-Brauninger, and M. Gonzalez-Gaitan. 2003. Role of Drosophila Rab5 during endosomal trafficking at the synapse and evoked neurotransmitter release. J Cell Biol. 161:609-24.

$\mathrm{Xu}$, T., and P. Xu. 2008. Searching for molecular players differentially involved in neurotransmitter and neuropeptide release. Neurochem Res. 33:1915-9.

Zacchi, P., H. Stenmark, R.G. Parton, D. Orioli, F. Lim, A. Giner, I. Mellman, M. Zerial, and C. Murphy. 1998. Rab17 regulates membrane trafficking through apical recycling endosomes in polarized epithelial cells. Journal of Cell Biology. 140:1039-1053.

Zerial, M., and H. McBride. 2001. Rab proteins as membrane organizers. Nat Rev Mol Cell Biol. 2:107-17.

Zhai, P., X. He, J. Liu, N. Wakeham, G. Zhu, G. Li, J. Tang, and X.C. Zhang. 2003. The interaction of the human GGA1 GAT domain with rabaptin-5 is mediated by residues on its three-helix bundle. Biochemistry. 42:13901-8.

Zhang, J., K.L. Schulze, P.R. Hiesinger, K. Suyama, S. Wang, M. Fish, M. Acar, R.A. Hoskins, H.J. Bellen, and M.P. Scott. 2007. Thirty-one flavors of Drosophila rab proteins. Genetics. 176:1307-22. 
Zhou, K.M., Y.M. Dong, Q. Ge, D. Zhu, W. Zhou, X.G. Lin, T. Liang, Z.X. Wu, and T. Xu. 2007. PKA activation bypasses the requirement for UNC-31 in the docking of dense core vesicles from $\mathrm{C}$. elegans neurons. Neuron. 56:657-69.

Zhu, G., P. Zhai, N. Wakeham, X. He, and X.C. Zhang. 2005. Analysis of the interaction between GGA1 GAT domain and Rabaptin-5. Methods Enzymol. 403:583-92.

Zimmerberg, J., and S. McLaughlin. 2004. Membrane curvature: how BAR domains bend bilayers. Curr Biol. 14:R250-2. 


\section{Curriculum vitae}

\section{PERSONAL DATA}

Name: $\quad$ Marija Sumakovic

Date and place of birth : April $2^{\text {nd }}, 1981$. Smederevo

Address : $\quad$ Buergerstrasse 11a, Goettingen

Country : $\quad$ Germany

E-mail: $\quad$ msumako@gwdg.de

\section{EDUCATION}

2005- International Max Planck Research School for Neurosciences, Goettingen, Germany

1999-2005. Faculty of Biology, University of Belgrade

Department of Molecular Biology and Physiology

-Genetic Engineering and Biotechnology-

\section{AWARDS AND SCHOLARSHIPS}

2006 - 2009: Neuroscience Early Stage Research Training (NEUREST)

Fellow

2005 - 2006: Stipend International Max Planck Research School, Germany 2003 - 2005: Stipend Foundation for young scientists and artists, Ministry of Education, Government of Republic of Serbia

2000 - 2003: Stipend University of Belgrade 2001 - 2003: Stipend Smederevo City Government 


\section{List of publications}

Sumakovic M, Hegermann J, Luo L, HussonSJ, Schwarze K, Olendrowitz C, Schoofs L, Richmond J and Eimer S. UNC-108/RAB-2 and its effector RIC-19 are involved in dense core vesicle maturation in C. elegans, J Cell Biol in press

Marza E, Long T, Saiardi A, Sumakovic M, Eimer S, Hall DH and Lesa GM. Polyunsaturated fatty acids influence sinaptojanin localization to regulate synaptic vesicle recycling, Mol Biol Cell 2008 